\title{
IMPACT OF A MULTIMEDIA EDUCATIONAL TOOL \\ INCORPORATING THEORETICAL AND MIXED METHODS \\ ON THE FRUIT AND VEGETABLE INTAKES OF MIDDLE SCHOOL CHILDREN
}

\author{
JoAnne Louise Whelan
}

\author{
Submitted to the faculty of the University Graduate School \\ in partial fulfillment of the requirements \\ for the degree \\ Doctor of Philosophy \\ in the School of Health and Rehabilitation Sciences \\ Indiana University
}

May 2018 
Accepted by the Graduate Faculty, Indiana University, in partial fulfillment of the requirements for the degree of Doctor of Philosophy

Doctoral Committee

Brent Arnold, Ph.D., ATC, FNATA, Chair

Judith Ernst, DMSc, RD

Lisa Staten, Ph.D.

December 11, 2017

Paula Magee, Ph.D. 


\section{DEDICATION}

To my loving and supportive husband, Joseph, and my sons. 


\section{ACKNOWLEDGEMENTS}

Several individuals provided tremendous support to make this project a reality. This author wishes to sincerely thank Dr. Judith Ernst for her unwavering support and dedication to this dream. Her love of children and their health is truly admirable, empowering, and inspirational. Without her commitment, this project would not have come to fruition. This author also extends profound appreciation for the support of this research committee, Dr. Brent Arnold, Dr. Lisa Staten, and Dr. Paula Magee. Also, sincere, heartfelt thanks to Dr. Jessica Kanis, MD, Dr. Jacquelynn O’Palka, George Eckert, and the entire departmental staff within the School of Health and Rehabilitation Sciences at IUPUI for answering questions and navigating the complex academia world.

Special thanks to the entire Immaculate Heart of Mary Community, who allowed me to conduct this project as I pursue my life's work of educating and feeding children, and feeding them well. A special thank you to the physical education teacher, Mr. Kyle Burkholder for agreeing to this project and allowing access to his

classroom. Recognition is further extended to Laurie Trevino, for her knowledge and expertise in research semantics that were critical to the methodology design and implementation.

Finally, deepest gratitude is extended to my incredible husband Joseph Whelan, our sons, extended family, friends, classmates, and colleagues, who provided unconditional love and support throughout this process. Their guidance, laughter, and love were most needed and critical for the completion of this work. 
JoAnne Louise Whelan

IMPACT OF A MULTIMEDIA EDUCATIONAL TOOL INCORPORATING

THEORETICAL AND MIXED METHODS ON THE FRUIT AND VEGETABLE INTAKES OF MIDDLE SCHOOL CHILDREN

In the United States, over 30 percent of children are categorized as overweight or obese. Comorbid conditions, such as cardiovascular disease and other health complications related to obesity, are also on the rise. This public health issue is often related to disproportionate dietary intake and lack of physical activity. Efforts that promote fruits and vegetables $(\mathrm{F} / \mathrm{V})$ as preferred food choices over high fat and high sugar foods may help combat the increasing incidence of overweight and obesity. Other benefits from $\mathrm{F} / \mathrm{V}$ include prebiotics or fiber that helps to create and maintain a healthy microbiota, which is now recognized as essential for long-term positive health outcomes. Many children, however, fall short in consuming the recommended daily amounts of $\mathrm{F} / \mathrm{V}$ servings, and therefore, lack key nutrients such as vitamins, minerals, phytonutrients and fiber.

This study is a pilot, quasi-experimental design that provides information related to the importance of eating F/V to children, ages 11-12 years, who attend a parochial school in Indianapolis, Indiana. The data from this study describe the amounts of F/V servings in home-prepared school lunches. The primary aim of this study is to determine if eight interactive multimedia lessons and activities delivered to one group of students (intervention) and a single lesson delivered to a different group of students in the same school and grade (control), affects the amount of lunch F/V servings, student knowledge, attitude, and self-efficacy/perception. This baseline data will contribute to the design and 
implementation of a health curriculum for middle school age students. Focus groups, adapted validated assessment tools as well as the on-site observation of F/V servings brought in lunches and consumed at lunch are compared between both groups to document any effects of the instruction.

The results show that a statistically significant change in knowledge occurred within the intervention group following the implementation of this $\mathrm{F} / \mathrm{V}$ education series. Favorable findings, with a positive upward trend in relative amounts of $\mathrm{F} / \mathrm{V}$, were identified and more research in this area is warranted.

Brent Arnold, Ph.D., ATC, Chair 


\section{TABLE OF CONTENTS}

List of Tables............................................................... ix

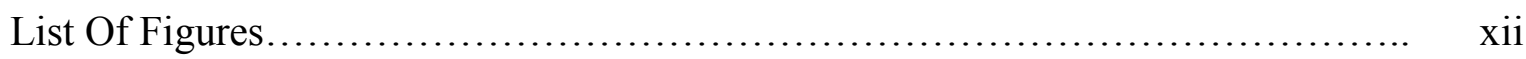

List Of Appendices.........................................................

Chapter I Introduction................................................ 1

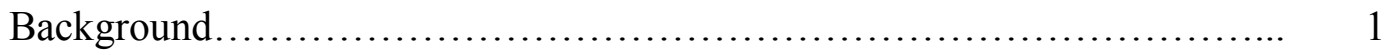

Statement Of The Problem................................................. 6

Overall Hypothesis............................................. 9

Study Aims........................................................ 14

Delimitations......................................................... 14

Assumptions...................................................... 15

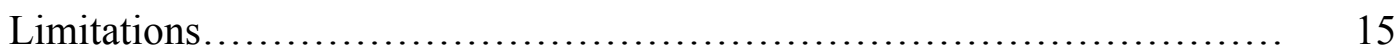

Significance Of The Study....................................... 15

Definition Of Terms..................................................... 17

Chapter II Review Of The Literature..................................... 18

Overview...................................................... 18

Prevalence...................................................... 26

Implications................................................. 28

Theoretical Framework: Education and Health Sciences.................. 32

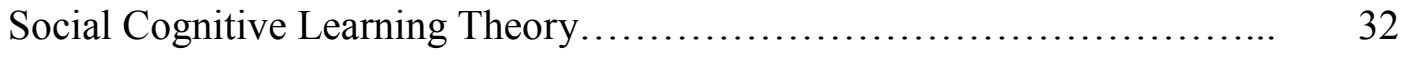

Self-Efficacy.................................................... 39

Health Belief Model............................................. 39

Chapter III Methods................................................... 54 
Intervention Overview

Data Collected....................................................... 63

Data Related to Study Aims......................................... 64

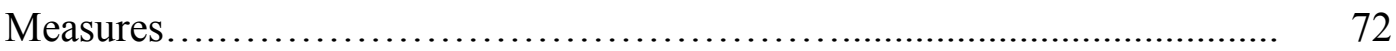

Chapter IV Results.................................................. 80

Chapter V Discussion................................................ 123

Chapter VI Conclusion................................................. 135

Chapter VII Appendices............................................... 142

Appendix A: School Lunch Federal Register Report..................... 142

Appendix B: Daily Reference Intakes (DRI) and Tolerable Upper Intakes..... 143

Appendix C: Approved protocol submitted to the Institutional Review Board. 145

Appendix D: Approved informed parental consent and child's assent forms... 157

Appendix E: Parent handouts given to students after the education lessons... 165

Appendix F: Focus group discussion probe questions for each group......... 171

Appendix G: Surveys/Forms.................................... 172

Appendix H: Scores for knowledge, attitude, self-efficacy/perception surveys 204

Appendix I: Statistics.......................................... 207

Appendix J: Raw data for intervention and control group participants......... 243

Chapter VIII References............................................ 303

Curriculum Vitae 


\section{LIST OF TABLES}

Table

1. Daily amounts of fruits and vegetables $(\mathrm{F} / \mathrm{V})$ recommended by the United States Department of Agriculture (USDA) for girls and boys.............. 4

2. The primary research hypothesis that includes the null and alternative counterparts.....................................................

3. Nutrients and components present in fruits and vegetables $(\mathrm{F} / \mathrm{V})$ that provide physiological benefits necessary for growth and development.

4. The content, justification and theoretical framework construct addressed for each of the lessons presented to middle-school children enrolled in an eightweek multimedia education program focused on the importance of fruit and vegetable intakes

5. Time frame for the study implementation activities and data collection with supported theory construct..........................................

6. Selected discussion probes and questions used in the focus groups to identify the Health Belief Model (HBM) constructs of perceived severity, perceived benefits, perceived barriers, cues to action, and self-efficacy as they relate to fruit and vegetable intake............................................

7. The variables measured in this research study and the complimentary statistical test used to assist with interpretation of the results.

8. Age, gender, and ethnicity of middle school children enrolled in an eightweek multimedia educational program focused on the importance of fruit and vegetable $(\mathrm{F} / \mathrm{V})$ intakes 
9. Identified themes for each construct of the Health Belief Model (HBM) and self-efficacy that emerged during focus group discussions conducted at baseline with middle school children enrolled in an eight-week multimedia educational program focused on the importance of fruit and vegetable (F/V) intakes.

10. The estimated number (\#) of fruit and vegetable (F/V) servings observed (baseline and post study) in home packed school lunches of middle school children enrolled in an eight-week multimedia educational program focused on the importance of $\mathrm{F} / \mathrm{V}$ intakes

11. Attitude and self-efficacy/perception scores derived from the Farm to Table assessment survey given to middle school children enrolled in an eight-week multimedia educational program focused on the importance of fruit and

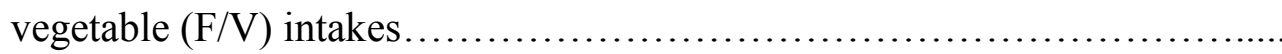

12. Percentages and relative amounts (mean \pm standard deviation) of fruit, potato, vegetable, and legumes using the 24-hour Farm to Table Survey at baseline and post study in middle school children included in an eight-week multimedia educational program focused on the importance of fruit and

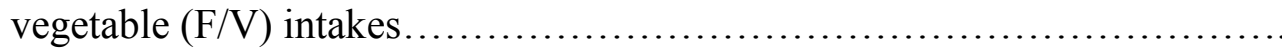

13. Number of fruit and vegetable $(\mathrm{F} / \mathrm{V})$ servings reported in the Day in the Life Questionnaire (DILQ) from breakfast through the after-school snack at baseline and post study and breakfast through after dinner snack at post study in middle school children enrolled in an eight-week multimedia educational program focused on the importance of $\mathrm{F} / \mathrm{V}$ intakes... 
14. Baseline and post study measures of a) height, weight, b) calculated body mass index (BMI) and BMI z-scores, and c) blood pressure (systolic and diastolic) of middle school children enrolled in an eight-week multimedia educational program focused on the importance of fruit and vegetable $(\mathrm{F} / \mathrm{V})$ intakes..........................................................

15. Comparison of the estimated number of fruit and vegetable (F/V) servings (mean \pm standard deviation) determined from all surveys at baseline and post study in middle school children enrolled in an eight-week multimedia educational program focused on the importance of fruit and vegetable (F/V) intakes........................................................... 


\section{LIST OF FIGURES}

Figure

1. Illustration of the Social Cognitive Learning Theory (SCLT) constructs and interrelationships with the behavior change process................ 33

2. The impact of the interconnectedness of self-efficacy and direct impact on self-regulation, along with social support, and outcome expectation on behaviors related to health behaviors

3. The Health Belief Model (HBM): The impact of the constructs of perceived susceptibility, perceived benefits, perceived barriers, self efficacy, cues to action, and perceived severity and the impact on readiness to act.......................................................

4. Schematic that shows the interrelationships of the Children's Health Belief Model (CHBM) based on demographics that impact the modifying factors, readiness factors, and behavioral factors

5. The proposed framework to improve fruit and vegetable $(\mathrm{F} / \mathrm{V})$ intakes in middle school children that combines Social Cognitive Learning Theory (SCLT), self-efficacy, and the Health Belief Model (HBM) in the program development for education sessions and focus group implementation

6. Schematic of data collection points including enrollment, baseline, intervention, and post intervention follow-up

7. The amount of fruit and vegetable (F/V) servings packed in school lunches compared to the estimated consumption at baseline and post 
study in middle school children included in an eight-week multimedia educational program focused on the importance of $\mathrm{F} / \mathrm{V}$ intakes...........

8. Pearson product correlation to determine the relationship between $\mathrm{F} / \mathrm{V}$ present in the meal and the consumption of $\mathrm{F} / \mathrm{V}$ in both groups...........

9. Knowledge scores (mean \pm standard error of the mean), at baseline and post study, obtained from the adapted Farm to Table survey in middle school children enrolled in an eight-week multimedia educational program focused on the importance of fruit and vegetable $(\mathrm{F} / \mathrm{V})$ intakes

10. Results of the Eating at America's Table Survey (EATS): Parent reported number of fruit and vegetable $(\mathrm{F} / \mathrm{V})$ servings offered to and consumed by their middle school children enrolled in an eight-week multimedia educational program focused on the importance of $\mathrm{F} / \mathrm{V}$ intakes

11. Results of the adapted Youth Adapted Food Frequency Questionnaire (YAQ): Parent weekly recalls of the number of servings within groups of fruits and vegetables $(\mathrm{F} / \mathrm{V})$ for their middle school children enrolled in an eight-week multimedia educational program focused on the importance of $\mathrm{F} / \mathrm{V}$ intakes.................................................

12. Results of the adapted Youth Adapted Food Frequency Questionnaire (YAQ): Parent monthly recalls of the number of servings within groups of fruits and vegetables $(\mathrm{F} / \mathrm{V})$ consumed by their middle school children enrolled in an eight-week multimedia educational program focused on 
the importance of $\mathrm{F} / \mathrm{V}$ intakes..

114 


\section{LIST OF APPENDICES}

Appendix

A. School Lunch Federal Register Report..................... 142

B. Daily Reference Intakes (DRI) and Tolerable Upper Intake

Levels. ...

C. Approved protocol submitted to the Institutional Review Board 145

D. Approved informed parental consent and child's assent forms...

157

E. Parent handouts given to students after the education lessons... 165

F. $\quad$ Focus group discussion probe questions for each group......... 171

G. Surveys/Forms.................................... 172

G-1. The adapted "Farm to Table" survey (Survey 1) questions 172

G-2. The "Day in the Life Questionnaire" (DILQ-Survey 2)...

181

G-3. The "Eating at Americas Table" (EATS-Survey 3)........ 185

G-4. The adapted "Youth-Adolescent Food Frequency

Questionnaire" (YAQ) weekly and monthly................... 194

G-5. Data collection forms used at baseline and post study..... 203

H. Scores for the knowledge, attitude, self-efficacy/perception

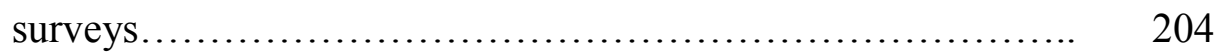

I. Statistics................................................... 207

Table I-1: Descriptive statistics from SPSS for each group including age, gender, height, weight, body mass index levels, z-scores, and blood pressure.

Table I-2: Intervention group statistical analysis with paired T- 
Tests for baseline and post study measures..................

Table I-3: Control group statistical analysis with paired T-Tests for baseline and post study measures.

Table I-4: ANOVA for personal data measures.

222

Table I-5: School lunch observations for the intervention and

the control groups

Table I-6: ANOVA for the "Farm to Table" survey (Survey 1)

including attitude, knowledge, self-efficacy/perception...........

Table I-7: ANOVA and descriptive statistics for the "Day in the Life Questionnaire" (DILQ-Survey 2) post study for all day dietary recall reported by the students

Table I-8: Mean and standard deviations for the adapted Eating at America's Table (EATS-Survey 3) parent report of fruit and vegetables $(\mathrm{F} / \mathrm{V})$ offered to and consumed by their child......... 228 Table I-9: Mean, standard deviations, and ANOVA for the adapted Youth-Adolescent Food Frequency Questionnaire

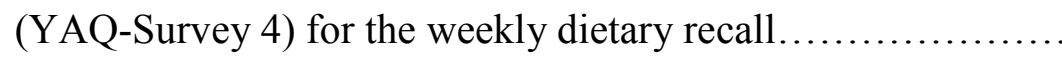

Table I-10: Mean, standard deviations, and ANOVA for the adapted Youth-Adolescent Food Frequency Questionnaire (YAQ-Survey 4) for the monthly dietary recall.................

Table I-11: Independent T-Test for Aim 2 baseline impact of gender, BMI, BMI Z-scores, blood pressure on the presence of fruits and vegetables in packed school lunch. 
Table I-12: Independent T-Test for Aim 2 post study impact of gender, BMI, BMI z-scores, blood pressure on the presence of fruits and vegetables in packed school lunch..................

Table I-13: Independent T-Test for parental involvement (grocery list completion) and post study observation and consumption for fruits and vegetables at school lunch..........

Table I-14: Correlation of observations and survey tools at baseline and post study.

J. Raw data for the intervention and control group participants... 


\section{CHAPTER I}

\section{INTRODUCTION}

\section{BACKGROUND}

Dietary recommendations for American children have changed over time as more connections are made linking nutrient consumption with health benefits. Specifically, with regards to fruits and vegetables $(\mathrm{F} / \mathrm{V})$, more servings are encouraged according to the Dietary Guidelines for Americans made available by the United States Department of Agriculture (USDA, 2012a). These guidelines reflect the federal nutrition policy standards for all individuals. Evidence suggests that despite these science-based recommendations, F/V intake remains well under the established levels (Krebs-Smith, 2001). Approximately 60 percent of Americans in general do not consume the daily recommendation of five servings of fruits and/or vegetables, $1 / 2$-cup servings each or approximately $2 \frac{1}{2}$ cups total (Casagrande, Wang, Anderson, \& Gary, 2007; Guenther, Dodd, Reedy, \& Krebs-Smith, 2006). More current information, from the Center for Disease Control (CDC), indicates that 90 percent of children (2-18 years) do not eat enough vegetable daily servings and 60 percent do not consume adequate amounts of fruit on a daily basis (CDC, 2014). As a result, many children's diets are lacking in crucial nutrients including potassium, phytonutrients, water, fiber, folate, ascorbic acid (Vitamin C), prebiotics and probiotics (Abuajah, Ogbonna, \& Osuji, 2015; L. Brown, Poudyal, \& Panchal, 2015; Dauchet, Amouyel, \& Dallongeville, 2009).

Children, birth to the age of 17 years, currently comprise 22 percent of the United States population according to the US Census Bureau. The percentage of those identified as obese or overweight is approximately 30 percent according to the CDC (CDC, 2008). 
World-wide, young people make up an estimated quarter of the population (United Nations \& Division., 2015) and this sub population is also the healthiest of all age groups (Das et al., 2017). In contrast, in adulthood (18-64 years of age), preventable diseases related to poor diet and other factors, are much higher (Gamble et al., 2017). Wellsupported research findings indicate many dietary habits are formed in the early life stages and are impacted by societal factors such as parenting and socioeconomics (R. Brown \& Ogden, 2004; Drewnowski \& Eichelsdoerfer, 2010; Emmett \& Jones, 2015).

Research over many decades supports the relationship between dietary consumption of $\mathrm{F} / \mathrm{V}$ and the reduction of diseases including diabetes, cardiovascular disease, cancer, and obesity (Bazzano, 2006). Over 2 million deaths worldwide are attributable to inadequate consumption of F/V yearly (Lock, Pomerleau, Causer, Altmann, \& McKee, 2005). Nutrition related health burdens, therefore impact public health as a result, as there are increased financial costs related to health care treatment in hospital settings for preventable diseases; an issue that is increasing exponentially in the United States, and across the globe (Bazzano, 2006; Steffen, 2006).

In 2005, the Institute of Medicine (IOM) and the Commission of the European Communities issued a report that addressed the worsening obesity epidemic, and chronic diseases associated with obesity (Steffen, 2006). This report highlighted the need for improvements in the affordability and availability of $\mathrm{F} / \mathrm{V}$ as a main prevention tactic to combat the rising rates of obesity (Steffen, 2006). Moreover, according to the IOM, the diets of children must be a main focus as dietary habits form in childhood, and this population should be at the forefront of public action due to the risk of developing chronic, treatable diseases in the earlier stages of life (McGuire, 2012) 
In accordance with goals developed by the IOM concerning children's health, clear messages to educate the population are necessary in order for the public to grasp and implement changes (McGuire, 2012). Current recommendations set forth from the US government health agencies separate the amount of $\mathrm{F} / \mathrm{V}$ by age groups and gender (CDC, 2014). Specifically, females ages $9-13$ years need $1 \frac{1 / 2}{2}$ cups of fruit and 2 cups of vegetables daily; while males require $1 \frac{1 / 2}{2}$ cups of fruit and $2 \frac{1}{2}$ cups of vegetables (Table 1) (CDC, 2014; USDA, 2010). 
Table 1: Daily amounts of fruits and vegetables $(\mathrm{F} / \mathrm{V})$ recommended by the United States Department of Agriculture (USDA) for girls and boys at different ages (USDA, 2010). 


\begin{tabular}{|c|c|c|}
\hline \multicolumn{3}{|c|}{ Girls } \\
\hline Age (years) & Fruit & Vegetables \\
\hline $2-3$ & 1 cup & 1 cup \\
\hline $4-8$ & $1-1 \frac{1}{2}$ cups & $11 / 2$ cups \\
\hline $9-13$ & $11 / 2$ cups & 2 cups \\
\hline $14-18$ & $1 \frac{1}{2}$ cups & $21 / 2$ cups \\
\hline \multicolumn{3}{|c|}{ Boys } \\
\hline Age (years) & Fruit & Vegetables \\
\hline $2-3$ & 1 cup & 1 cup \\
\hline $4-8$ & $1-1 \frac{1}{2}$ cups & $11 / 2$ cups \\
\hline $9-13$ & $11 / 2$ cups & $21 / 2$ cups \\
\hline $14-18$ & 2 cups & 3 cups \\
\hline
\end{tabular}




\section{STATEMENT OF THE PROBLEM}

As more young people, in early adolescence (10-14 years), have diets low in critical nutrients found in F/V servings, more health risk factors are likely to appear and result in chronic disease in adulthood (Delahanty et al., 2013). In 2009, approximately 30 percent more children were diagnosed with Type 2 diabetes compared to the start of the decade, with links to diet and activity changes as factors (Cree-Green, Triolo, \& Nadeau, 2013; Hamman et al., 2014). Disorders associated with cardiovascular disease and cancers are currently the main causes of death in adults and are related to poor diet and lack of weight control, and similar effects are apparent in the younger populations as well (Roger et al., 2011; Wang et al., 2014). The incidence of being overweight and obese in children in the United States (US) is 30 percent, which is considered high, and is often linked to inadequate F/V intake (Kim et al., 2014; Roger et al., 2011). Thus, more action is required to curb these trends.

Positive health outcomes including weight management and cardiovascular benefits from F/V intake are well documented (Jenkins et al., 2017; Lowry, Lee, McKenna, Galuska, \& Kann, 2008). The dietary components of F/V which include soluble and insoluble fiber (prebiotics), polyphenols, plant sterols, and probiotics are frequently identified as the nutrients that directly impact healthy outcomes (Abuajah et al., 2015). Consumption of the whole food is preferable to processed food products, to obtain the full benefit of these dietary components. Juices, for example, may have all fiber removed and sugar added; the prevalence of juice in the diet is often linked to undesirable weight gain (Boulton et al., 2016; Clemens, Drewnowski, Ferruzzi, Toner, \& Welland, 2015; Popkin \& Hawkes, 2016). 
Children, who are just entering the stage of adolescence, undergo a myriad of developmental processes that involve both physical and mental maturation. Energy needs in this population are often increased due to growth spurts and the onset of puberty (J. Brown, 2013). The flux in hormone levels may play a significant role because individuals enter puberty at varying times (Euling et al., 2008), and more nutrients are necessary to keep up with the demands of growth and development (Das et al., 2017).

This age group also may experience shifts in food intake that may not coincide with healthy habits recommended for long term benefits (van Jaarsveld, Fidler, Simon, \& Wardle, 2007) due to psychosocial changes that increase independence and decisions that include food choices (Scherf, Behrmann, \& Dahl, 2012). Focused instructions within the school environment are used as a strategy to help shape these decisions within family and social environments (Larson, Davey, Caspi, Kubik, \& Nanney, 2017). Gender has also been implicated as a factor in the frequency of $\mathrm{F} / \mathrm{V}$ servings in the diet as males have been identified as consuming fewer servings than females in a report published in the United Kingdom (Emmett \& Jones, 2015).

Given this background, several factors were considered in choosing the population to be studied and the environment for instruction. Narrowing the age range to 11-12 years allows for the observation of students in only one grade level, who are on the cusp of junior high school at some institutions, and seem by clinical observation to be pre-pubertal and pre-independent and, therefore, possibly more receptive to instructional interventions related to nutrition.

The school environment can have a significant impact on food and activity choices over periods of time and developmental stages (Mâsse \& de Niet, 2013; Morton, 
Atkin, Corder, Suhrcke, \& van Sluijs, 2016). In recent years, the United States federal government has mandated changes to school lunch as an effort to balance meals nutritionally and curb the threat of obesity in children. The 2012 USDA policy in conjunction with the Healthy Hunger-Free Kids Act, overhauled nutrition standards for meals served at public schools (USDA, 2012c). The standards include calorie goals for each meal based on the school level (elementary, middle, high school), more F/V servings; at lunch approximately 2 servings per day or $1 \frac{1 / 4}{4}$ cups for middle school students, and less fat and sodium (Appendix A).

Public elementary and secondary schools served an estimated 49.8 million American youth in the Fall of 2014 while private schools served an additional 5 million children according to the National Center for Education Statistics (NCES) (NCES, 2014); approximately 3 percent of school-aged children were homeschooled (NCES, 2013). Government policies regarding school lunch require adherence in public schools, however private schools and home environments are not required to adopt the same measures as schools that receive government funding (NCES, 2014). While fewer American children attend private schools or are homeschooled nationally, there are still many students yet to reach and educate about science-based nutrition for healthy living. Even though children in public schools are served $\mathrm{F} / \mathrm{V}$, data does not fully explain the degree to which these foods are selected and consumed. Therefore, there is a need for more information to ascertain the intake of $\mathrm{F} / \mathrm{V}$ food items in the school environment and methods that document and assess consumption. 


\section{OVERALL HYPOTHESIS}

The overall diet of students in early adolescence, and specifically between the ages of 11 and 12 years of age, frequently does not include the recommended amounts of F/V. Therefore, this age group is among those who are at increased health complications from obesity and other diseases that are linked to low $\mathrm{F} / \mathrm{V}$ intake. Changes to current food choices are therefore necessary and education directly suited for this age population is likely to be the first step to increase consumption of F/V. Nutrition education that includes interactive components within the school environment may be an effective strategy that will allow children to practice what they learn since they are "captive" and participating in this environment for a significant amount of time; on average 35 hours per week for 10 months out of the year, August through May.

The overarching question evaluated in this study, will determine if a multimedia interactive educational intervention that incorporates social cognitive learning theory, the health belief model, and self-efficacy will impact the amounts of F/V intakes from home prepared school lunches among middle school children, ages 11-12, as part of a convenience sample. This mixed method study rational is designed to gather exploratory information through qualitative data gathering with focus groups, to understand student viewpoints about $\mathrm{F} / \mathrm{V}$ intake (Creswell, 2008). The information and themes gathered will then influence the types of tools used in the intervention phase (i.e. curriculum, discussions, activities). Quantitative measures will then be employed to gather $\mathrm{F} / \mathrm{V}$ dietary intake changes through self-reports and direct observations. The primary hypothesis is that the amounts of F/V servings will increase in home prepared lunches of students, exposed to a multimedia interactive tool with constructs related to the social 
cognitive learning theory, health belief model, and self-efficacy (intervention group) compared to those with little or no exposure (control group). Those receiving the intervention will also demonstrate increases in attitude, knowledge, and selfefficacy/perception when compared to the control group. The primary research hypothesis that includes the null and alternative counterparts is presented in Table 2. 
Table 2: The primary research hypothesis that includes the null and alternative counterparts. 


\begin{tabular}{|c|c|}
\hline Null Hypothesis & Alternative Hypothesis \\
\hline Multi-media interactive tool & Multi-media interactive tool \\
\hline $\mathrm{H}_{0}: \mu_{\mathrm{A}}=\mu_{\mathrm{B}}$ & $\mathrm{H}_{1}: \mu_{\mathrm{A}} \neq \mu_{\mathrm{B}}$ \\
\hline $\begin{array}{l}\text { In the intervention group there is no } \\
\text { statistically significant baseline to post } \\
\text { study education difference in the amount of } \\
\text { F/V included in and consumed from home- } \\
\text { prepared lunches in the school environment; } \\
\text { attitude, knowledge, and self- } \\
\text { efficacy/perception compared to the control } \\
\text { group education. }\end{array}$ & $\begin{array}{l}\text { In the intervention group there is a } \\
\text { statistically significant baseline to post } \\
\text { study education difference in the amount } \\
\text { of F/V included in and consumed from } \\
\text { home-prepared lunches in the school } \\
\text { environment; attitude, knowledge, and } \\
\text { self-efficacy/perception compared to the } \\
\text { control group education. }\end{array}$ \\
\hline & $\begin{array}{l}\text { Directional Alternative Hypothesis } \\
\qquad \mathrm{H}_{1}: \mu_{\mathrm{A}}>\mu_{\mathrm{B}} \\
\text { The intervention group, with an eight- } \\
\text { lesson multimedia educational program, } \\
\text { demonstrates statistically significant } \\
\text { greater baseline to post education changes } \\
\text { in F/V servings included in and consumed } \\
\text { from home prepared lunches; attitude, } \\
\text { knowledge, and self-efficacy/perception in } \\
\text { the school environment compared to the } \\
\text { control group education. }\end{array}$ \\
\hline
\end{tabular}


A previous project completed by this research team found it feasible to engage a private parochial school in Indianapolis, Indiana and have parents and children agree to participate in a nutrition related study (Ernst \& Whelan, 2015). Results helped to determine the influence of personal factors on the $\mathrm{F} / \mathrm{V}$ intakes of children who attend a school that implements USDA federal guidelines for school lunch. Consumption of F/V during school lunch was captured with the following observations: (1) if at least one serving of either fruit or vegetable or F/V combined was present in the lunch; (2) if the intake of $\mathrm{F} / \mathrm{V}$ changed on different days of the week; and (3) if there was a relationship between home packed lunches, school prepared lunches, and the amount consumed at lunch time. The impact of individual characteristics gender, age, ethnicity, and body mass index (BMI) on F/V servings consumed at lunch was observed. No significant differences of F/V intakes were observed based on: (1) a child's individual characteristics (gender, age, ethnicity, or BMI), (2) where the lunch was prepared (home or school), or (3) the day of the week. Therefore, researchers concluded that a future study will be successful in a like environment and focused education about the benefits of $\mathrm{F} / \mathrm{V}$ in the diet can be provided to young people with the assumptions that gender, age, ethnicity, BMI, lunch source, and day of the week will not need to be considered as confounding variables. 


\section{STUDY AIMS}

Aim 1: To determine if an educational intervention, focused on the importance of $\mathrm{F} / \mathrm{V}$ in the diet and administered to middle school aged children (11-12 years) who attend a parochial Indianapolis-area school, impacts: (1) the amounts of F/V included in and consumed from home-prepared school lunches; (2) the students' attitudes toward F/V intake; (3) the students' knowledge of F/V benefits; and (4) and the students' selfefficacy/perception related to eating $\mathrm{F} / \mathrm{V}$.

Hypothesis: The impact of amultimedia eight lesson education program plus interactive feedback over eight weeks is significantly greater than the impact of one educational multimedia education lesson without interactive feedback, on the amounts and types of $\mathrm{F} / \mathrm{V}$ included in and consumed from home prepared school lunches, attitude towards $\mathrm{F} / \mathrm{V}$, knowledge of $\mathrm{F} / \mathrm{V}$, and self-efficacy/perception related to eating $\mathrm{F} / \mathrm{V}$ in middle school children ages 11-12 years.

Aim 2: To determine if personal factors such as BMI, blood pressure, and gender relate to the amounts and types of F/V included in and consumed from home-prepared school lunches among middle school children ages 11-12 years

Hypothesis: Factors at baseline, including BMI, blood pressure, and gender will not affect the amounts and types of F/V included in and consumed from home-prepared school lunches among school children ages 11-12 years.

\section{DELIMITATIONS}

This study is limited to participants from a convenience sample of students from a local, parochial, Indianapolis, Indiana-based school. Inclusion in this study met established age and developmental goals as advised by medical research and physician 
consultants. Students were assumed to have normal cognitive function if they were part of the normal classroom without modifications. Days for observation were limited to those when students brought home-prepared lunches; days were excluded when students purchased lunch provided by the schools.

\section{ASSUMPTIONS}

The assumptions made within this study include: (1) Participants are willing to be a part of this study even though consent was obtained by their parent; (2) Participants are provided with computers for normal school activities and are able to access educational modalities and surveys; (3) The outcome measures are generalizable to parochial schools and may not be suggestive of actions that may occur in public or home based schools; (4) The majority of students enrolled in the study have parents that are responsible for packing home prepared school lunches.

\section{LIMITATIONS}

This is a pilot study, and similar to other like studies of this scope, involves a small sample size. Most participants are from affluent backgrounds and are primarily Caucasian/white. Most participants are likely of higher learning abilities due to assumptions of the population.

\section{SIGNIFICANCE OF THE STUDY}

The educational modalities delivered in this study serve to educate this specific aged population by incorporating the health belief model constructs. The impact of the school environment on the health literacy of children is well supported in the literature (Bannon \& Schwartz, 2006). Various health agencies including the Academy of Nutrition and Dietetics, The Society for Nutrition Education, and American School Food 
Service Association support the need of health education to be present in schools at all grade levels, and that includes current information about recommended food intakes and the associated benefits (Position of ADA, 2003). Subsequently, Indiana state teaching standards for health and wellness have been identified and objectives are generalizable for nutrition health instruction. The following are the Indiana Department of Education Health and Wellness Standards for 2010 (DOE, 2010, pp. 40-42):

Standard 1: Students will comprehend concepts related to health promotion and disease prevention to enhance health.

The acquisition of basic health concepts and functional health knowledge provides a foundation for promotion health-enhancing behaviors among youth. This standard includes essential concepts that are based on established health behavior theories and models. Students apply knowledge of personal responsibility for health promotion and/or risk reduction. They describe patterns of healthy behaviors to prevent or reduce their risk of injury and/or illness throughout their lifespan. Students examine the interrelationships of emotional, physical, social and intellectual health and how they can be impacted by their surroundings.

(6.1.1--Compare how healthy behaviors and personal health are linked).

Standard 2: Students will analyze the influence of family, peers, culture, media, technology and other factors on health behaviors.

Health is impacted by a variety of positive and negative influences within society. This standard focuses on identifying and understanding the diverse internal and external factors that influence health practices and behaviors among youth including personal values, beliefs and perceived norms. Students compare how the family, peers, culture, media, and technology influence personal and family health. Students review how policies and regulations influence health promotion and risk reduction.

(6.2.1--Identify how family practices influence the health of adolescents). 


\section{DEFINITIONS OF TERMS}

1. F/V (FRUITS/VEGETABLES): This is a common abbreviation in the literature for fruits and vegetables. It is often used to describe servings or food items in these food categories in general (Neville et al., 2015).

2. OBESITY: A diagnostic condition that defines body mass index levels or the calculation of a person's body weight and body height that exceeds the healthy range level of normal for one's body frame. It is characterized by a BMI level for age and plotted on the CDC growth charts for children. The percentile score of 95 th $\%$ or above indicates obesity in children ages 2-20 years (CDC, 2016; Hurt \& McClave, 2016).

3. MICROBIOTA: Millions of different microorganisms adapted to survive and colonize in various sites in the human body, one being the flora of the gastrointestinal tract which is fundamental to the digestion and absorption of nutrients from food (Jeffery \& O'Toole, 2013).

4. PREBIOTICS: An indigestible fiber that serves to nourish probiotic components in the gastrointestinal tract (Principi \& Esposito, 2016).

5. PROBIOTICS: Microorganisms in the gastrointestinal tract that serve to fuel the cells in this organ of the body (Sanders, 2015).

6. HOME PREPARED LUNCH: Items that are bought and prepared by the home dwellers and sent to school for children to consume midday (Miles, Matthews, Brennan, \& Mitchell, 2010).

7. FUNCTIONAL FOODS: Specific foods in the diet, including fruits and vegetables, that show direct links to improved health outcomes (Ameratunga, Crooks, Simmons, \& Woon, 2016). 


\section{CHAPTER II \\ REVIEW OF THE LITERATURE}

\section{OVERVIEW}

Fruits and vegetables have been in the human diet for thousands of years. Some examples of the earliest $\mathrm{F} / \mathrm{V}$ include berries and wild plants as shown by several historical published articles (Crittenden \& Schnorr, 2017; Mukerji, 2006; Porter \& Marlowe, 2007). Anthropological evidence of dental decay is likely linked to the consumption of dates and legumes, in the diet of Neandertals (Cain, 2011).

Present day research supports the inclusion of $\mathrm{F} / \mathrm{V}$ in the daily diet. The Institute of Medicine (IOM) recognizes the necessity of these foods in the diet for many health benefits (IOM, 2001). Dietary References Intake (DRI's) include the Recommended Dietary Allowances (RDA), Adequate Intakes (AI) and Tolerable Upper Intake Levels (UL) for several nutrients found in F/V (Appendix B) (Cengage, 2004; DRI, 2006; Guenther et al., 2006; IOM, 2017). These nutrients include Vitamins A, B complex, C, E, K, and minerals such as potassium, calcium, magnesium, selenium, zinc, and iron. Other components in F/V with physiological health benefits include fiber, prebiotics, probiotics, and phytonutrients and are presented in Table 3 (J. Brown, 2013; Slavin, 2008; Solomons, 2006).

The importance of these dietary nutrients is significantly supported in the science field. Vitamin A is a fat soluble substance found in foods, is vital for eye sight, cell differentiation, gene regulation, immune function, reproduction, growth, membrane mucosa, skin, and bone health (J. Brown, 2013). Deficiencies are defined by increased risks of infections such as measles, and reduced eyesight, even potential blindness ( $\mathrm{J}$. 
Brown, 2013) and the inclusion of retinol improves gut homeostasis and immune function (Faria et al., 2013). Dietary forms of Vitamin A include carotenoids in F/V and retinol in animal products (Faria et al., 2013; Johnson \& Russell, 2010). Carotenoids, also known as provitamin A or precursors to vitamin A, are converted to retinol in the body and stored in the liver (Henning, Conaway, \& Lerner, 2015). Retinol, the most active form of vitamin A, is converted, as needed to less active forms, retinoic acid and retinal (Nagy, 2012). Lycopene and beta-carotene are the most abundant carotenoids and have the highest potential vitamin A activity (Beecher, 1998). The most common food sources of beta-carotene include carrots, broccoli, cantaloupe, and squash according to the IOM (IOM, 2001; Solomons, 2006). Significant impact on the immune system is noted with the inclusion of retinol or retinol precursors in the diet as they contribute to gut mucosal homeostasis; therefore, deficiencies have wide stemming impacts (Faria et al., 2013).

The water-soluble $\mathrm{B}$ vitamins available in $\mathrm{F} / \mathrm{V}$, include thiamin (B1), riboflavin (B2), pantothenic acid (B5), pyridoxine (B6), folate (B9), and biotin (H; B-complex vitamin). These substances serve to aid in digestion, energy metabolism, tissue repair, promote cellular growth, and immune function support (J. Brown, 2013). While deficiencies are rare, some consequences may include fatigue, weakness, impaired cellular growth, neurological disorders, and nausea/vomiting (J. Brown, 2013; CullumDugan \& Pawlak, 2015). Common dietary sources include green leafy vegetables, spinach, bell peppers, avocados, and citrus fruits (USDA, 2016b). 
Table 3: Nutrients and components present in fruits and vegetables $(\mathrm{F} / \mathrm{V})$ that provide physiological benefits necessary for growth and development. 


\begin{tabular}{|c|l|}
\hline Nutrient & \multicolumn{1}{|c|}{ Physiological Benefit* } \\
\hline Vitamin A & Immune function; eye sight \\
\hline B Vitamins & Digestion; metabolism; neurological function \\
\hline Vitamin C & Immune function; hormone synthesis \\
\hline Vitamin E & Antioxidant; gene formation \\
\hline Vitamin K & Blood clotting; bone formation \\
\hline Potassium & Acid-base balance; muscular function; metabolism \\
\hline Fiber & Gut function; elimination of waste, reduction of cholesterol \\
\hline Probiotics/Prebiotics & $\begin{array}{l}\text { Enterocyte benefit/stimulation of positive bacteria for } \\
\text { improved cellular function; aid digestion }\end{array}$ \\
\hline Calcium & $\begin{array}{l}\text { Bone and teeth development, nerve and muscle function, blood } \\
\text { clotting }\end{array}$ \\
\hline Iron & $\begin{array}{l}\text { Nerve and muscle function, bone and teeth formation, } \\
\text { metabolism }\end{array}$ \\
\hline Magnesium & Thyroid function, antioxidant \\
\hline Phytonutrients & Antioxidant; cardiac benefit \\
\hline Nerve and muscle function, enzyme production \\
\hline Transport oxygen, component of hemoglobin \\
\hline inc
\end{tabular}

*Brown, 2013; Slavin, 2008; Solomons, 2006 
Another water-soluble vitamin, Vitamin $\mathrm{C}$, is frequently found in $\mathrm{F} / \mathrm{V}$. The most common form, ascorbic acid, serves as an antioxidant to improve immune function, and is involved in the production of hormones and neurotransmitters (J. Brown, 2013; L. Brown et al., 2015). Signs of deficiencies include increased risk of infections, slower recovery from infections, decreased wound healing, fatigue, depression, and risk of bleeding or bruising (J. Brown, 2013). Food sources of ascorbic acid include citrus fruits such as oranges, lemons, and limes; and vegetables such as broccoli, peppers, tomatoes, asparagus, and collards (J. Brown, 2013; USDA, 2016b).

Vitamin $\mathrm{E}$ is a fat-soluble vitamin found in some vegetables. As an antioxidant, it aids in restored tissue damage, protection of cell membranes from oxidation, and gene expression (J. Brown, 2013). Signs of low levels of vitamin E include generalized weakness, hemolytic anemia, and nerve damage. Alpha-tocopherol is the form used in reference to vitamin E activity, and is present in leafy green vegetables and asparagus ( $\mathrm{J}$. Brown, 2013; USDA, 2016b).

Vegetables are also a source of fat-soluble, Vitamin K, phylloquinone or menaquinone. Vitamin K plays a role in blood clotting and bone formation (J. Brown, 2013). Bleeding, bruising, and low calcium levels may characterize deficiency of Vitamin K (J. Brown, 2013). Green vegetables and leafy vegetables are the most common sources of vitamin $\mathrm{K}$ in addition to grains (USDA, 2016b).

Potassium is a mineral found in $\mathrm{F} / \mathrm{V}$. It functions as an electrolyte, and promotes acid-base balance, heart function, cellular development, metabolism, and muscle function (Zwald, Elliott, Brownson, \& Skala, 2015). Common dietary sources of potassium include vegetables such as potatoes and fruit including bananas (USDA, 2016b). 
The most prevalent mineral in the body, calcium, is necessary for the development and maintenance of healthy bones and teeth (J. Brown, 2013; Martinez de Victoria, 2016). Dairy products and soft fish bones are considered the primary sources of dietary calcium, however some fruits, legumes, and green leafy vegetables are identified by individuals who follow a strict vegetarian diet as their sources of calcium; the amount available to the body, however is low because fiber and phytates that are also present in these plant sources inhibit calcium absorption (Martinez de Victoria, 2016).

Magnesium is a vital mineral for nerve function, bone and teeth formation, and metabolism (J. Brown, 2013). Food sources include F/V and legumes, especially chick peas (Costello, Wallace, \& Rosanoff, 2016). While consequences of magnesium deficiencies are rare, symptoms may include stunted growth in children, muscle weakness, and changes in behavior ((J. Brown, 2013; Costello et al., 2016). However, the National Health and Nutrition Examination Survey (NHANES) suggests dietary consumption of magnesium is often below recommended levels in the US, but the body compensates for this by decreasing levels of excretion primarily through the urine (Costello et al., 2016).

Zinc, selenium, and iron are also found in $\mathrm{F} / \mathrm{V}$, but in small amounts. Zinc is necessary for the production of enzymes for growth and development and is available in dried beans and other foods (J. Brown, 2013). A key antioxidant, selenium, is a mineral that is available in some vegetables, such as mushrooms, and is also critical for thyroid function (J. Brown, 2013; Olza et al., 2017). Iron, a transporter of oxygen and hemoglobin component, is critical for nerve and muscle function, prevention of fatigue, and more. Dried beans, dried fruits, and spinach are F/V sources that provide non-heme 
iron, a form that is less bioavailable than the heme iron sourced from animal products. The bioavailability of non-heme iron is improved when combined with food sources that provide vitamin C food sources (J. Brown, 2013)

Fiber is a non-digestible carbohydrate available in soluble and insoluble forms. The main role of insoluble fiber is to provide a plant-based bulky substance, which is used to aid in waste elimination (J. Brown, 2013). The benefit to the body is reduction of constipation and more regular stooling patterns. The edible skins and membranes of $\mathrm{F} / \mathrm{V}$ and legumes provide insoluble fiber. The fleshy parts of $\mathrm{F} / \mathrm{V}$ and legumes provide soluble fiber which creates a gel-like substance and promotes reduced rate of glucose absorption from other foods (MayoClinic, 2016). This is beneficial in many ways to prevent the risks associated with metabolic syndrome and diabetes; both which are linked to obesity (J. Brown, 2013). Food sources of insoluble fiber include whole-wheat breads, nuts, and beans. The F/V sources of soluble fiber include cauliflower, green beans, carrots, apples, citrus fruits and potatoes (MayoClinic, 2016).

Probiotics and prebiotics are at the forefront of research in terms of their impact on the gut microbiota. Probiotics are microorganisms that serve as fuel for enterocytes while prebiotics are non-digestible, plant-based fiber or carbohydrates, that serve as fuel for the probiotics (Principi \& Esposito, 2016). Two live microbials or probiotics, with beneficial effects to the host, Lactobacillus and Bifidobacterium, are often found in fermented foods such as yogurts, sauerkraut, and pickles (Markowiak \& Slizewska, 2017; Noverr \& Huffnagle, 2004; Papizadeh, Rohani, Nahrevanian, Javadi, \& Pourshafie, 2017). Prebiotics are fiber, often from F/V, which function as "food" to stimulate growth of good bacteria (probiotics), to create an optimal ecosystem in the gastrointestinal tract 
(Migliozzi, Thavarajah, Thavarajah, \& Smith, 2015; Tuohy, Fava, \& Viola, 2014).

According to Gibson and Roberfroid, the classification of prebiotics is determined if the fiber food source is not destroyed by gastric acidity or enzymes, and absorption occurs in the gastrointestinal tract; which allows for fermentation and growth of beneficial bacteria for the improvement of immune function and overall health (G. Gibson \& Roberfroid, 1995). Significant findings suggest that the addition of prebiotics in the diet have helped reduce the prevalence and duration of diarrhea associated with gastrointestinal upset, ailments related to inflammatory bowel disease and reduction in risks associated with colon cancer (Slavin, 2013). Prebiotics also include fructans, which are polymers of fructose in some $\mathrm{F} / \mathrm{V}$ that increase the bioavailability of bone enhancing minerals: calcium, magnesium and phosphorus thereby, enhancing the strength of bones and bone health (Abrams et al., 2005; Martin et al., 2010; Slavin, 2013). Cardiovascular health has also been improved with the reduction of low-density lipoproteins (LDL) when prebiotic fiber was included in the diet (Slavin, 2008, 2013). Finally, increased satiety due to the prebiotic composition of the diet has been reported along with weight loss and reduction of obesity (Hess, Birkett, Thomas, \& Slavin, 2011).

Phytonutrients, also called phytochemicals, are substances available in F/V. While not essential, research shows these compounds, such as: carotenoids, flavonoids, and phytoestrogens, play a role in the reduction of some disease conditions including cancer and heart disease (Corbi et al., 2016; Islam et al., 2016; Kristo, Klimis-Zacas, \& Sikalidis, 2016).

Foods that have been processed and fruit or vegetable juice versus whole fruits and vegetables remains debatable. While 100 percent $\mathrm{F} / \mathrm{V}$ juices fall into the food 
categories, limits are encouraged due to the omission of fiber in juices and the likelihood of excessive intake that will lead to an increase in natural sugar intake and likely subsequent weight gain beyond normal rates in children (Drewnowski \& Rehm, 2015; Pase, Grima, Cockerell, \& Pipingas, 2015). The American Academy of Pediatrics (AAP) recommends that children limit the amount of dietary 100 percent juice to four-six ounces per day, and to select whole foods more often (Drewnowski \& Rehm, 2015). Typically, children consume the greatest amounts of juices, compared to adults, and still fall short of the daily recommended F/V servings daily (Drewnowski \& Rehm, 2015).

\section{PREVALENCE}

Adapting a healthy diet in early childhood is critical for growth and development, as well as to prevent, future health risks (Hanson \& Gluckman, 2015; Helland, Bere, \& Overby, 2016). In the US, children and adults do not meet dietary recommendations for food servings, especially F/V (Helland et al., 2016). Health professionals recognize a lack of diverse food groups in the diets of children and factors associated with this are under investigation (Helland et al., 2016).

Childhood obesity is a significant public health issue in the United States (U.S.). While approximately a third of the population is obese, 17 percent of children and adolescents (2-19 years) were classified as obese in 2012 (CDC, 2008; DeNavas-Walt \& Protor, 2014; Taylor et al., 2016). Specifically, this problem is disproportionately affecting Latino children as approximately 22 percent (ages 2-19 years) in this minority group experience obesity versus 14 percent (ages 2-19 years) of Caucasian children (Ogden, Carroll, Kit, \& Flegal, 2014). This is likely related to excessive caloric intake, low physical activity, societal changes, and low F/V intake (Trost, Sirad, Dowda, 
Pfeiffer, \& Pate, 2003; Wijesinha-Bettoni, Orito, Lowik, McLean, \& Muehlhoff, 2013; Zhang \& Wang, 2008).

As noted, poor diet quality is often linked to higher levels of adiposity and is documented often among persons with lower socioeconomic levels (Jilcott Pitts et al., 2015). For example, children in lower socioeconomic households tend to consume more fruit juices or fruit drinks, which is associated with more weight gain (Drewnowski \& Rehm, 2015). Furthermore, low diet quality scores predicted by food frequency questionnaires (FFQ), indicate low intakes of $\mathrm{F} / \mathrm{V}$ and higher amounts of fat and sugar and correlated with higher BMI measures in children (Perry et al., 2015).

The parental impact on the diets of their children is also a significant factor (Martin-Biggers et al., 2015). Research supports that a child, who is classified as obese, is more likely to have a parent that is obese. While genetics plays a role, the environment is also a critical influence (Sylvetsky-Meni, Gillepsie, Hardy, \& Welsh, 2015) and food preferences of children are greatly influenced by parents in addition to dietary patterns and methods of preparation (Musher-Eizenman \& Kiefner, 2013). Therefore, it is essential that parents are educated and informed about necessary nutrients needed in the diet of children so that national food guidelines are met.

The Healthy People 2020 cites the importance and critical need to address the health concern related to childhood weight status. The current objectives are to achieve a 10 percent decrease in children and adolescents classified as obese by 2020 , which would reduce obesity rates for children ages 2 to 5 years from 10.7 percent to 9.6 percent; ages 6 to 10 years from 17.4 percent to 15.7 percent; and ages 12 to 19 years from 17.9 percent to 16.1 percent (USDHHS, 2016). 
The IOM recommends the use of multimedia campaigns as a strategy to increase awareness about childhood obesity (Koplan, Liverman, \& Kraak, 2005) and others acknowledge that there is as significant need for "innovative interventions" that address the obesity issues within the at risk adolescent population (Lubans et al., 2016).

\section{IMPLICATIONS}

Poor health often decreases quality of life, and increases the financial burden on individuals and society in general (Must et al., 1999). Nutrition status is often cited as a preventable contributor to chronic diseases (Salihu et al., 2016). Poor nutrition linked with poor overall health is a significant health disparity that often affects populations disproportionately, as lower income individuals face the higher risks of low quality dietary sources (Salihu et al., 2016). Approximately $\$ 379$ million is spent in the US to address nutrition specific programs that include food aid and increase food security (Ickes, Trichler, \& Parks, 2015). The Global Burden of Disease study suggests diseases directly related to the diet are on the rise (Lim et al., 2012). Recent reports also suggest,

In the USA, chronic diseases are the main cause of poor health, disability, and death, and account for most of health-care expenditures. Around half (50.9\%) of adults in the USA have at least one chronic condition and $26 \%$ have two or more conditions (Bauer, Briss, Goodman, \& Bowman, 2014, p. 45).

The consequences of obesity among the youth population can begin in childhood and adolescence and continue or manifest in adulthood as heart disease, diabetes and social stigmatizations (Biro et al., 2007; Flegal KM, 2010; Singh, Kogan, Van Dyck, \& Siahpush, 2008). Information in the academic literature supports the addition of dietary F/V to help reduce the risks associated with these conditions (Hansen, Alfonso, Hackney, \& Luque, 2015). 
Creating avenues for health behavior changes is at the forefront of health promotion investigations and research (Haberman, Brauer, Dwyer, \& Edwards, 2014). Fully gauging the change process may be the key to more successful health programs, especially in regards to $\mathrm{F} / \mathrm{V}$ intake as a way to reduce the prevalence of obesity (Annesi $\&$ Mareno, 2014). The Annesi group conducted research that tested the relationship between changes in self-efficacy, mood, and dietary self-regulation with F/V intake in obese adults. Increases in $\mathrm{F} / \mathrm{V}$ intake were found to correlate with improvements in the three factors studied and provided another approach to the management of obesity in this group (Annesi \& Mareno, 2014).

Gardening education programs have been used successfully as an approach to increase $\mathrm{F} / \mathrm{V}$ intake. A research group in California targeted Hispanic middle school aged children and conducted an interventional, education program over a four-month period (Somerville, Kessler, Wallace, \& Burns-Whitmore, 2012). Results indicated that the number of F/V servings, chosen as a snack, was doubled at the conclusion of this program (Somerville et al., 2012). A school based gardening program in Australia provided similar positive results and health promotion effects (Eckermann, Dawber, Yeatman, Quinsey, \& Morris, 2014).

A study conducted in Iowa involved the development of a nutrition program titled, "Nutri-Active Healthy Experience" (Matvienko, 2007). This pilot, quasiexperimental program was a four-week intervention for six and seven-year old children and, covered topics such as: energy balance, food composition, and health benefits of different food groups. The nutrition part of the program included a daily after-school snack as the intervention group was served snacks of high nutritional value (skim milk, 
yogurt, F/V, whole-grains, cheese, and 100\% juices) (Matvienko, 2007). The results showed that those in the intervention group where significantly more likely to choose $\mathrm{F} / \mathrm{V}$ snacks and other healthy choices when compared to those in the control group, who received no intervention (Matvienko, 2007).

Other nutrition education lessons formatted in a CD-ROM have also been developed. The "Nutrition Pathfinders" was created by the Dairy Council for students in grades third through fifth, and evaluated in California (Robertson \& Zalles, 2005). The study aim was to impact knowledge and attitudes toward healthy snack food selections. This interactive school-based nutrition education program successfully influenced student knowledge and attitudes toward nutrition (Robertson \& Zalles, 2005).

The "5 a day game" was used in a study by Rosi and associates to educate children ages 8-10 years about healthy food choices, over a three-month period, using video games and lessons labeled "edutainment" in Italy (Rosi et al., 2015). The results from this study showed significant increases in both $F / V$ intakes by almost 67 grams per day, which equates to about one-half serving per day, and the researchers concluded that knowledge about the nutritional benefits of $\mathrm{F} / \mathrm{V}$ "appears to be a paramount factor" to increase intake and improve diet quality (Rosi et al., 2015). European recommendations are the same as those in the US, approximately 5 portions of F/V daily, which is equivalent to approximately 400 grams per day (Evans, Christian, Cleghorn, Greenwood, $\&$ Cade, 2012). Therefore, the change noted in the " 5 a day game" was approximately a 16 percent increase in $\mathrm{F} / \mathrm{V}$ intake.

The Bannon research group studied the effects of showing kindergarten children, from a school in Connecticut, one of three different types of sixty-second educational 
videos about F/V (Bannon \& Schwartz, 2006). Each of the three subject groups only viewed one video, one time, have either a gain framed video, a loss framed video, or a control video. After the video, the study participants were given the choice of apples or animal crackers for a snack. The groups that watched the gain $(\mathrm{p}=0.059)$ or loss $(\mathrm{p}<$ 0.005) framed videos more often chose the apples as a snack compared to the control group. The researchers noted that only one exposure to the videos was possibly not enough to convey the information, and perhaps repeated videos may have had more significant impacts (Bannon \& Schwartz, 2006).

School-based interventions were reviewed in a meta-analysis of trials to help educate students about the importance of F/V intake (Evans et al., 2012). These researchers reviewed Ovid, Cochrane, PsycINFO, EMBASE, and the Global Health library databases to summarize data from studies that involved children ages 5-12 years that also included dietary recall assessment tools (Evans et al., 2012). The findings show that any school-based intervention improved daily $\mathrm{F} / \mathrm{V}$ consumption by an average of one-quarter to one-third of a portion per day; equivalent to a 20-30-g daily increase per day (Evans et al., 2012). However, there is no information that supports the longevity and sustainability of this change. In addition, national statistics continue to reflect an overall marginal intake of $\mathrm{F} / \mathrm{V}$, suggesting possibly not enough schools address dietary guidelines or possibly a new approach, based on health theories is necessary and needed for long term improvements in $\mathrm{F} / \mathrm{V}$ selection and consumption. 


\section{THEORETICAL FRAMEWORK: EDUCATION AND HEALTH SCIENCES}

The focus on types of theories, within education and health and rehabilitation sciences, are at the forefront of research in these fields. Theory in general begins with an ontological and epistemological background that is derived from a multitude of observations that then is translated into the behaviors humans enact, such as health behaviors (Durham, Sykes, Piper, \& Stokes, 2015). In this study, specific theories are used as a framework that merge the educational and behavioral health sciences aspects of curriculum design and presentation to middle school age children. These theories include the social cognitive learning theory (SCLT), self-efficacy, and the health belief model (HBM). It is believed these three approaches blend interpersonal and intrapersonal constructs to most effectively cause behavior change.

\section{SOCIAL COGNITIVE LEARNING THEORY}

The interpersonal SCLT is illustrated in Figure 1, and is often used in educational behavioral studies (Bandura, 1994). The SCLT constructs are interrelated and impact the behavior change process as a whole (Tadayon Nabavi, 2012). This theory assumes that behavior is influenced by both environmental and personal factors; these factors impact each other, and both impact behavior. It is also assumed that knowledge is gained by observing others, and one is then able to change behavior or achieve established health related goals (Hayden, 2009). The construct of self-efficacy was later added to this model to encompass how personal thoughts and judgments may impact health behaviors (Ashford \& Lecroy, 2010). 
Figure 1: Illustration of the social cognitive learning theory (SCLT) constructs and interrelationships with the behavior change process (Tadayon Nabavi, 2012). 
Behaviour

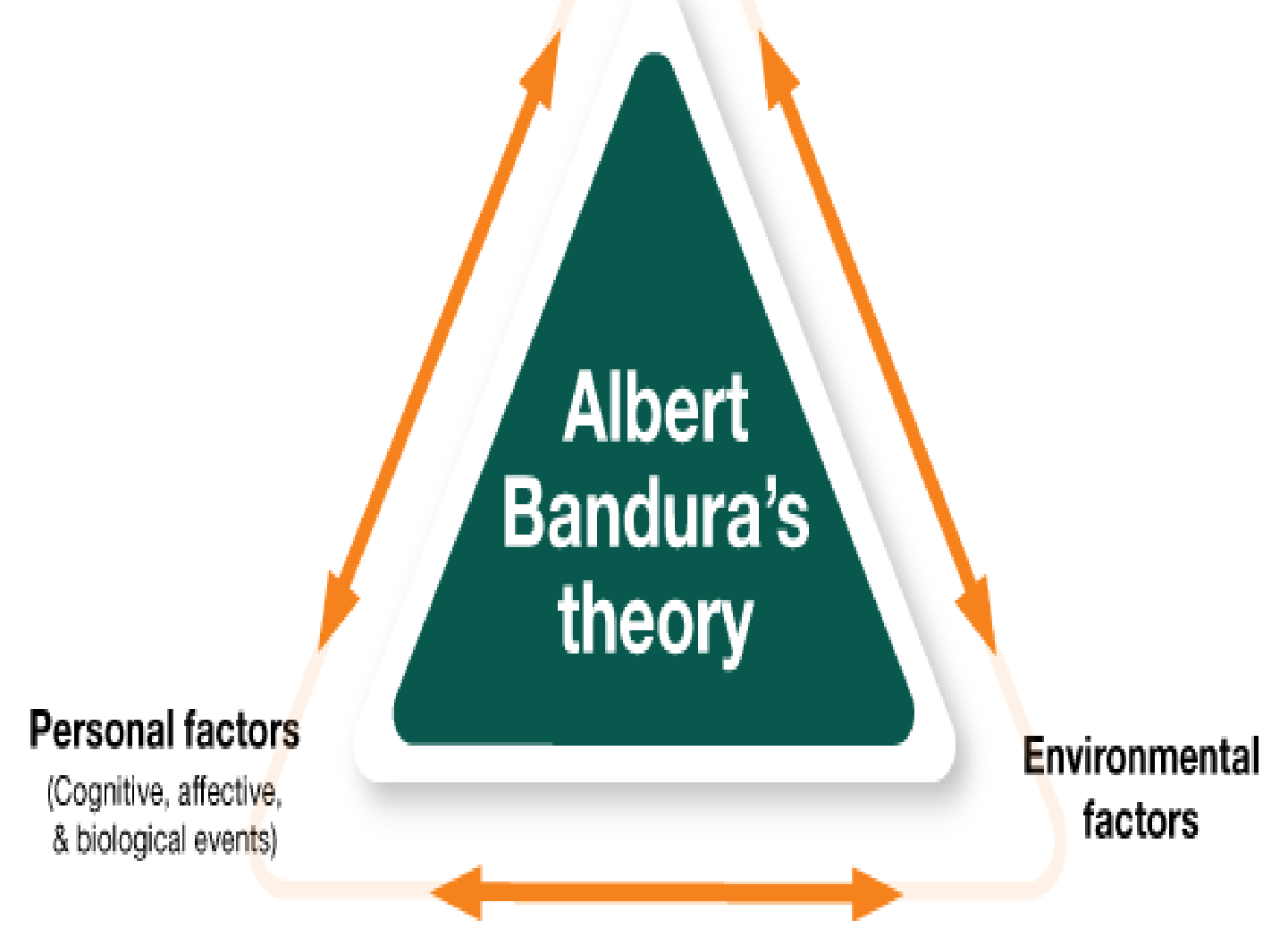


Several studies have assessed the use of SCLT in nutrition education. Most research involves the relationship between dietary behavioral changes and cardiovascular disease, diabetes, and weight management; and unsurprisingly, these are the highest disease risks in the US (Burke, Dunbar-Jacob, Orchard, \& Sereika, 2005; Glasgow, Toobert, Mitchell, Donnelly, \& Calder, 1989). The nutrition workgroup, led by Spahn and team, reviewed several articles related to behavior change theories and concluded that SCLT is the most commonly used theory in group settings, and recommends that more research needs to be done. (Spahn et al., 2010).

Several experts suggest incorporating the SCLT in interventions focused on nutrition education for adolescents and their approach to healthy eating. The Dewar group commented that health behavior interventions guided by relevant theory (e.g. Social Cognitive Theory, Self-Determination Theory) are more effective in changing behavior versus non-theoretical approaches. These theories postulate that an intervention's effects are achieved through constructs such as self-efficacy or perceived benefits, that operate in ways to enable a path between an intervention and behavior change (Dewar, Lubans, Plotnikoff, \& Morgan, 2012). Results from school based weight management programming for adolescents, based on the constructs of knowledge and self-efficacy, indicate a correlation between more healthful dietary choices with greater levels of confidence (Hall, Chai, Koszewski, \& Albrecht, 2015). Therefore, there is impactful evidence that suggests the usefulness of behavioral theories in research and the benefits in practice will likely be substantial in this population.

The SCLT is also used successfully in interventions with children to address interpersonal factors that influence actions including personal, environmental and 
behavioral constructs (Hayden, 2009; Nixon et al., 2012). The Nixon group reviewed studies that employed a theoretical framework that specifically addressed physical and/or dietary changes that would impact weight status in young children (Nixon et al., 2012). Of the 12 studies included in the assessment, most included SCLT as the framework for their weight management program. The programs that had the most significant results, including improved weight management, dietary changes with increased F/V intake, physical activity improvement, or parental involvement, most frequently incorporated the SCLT theory (Nixon et al., 2012). The role of parental involvement at medium or high levels was also of importance as parents serve a significant role in the overall development of the child (Nixon et al., 2012).

In 2011, the Anderson-Bill group looked at how SCLT in web based health education programs impacted individuals and their health behavior changes. The authors clarified that their study examined the psychosocial, behavioral, and demographic characteristics of internet-health users recruited for a web based social cognitive theory (SCT) program that included nutrition information, physical activity, and weight gain prevention (Anderson, Winett, \& Wojcik, 2007). The researchers concluded that successful treatment plans should use SCT in the education process and that the degree of success for those that are web-based interventions focused on physical activity and nutrition education depends on the extent to which participants are encouraged to develop self-efficacy for behavior change. Success also depends on social support for making changes, setting achievable goals, planning, tracking, and receiving feedback for specific behaviors as related to self-efficacy, self-regulation, health behavior and the expected outcome as presented in Figure 2 (Anderson et al., 2007). 
Figure 2: The impact of the interconnectedness of self-efficacy and direct impact on selfregulation, along with social support, and outcome expectation on behaviors related to health behaviors (Anderson-Bill, Winett, \& Wojcik, 2011). 


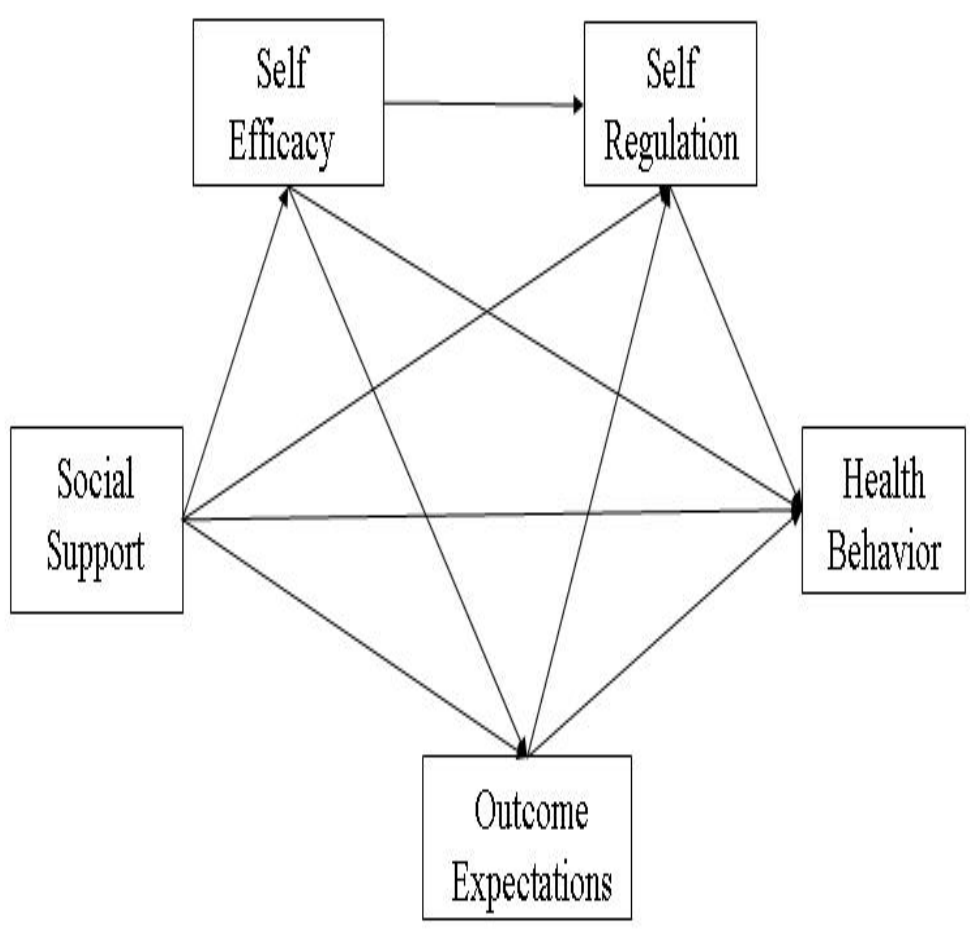




\section{SELF-EFFICACY}

In education and health science, the impact of the self-efficacy theory, on its own, is apparent. For example, if the level of self-confidence or personal judgments related to a goal remains low, the desired outcome may not be achieved (Fugl-Meyer, Hellström, Lindmark, \& Wahlberg, 2003). A few studies have assessed self-efficacy related to F/V intakes in children to determine the validity of the nutrient intake assessment tools, and/or the relationship between F/V intakes measured by food frequency questionnaires (FFQ), self-efficacy and behavior (Baranowski et al., 2010; Watson et al., 2006). Authors concluded that more studies would be helpful to determine predictive behavioral patterns in this age grouping.

The SCLT together with self-efficacy is often used with other theories possibly suggesting it may be best utilized in conjunction with other constructs in different health theories (Nixon et al., 2012). Self-efficacy is believed to serve as a bridge or commonality in health theories that may encapsulate more concrete descriptions and predications related to behavior changes (Geller, Dzewaltowski, Rosenkranz, \& Karteroliotis, 2009). An expansion of the theory of self-efficacy, HBM, ads individual perceptions and modifying factors that are believed to impact the likelihood of the action related to the behavior change (Hayden, 2009).

\section{THE HEALTH BELIEF MODEL}

The HBM is a psychosocial approach to understanding perceived susceptibility, perceived seriousness, perceived benefit, perceived barriers, cues to action, and selfefficacy in relation to health (Figure 3) (Hayden, 2009). The HBM, developed in the 1950’s by social psychologists Irwin M. Rosenstock, Godfrey M. Hochbaum, S. Stephen 
Kegeles, and Howard Leventhal (HBM, n.d.; Rosenstock, Strecher, \& Becker, 1988), and later restructured to include additional constructs by Janz and Becker (Janz \& Becker, 1984), has been used in nutrition studies to evaluate nutrition knowledge and more (Iranagh, Rahman, \& Motalebi, 2016; Jeihooni, Hidarnia, Kaveh, Hajizadeh, \& Askari, 2016; H. S. Kim, Ahn, \& No, 2012). The HBM often aligns with supportive explanations and insight into why health behaviors are or are not regularly adapted based on the impact of confidence or benefits/barriers (Conn, Enriquez, Ruppar, \& Chan, 2016; Kronish, Leventhal, \& Horowitz, 2012; Mennella \& Beauchamp, 2008). The HBM is therefore, one of the most widely used intrapersonal health theories used in health education (Glanz, Rimer, \& Viswanath, 2016) and is frequently the framework used for testing educational interventions in the research field (Janz \& Becker, 1984; Rezapour, Mostafavi, \& Khalkhali, 2016; Yang et al., 2016).

Several nutrition education studies have used the HBM with children. The Nixon group highlighted studies with the most effective education incorporated behavioral theories/models for reducing the rates of childhood obesity, as well as medium to high levels of parental involvement (Nixon et al., 2012). Collectively, these results provided evidence that theoretical models are essential in research, in order to have the most beneficial outcomes related to nutrition behavior changes in children, such as improved weight status and dietary choices (Nixon et al., 2012). 
Figure 3: The health belief model (HBM): The impact of the constructs of perceived susceptibility, perceived benefits, perceived barriers, self-efficacy, cues to action, and perceived severity and the impact on readiness to act (Eng, 2014). 


\section{Health Belief Model (HBM)}

(Hochbaum, Rosenstock \& Kegels: 1950s)

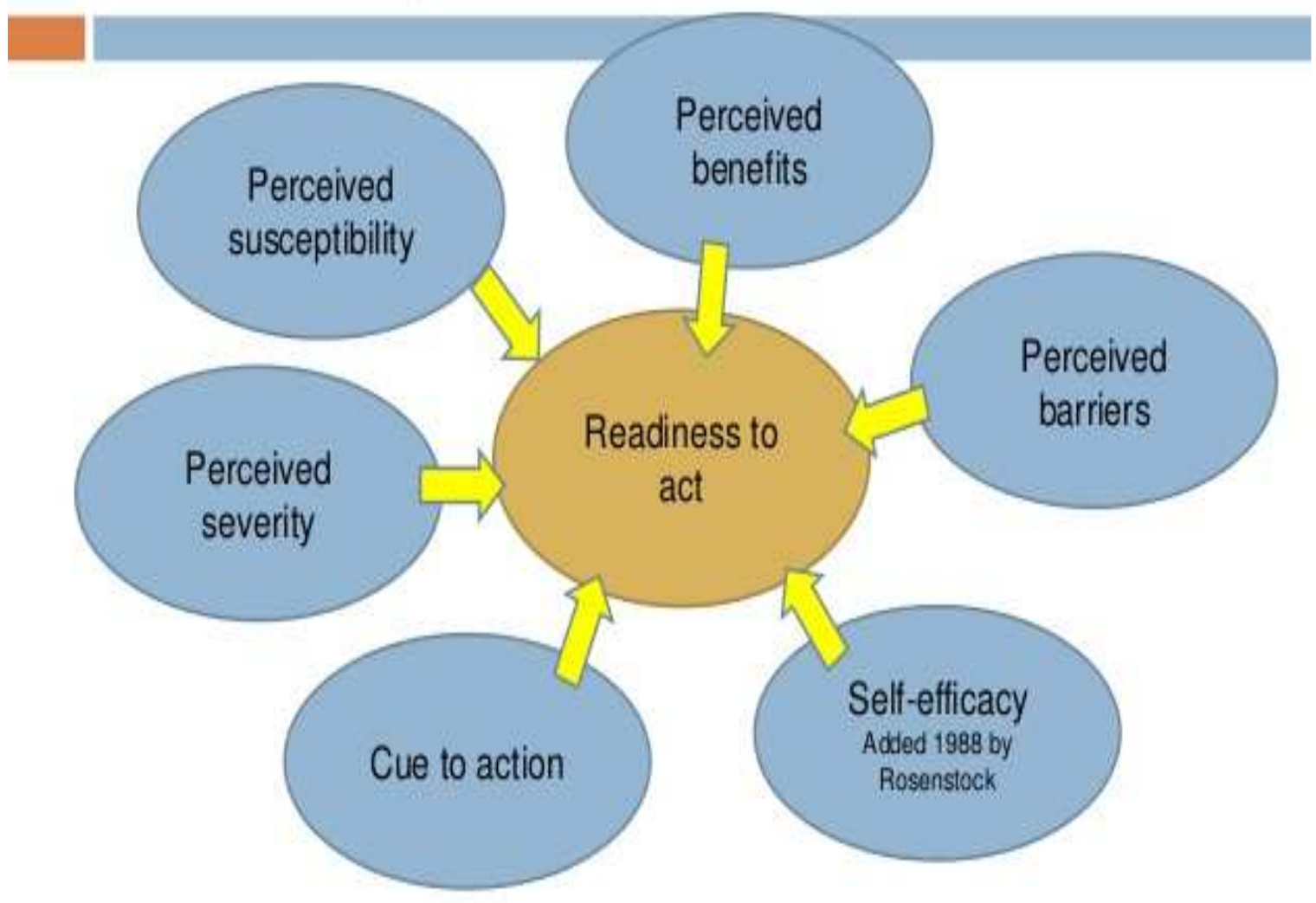


A study with adolescents in Nebraska instituted HBM based nutrition education sessions focused on folate intake. The results did not show an increase in folate intake from baseline to post study, possibly due to omission of parental involvement in the design of the study. Daily estimates of folate intake at both time points, however, were equal to the RDA of $400 \mathrm{mcg}$ (LaBrosse \& Albrecht, 2013). Knowledge scores did significantly change from baseline to post intervention, which supported that the information presented was learned (LaBrosse \& Albrecht, 2013).

A pilot study, conducted in Beirut school aged children used the HBM framework to evaluate the education component related to beverage choices (Abi Haidar, Lahham Salameh, \& Afifi, 2011). Nutrition lessons were delivered over 50-minutes on four occasions to a group of third and fourth grade students $(\mathrm{n}=110)$. Knowledge, attitude, self-efficacy, and intake of water were measured pre- and post-intervention. The results indicated that post intervention scores were significantly higher in the knowledge category ( $\mathrm{p}=0.000)$. Compared to the baseline intervention, students at the end of the study felt more confident to drink fewer soft drinks and more water $(\mathrm{p}<0.05)$, to drink water if they felt thirsty $(\mathrm{p}<0.05)$, and to select water over soft drinks when eating in a restaurant $(\mathrm{p}<0.05)$. The percentage of students drinking six or more cups of water increased by approximately 30 percent $(\mathrm{p}=0.000)$; and those drinking less than one can of soft drink/day increased by approximately 32 percent $(p=0.000)$ (Abi Haidar et al., 2011).

The Burnet group proposed a model for Type 2 Diabetes management among minority populations that would encompass theories including the SCLT and HBM with emphasis on self-efficacy (Burnet, Plaut, Courtney, \& Chin, 2002). This team reviewed 
data completed in the 1980's through the early 2000's to assess the best intervention approach to change dietary related behaviors associated with managing Type 2 Diabetes (Brownell, Kelman, \& Stunkard, 1983; Epstein, Paluch, Gordy, \& Dorn, 2000; Kelder, Perry, Lytle, \& Klepp, 1995). The population reviewed included youth and knowledge and self-efficacy changes were measured. The researchers concluded that interventions fail when they are not based on behavior change theories, and they recommended an approach that includes theory/models that address all aspects of human influence on behavior adaptations related to management of Type 2 Diabetes (Burnet et al., 2002).

A randomized controlled study in Australia that addressed obesity prevention was conducted at multiple sites with five to nine year old children ( $\mathrm{n}=165)$ (Burrows, Warren, Baur, \& Collins, 2008). The study was designed to gain an understanding of the dietary intakes and behavior changes of overweight and obese children in a program that incorporated constructs of the HBM for approximately one year. Three separate intervention groups were included: (1) a parent-centered nutrition lifestyle program; (2) a child-centered physical activity skill development program; or (3) both of the programs. A food frequency questionnaire (FFQ) was used in all groups to estimate nutrient intake at baseline and repeated at 6 months, and 12 months after the intervention to determine changes. All groups improved nutrient intakes with no differences detected between groups ( $p>0.05)$. Total quantity of food decreased significantly at both time points $(\mathrm{p}<0.05)$. The percent energy derived from core food groups, minus fruit, increased significantly at 12 months compared to baseline $(\mathrm{p}<0.05)$, and non-core foods decreased, with the largest decreases in the sweetened drinks category $(\mathrm{p}<0.001)$ and packaged lunch box snacks $(\mathrm{p}<0.001)$. The intervention programs in all of the treatment groups 
appeared to be equally effective in improving the dietary intake of overweight and obese children (Burrows et al., 2008).

A study conducted in Pennsylvania, incorporated the HBM in a nutrition education program for fourth to eighth graders $(n=1619)$ (Cheng, Monnat, \& Lohse, 2015). Over two years, students participated in one of three groups: (1) cooking and tasting curriculum (2) tasting curriculum, or (3) control with no intervention. A curriculum based on the HBM constructs and focused on the importance of calcium in the diet was incorporated into the regular classroom, with the goal to increase dietary intakes of calcium containing foods by hosting food demonstrations and taste tests. The results indicated for students who did enjoy the food tasting, over 60 percent, were more interested in the lessons compared to those students who did not enjoy the food tasting ( $p$ $<0.001)$ (Cheng, Monnat, \& Lohse, 2015).

A nutrition pilot study involving mothers and children in a Somali Community in Minnesota was completed in 2016 (Kehm, Hearst, Sherman, \& Elwell, 2016). The intervention was designed specifically for low-income mothers and children $(\mathrm{n}=25)$. The program incorporated constructs of the HBM and self-efficacy as an attempt to address the mothers' ability to serve more F/V servings to their children; improve knowledge and beliefs related to healthy dietary intakes; and highlight perceived barriers for obtaining healthy foods. The results, from baseline to post study, showed that self-efficacy, measured with a seven-item survey specific to the study, significantly increased among participants $(\mathrm{p}=0.01)$. No significant changes in knowledge, beliefs, or perceived barriers were found from the start compared to the end of the intervention, however, daily servings of $\mathrm{F} / \mathrm{V}$ (measured by survey report completed by the mothers) were significantly 
increased among women and children $(\mathrm{p}=0.01$ to $\mathrm{p}<0.01)(\mathrm{Kehm}$ et al., 2016).

A similar study with both mothers and children was conducted in Ethiopia (Mulualem, Henry, Berhanu, \& Whiting, 2016). The goal of this study was to measure the impact of nutrition education, using the HBM, to promote the inclusion of legumes in foods for infants and toddlers that complement breastfeeding. At baseline, mid, and completion of the study, knowledge, attitude and dietary practices questions were given to the mothers in the intervention groups (education that included recipes demonstrations twice per month for 6 months) and compared to the control group (usual education). At baseline, the mothers' knowledge, attitude, and dietary practice scores were low in both the intervention and control groups. At the midway point and completion of the study, the intervention group had significantly increased knowledge, attitude, and dietary practice scores $(p<0.05)$ compared to the control group. The differences were reflected in the post study nutritional status of the children with improvements in mean weight, weight for height, and weight for age in the intervention group only (Mulualem et al., 2016).

A child-centered version of the HBM: The Children's Health Belief Model (CHBM) was introduced in 1990 (Bush \& Iannotti, 1990) and is presented in Figure 4. The goal of this model was to further consider the stages of child development as behaviors are assessed. The impact of family and peers on decisions made with regards to health behaviors was also considered. While data supported the use of this model, it is not widely available in the literature. 
Figure 4: Schematic that shows the interrelationships of the Children's Health Belief Model (CHBM) based on demographics that impact the modifying factors, readiness factors, and behavioral factors (Bush \& Iannotti, 1990). 


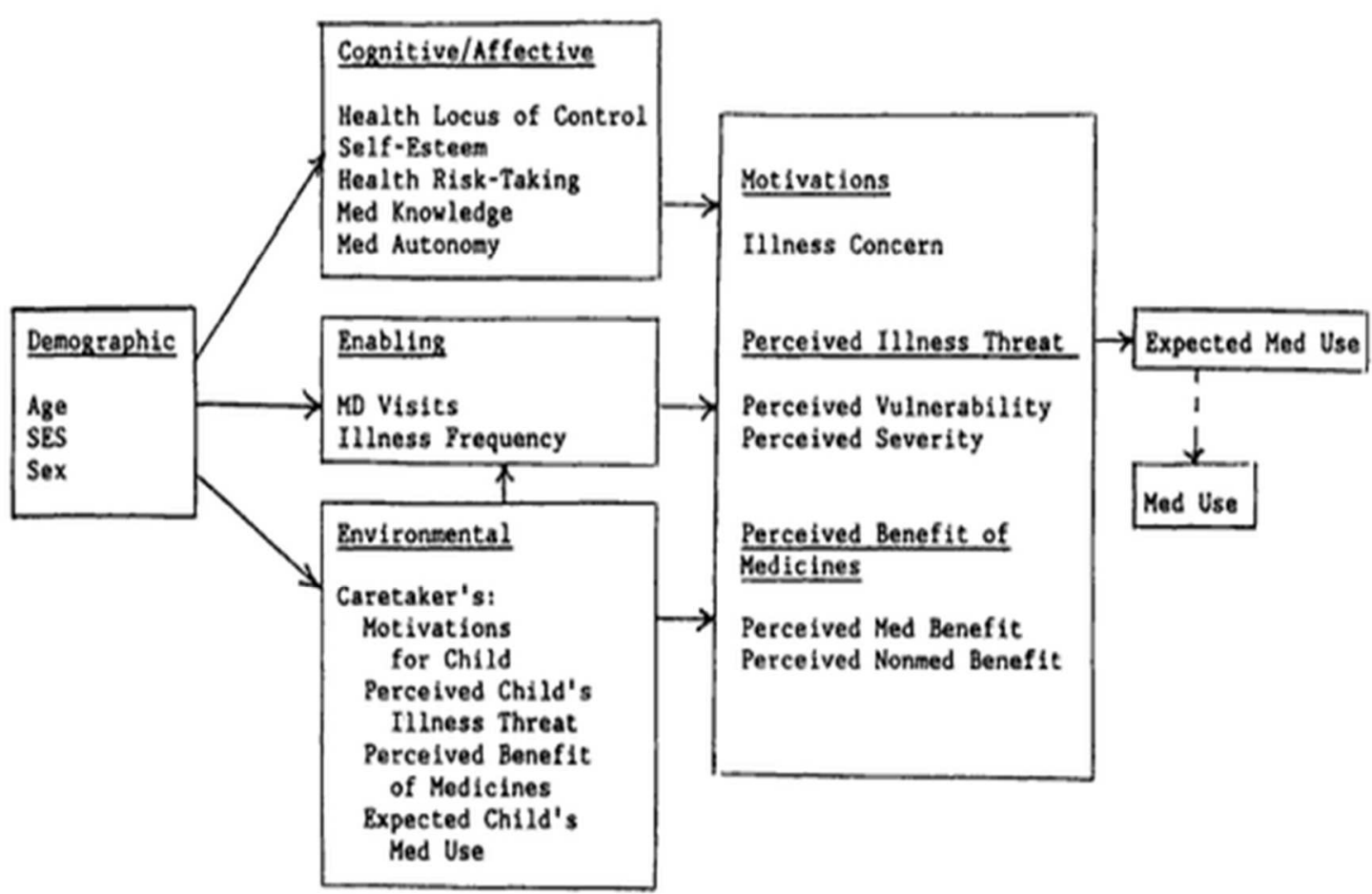


Fenton has suggested an adolescent health belief model (AHBM) to assess the barriers of medication administration in this age population that includes the addition of two constructs to the traditional HBM; peer support and perceived control (Fenton, 2014). While they appear to be reasonable additions in the design, no other studies have been located to further support these additions in the literature, especially related to nutrient intake.

Study designs, already discussed, support the use of the HBM; results of these studies demonstrate the strong influence that parents have in the provision of food to their children and identify the multiple dimensions that exist surrounding the social determinants of F/V intake. An analysis of how the parent-child dyad reports compare with each other may provide valuable insight into food choices and the development of educational content that is focused to increase daily F/V intakes for children at home and at school.

An expanded theoretical framework is used in the design of the pilot study presented in this thesis and incorporates the HBM, as well as self-efficacy; the HBM emphasizes the interrelationships of the environment, interpersonal factors, and health behaviors and self-efficacy enables the full exploration of the comfort that one has with a health behavior so that it can be initiated, and with practice, becomes routine (Bandura, 1994; Luszczynska, 2005).

The HBM is often cited as the framework used in a myriad of qualitative studies that use focus groups to gather data. Some examples include studies that evaluate oral health in children and have used the HBM to understand viewpoints of this population (Bonvecchio et al., 2014; Walker \& Jackson, 2015). Other researchers have used the 
HBM theoretical approach to understand how to clinically improve medication adherence in children with mental health issues thereby enhancing the relationship between parents, practitioners, and patients (Charach, Volpe, Boydell, \& Gearing, 2008). Researchers who wanted to understand reasons why parents seek respiratory treatment for their children in the United Kingdom noted the level of confidence parents have after receiving consistent, trusted information from practitioners (Ingram, Cabral, Hay, Lucas, \& Horwood, 2013).

More specific to nutrition, the HBM served as the framework in several qualitative studies involving parents and children. The Troxell group wanted to develop education materials that would be used in WIC programming to teach mothers about the benefits of omega 3 fatty acids in their diet for fetal development (Troxell et al., 2005). According to researchers, the HBM helped address the needs of the participants, barriers, and also motivators to making the dietary adjustments (Troxell et al., 2005). Another study that addressed food safety in families was conducted to provide insight into the knowledge and beliefs that Native Americans have with a mixed methods approach (Vlasin-Marty, Ritter-Gooder, \& Albrecht, 2016). The HBM allowed data collection with regards to themes surrounding the families beliefs about susceptibility and severity of foodborne illness risks (Vlasin-Marty et al., 2016).

While many health models and theories are available to serve as frameworks for research and educational programs, the HBM offers a wide range of intrapersonal focus that supports the goal to increase $\mathrm{F} / \mathrm{V}$ intakes in the middle school age population. The HBM is also frequently used to gather qualitative data as noted in focus group data collection methods (H. S. Kim et al., 2012; Mehta, Sharma, \& Lee, 2012). To substantiate all aspects of behavior change, it is proposed by this researcher, that the 
strongest framework is a study design that includes the SCLT, self-efficacy, as well as the HBM with aspects of peer influence. While several studies focus on young children and eating practices, none focus on nutrition education that is structured with the combined SCLT, self-efficacy, and HBM school based multimedia education program for middle school children with an emphasis on F/V intakes; therefore, a gap in knowledge exists. This proposed framework is presented in Figure 5. 
Figure 5: The proposed framework to improve fruit and vegetable (F/V) intakes in middle school children that combines Social Cognitive Learning Theory (SCLT), self-efficacy, and the Health Belief Model (HBM) in the program development for education sessions and focus group implementation. 


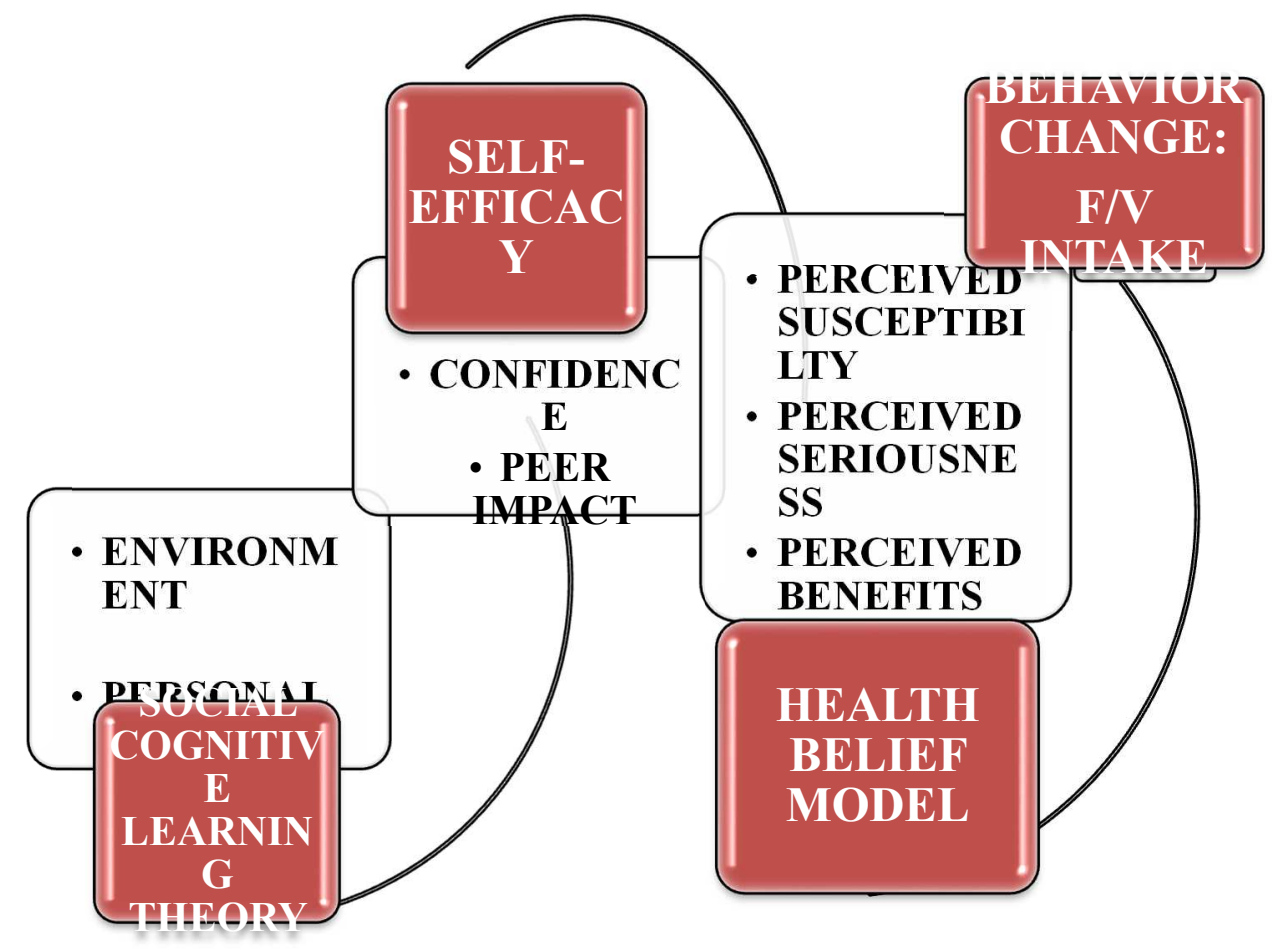




\section{CHAPTER III}

\section{METHODS}

This pilot study was quasi-experimental, with exploratory mixed-method designs that provided information needed to develop an educational tool that is focused on the importance of eating F/V. The qualitative data was necessary to first gain an understanding of the thoughts, beliefs, and general attitude about the consumption of $\mathrm{F} / \mathrm{V}$ in the diet for both the students and population in general. This data helped uncover descriptive thoughts and trends with regard to reasons $\mathrm{F} / \mathrm{V}$ are consumed or not consumed. These opinions helped give more details about how the students in this specific population view food choices, specific to $\mathrm{F} / \mathrm{V}$, and informed the research team on ways to outline the learning objectives and activities in the intervention phase.

Quantitative methods were then used to describe self-reported and parent reported intake of $\mathrm{F} / \mathrm{V}$ as well as direct observation of $\mathrm{F} / \mathrm{V}$ intake at the school lunch.

The combination of both qualitative and quantitative data, or mixed methods, was necessary to learn about dietary beliefs and practices to better understand the research problem, which then influenced the design of the study. "Mixed methods research is the collection and analysis of both qualitative and quantitative data and its integration, drawing on the strengths of both approaches" (Guetterman, Fetters, \& Creswell, 2015, p. 554). The emerging themes helped shape the curriculum in the intervention. For example, the need to incorporate more recent science-supported information such as the dietary impact on the microbiome to further explain the construct of perceived susceptibility was evident to the researchers. Other themes from the focus groups uncovered information on health benefits of consuming $\mathrm{F} / \mathrm{V}$, and this was further 
expanded in the lesson to include specific examples of health benefits such as weight management and decreased risk of heart related disease, as a result of regular $\mathrm{F} / \mathrm{V}$ consumption. The themes tied to barriers related to the consumption of $\mathrm{F} / \mathrm{V}$ were identified in the focus groups and could then be better addressed in the education sessions, as tailored examples were discussed and ways to overcome the barriers were highlighted. For example, the environment was sited as a barrier to eating F/V, and classroom discussion on how to overcome this challenge was presented, including selection of frozen F/V that last longer when limited shopping choices were available. The theory and construct of self-efficacy had a strongly supported theme emerge related to the use of recipes in order to help students gain more confidence in their personal selection of $\mathrm{F} / \mathrm{V}$. As a result, seasonal $\mathrm{F} / \mathrm{V}$ recipes were added to the curriculum plan in order to address this piece that seemed to be a very important factor according to the students enrolled in the study. The additional education, with recipes as a tool, was used to try and increase the interest of the students and potentially their confidence levels of selecting and eating $\mathrm{F} / \mathrm{V}$.

This multimedia interactive educational tool will be made available for classroom curricula in an online format to middle schools across central Indiana who have access to the Canvas online education learning system (ELS). One suburban, parochial school in Indianapolis, Indiana was recruited to participate in this efficacy trial. The study period lasted from February-May 2017. The school is a private entity of the archdioceses of the Indianapolis area, and permission was granted through the principal and support staff. School personnel and teachers provided counsel on how to best approach families and students to obtain permission from both parents and students. This research team worked 
directly with the school physical education teacher and incorporated the learning modules into the health/physical education (PE) course or study hall periods. All sixth-grade students were eligible to participate.

The Institutional Review Board (IRB), at Indiana University Purdue University at Indianapolis (IUPUI) in Indiana, reviewed and approved all methods and measures described in the research proposal (Appendix C). Informed consent forms were signed by parents to confirm their agreement to participate without penalty and to allow their child to partake in the study. Children also signed assent forms to confirm their participation without penalty (Appendix D). Parents and children were not told that the diet assessments were focused on $\mathrm{F} / \mathrm{V}$ intakes to avoid any potential influence on what was reported and observed. Student participants were given credits toward book fair purchases equivalent to ten dollars to be redeemed the following school year. A schematic drawing to further illustrate the process from recruitment to the completion of data collection points is presented in Figure 6. 
Figure 6: Schematic of data collection points including enrollment, baseline, intervention, and post intervention follow-up. 


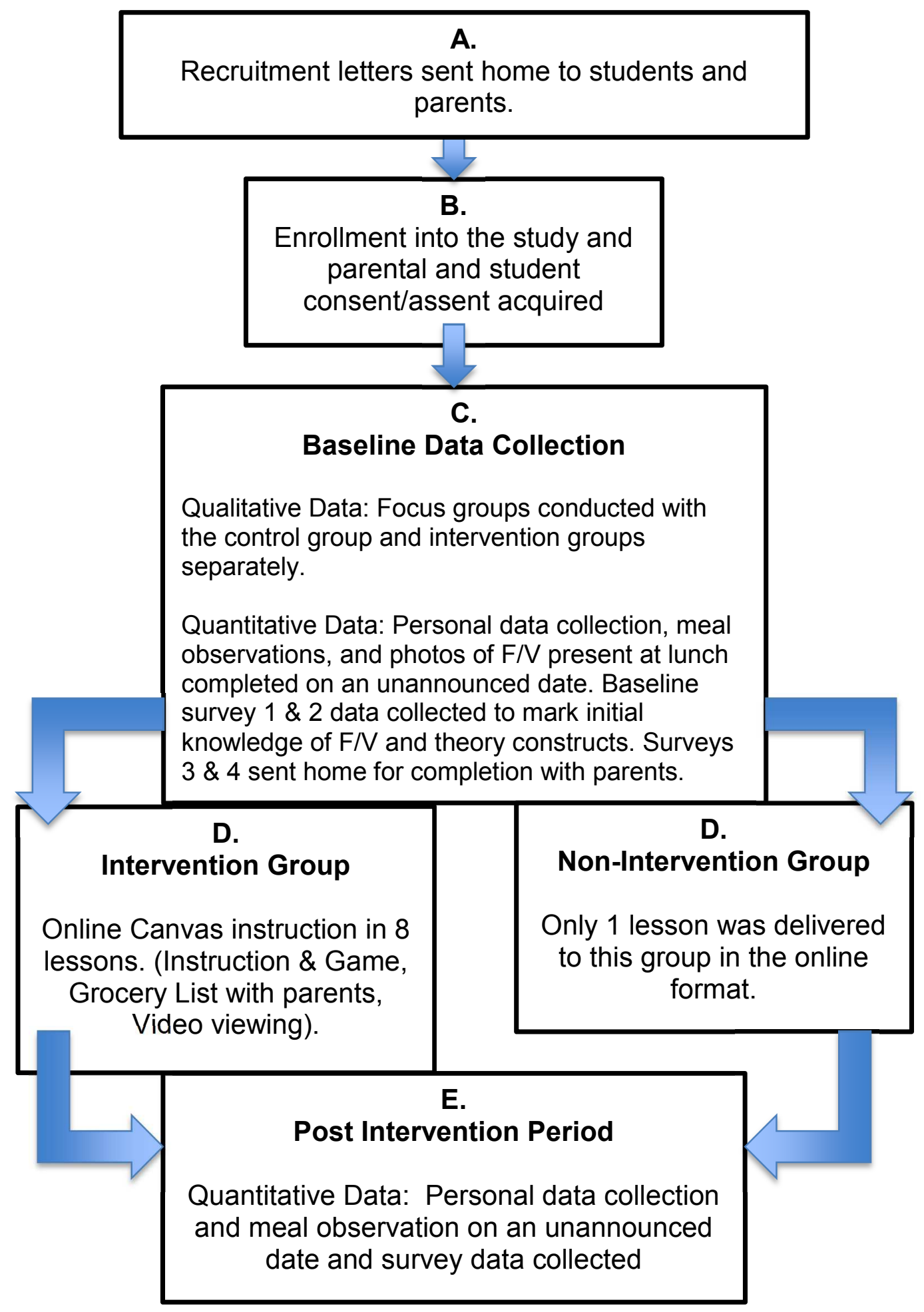




\section{INTERVENTION OVERVIEW}

Overview: The " $F \& V: 1 \bullet 2 \bullet 3$ " educational tool was designed to teach children to eat at least one half-cup serving of fruits or vegetables at breakfast, two at lunch, and three at the evening meal. The learning modules were developed within the Canvas online ELS and with the approval from the middle school teaching staff and IUPUI Department of Education, they were made available to students via guest web address invitation.

Students had personal use of school issued computers, and had the ability to individually access the online website. Written and verbal instructions on how to access the website were given to the participants during their physical education class prior to the initial lesson.

Program Intervention/Classroom Curriculum: The learning modules were delivered to students during their PE class period or study hall if extra time was required. In both groups, students were asked to bring their school assigned computers to these classes. They participated in approximately 15-20 minutes of Co-PI led educational sessions which included interactive videos, games, or trivia related to content discussed in class. Students in the intervention group, biweekly, completed the eight learning modules over a four-week period. Students in the control group received only the first lesson during the first week of the four-week period. These lessons are detailed in Table 4. A grocery store list was required for all students in the intervention group, PE class, as a general assignment. The students were asked to generate the list with their parent(s); parental signature was required to confirm both parent and child participation with the assignment. Handouts from each lesson were provided to the students to review with their parents (Appendix E). 
Table 4: The content, justification, and theoretical framework construct addressed for each of the lessons presented to middle-school children enrolled in an eight-week multimedia education program focused on the importance of fruit and vegetable intakes. 


\begin{tabular}{|c|c|c|c|}
\hline Lesson & Lesson Content & Justification & Construct \\
\hline 1 & $\begin{array}{l}\text { Fruit and vegetable }(\mathrm{F} / \mathrm{V}) \\
\text { general info for } \mathrm{F} \& \mathrm{~V}: 1 \bullet 2 \bullet 3 \\
\text { - } \text { Serving size } \\
\text { - How many cups } \\
\text { - } \text { Measure with your fist }\end{array}$ & $\begin{array}{l}\text { Myplate.gov } \\
\text { Center for Disease Control } \\
\text { (CDC) } \\
\text { National Institute of Health } \\
\text { (NIH) } \\
\text { (LaBrosse \& Albrecht, 2013) }\end{array}$ & $\begin{array}{l}\text { Social } \\
\text { Cognitive } \\
\text { Learning } \\
\text { Theory } \\
\text { (SCLT): } \\
\text { Environment } \\
\text { and } \\
\text { Personal } \\
\text { factors }\end{array}$ \\
\hline 2 & $\begin{array}{l}\text { Perceived } \\
\text { Susceptibility/Perceived } \\
\text { Seriousness } \\
\qquad \quad \text { Risks related to poor } \\
\quad \text { food choices } \\
\quad \text { Health problems }\end{array}$ & $\begin{array}{l}\text { Myplate.gov } \\
\text { CDC } \\
\text { NIH } \\
\text { (LaBrosse \& Albrecht, 2013) }\end{array}$ & $\begin{array}{l}\text { Health Belief } \\
\text { Model (HBM): } \\
\text { Perceived } \\
\text { susceptibility } \\
\text { and seriousness }\end{array}$ \\
\hline 3 & $\begin{array}{l}\text { Perceived Benefits/Perceived } \\
\text { Barriers } \\
\text { - Environment } \\
\text { - Health benefits } \\
\text { - Family impact } \\
\text { - Peer impact }\end{array}$ & $\begin{array}{l}\text { Myplate.gov } \\
\text { CDC } \\
\text { NIH } \\
\text { (LaBrosse \& Albrecht, 2013) }\end{array}$ & $\begin{array}{l}\text { HBM: } \\
\text { perceived } \\
\text { benefits and } \\
\text { perceived } \\
\text { barriers }\end{array}$ \\
\hline 4 & $\begin{array}{c}\text { Self-Efficacy/Cues To Action } \\
\text { - Practice making choices } \\
\text { - Build your own lunch } \\
\text { - Recipes to try F/V }\end{array}$ & $\begin{array}{l}\text { Myplate.gov } \\
\text { CDC } \\
\text { NIH }\end{array}$ & Self-efficacy \\
\hline 5 & Build PSA's in class & $\begin{array}{l}\text { (Gupta et al., 2013; Hong et } \\
\text { al., 2008; Rogers et al., 2016) }\end{array}$ & $\begin{array}{l}\text { SCLT: } \\
\text { Environment }\end{array}$ \\
\hline 6 & Peer Impact and repetition & $\begin{array}{l}\text { Indiana Education Standards } \\
\text { (Gupta et al., 2013; Hong et } \\
\text { al., 2008; Rogers et al., 2016) }\end{array}$ & $\begin{array}{l}\text { SCLT: } \\
\text { modeling } \\
\text { Self-efficacy } \\
\text { HBM: peer } \\
\text { influence }\end{array}$ \\
\hline 7 & Peer Impact and repetition & $\begin{array}{l}\text { Indiana Education Standards } \\
\text { (Gupta et al., 2013; Hong et } \\
\text { al., 2008; Rogers et al., 2016) }\end{array}$ & $\begin{array}{l}\text { SCLT: } \\
\text { modeling } \\
\text { Self-efficacy } \\
\text { HBM: peer } \\
\text { influence }\end{array}$ \\
\hline 8 & Peer Impact and repetition & $\begin{array}{l}\text { Indiana Education Standards } \\
\text { (Gupta et al., 2013; Hong et } \\
\text { al., 2008; Rogers et al., 2016) }\end{array}$ & $\begin{array}{l}\text { SCLT: } \\
\text { modeling } \\
\text { Self-efficacy } \\
\text { HBM: peer } \\
\text { influence }\end{array}$ \\
\hline
\end{tabular}


Each lesson was developed based on a specific health theory constructs. Lesson one was given to both the intervention and control groups and was aimed at addressing basic nutrition information and aspects of the environmental and personal factors of SCLT. In this lesson, the students learned about servings sizes and how many total servings to have specific to each meal and not just over one day. Lesson two highlighted the perceived susceptibility and perceived seriousness of health risks with low F/V intake according to the HBM. Specific issues related to poor diet were addressed including obesity, heart disease, and diabetes. The risks associated with low F/V were noted and similar to overall poor dietary inclusion. Themes that emerged from the focus groups were addressed including a healthy body weight attainment and positive messaging about foods to include in the diet versus simply foods to avoid. Lesson three informed the student about potential HBM perceived barriers and benefits of F/V consumption. Specific barriers such as lack of resources that emerged from the focus groups were addressed and ideas were provided on choices of $\mathrm{F} / \mathrm{V}$ on a limited budget. Lesson four discussed self-efficacy and presented a meal-planning game as an approach to give students more practice with F/V selections at the lunch meal. Recipes were also added as a result of the focus group sessions in order to help students build more confidence about trying new F/V. Lesson five addressed the building of public service announcements (PSA), in groups, to provide opportunities for peer influence and further practice with the F/V information presented as aligned with the SCLT. Lessons six, seven, and eight consisted of PSA presentations by the students for enhanced peer reporting, influence, and modeling as outlined by the SCLT and HBM. 


\section{DATA COLLECTED}

Demographic: All data collection points and interventions are presented in Table 5. Demographic data of age, gender, and ethnicity was collected as part of the "Farm to Table Study" (Survey 1) in an online format during the student's PE class or study hall at baseline and post study. These data are part of what the social cognitive learning theory (SCLT) addresses as personal factors.

Classroom Focus Group: Qualitative data collection was selected as a means of obtaining information to better understand the research problem and reasons why food selections are made in this select population (Creswell, 2008). A baseline focus group was conducted separately for both the control and intervention groups and audio was recorded using the application available on the iPad labeled as "recorder". The groups were separated in order to keep the size of the focus group smaller to elicit more information (Creswell, 2008, p. 226). Questions used in the focus group were scripted and approved by the research team, educators, and IRB prior to the recorded sessions with the students; the questions are listed in Table 6. Previous focus group research based on dietary soy intake and constructs of the HBM were used as a guide for questions and follow up prompting (Robinson, Bielamowicz, Rodgers, Wong, \& Konzelmann, 2008). The focus group incorporated all the theory framework constructs: SCLT, self-efficacy, and HBM constructs (Appendix F). The information gathered from these focus groups was used to inform the research team how to proceed with educational planning and development. Subsequently, this information changed the intervention to include more information geared toward increasing self-efficacy including the addition of recipes to help with variety and taste enhancement. 
Qualitative data included words and phrases and the text was organized according to themes that were identified to describe the "central phenomenon" identified in the study (Creswell, 2008, p. 57). The constructs of the health theory framework served as guidelines for organizing main ideas that emerged in the focus group discussion in each group. These constructs included: (1) perceived susceptibility of poor health related to lack of F/V in diet, (2) perceived severity of poor health related to lack of F/V in diet, (3) perceived benefits of $\mathrm{F} / \mathrm{V}$ in the diet, (4) cues to action for health promotion and disease prevention. This research team completed transcriptions of both focus group sessions, and then several reviews of the information were completed. The statements were colorcoded and highlighted depending on the theme they corresponded with under each construct previously noted.

\section{DATA RELATED TO STUDY AIMS}

AIM 1: To determine if an educational intervention, focused on the importance of $F / V$ in the diet and administered to middle school age children (11-12 years) who attend a parochial Indianapolis-area school, impacts (1) the amounts of F/V included in and consumed from home-prepared school lunch; (2) the students' attitudes toward F/V intake; (3) the students' knowledge of F//V benefits; and (4) the students' selfefficacy/perception related to eating $\mathrm{F} / \mathrm{V}$. 
Table 5: Time frame for the study implementation activities and data collection with supported theory construct. 


\begin{tabular}{|c|c|c|c|c|c|c|c|c|c|c|}
\hline \multirow[b]{2}{*}{ Study Event } & \multirow{2}{*}{$\begin{array}{c}\text { Theory } \\
\text { Construct }\end{array}$} & \multicolumn{9}{|c|}{ Study Weeks } \\
\hline & & 1 & 2 & 3 & 4 & 5 & 6 & 7 & 8 & 9 \\
\hline Consent/Assent & & $X^{*}$ & & & & & & & & \\
\hline & & \multicolumn{9}{|c|}{ Data Collection } \\
\hline Age, gender, ethnicity & SCLT $^{* *}$ & $X^{*}$ & & & & & & & $\mathrm{X}^{*}$ & \\
\hline Survey 1 & SCLT & & & $\mathrm{X}^{*}$ & & & & & $\mathrm{X}^{*}$ & \\
\hline Survey 2 & SCLT & & & $\mathrm{X}^{*}$ & & & & & $\mathrm{X}^{*}$ & \\
\hline Survey 3 & SCLT & & & $\mathrm{X}^{*}$ & & & & & & \\
\hline Survey 4 & SCLT & & & $\mathrm{X}^{*}$ & & & & & & \\
\hline $\begin{array}{l}\text { Height, Weight, BMI, } \\
\text { Blood pressure }\end{array}$ & SCLT & & & $\mathrm{X}^{*}$ & & & & & & $\mathrm{X}^{*}$ \\
\hline Focus Group & $\begin{array}{c}\text { SCLT } \\
\text { Self-Efficacy } \\
\text { HBM }^{* * *}\end{array}$ & & & $\mathrm{X}^{*}$ & & & & & & \\
\hline Meal Observation & $\begin{array}{l}\text { Behavior } \\
\text { Change }\end{array}$ & & & $\begin{array}{l}\mathrm{X}, \mathrm{X}, \\
\mathrm{X}^{*}\end{array}$ & & & & & & $X^{*}$ \\
\hline Lesson 1 & SCLT & & & & $\mathrm{X}^{*}$ & & & & & \\
\hline Lesson 2 & HBM & & & & & $\mathrm{X}$ & & & & \\
\hline Lesson 3 & HBM & & & & & $\mathrm{X}$ & & & & \\
\hline Lesson 4 & Self-efficacy & & & & & & $\mathrm{X}$ & & & \\
\hline Lesson 5 & SCLT & & & & & & $\mathrm{X}$ & & & \\
\hline Lesson 6 & $\begin{array}{c}\text { SCLT } \\
\text { Self-efficacy } \\
\text { HBM }\end{array}$ & & & & & & & $\mathrm{X}$ & & \\
\hline Lesson 7 & $\begin{array}{c}\text { SCLT } \\
\text { Self-efficacy } \\
\text { HBM } \\
\end{array}$ & & & & & & & $\mathrm{X}$ & & \\
\hline Lesson 8 & $\begin{array}{c}\text { SCLT } \\
\text { Self-efficacy } \\
\text { HBM }\end{array}$ & & & & & & & & $\hat{X}$ & \\
\hline
\end{tabular}

*Completed in both intervention and control groups

**Social Cognitive Learning Theory

***Health Belief Model

$\wedge^{\wedge}$ Education intervention completed 
Table 6: Selected discussion probes and questions used in the focus groups to identify the health belief model (HBM) constructs of perceived severity, perceived benefits, perceived barriers, cues to action, and self-efficacy as they relate to fruit and vegetable intake (Robinson et al., 2008). 


\begin{tabular}{|c|c|}
\hline $\begin{array}{c}\text { Health Belief Model Construct } \\
\text { related to } \mathrm{F} / \mathrm{V} \text { intake }\end{array}$ & Selected Discussion Probes* \\
\hline $\begin{array}{c}\text { Perceived susceptibility of poor } \\
\text { health related to lack of fruits and } \\
\text { vegetables in your diet }\end{array}$ & $\begin{array}{l}\text { Why do you think some people eat healthy } \\
\text { and why do some people not eat healthy } \\
\text { foods? } \\
\text { How do eating fruits and vegetables help } \\
\text { someone to eat healthy? } \\
\text { Can you describe health problems that } \\
\text { might occur from not eating healthy? }\end{array}$ \\
\hline $\begin{array}{l}\text { Perceived severity of poor health } \\
\text { related to low intakes of fruits and } \\
\text { vegetables }\end{array}$ & $\begin{array}{l}\text { If you choose to eat fruits and vegetables, } \\
\text { can you describe why you eat them? }\end{array}$ \\
\hline $\begin{array}{l}\text { Perceived benefits of fruits and } \\
\text { vegetables }\end{array}$ & $\begin{array}{l}\text { Do you go food shopping with your } \\
\text { parents? } \\
\text { If you buy fruits and vegetables when you } \\
\text { go shopping describe what they are? Why } \\
\text { or why not? }\end{array}$ \\
\hline $\begin{array}{c}\text { Cues to Action } \\
\text { for health promotion and disease } \\
\text { prevention }\end{array}$ & $\begin{array}{l}\text { Some people do not eat any fruits and } \\
\text { vegetables or they eat low amounts. Can } \\
\text { you tell me what might happen to their } \\
\text { health? } \\
\text { What can they do to help prevent any health } \\
\text { problems? }\end{array}$ \\
\hline
\end{tabular}

*Focus group questions adapted from "Bone and Soy" focus group based on the HBM (Robinson, Bielmowicz, Rodgers, Wong \& Konzelmann, 2008). 
Estimates of F/V Intake; Survey Data: Data related to F/V intake were obtained from four surveys; students at school at baseline and post study completed surveys 1 and 2 and surveys 3 and 4 were completed by parents at home at baseline only and returned to the school with the student. Surveys 1, 3, and 4 were adapted to include constructs related to SCLT, self-efficacy, and HBM as well as new information regarding the benefits of F/V. These surveys are described below and the actual surveys are presented in the Appendix G.

Survey 1 is an adapted version of the validated "Farm to Table" survey (Baranowski et al., 2000; Jamelske, Bica, McCarty, \& Meinen, 2008; Pliner \& Hobden, 1992). This survey focuses on attitudes related to $\mathrm{F} / \mathrm{V}$ intakes, knowledge about the benefits of $\mathrm{F} / \mathrm{V}$, self-efficacy/perception of eating behaviors related to $\mathrm{F} / \mathrm{V}$ and included a 24-hour dietary recall screener of F/V intakes. The adapted version of this validated tool includes the addition of four questions that reflected new information regarding health benefits of $\mathrm{F} / \mathrm{V}$ information that was delivered in the lessons in the intervention group.

Survey 2 is the Day in the Life Questionnaire (DILQ) for children (Edmunds \& Ziebland, 2002). The questionnaire prompts students to recall their food, and beverage intakes as well as their activities at designated times during the previous day. Another aspect of this survey invites students to draw a picture of their lunch. At baseline the survey included the timeframe of breakfast through afternoon snack. At the study end, the timeframe included a recall of the entire day; breakfast through evening snack.

Survey 3 is an adapted version of the validated Eating at America's Table Study (EATS) (LaBrosse \& Albrecht, 2013; Murphy, White, Viani, \& Mosby, 2016; Thompson et al., 2010). At baseline, the questions were adapted: (1) to ask about the foods the parents 
offered to the child and (2) the amount consumed by the child. The survey was sent home with the students for their parents to complete and return the following week. The questions allowed parents to indicate the types and amounts of $\mathrm{F} / \mathrm{V}$ servings offered to their children as well as the amounts consumed by their children; responses were estimated as servings in a one-month period and then calculated as a mid-point frequency and serving amount to total a daily average. Questions were adapted to identify the foods parents offered to the child and the amount the child would consume.

Survey 4 is an abbreviated version of the validated 2012 Youth Adolescent Food Frequency Questionnaire (YAQ) (Borradaile et al., 2008), and was sent home with students, at baseline, to gather parent responses about the F/V intakes of their child. The survey required intake estimates within a weekly time frame as well as a monthly time frame to include foods from the F/V sub groupings and fiber/grain group. Average daily estimated intakes were calculated for F/V servings and subgroupings within the following food groups: fruits, vegetables, potatoes, grains containing fiber, and legumes.

Estimates of F/V Intake, Lunch Observations: Student lunches brought from home, were assessed for $\mathrm{F} / \mathrm{V}$ servings on three different unannounced days at baseline and on two different unannounced days post-study on a Tuesday, Wednesday or Thursday. Mondays and Fridays were not chosen because hot lunches were purchased and served from outside food vendors on these days. The procedure on days when students carry their lunch is that the packed lunches are collected in the classroom in baskets, brought to the lunchroom, and distributed as students enter the lunchroom. On the observation days, when lunches were still in the classroom, participating students attached a sticker tag with his or her assigned study ID number to their lunch container. These lunches were brought 
to a clean area where trained research registered dietitians observed the contents of each lunch. All food items were removed from the lunch container, with gloved hands, and arranged on a disposable plate next to a ruler for an accurate scale of measurement. The sticker tag with the ID number was placed near the plate and the plated lunch was digitally photographed with an iPad device; these pictures were used to double-check the recorded data. The following data for each participant was recorded on a form: (1) presence of $\mathrm{F} / \mathrm{V}$ (yes or no); (2) type of F/V; (3) approximate number of $\mathrm{F} / \mathrm{V}$ servings in half-cup serving sizes or 1 cup leafy greens (USDA, 2017). Foods were replaced in the lunch container and the ID tag was again adhered to the container. At lunchtime, each participant was given a large disposable plate with his or her lunch and instructions to leave any food that was not eaten on the plate with their ID number tag, and return the plate to the table where the research team was located. Observers recorded, on the same data collection form, the percentage of consumption of F/V servings. Any student, who wanted to keep any uneaten food items, was given that option after their plate was observed.

Aim 2: To determine if personal factors such as BMI, blood pressure, and gender relate to the amounts and types of F/V included in and consumed from home-prepared school lunches among middle school children ages 11-12 years.

Personal Factors Data Collection: Student height, weight and blood pressure were collected in a private area during the student's study hall period at baseline and repeated post study. Students remained clothed, however sweaters, jackets and shoes were removed. Students were asked to stand erect on a health care grade scale (Detecto $\odot$ ) and height in inches was noted when a horizontal metal strip attached to a tape measure was 
brought to the crown of the head and weight in pounds was noted and recorded. Two individual measures for both weight and height were taken. (R. S. Gibson, 2005). Body mass index (BMI) was calculated from height and weight measures and z-scores were determined using the US standardized data method for growth assessment (CDC, 2016, 2017; Wells \& Fewtrell, 2006). Blood pressure measures were obtained with digital blood pressure machines (Kelly et al., 2015; NIH, 2007; Setayeshgar et al., 2017). If a student's blood pressure was found to be elevated ( $\geq 120 / 80 \mathrm{mmHg}$ ), the child's parent would have been notified by telephone to make them aware of this finding the same day and contact with the child's primary care physician would have been suggested.

Parental involvement: Parents were asked to complete the baseline data surveys 3 and 4 . Students assigned to the intervention group were required to complete a grocery list with their parent and obtain a signature from their parent to verify that the parent was involved in the process. Parents were also asked to review the handouts sent home with their child that included bulleted information similar to the content provided in the web-based lessons.

\section{MEASURES}

Statistical Analyses: This is a pilot study and results were used to help determine the sample sizes needed for future like studies. Data was analyzed with SPSS Statistics 24. The statistical methods that were used include descriptive statistics, paired t-tests, analysis of variance (ANOVA), Pearson coefficient of correlation, and qualitative analysis; these are listed in Table 7 alongside the respective variable measure. The statistical power, or the alpha level of the study, will have a probability set at 0.05 and a confidence interval of 95 percent. We anticipate statistical significance to be determined 
with at least 12 subjects in each group. Any possible deviations from significance may be due to a small population or brevity of the study protocol; therefore, $p$ values are reported to identify possible trends towards significance.

Calculations for Observed Meals: Data collected on three separate unannounced days at baseline were averaged for fruit servings, vegetable servings, total of both F/V servings, and the percent amount consumed. The number of servings consumed was calculated from the percent consumed of the fruit servings, vegetable servings and F/V servings that were observed in the packed lunches. Data from the three days at baseline were tested for variance and it was decided that only one day of meal observation was required for the measure at the end of the study.

Scores for Attitude, Knowledge, Self-Efficacy/Perception: Three sets of questions were adapted from the "Farm to Table" survey, (Appendix H) and responses were assigned number values. When added together, a final score for each of the categories (knowledge, attitude and self-efficacy/perception) was generated for each participant at baseline and again post study. These final scores were used to compare the groups to determine the effect of the education intervention.

There are 11 questions on the knowledge portion of the survey and they were scored for correct responses. Four questions were added to this section that reflected the content of the lessons more specifically to F/V benefits. These questions included: (1) What is a benefit of eating F/V? (2) What is the role of fiber from foods?; (3) How would you best describe the word microbiota?; (4) What helps improve the health of your gut or intestines?. A correct response was scored with one point, and an incorrect was marked with a zero; the final score was the sum of all the points earned. 
Table 7: The variables measured in this research study and the complimentary statistical test used to assist with interpretation of the results. 


\begin{tabular}{|l|c|}
\hline \multicolumn{1}{|c|}{ Variable Measure } & Statistical Test \\
\hline Demographic information (baseline and post study: & Descriptive Statistics \\
blood pressure) & \\
\hline $\begin{array}{l}\text { Farm to Table Survey 1: Baseline/Post Study Attitude, } \\
\text { Knowledge, Self-Efficacy/Perception, Dietary intake } \\
\text { screener and relative intake }\end{array}$ & Paired T-Test \\
\hline $\begin{array}{l}\text { DILQ Survey 2: Baseline /Post study student 24-hour } \\
\text { DILQ recall of intake breakfast-afternoon snack; average } \\
\text { only of post breakfast-dinner }\end{array}$ & (ANOVA) \\
\hline $\begin{array}{l}\text { EATS Survey 3-parent reported monthly report of F/V } \\
\text { food offerings }\end{array}$ & Paired T-Test \\
\hline $\begin{array}{l}\text { YAQ-W/YAQ-M Survey 4-parent reported dietary intake } \\
\text { of fiber/grains, fruits, vegetables by child in one week and } \\
\text { month }\end{array}$ & Means for both groups \\
\hline $\begin{array}{l}\text { Meal observations: Baseline / Post Study fruit servings, } \\
\text { vegetable servings, F/V combined servings at school } \\
\text { lunch meal, consumption }\end{array}$ & Variance at baseline, \\
\hline Focus Group & ANOVA \\
\hline Comparison of observations and surveys & Pearson Correlation \\
\hline
\end{tabular}


The attitude portion is comprised of 20 questions. Response to some questions were assigned the following number values: "a lot" $=4$, "a little" $=3$, "not very much"=2, and "not at all"=1. Other questions were assigned the following number values: "definitely"=4, "probably"=3, "probably not"=2, "definitely not"=1. The final two questions asked about how many times a student tried F/V that were new since the school year started. Responses were assigned the following number values: "never"=1, "1 time" $=2$, "2 times" $=3, " 3$ times" $=4$, "at least 4 times" $=5$. The total of all these scores were added up for a final score between 20-82. The higher the number indicated a higher, positive attitude toward eating F/V foods.

The section that measured self-efficacy/perception of eating F/V included nine questions. The responses were assigned number values as follows: "yes, all of the time/very likely"=2; "yes, sometimes/likely"=1; "no/not likely"=0. This portion of the survey also included four questions regarding the participant's ability to make healthy choices. The responses were assigned a number value as follow: "I know I can"=4; "I think I can"=3, "I'm not sure I can"=2; "I know I can't"=1. The numbers were added to determine a response final total score of 6-30. The higher number indicating a higher level of self-efficacy or confidence related to eating and selecting F/V in their diet. F/V Screener and Relative Amounts: A 24-hour F/V screener was included in the adapted "Farm to Table" survey. The 13 questions were designed to ask students if they consumed any F/V groupings including: fruit, vegetables, legumes, and potatoes. The selection choices were yes or no; "yes"= 1 and "no" $=0$. A percentage of the entire group that responded "yes" to consuming foods was calculated for each food grouping. If the participant answered, "yes" to a specific food, they were asked to identify if they 
consumed: "a little", "some", or "a lot". A relative amount was assigned to each student response, for each food item sub group, according to an algorithm; a score for each food response ranged from "a little" $=0.25-1.0$; "some" $=0.5-2.0$; or "a lot" $=2.0-3.0$ depending on the food item (for example if they ate applesauce and marked it as "a little" then if received a relative amount of 0.25 servings). The relative amount was approximately equal to a $1 / 2$ cup serving size for F/V portions. ("Tool: Knowledge, attitude, and consumption behavior survey," 2010).

Day in the Life Questionnaire (DILQ)-Survey 2: The DILQ survey includes a 24-hour dietary recall completed by participants in writing and by drawing pictures, at baseline and again post study. The scope of data collection at the two time points differed somewhat. At baseline, recalled information from the previous day was captured from breakfast through the afternoon snack. Whereas, the post study evaluation included a recall of the entire previous day; breakfast through the afternoon snack as well as dinner and after dinner intake. Therefore, the intervention effect from baseline to post study compared F/V intakes generated from breakfast through the afternoon snack. Another score of $\mathrm{F} / \mathrm{V}$ intake during the entire previous day was generated for both groups, however only at the post study time point. Scores were generated from the assigned number values; a value of " 1 " was assigned each time a F/V or $100 \%$ juice was mentioned in the survey. Food items, other than F/V or $100 \%$ juice were assigned a value of " $0 . "$ A total value was calculated as the sum of all numbers on the survey and was considered roughly equivalent to the number of $\mathrm{F} / \mathrm{V}$ servings. A paired T-test was used to compare the baseline and post study breakfast through afternoon snack changes within and between the groups. The mean post study scores that included F/V intake data 
reflective of breakfast through after dinner were compared between the groups with a ttest.

Scoring for the Adapted EATS Study-Survey 3: Parents were asked to complete this survey at baseline. Within the survey, they reported what $\mathrm{F} / \mathrm{V}$ servings were offered to their child over a month's time, as well as the usual portion size that the child would eat; responses were used in an equation that estimated F/V servings per day. Food categories included: juice, fruit in units, fruit in cups, lettuce in cups, French fries, other white potatoes, dried beans, other vegetables, tomato sauce, and vegetable soups; an average serving for each food category was compared to the Food Guide Pyramid for average servings according to the National Institute for Health (NIH) (NIH, 2016). This survey was scored according to the guidelines set forth by the NIH and scoring procedures for the "All-Day Screener." The frequency response questions pertaining to the number of times per month/week/day F/V were offered were scored with number values of 0.0-5.0 depending on one of 10 selections possible: "never", "1-3 times per month", "1-2 times per week”, "3-4 times per week”, "5-6 times per week”, “1 time per day”, "2 times per day", "3 times per day", "4 times per day", "5 or more times per day". The assigned F/V specific to juice, fruit in units, fruit in cups, lettuce salad, French fries, other white potatoes, dried beans, other vegetables, tomato sauce, and vegetable soups were scored according to a portion size category of 1-4. The generated number values were added together to determine F/V servings for each individual food category (NIH, 2016); mean scores from the intervention and control groups were compared with a t-test. 


\section{Scoring for Adapted Youth Adolescent Food Frequency Questionnaire (YAFFQ or}

YAQ)-Survey 4: Parents were asked to complete this survey at baseline. The survey contained questions for parents about the child's diet in review of a week's intake and again over a one-month period. Scores for the weekly review were calculated based on the food categories including: fruits, vegetables, potatoes, fiber/grains, and legumes. A number value was assigned based on the following: "less than $1 /$ week" $=1$; "once a week" = 2, "2-3 times per week" = 3, "4-6 times per week" = 4; "once a day" = 5, " $2+$ a day" $=6$. These values were summed for each food sub grouping (fiber/grains sub group was excluded) and then divided by 7 to determine an average daily intake of F/V servings. The monthly screener was also scored in a similar way. Each of the 54 questions was assigned to a F/V subgroup. The responses included: "never" = 0, "1-3 times per month" = 1, "once per week" = 2,"2-4 per week" = 3, "more than 4 per week" $=4$. These numbers were added (fiber/grains sub group was excluded) then divided by 30 days to estimate a daily average of F/V servings. 


\section{CHAPTER IV}

\section{RESULTS}

There were 30 parent-child dyads enrolled in this school-based study. The current analysis includes 29 dyads as one student was at risk of bias assessment. Therefore, the current analysis included data from 29 student participants and their parents. Each student was enrolled in one of two classes in the sixth grade; one class received the intervention, and the other served as the control group. The eight-lesson education program was delivered primarily during the physical education (PE) class; therefore the class assigned to receive the intervention had the greater number of remaining scheduled PE class periods. All statistical tests are presented in Appendix I and the raw data is in Appendix J.

\section{PARTICIPANT PERSONAL FACTORS}

Demographic data for all participants are presented in Table 8 and include: age, gender, and ethnicity. All students ranged in age from 11-12 years and the mean age was $11.83 \pm 0.38$ years. There were 16 ( 8 males and 8 females) students in the intervention group and 13 (7 males and 6 females) students in the control group. The ethnicity of the entire group was Caucasian 81\%, American Indian 4\%, and Other 7\%. 
Table 8: Age, gender, and ethnicity of middle school children enrolled in an eight-week multimedia educational program focused on the importance of fruit and vegetable intakes. 


\begin{tabular}{|c|c|c|c|}
\hline Characteristic & $\begin{array}{l}\text { Intervention } \\
\text { Group } \\
\mathrm{N}=16\end{array}$ & $\begin{array}{c}\text { Control } \\
\text { Group } \\
\mathbf{N}=13\end{array}$ & $\begin{array}{c}\text { Both } \\
\text { Groups } \\
\text { N=29 } \\
\text { Mean } \pm \text { SD* }\end{array}$ \\
\hline $\begin{array}{l}\text { Age, years } \\
\text { Number } \sim 11 \text { years } \\
\text { Number } \sim 12 \text { years }\end{array}$ & $\begin{array}{c}11-12 \\
3 \\
13\end{array}$ & $\begin{array}{c}11-12 \\
2 \\
11 \\
\end{array}$ & $11.83 \pm 0.38$ \\
\hline $\begin{array}{l}\text { Gender } \\
\qquad \begin{array}{l}\text { Number }=\text { Male } \\
\text { Number }=\text { Female }\end{array}\end{array}$ & $\begin{array}{l}8 \\
8\end{array}$ & $\begin{array}{l}7 \\
6\end{array}$ & $\begin{array}{l}15 \\
14\end{array}$ \\
\hline $\begin{array}{l}\text { Ethnicity } \\
\text { Number }=\text { Caucasian } \\
\text { Number }=\text { American Indian } \\
\text { Number }=\text { Other }\end{array}$ & $\begin{array}{c}13 \\
1 \\
2\end{array}$ & $\begin{array}{c}11 \\
0 \\
1\end{array}$ & $\begin{array}{c}24 \\
1 \\
3\end{array}$ \\
\hline
\end{tabular}

*Standard deviation 


\section{Focus Group: Personal and Environmental Factors}

Focus group discussions were conducted with each group separately at the beginning of the study and were transcribed. Data were analyzed once quantitative statistics were conducted to then determine key themes that may further expand on the constructs of the HBM and self-efficacy. Distinct themes emerged from the focus group discussions that aligned with each HBM and self-efficacy construct category. These were reviewed and summarized separately for each group, intervention and control. In addition, combined findings from both the intervention and control groups were reviewed and are included in Table 9. Information gathered from the focus groups impacted the curriculum design to then include recipes as part of the educational component in the lesson modules.

Within the perceived susceptibility construct, most notable themes were taste preferences and health benefits as it was repeatedly stated in both groups. Within the perceived severity construct, comments reflected those made within the susceptibility construct, reiterating more disease risks could occur if $\mathrm{F} / \mathrm{V}$ intake is too low. Examples of weight management, low energy levels, and cancer risks were also mentioned as possible negative outcomes related to poor $\mathrm{F} / \mathrm{V}$ intake and increased severity of health conditions. While the benefits and barriers also related to disease prevention, further comments were highlighted that reflected concerns about weight management, having improved energy, and disease prevention:

"Some fruits and vegetables can protect you against diseases so if you don't eat those it might be easier to get that disease." (Boy, age 12). 
Table 9: Identified themes for each construct of the health belief model (HBM) and selfefficacy that emerged during focus group discussions conducted at baseline with middle school children enrolled in an eight-week multimedia educational program focused on the importance of fruit and vegetable intakes. 


\begin{tabular}{|c|c|c|c|}
\hline $\begin{array}{l}\text { Health Belief } \\
\text { Model } \\
\text { Construct }\end{array}$ & $\begin{array}{c}\text { Intervention Group } \\
\text { Themes }\end{array}$ & $\begin{array}{c}\text { Control Group } \\
\text { Themes }\end{array}$ & $\begin{array}{l}\text { Both Groups } \\
\text { Themes }\end{array}$ \\
\hline $\begin{array}{l}\text { Perceived } \\
\text { susceptibility of } \\
\text { poor health } \\
\text { related to lack of } \\
\text { fruits and } \\
\text { vegetables in } \\
\text { your diet }\end{array}$ & $\begin{array}{l}\text {-Taste preferences impact } \\
\text { food choices } \\
\text {-Health Benefits to eating } \\
\text { healthy } \\
\text {-Live longer with eating } \\
\text { healthy } \\
\text {-Job requirement }\end{array}$ & $\begin{array}{l}\text {-Prefer tasteful } \\
\text { foods } \\
\text {-Cost } \\
\text {-Health Benefits }\end{array}$ & $\begin{array}{l}\text {-Taste } \\
\text { preferences } \\
\text { impact food } \\
\text { choices that } \\
\text { may cause } \\
\text { poor food } \\
\text { choices } \\
\text {-Health benefits }\end{array}$ \\
\hline $\begin{array}{l}\text { Perceived } \\
\text { severity of poor } \\
\text { health related to } \\
\text { low intake of } \\
\text { fruits and } \\
\text { vegetables }\end{array}$ & $\begin{array}{l}\text {-When you eat junk more } \\
\text { often you crave it more } \\
\text { often. } \\
\text {-Some F/V can protect you } \\
\text { against diseases } \\
\text {-Cancer risks }\end{array}$ & $\begin{array}{l}\text {-Healthy foods } \\
\text { allow you to stay } \\
\text { fit and do more } \\
\text { physical activities } \\
\text {-Live longer } \\
\text {-Appearance } \\
\text {-Weight } \\
\text { management } \\
\text {-Improved energy } \\
\text {-Provide vitamins }\end{array}$ & $\begin{array}{l}\text {-More Disease } \\
\text { risk } \\
\text {-Weight } \\
\text {-Energy } \\
\text {-Cancer } \\
\text { risk increased }\end{array}$ \\
\hline $\begin{array}{l}\text { Perceived } \\
\text { benefits of fruits } \\
\text { and vegetables }\end{array}$ & $\begin{array}{l}\text {-Prevention of cancer } \\
\text {-More energy for activity } \\
\text {-Delivery of vitamins }\end{array}$ & $\begin{array}{l}\text {-Prevention of } \\
\text { cancer } \\
\text {-More energy to } \\
\text { run more }\end{array}$ & $\begin{array}{l}\text {-Prevention of } \\
\text { cancer } \\
\text {-More energy for } \\
\text { activity }\end{array}$ \\
\hline $\begin{array}{l}\text { Perceived } \\
\text { barriers of } \\
\text { consuming fruits } \\
\text { and vegetables }\end{array}$ & $\begin{array}{l}\text {-Taste helps with eating } \\
\text { more } \mathrm{F} / \mathrm{V} \\
\text {-Parents make me eat } \\
\text { them }\end{array}$ & $\begin{array}{l}\text {-Yes, shopping } \\
\text { with parents } \\
\text {-All types of } \mathrm{F} / \mathrm{V} \\
\text { purchased }\end{array}$ & $\begin{array}{l}\text {-Taste } \\
\text {-Environment }\end{array}$ \\
\hline $\begin{array}{l}\text { Cues to action } \\
\text { for health } \\
\text { prevention }\end{array}$ & $\begin{array}{l}\text {-High cholesterol may } \\
\text { occur when you don't } \\
\text { eat F/V } \\
\text {-Cancer may occur when } \\
\text { you don't eat F/V }\end{array}$ & $\begin{array}{l}\text {-High Cholesterol } \\
\text { may occur if diets } \\
\text { are low in } \mathrm{F} / \mathrm{V} \\
\text {-Cancer may } \\
\text { occur }\end{array}$ & $\begin{array}{l}\text {-High cholesterol } \\
\text { could occur } \\
\text {-Cancer could } \\
\text { occur }\end{array}$ \\
\hline $\begin{array}{l}\text { Self-efficacy for } \\
\text { consuming fruits } \\
\text { and vegetables }\end{array}$ & $\begin{array}{l}\text {-More options and } \\
\text { improved taste might } \\
\text { increase intake } \\
\text {-If you put the with } \\
\text { something else you } \\
\text { might grow to like them }\end{array}$ & $\begin{array}{l}\text {-Recipes help to } \\
\text { learn how to put } \\
\text { F/V with } \\
\text { something else } \\
\text {-Grow to like } \\
\text { them; improve the } \\
\text { taste. }\end{array}$ & $\begin{array}{l}\text {-More options } \\
\text {-Grow to like - } \\
\text { them } \\
\text {-Improvement to } \\
\text { taste }\end{array}$ \\
\hline
\end{tabular}


"Some people would eat healthy foods because they know it will benefit them in the activities that they do, because they know it will keep them energized instead of eating foods that are not as good for you." (Girl, age 11)

"I think I eat them, because personally I think I like the taste of fruits and vegetables and I'm fine with eating them, but I also think... I think about (how) I probably should be eating fruits and vegetables with how much activity I do. I probably need something like nutritious to keep me going." (Girl, age 12)

The cues to action construct revealed themes again associated with the reduction of risks associated with high cholesterol and cancer as triggers to make behavior changes, especially if F/V intake is low in the diet. Self-efficacy measures for the consumption of F/V were formulated around how recipes might impact changes in F/V intakes. Students in both groups mentioned that recipes would improve the taste of $\mathrm{F} / \mathrm{V}$ and provide more food choices; these comments were associated with the theme "growing to like F/V". Because of these comments, recipes for $\mathrm{F} / \mathrm{V}$ were included as part of the educational intervention; recipes were chosen based on taste, high nutrient density, and the likelihood that they would increase F/V servings. Participants stated:

"Sometimes also I don't know if this is just me, but you feel more proud and want to eat it if its something that you made and might be motivation for people if they had a lot of recipes too." (Girl, age 12)

"I think with recipes that have fruits and vegetables like on their own or if you put it in a sandwich or I don't know, then you might grow to like them, the fruit and vegetable part. " (Boy, age 12)

"...I think that when you put fruits and vegetables with other things there a lot more options and with only just fruits and vegetables there are a lot less, because there are only certain kinds of fruits and vegetable. So when you put them with recipes you can have a lot more choices of things you like." (Girl, age 12) 
"Like if you have more recipes, you have more selection, and like choices and if you don't like a certain vegetable maybe its in a dish that you really like, maybe you could try it and you didn't know it could be done." (Girl, age 12). 


\section{RESULTS AS THEY RELATE TO STUDY AIMS}

AIM 1: To determine if an educational intervention, focused on the importance of $F / V$ in the diet and administered to middle-school aged children (11-12 years) who attend a parochial Indianapolis-area school, impacts: (1) the amounts of F/V included in and consumed from home-prepared school lunches; (2) the students' attitudes toward F/V intake, (3) the students' knowledge of F/V benefits; and (4) the students' selfefficacy/perception related to eating $\mathrm{F} / \mathrm{V}$.

Lunch Observation of F/V Servings: The estimated numbers of F/V servings observed in home prepared school lunches of middle school children enrolled as participants in an eight-week multimedia education program focused on the importance of $F / V$ in the diet are included in Table 10. At baseline, the number of fruit servings was $1.01 \pm 0.54$ and the number of vegetable servings was $0.33 \pm 0.60$ in the intervention group, and $1.11 \pm$ 0.46 fruit servings and $0.21 \pm 0.31$ vegetable servings were observed in the control group. Post study observations were collected on two separate days because on the first observation day, the intervention group was awarded with a pizza party in an alternative eating area other than the cafeteria and seven students did not bring a lunch. Therefore, the observations on this day were excluded from both groups and observations from another post study day were those used as the post measure in the analysis of the intervention effect on $\mathrm{F} / \mathrm{V}$ servings present in and consumed from lunches. The post study amount of fruit servings observed in the lunches in the intervention group, was 1.30 \pm 0.93 servings and $1.02 \pm 0.65$ servings in the control group. 
Table 10: The estimated number (\#) of fruit and vegetable $(\mathrm{F} / \mathrm{V})$ servings observed (baseline and post study) in home packed school lunches of middle school children enrolled in an eight-week multimedia educational program focused on the importance of fruit and vegetable intakes. 


\begin{tabular}{|c|c|c|c|c|c|c|c|c|c|}
\hline \multirow{3}{*}{ Group } & \multicolumn{3}{|c|}{ Fruit } & \multicolumn{3}{|c|}{ Vegetable } & \multicolumn{3}{|c|}{ Fruit \& Vegetable } \\
\hline & \multicolumn{3}{|c|}{ Estimated \# of Servings } & \multicolumn{3}{|c|}{ Estimated \# of Servings } & \multicolumn{3}{|c|}{ Estimated \# of Servings } \\
\hline & Baseline* & Post** & $\mathbf{p}$ & Baseline* & Post** & p & Baseline* & Post** & p \\
\hline $\begin{array}{l}\text { Intervention } \\
(n=16)\end{array}$ & \multirow{3}{*}{$1.01 \pm 0.54$} & \multirow[b]{2}{*}{$1.30 \pm 0.93$} & \multirow{3}{*}{0.13} & \multirow[b]{2}{*}{$0.33 \pm 0.60$} & \multirow[b]{2}{*}{$0.03 \pm 0.13$} & \multirow{3}{*}{0.06} & & \multirow[b]{2}{*}{$1.33 \pm 0.97$} & \multirow{3}{*}{0.95} \\
\hline $\begin{array}{r}\text { Number, } \\
\text { mean } \pm \text { SD }\end{array}$ & & & & & & & $1.34 \pm 0.97$ & & \\
\hline (range) & & $(0-3)$ & & $(0-2)$ & $(0-1)$ & & $(0-4)$ & $(0-3)$ & \\
\hline Control $(n=13)$ & \multirow{3}{*}{$1.11 \pm 0.46$} & \multirow{3}{*}{$1.02 \pm 0.65$} & \multirow{3}{*}{0.45} & \multirow{3}{*}{$0.21 \pm 0.31$} & \multirow{3}{*}{$0.08 \pm 0.28$} & \multirow{3}{*}{0.19} & \multirow{3}{*}{$1.26 \pm 0.47$} & \multirow{3}{*}{$\begin{array}{c}1.10 \pm 0.76 \\
(0-2)\end{array}$} & \multirow{3}{*}{0.20} \\
\hline $\begin{array}{r}\text { Number, } \\
\text { mean } \pm \text { SD }\end{array}$ & & & & & & & & & \\
\hline (range) & & & & & & & & & \\
\hline
\end{tabular}

*3-day average before intervention

started

**- day during the week post study 
Post study observations of vegetable servings were lower when compared to those at baseline in both groups; $0.03 \pm 0.12$ servings in the intervention group $(\mathrm{p}=0.06)$ and 0.08 \pm 0.28 servings the control group. When the observations of fruit and vegetable servings were combined, the number of $\mathrm{F} / \mathrm{V}$ servings was similar at baseline and post study for both groups. Observations for $\mathrm{F} / \mathrm{V}$ were $1.34 \pm 0.97$ servings at baseline and $1.33 \pm 0.97$ servings post study in the intervention group and $1.26 \pm 0.47$ servings at baseline and 1.10 servings \pm 0.76 servings post study in the control group. A one-way ANOVA and paired t-test were completed with the combined F/V data and the groups did not differ significantly at baseline or post study.

The consumption of the F/V was estimated at the end of the lunch period and recorded as the percent of the servings in the packed lunch observed and recorded prior to the lunch period; consumption is presented as number of servings in Figure 7. There were no differences between the groups from observations obtained at baseline or post study for either packed $\mathrm{F} / \mathrm{V}$ or consumption of $\mathrm{F} / \mathrm{V}$. At baseline the intervention group had $1.34 \pm 0.97$ of packed F/V servings, with an estimated consumption of $1.14 \pm 0.64$ servings. The control group had $1.26 \pm 0.47$ packed F/V servings with an estimated consumption of $1.14 \pm 0.51$ servings. Post study, the intervention group had $1.33 \pm 0.97$ of packed F/V servings with an estimated consumption amount of $1.05 \pm 0.83$ servings and the control group had $1.10 \pm 0.76$ of packed $\mathrm{F} / \mathrm{V}$ servings with an estimated consumption of $0.82 \pm 0.78$. Although the estimated amount consumed is lower in the control group at the post study observation, this difference is not significant $(\mathrm{p}=0.16)$. 
Figure 7: The amount of fruit and vegetable (F/V) servings packed in school lunches compared to the estimated consumption at baseline and post study in middle school children included in an eight-week multimedia educational program focused on the importance of $\mathrm{F} / \mathrm{V}$ intakes. 


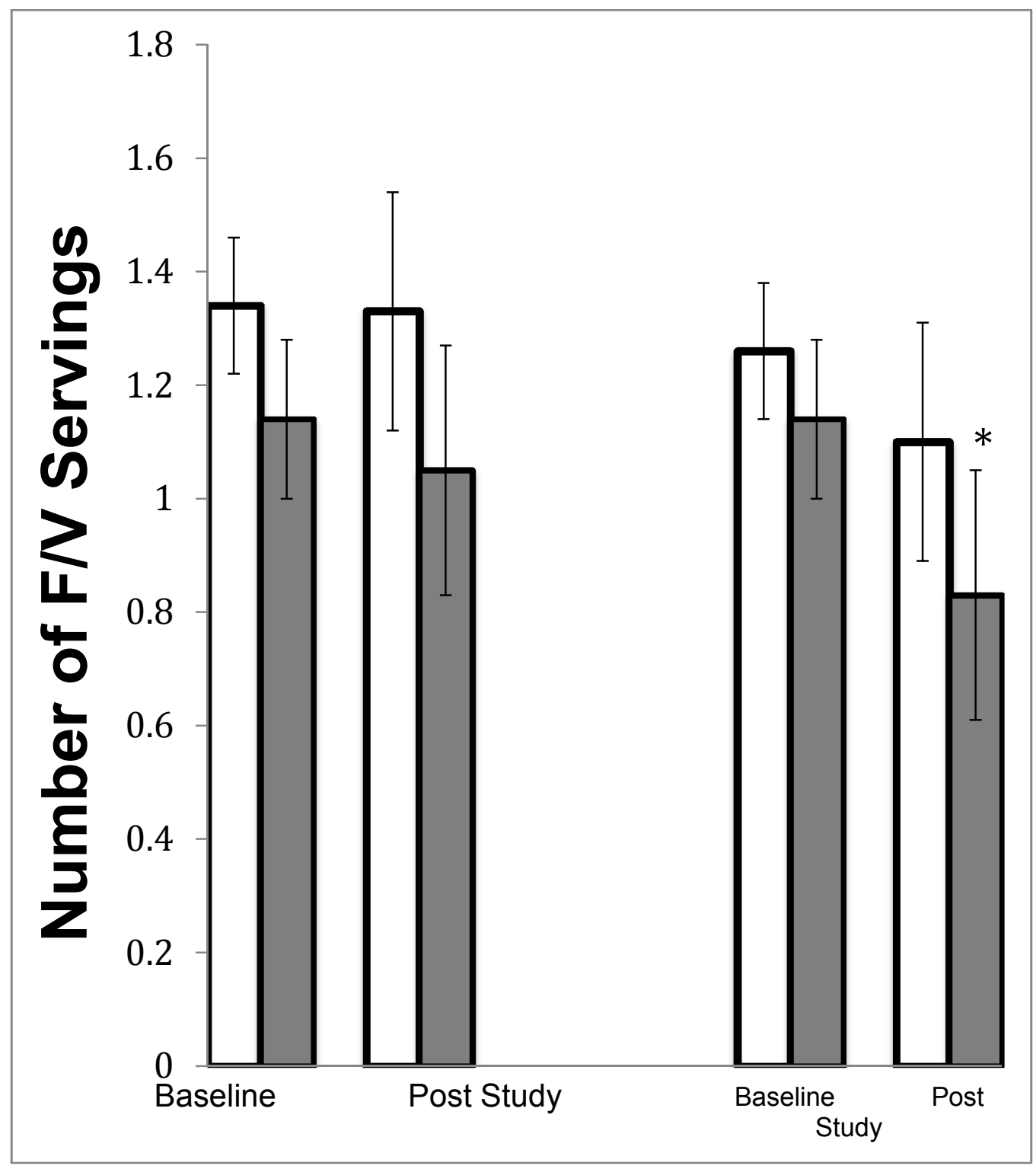

*SEM=standard error of the mean $* * \mathrm{p}=0.16$

Packed F/V servings (mean $\pm \mathrm{SEM}^{*}$ )

Estimated Consumption of F/V servings (mean \pm SEM) 
A Pearson product correlation was computed to observe the relationship between packed F/V and estimated consumption with data combined for both groups see Figure 8 . At baseline, there was somewhat of a relationship, $r$-value $=0.35(\mathrm{p}=0.06)$. Post study, the relationship between packed F/V servings and estimated consumption servings increased significantly, $\mathrm{r}$-value $=0.66(\mathrm{p}=<0.01)$. This suggests students in either group, for the most part, ate the F/V servings that were packed in the lunches and their tendency to do so was significantly greater at the post study observation.

The types of fruits and vegetables that were most often present in the packed school lunches were recorded. In both groups, apples or applesauce was most frequently observed in the fruit category, followed by mandarin oranges, and grapes. The most common vegetable was carrot, followed by sweet peppers, and salad. These did not differ in either group or at either time period.

Attitude and Self-Efficacy/Perception: The results of the attitude and selfefficacy/perception scores derived from the Farm to Table survey are presented in Table 11. No statistically significant differences in mean attitude scores were noted between groups at baseline or post study (ANOVA, $\mathrm{p}=0.217$ ); however, the two groups were somewhat different at baseline. The intervention group had a lower initial mean $\pm \mathrm{SD}$ score of $61.92 \pm 7.76$ compared to the control group $68.38 \pm 11.02(\mathrm{p}=0.089)$; both were within the normal expected range of scores. In the intervention group, the attitude scores increased by three percent from baseline to post study; however, no change was observed in the control group. 
Figure 8: Pearson product correlation to determine the relationship between $\mathrm{F} / \mathrm{V}$ present in the meal and the consumption of $\mathrm{F} / \mathrm{V}$ in both groups. At baseline the presence of $\mathrm{F} / \mathrm{V}$ and consumption was somewhat related with an $r$-value $=(p=0.06)$. Post study the relationship increased with an $\mathrm{r}$-value of $(\mathrm{p}=\leq 0.01)$, suggesting students were very likely to consume the $\mathrm{F} / \mathrm{V}$ foods that were present in the meal. 
A. Baseline comparison of both groups of foods present in the meal and the consumption.

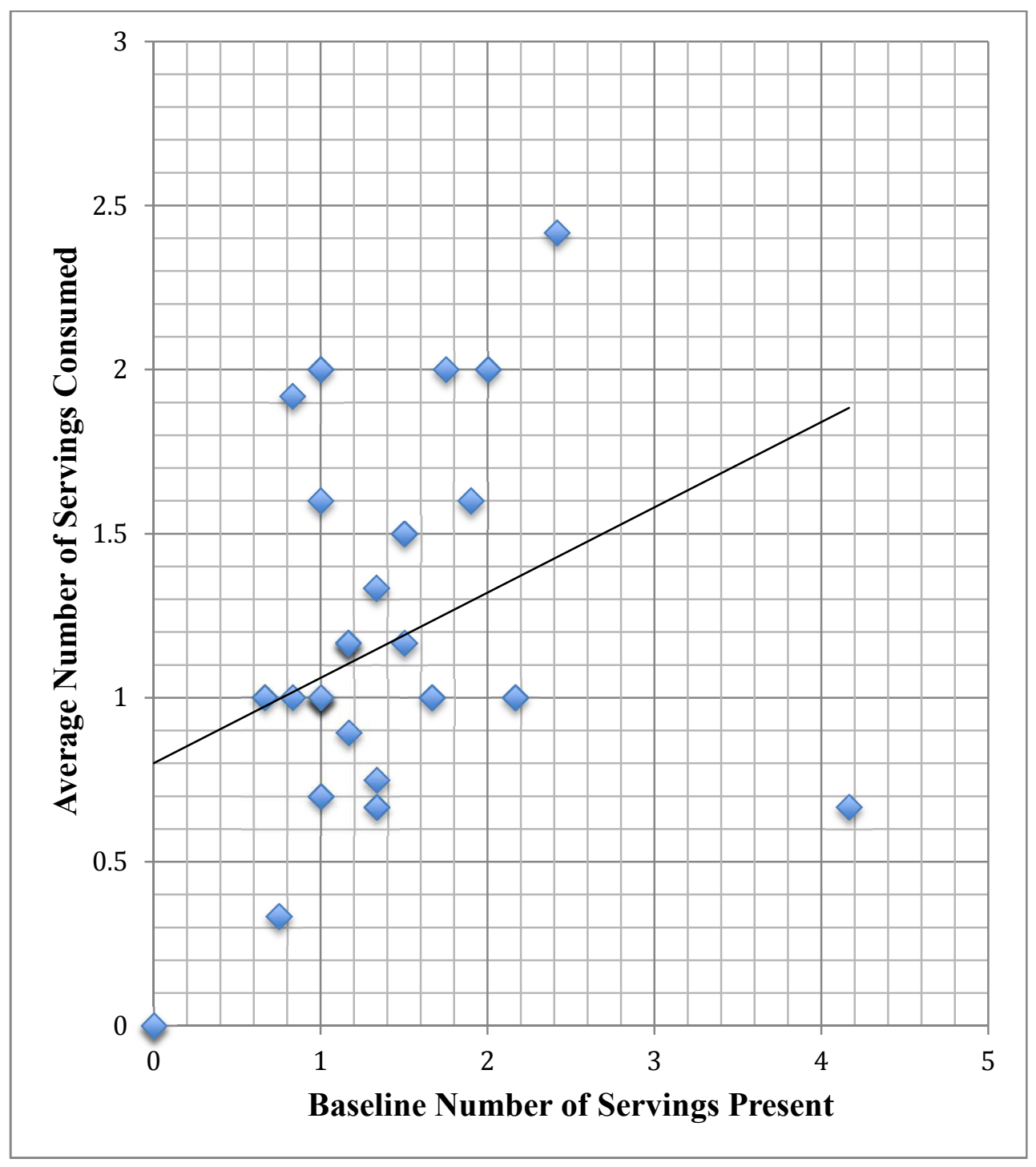


B. Post study comparison of $\mathrm{F} / \mathrm{V}$ present in the meal and consumption.

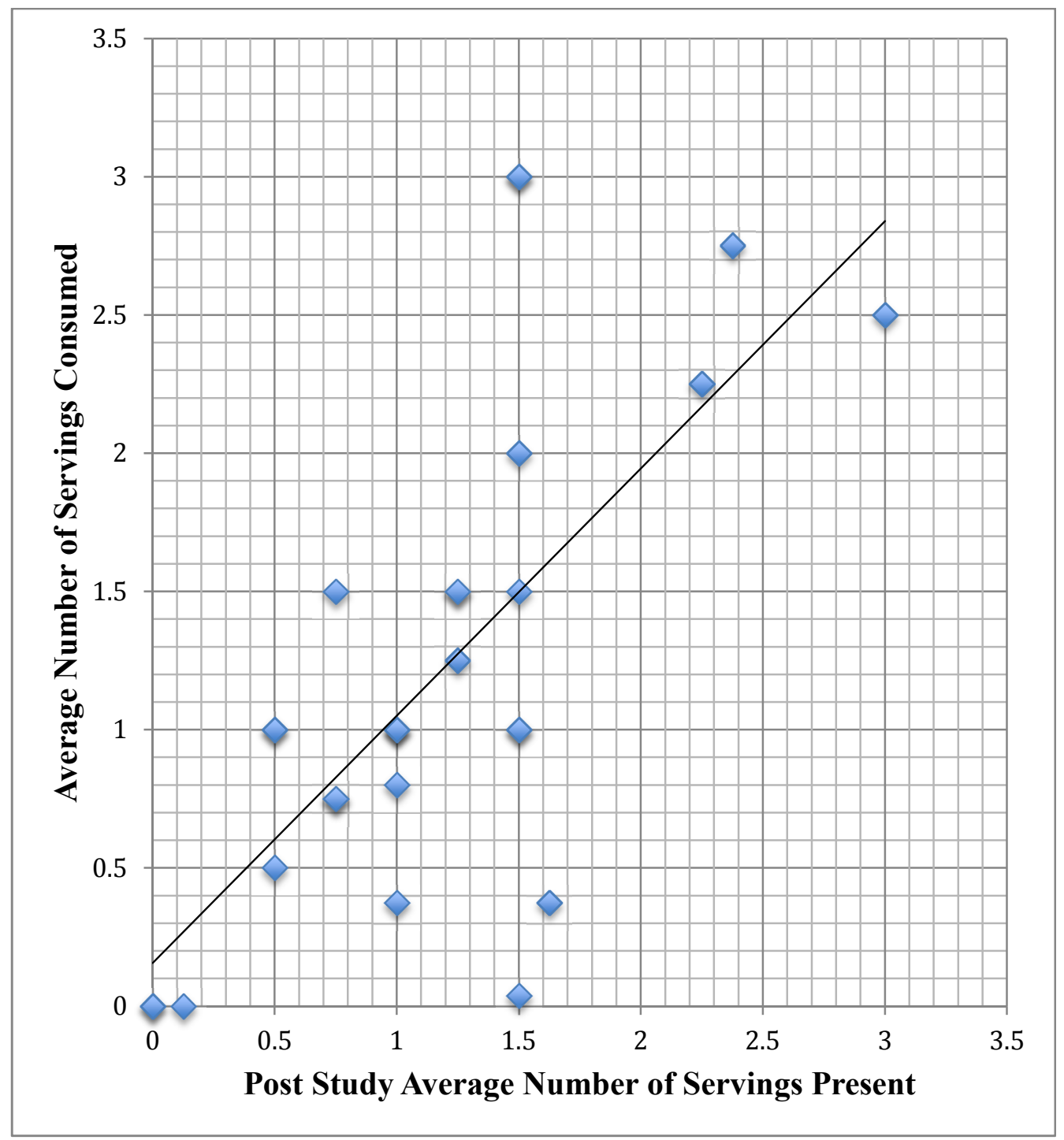


Table 11: Attitude and self-efficacy/perception scores derived from the Farm to Table* assessment survey given to middle school children enrolled in an eight-week multimedia educational program focused on the importance of fruit and vegetable intakes 


\begin{tabular}{|c|c|c|c|c|c|c|}
\hline \multirow[t]{2}{*}{ Group } & \multicolumn{3}{|c|}{$\begin{array}{l}\text { Attitude } \\
\text { Scores }\end{array}$} & \multicolumn{3}{|c|}{$\begin{array}{c}\text { Self-Efficacy/Perception } \\
\text { Scores }\end{array}$} \\
\hline & Baseline & Post & $\mathbf{p}$ & Baseline & Post & p \\
\hline \multicolumn{7}{|l|}{ Intervention $(n=16)$} \\
\hline $\begin{array}{r}\text { Score, mean } \pm \text { SD } \\
\text { (range) } \\
\text { Number of children surveyed }\end{array}$ & $\begin{array}{c}61.92 \pm 7.76^{* * *} \\
(50-76) \\
14\end{array}$ & $\begin{array}{c}63.71 \pm 7.17 \\
(49-79) \\
16\end{array}$ & 0.29 & $\begin{array}{c}25.07 \pm 3.07 \\
(19-29) \\
14\end{array}$ & $\begin{array}{c}25.92 \pm 2.30 \\
(19-29) \\
16\end{array}$ & 0.29 \\
\hline \multicolumn{7}{|l|}{ Control $(n=13)$} \\
\hline $\begin{array}{r}\text { Number, mean } \pm \text { SD } \\
\text { (range) } \\
\text { Number of children surveyed }\end{array}$ & $\begin{array}{c}68.38 \pm 11.02^{* *} \\
(43-85) \\
13\end{array}$ & $\begin{array}{c}68.00 \pm 9.26 \\
(49-84) \\
13\end{array}$ & 0.86 & $\begin{array}{c}25.69 \pm 2.98 \\
(20-30) \\
13\end{array}$ & $\begin{array}{c}25.38 \pm 2.72 \\
(20-28) \\
13\end{array}$ & 0.64 \\
\hline
\end{tabular}

*Farm to Table Survey ("Tool: Knowledge, attitude, and consumption behavior survey," 2010)

$* * \mathrm{p}=0.089$ 
Mean scores for perception/self-efficacy were not different between groups, and they did not change baseline to post study in either group. The intervention group had a mean \pm SD baseline score of $25.07 \pm 3.07$ and post study score of $25.92 \pm 2.30$. The control group had a mean baseline score of $25.92( \pm 2.30)$ and post study score of 25.38 $( \pm 2.72)$. No differences were noted in either group or between groups at baseline and post study.

\section{Knowledge Scores}

Knowledge scores, derived from the adapted Farm to Table survey, significantly improved from baseline to post study in the intervention group (ANOVA, $\mathrm{p}=0.001$ ); however, no improvement was observed in the control group. At baseline, both groups had similar scores out of 11 questions; the intervention group had a mean of 8.00 correct and 7.38 questions were correct in the control group compared to post study means of 10.00 correct questions in the intervention group and 7.78 in the control group. The intervention group improved their scores with a 20 percent increase in knowledge, whereas the control group demonstrated no change in scores; this data is shown in Figure 8. The questions that appeared to have the most improvement included: (1) What is the role of fiber in food? (2) How would you best describe the word "microbiota" ? (3) What helps improve the health of your gut or your intestines? (4) Which of these is the healthiest form of potatoes?

\section{Fruit and Vegetable (F/V) Screener and Relative Amounts}

The results of the 24-hour F/V screener component of the Farm to Table survey are presented in Table 12 as percentages of students who indicated that they included F/V 
Figure 9: Knowledge scores (mean $\pm \mathrm{SEM}$ ), at baseline and post study, obtained from the adapted Farm to Table in middle school children enrolled in an eight-week multimedia educational program focused on the importance of fruit and vegetable intakes. 


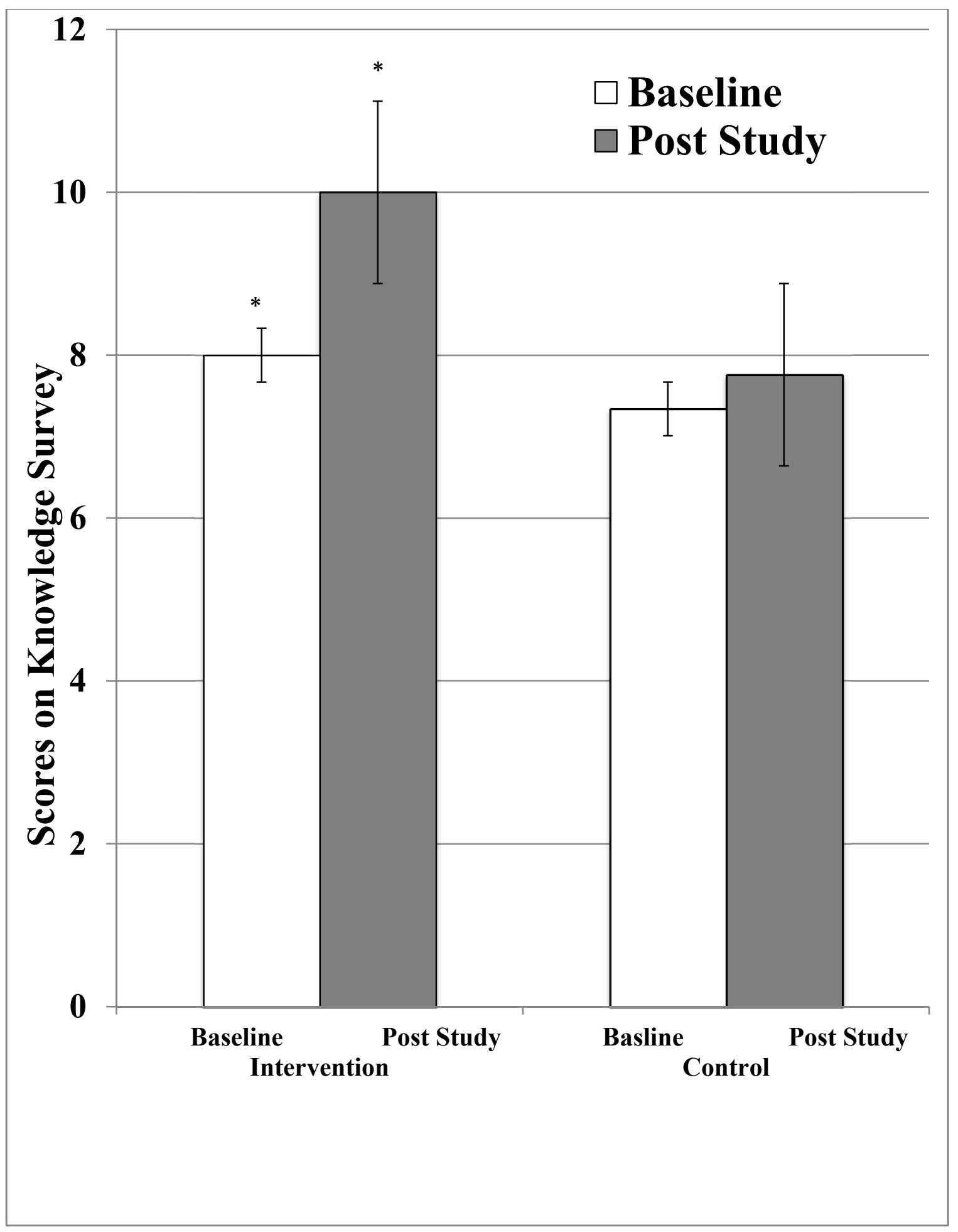

$* p=0.001$ 
in their diets as well as the relative amounts of F/V. In some instances, students indicated that they did not include a food in their diet, however, also indicated that an amount that was eaten of the same food item that was reported as not included. The data was analyzed by removing these inconsistent responses.

In the intervention group, consistent data from 10 - 16 students were included in the analysis. At baseline, a range of 30-93 percent intake of foods in the four different F/V food groupings (fruit, potato, vegetable, legume) was reported. At post-study, a range of 23-100 percent intake of foods in the same groupings was reported for all categories except potatoes. The total $\mathrm{F} / \mathrm{V}$ estimated relative amount reported at baseline was $1.74 \pm 1.19$ servings and a significant increase to $2.65 \pm 1.63$ servings was reported at the post study measure $(\mathrm{p}=0.03)$. The 24 -hour relative amount for vegetables serving increased significantly from 0.60 servings \pm 0.40 servings at baseline to $0.89 \pm 0.40$ post study ( $\mathrm{p}=0.004)$; this is roughly equivalent to a 30 percent increase in the serving amount. In the control group, consistent data from 11-13 students were included in the analysis. Students in this group reported a range of 27-100 percent consumption of F/V in the previous 24 hours for all four categories of F/V. Post-study, the reported range was 31-100 percent in all F/V groupings. The control group reported a relative amount of total $\mathrm{F} / \mathrm{V}$ intake of $1.70 \pm 1.19$ servings at baseline and $2.28 \pm 1.34$ servings post study $(\mathrm{p}=0.16)$. The 24-hour relative amount for fruit servings increased significantly from 0.72 \pm 0.33 servings at baseline to $0.94 \pm 0.36$ servings post study ( $\mathrm{p}=0.01$ ); this is roughly equivalent to a 31 percent increase in the serving amount. 
Table 12: Percentages and relative amounts (mean $\pm \mathrm{SD}$ ) of fruit, potato, vegetable, and legumes using the 24-hour Farm to Table Survey at baseline and post study in middle school children included in an eight-week multimedia educational program focused on the importance of fruit and vegetable intakes. 


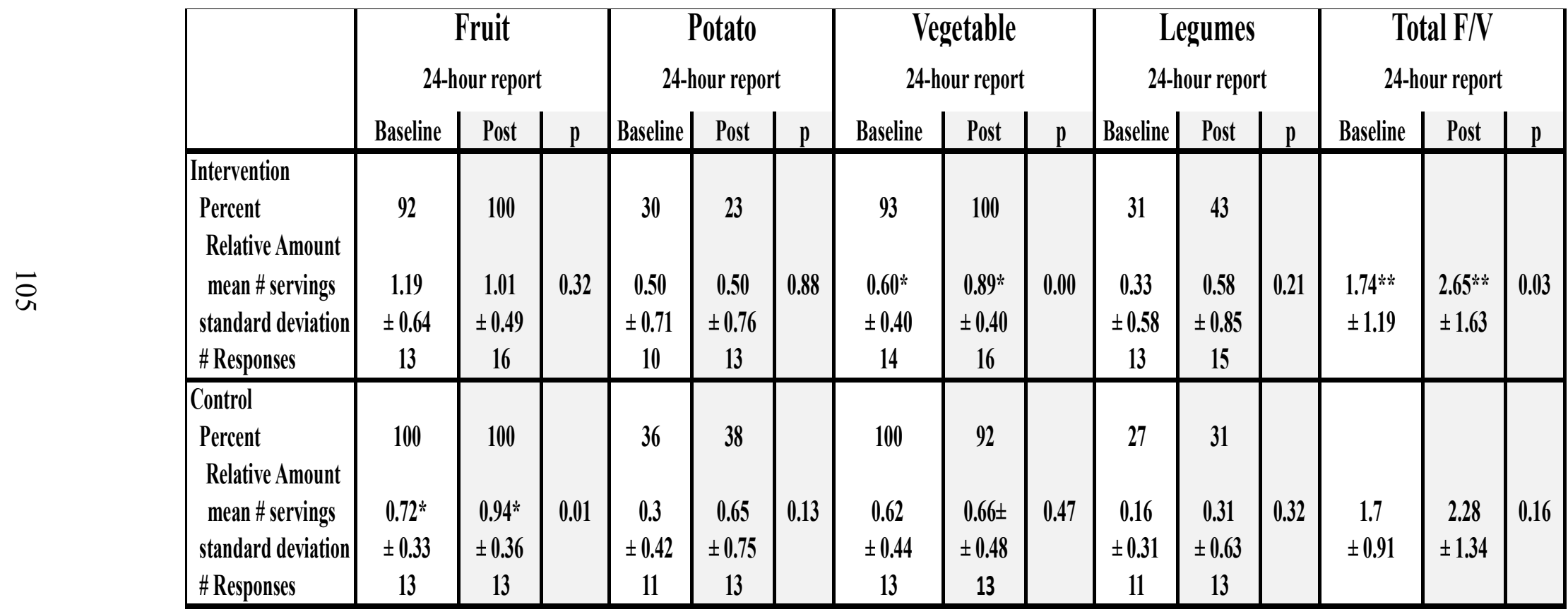

" $\mathrm{p} \leq 0.01$

${ }^{*} \mathrm{k} p<0.05$ 


\section{Day in the Life Questionnaire (DILQ):}

The results from the DILQ are presented in Table 13. The mean F/V servings reported by students in writing and/or picture form during the time frame that included breakfast through the after-school snack were higher in both groups at baseline compared to post study. The intervention group reported $2.63( \pm 1.85 \mathrm{sd})$ servings at baseline and $1.13( \pm 1.12 \mathrm{sd})$ servings post study $(\mathrm{p}=0.07)$. The control group reported $2.17( \pm 1.40 \mathrm{sd})$ servings at baseline and $1.58( \pm 1.16 \mathrm{sd})$ servings post study $(\mathrm{p}=0.31)$. Post study reports that captured intakes from the entire day, breakfast through evening snack reflected mean daily servings higher in the control group with $2.75( \pm 1.75 \mathrm{sd})$ servings compared to 2.11 $( \pm 1.69$ SD) servings in the intervention group $(p=0.30)$.

\section{Parent Reports of F/V Food Offered to and Consumed by Their Child from the Eating at America's Table Survey (EATS) Survey:}

The number (mean $\pm \mathrm{SD}$ ) of $\mathrm{F} / \mathrm{V}$ servings reported by parents, at baseline, for each food and/or category is presented in Figure 9 for the intervention and control groups. Fruit in units is the highest reported food item offered to and consumed by the children with $1.36 \pm 1.30$ servings daily in the intervention group and $1.67 \pm 1.54$ servings in the control group. The least frequently reported food group is French fries in both groups $0.03 \pm 0.04$ servings in the intervention group and $0.02 \pm 0.03$ servings in the control group. The total mean servings reported by parents of all food categories offered to and consumed by their children was $3.88 \pm 3.64$ servings daily in the intervention group and $3.24 \pm 1.98$ servings in the control group; The reports were not significantly different between groups $(\mathrm{p}=0.63)$. 
Table 13: The number of F/V servings reported in the Day in the Life Questionnaire (DILQ) from breakfast through the after-school snack at baseline and post study and breakfast through after dinner snack at post study in middle school children enrolled in an eight-week multimedia educational program focused on the importance of fruit and vegetable intakes. 


\begin{tabular}{|l|c|c|c|c|}
\hline & \multicolumn{3}{|c|}{ Breakfast to After School Snack } & $\begin{array}{c}\text { Breakfast to } \\
\text { After Dinner } \\
\text { Snack }\end{array}$ \\
\hline & Baseline & Post Study & p & Post Study \\
\hline Intervention Group & & & & \\
\hline F/V Servings (mean \pm SD) & $2.62 \pm 1.85$ & $1.13 \pm 1.13$ & 0.07 & $2.11 \pm 1.69^{*}$ \\
\hline Number of responses (n) & 14 & 9 & & \\
\hline Control Group & & & & \\
\hline F/V Servings (mean \pm SD) & $2.17 \pm 1.40$ & $1.58 \pm 1.16$ & 0.31 & $2.75 \pm 1.05^{*}$ \\
\hline Number of responses (n) & 13 & 12 & & 12 \\
\hline
\end{tabular}

* $p=0.30$ 
Figure 10: Results of the Eating at America's Table Survey (EATS): Parent reported number of F/V servings offered to and consumed by their middle school children enrolled in an eight-week multimedia educational program focused on the importance of fruit and vegetable intakes. 


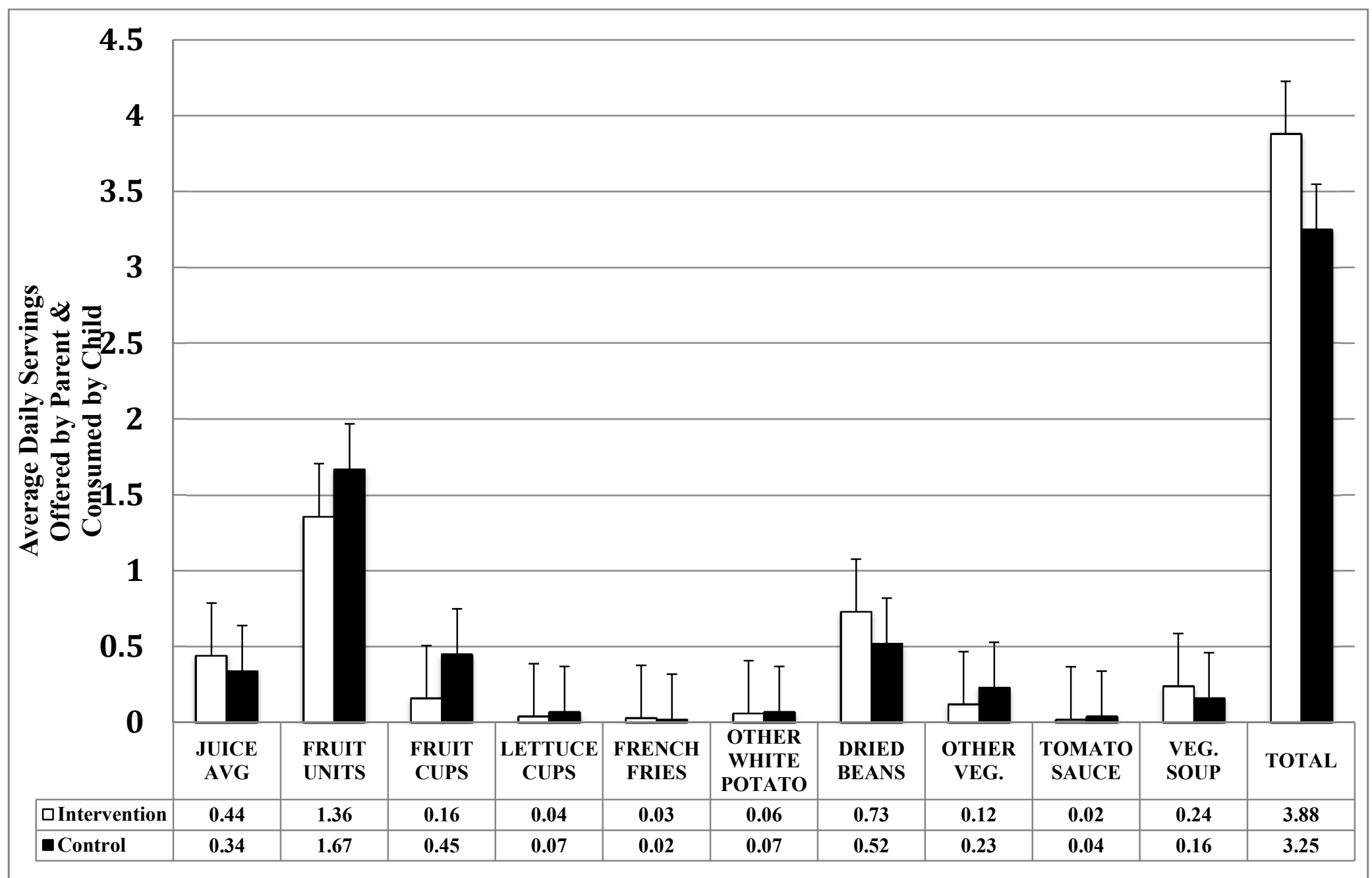

Categories of Fruits and Vegetables (F/V) From the EATS Survey 


\section{Parent Reports of F/V Servings from the Adapted Youth Adolescent Food Frequency Questionnaire (YAQ); Weekly/Monthly}

The YAQ weekly assessment results are presented in Figure 10. Parents in both groups reported that their children consumed the most servings from the vegetable group (not including potatoes and beans) with mean \pm SD intakes of $1.25 \pm 0.21$ servings in the intervention group and $1.18 \pm 0.45$ servings in the control group. Reported intakes from the fruit group were the next highest category in prevalence, $0.98 \pm 0.17$ servings in the intervention group and $1.02 \pm 0.41$ servings in the control group; when data was combined for both the intervention and control groups, the mean reported intake was 1.20 \pm 0.38 servings per day in both the intervention and control groups. The total amount of all $\mathrm{F} / \mathrm{V}$ categories, with the exclusion of the fiber/grain group, was $3.16 \pm 0.43$ servings in the intervention group and $2.71 \pm 0.45$ servings in the control. When data from both groups was combined for all food categories, with the exclusion of the fiber/grain group, the estimated mean $\pm \mathrm{SD}$ daily $\mathrm{F} / \mathrm{V}$ intake was $2.94 \pm 0.48$ servings.

When parents recalled intakes over a month's time frame, SIMILAR estimated food category servings were noted; these are presented in Figure 11. Over the month time frame the fruit category was the highest reported grouping by parents with $0.34 \pm 0.40$ servings in the intervention group and $0.61 \pm 0.32$ servings in the control group (Figure 12). When data from both groups was combined for all food categories, with the exclusion of the fiber /grain group, the estimated mean $\pm \mathrm{SD}$ daily F/V intake was $1.31 \pm$ 0.75 servings. 
Figure 11: Results of the adapted Youth Adapted Food Frequency Questionnaire (YAQ): Parent weekly recalls of the number of servings within groups of $\mathrm{F} / \mathrm{V}$ for their middle school children enrolled in an eight-week multimedia educational program focused on the importance of fruit and vegetable intakes. 


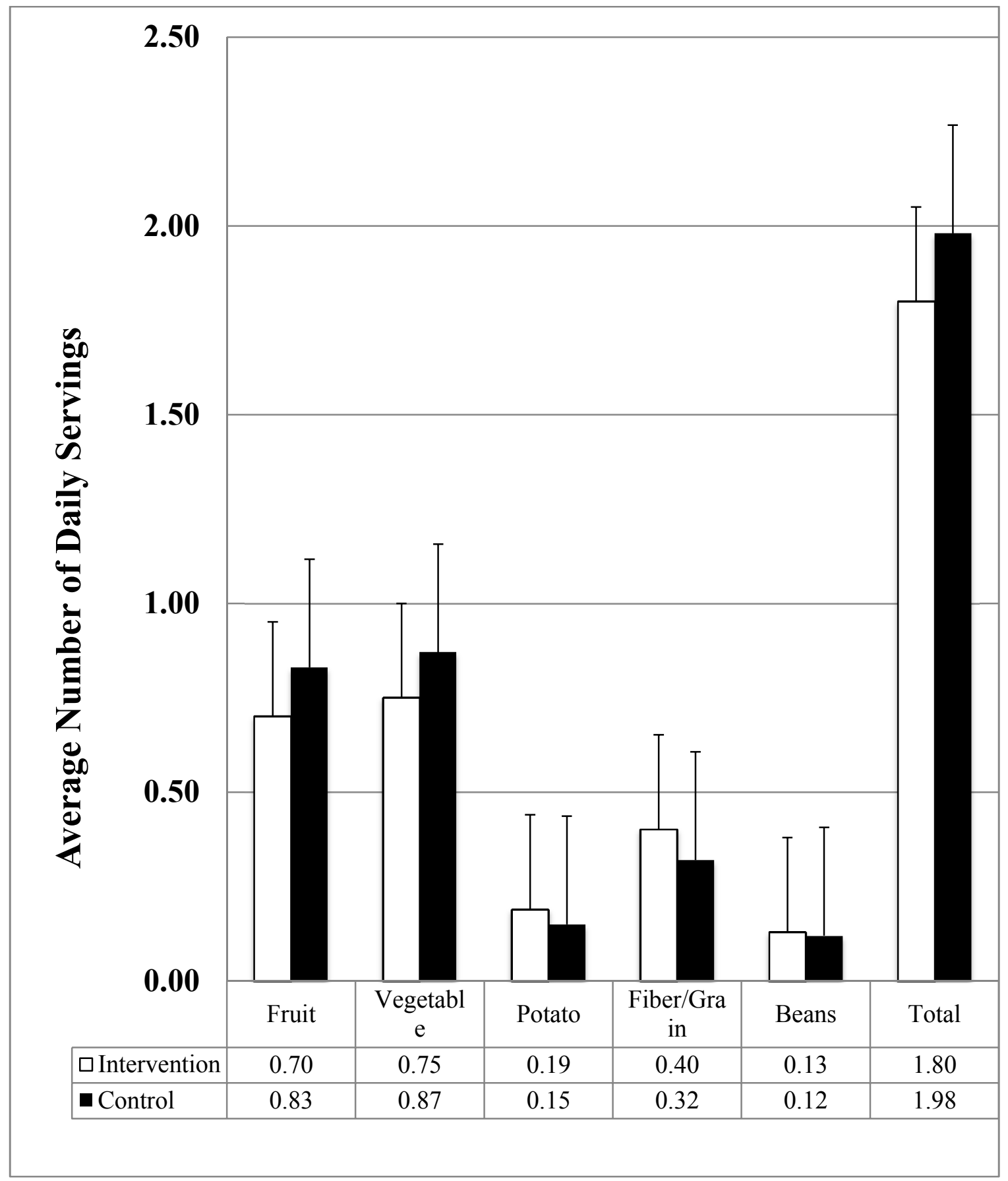

Categories of Fruits and Vegetables (F/V) From the YAQ 
Figure 12: Results of the adapted Youth Adapted Food Frequency Questionnaire (YAQ): Parent monthly recalls of the number of servings within groups of $\mathrm{F} / \mathrm{V}$ for their middle school children enrolled in an eight-week multimedia educational program focused on the importance of fruit and vegetable intakes. 


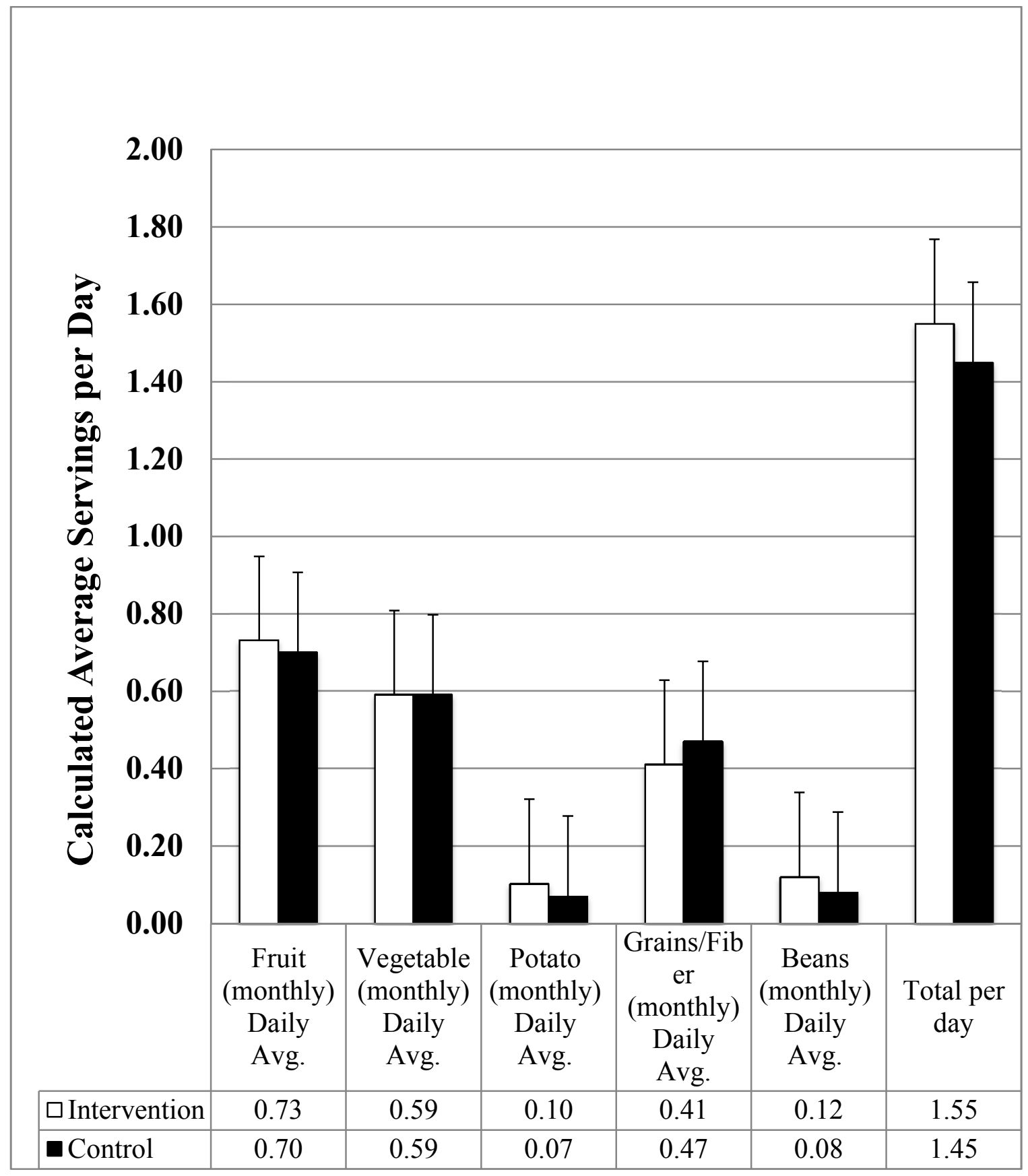

Categories of Fruits and Vegetables (F/V) From the YAQ 
Aim 2: To determine if personal factors such as BMI, blood pressure, and gender relate to the amounts and types of F/V included in and consumed from home-prepared school lunches among middle school children ages 11-12 years.

Measurements of height, weight and blood pressure and calculated values of BMI and BMI z-scores (mean $\pm \mathrm{SD}$ ), at baseline and post study time periods, are presented in Table 14. No differences were observed between the groups for anthropometric measures or blood pressure at baseline or post study. The mean baseline height in both groups was 155.12 centimeter $(\mathrm{cm})$ and the mean baseline weight was 47 kilograms $(\mathrm{kg})$. The mean baseline BMI recorded was $19.46 \mathrm{ht} / \mathrm{m}^{2}$ in both groups. The mean baseline systolic/diastolic blood pressures were 102.37/63.48 mm Hg. Parent's age, BMI, and blood pressure measurements were not recorded. Before the intervention period and at the end of the study, height, weight, BMI, and blood pressure measurements were recorded. No significant changes were found between or within groups for any of the listed measures based on one-way ANOVA. No clinically significant changes were noted at baseline and post intervention.

The effects of gender, BMI (z-scores) and blood pressure on the presence of F/V in packed school lunches were assessed. Independent T-Tests were used to measure the impact of these independent variables on the dependent variable (any $\mathrm{F} / \mathrm{V}$ servings in the packed school lunch): yes or no. There were no significant relationships identified at baseline, however a significant difference at post study was noted for gender; 0.026 (95\% Confidence Interval of -1.21 and -0.79 ). This was likely due to more girls having any amount of $\mathrm{F} / \mathrm{V}$ in their packed lunches compared to three boys who did not have any $\mathrm{F} / \mathrm{V}$ in their packed lunches post study, but did have them at baseline. 
Table 14: Baseline and post study measures of a) height and weight; b) calculated BMI and BMI z-scores; and c) blood pressure (systolic and diastolic) of middle school children enrolled in an eight-week multimedia educational program focused on the importance of fruit and vegetable intakes. 


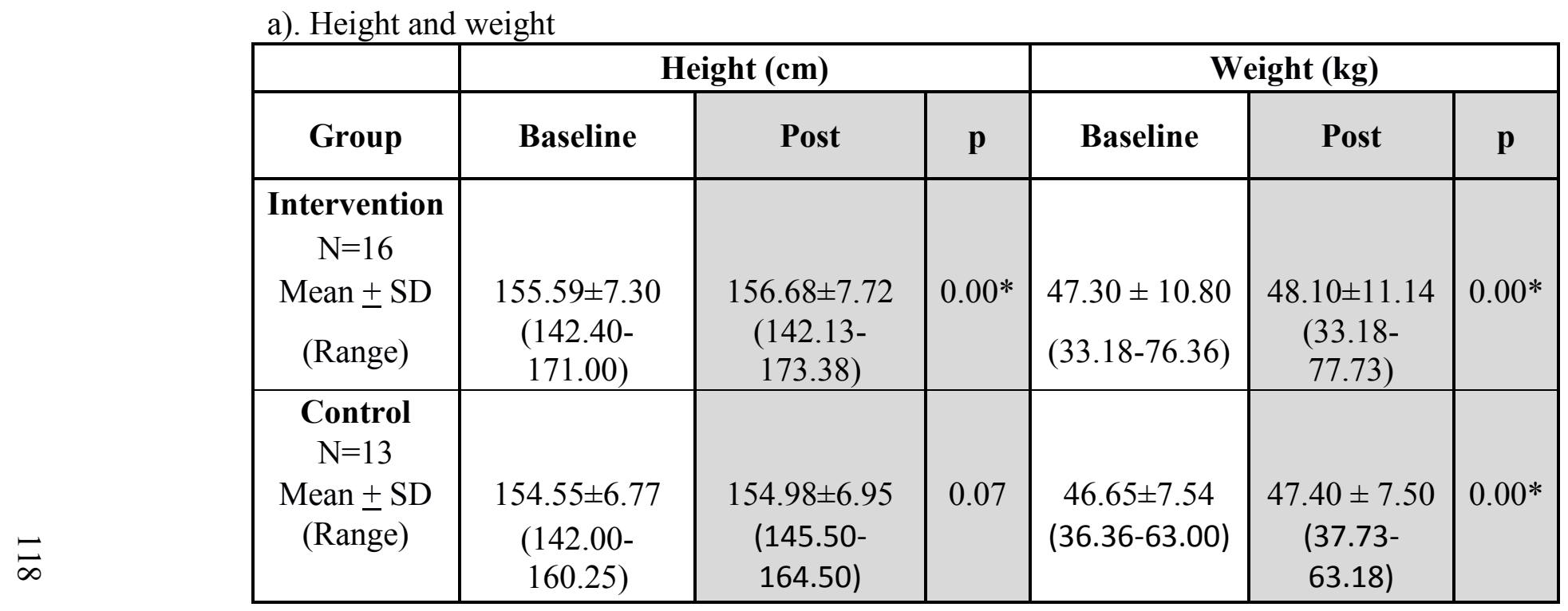

*not clinically significant

b). BMI

\begin{tabular}{|c|c|c|c|c|c|c|}
\hline \multirow[b]{2}{*}{ Group } & \multicolumn{3}{|c|}{ BMI $\left(\mathrm{Ht} / \mathrm{m}^{2}\right)$} & \multicolumn{3}{|c|}{ BMI Z-Score } \\
\hline & Baseline & Post & $\mathbf{p}$ & Baseline & Post & $\mathbf{p}$ \\
\hline $\begin{array}{c}\text { Intervention } \\
\mathrm{N}=16\end{array}$ & & & & & & \\
\hline $\begin{array}{c}\text { Mean } \pm \text { SD } \\
\text { (Range) }\end{array}$ & $\begin{array}{c}19.40 \pm 3.38 \\
(15.98-26.11) \\
\end{array}$ & $\begin{array}{c}19.49 \pm 3.26 \\
(16.24-25.97) \\
\end{array}$ & 0.13 & $\begin{array}{l}0.37 \pm 0.95 \\
(-0.50-2.00)\end{array}$ & $\begin{array}{c}0.53 \pm 0.84 \\
(-0.50-2.00)\end{array}$ & $0.05^{*}$ \\
\hline $\begin{array}{c}\text { Control } \\
\mathrm{N}=13\end{array}$ & & & & & & \\
\hline $\begin{array}{c}\text { Mean } \pm \text { SD } \\
\text { (Range) }\end{array}$ & $\begin{array}{c}19.55 \pm 3.07 \\
(15.63-26.27)\end{array}$ & $\begin{array}{c}19.80 \pm 3.00 \\
(15.53-25.96)\end{array}$ & $0.05 *$ & $\begin{array}{c}0.35 \pm 0.94 \\
(-0.5-2.00)\end{array}$ & $\begin{array}{c}0.61 \pm 0.84 \\
(-0.50-2.00)\end{array}$ & $0.00 *$ \\
\hline
\end{tabular}

*not clinically significant 
c). Blood pressure

\begin{tabular}{|c|c|c|c|c|c|c|}
\hline \multirow[b]{2}{*}{ Group } & \multicolumn{3}{|c|}{ Systolic Blood Pressure (mm) } & \multicolumn{3}{|c|}{ Diastolic Blood Pressure (mm) } \\
\hline & Baseline & Post & $\mathbf{p}$ & Baseline & Post & $\mathbf{p}$ \\
\hline $\begin{array}{c}\text { Intervention } \\
\mathrm{N}=16\end{array}$ & & & & & & \\
\hline $\begin{array}{c}\text { Mean } \pm \text { SD } \\
\text { (Range) }\end{array}$ & $\begin{array}{c}104.25 \pm 9.40 \\
(86-119)\end{array}$ & $\begin{array}{c}99.37 \pm 11.90 \\
(79-115)\end{array}$ & $0.03 *$ & $\begin{array}{l}62.62 \pm 8.08 \\
\quad(51-79)\end{array}$ & $\begin{array}{c}64.88 \pm 10.33 \\
(50-88)\end{array}$ & 0.49 \\
\hline $\begin{array}{c}\text { Control } \\
\mathrm{N}=13\end{array}$ & & & & & & \\
\hline $\begin{array}{c}\text { Mean } \pm \text { SD } \\
\text { (Range) }\end{array}$ & $\begin{array}{c}100.07 \pm 8.85 \\
(81-118)\end{array}$ & $\begin{array}{c}98.00 \pm 7.49 \\
(83-111)\end{array}$ & 0.37 & $\begin{array}{c}64.54 \pm 5.53 \\
(53-71)\end{array}$ & $\begin{array}{c}62.46 \pm 9.40 \\
(47-81)\end{array}$ & 0.55 \\
\hline
\end{tabular}

*not clinically significant 


\section{Parental Involvement}

Parents were involved in three surveys: EATS, YAQ weekly recall, and YAQ monthly recall and those in the intervention group were also asked to create a grocery shopping list with their child. Only 10 parents in the intervention group returned signed forms and six did not return them, even after reminders. An Independent t-test was conducted to determine if differences exist between the involvement of parents (yes or no) and the amount of F/V servings observed in packed lunches post intervention as well as the amount consumed. No statistically significant relationships between parental involvement in making a grocery store list and presence of $\mathrm{F} / \mathrm{V}$ in the lunch or consumption of $\mathrm{F} / \mathrm{V}$ were identified $(\mathrm{p}=0.45)$.

\section{Relationship Among Assessment Tools}

Estimates of $\mathrm{F} / \mathrm{V}$ servings were obtained independently from nine assessments. Two completed by children at baseline and repeated post study, three completed by parents at baseline, and one observation completed by the research team at baseline and repeated post study. A significant correlation was observed between the $\mathrm{F} / \mathrm{V}$ school lunch observations and the YAQ monthly survey at baseline $\mathrm{p}=0.003$. A significant correlations was observed between the EATS survey and the YAQ monthly at baseline $p=0.024$. This suggests relationships exist between these research methods and tools. Post study, the means for the two surveys for relative amount and DILQ were very similar and likely would show a significant relationship with a larger participant population. The means for estimated F/V daily servings are presented in Table 15 for baseline and post study results. 
Table 15: Comparison of the estimated number of fruit and vegetable (F/V) servings (mean $\pm \mathrm{SD}$ ) determined from all surveys at baseline and post study in middle school children enrolled in an eight-week multimedia educational program focused on the importance of fruit and vegetable intakes. 


\begin{tabular}{|c|c|c|c|}
\hline $\begin{array}{c}\text { Statistical } \\
\text { Correlation }\end{array}$ & $\begin{array}{c}\text { F/V Servings in } \\
\text { Packed School } \\
\text { Lunch }\end{array}$ & Comparison Survey & $\mathbf{p}$ \\
\hline Baseline & & & \\
\hline $\begin{array}{c}\text { F/V school lunch } \\
\text { observations }\end{array}$ & $1.30 \pm 0.77$ & YAQ Monthly & $0.003^{* *}$ \\
\hline Farm to Table* & $1.72 \pm 1.06$ & EATS & 0.55 \\
Relative Amounts & $3.59 \pm 2.96$ & YAQ Monthly & $0.024^{* * *}$ \\
\hline EATS* & $2.94 \pm 0.48$ & Relative Amounts & 0.67 \\
\hline YAQ Weekly* & & Farm to Table & \\
\hline DILQ* & $2.48 \pm 1.36$ & Relative Amounts & 0.5 \\
\hline YAQ Monthly* & $1.32 \pm 0.76$ & YAQ Weekly & 0.6 \\
\hline Post Study & & & 0.5 \\
\hline Farm to Table & $2.48 \pm 1.49$ & DILQ (post) & \\
\hline & & & \\
\hline
\end{tabular}

*Survey 1 = Farm to Table completed by students at baseline and post study Survey 2 = Day in the Life Questionnaire (DILQ) completed by students post study Survey 3 = Eat at America's Table (EATS) completed by parents at baseline

Survey 4 = Youth Adapted Food Frequency Questionnaire (YAQ) completed by parents at baseline $* * p<0.01$

$* * * p<0.05$ 


\section{CHAPTER V}

\section{DISCUSSION}

The overall aim of this study (Aim 1) was to determine if an educational intervention, focused on the importance of $\mathrm{F} / \mathrm{V}$ in the diet and administered to middleschool aged children (11-12 years) who attend a parochial Indianapolis-area school, impacts: (1) the amounts of F/V included in and consumed from home-prepared school lunches; (2) the students' attitudes toward F/V intake, (3) the students' knowledge of F/V benefits; and (4) the students' self-efficacy/perception related to eating F/V who receive either repeated educational video plus interactive feedback (intervention group) or a onetime educational video without interactive feedback (control group).

The framework and design of this study was based on the health theories of SCLT, self-efficacy, and HBM in order to better understand the research problem. These theory constructs were used in each step of the qualitative process beginning as the foundation for the questions used in the focus groups through to the analysis, by identifying themes that emerged related to theory constructs. The qualitative information collected at baseline was sequentially used to enhance the lessons. More specifically, in lesson one, standard terms were used in this initial lesson given to both groups that addressed the need for F/V and the servings sizes for each meal based on the age of the audience. The emerged themes related to perceived susceptibility and seriousness were further explained in the lessons to describe the health risks associated with obesity and heart disease so students could have a more in depth understanding of the impact of diet choices on the body, and to build on their initial baseline knowledge. The emerged themes related to benefits and barriers of eating $\mathrm{F} / \mathrm{V}$ were highlighted as general health 
benefits that were expanded on more in the lessons. For example, healthy body weight, low cholesterol levels, and heart benefits were further explained as being potential health benefits of eating $\mathrm{F} / \mathrm{V}$ regularly. Perceived barriers related to resources, environment, and family were addressed in the lessons building on the information students relayed during the focus groups. Low cost options of $\mathrm{F} / \mathrm{V}$, specific places to buy $\mathrm{F} / \mathrm{V}$ in bulk, and traditions within the family were discussed in the lessons to help the students with their perceived barriers of eating F/V. Self-efficacy emerged themes related to practicing how to prepare and cook $\mathrm{F} / \mathrm{V}$, and repeating these preparations were addressed in the lessons. Recipes were added to serve as a tool to help increase student interest in F/V and increase their confidence in selecting F/V more frequently.

The amount of F/V servings in home-prepared lunches did not differ significantly between the groups at the end of the study and the parents and students were blinded to the measurement of changes in $\mathrm{F} / \mathrm{V}$ in this project. In fact, the mean number of $\mathrm{F} / \mathrm{V}$ servings present in the school lunch was slightly lower in both groups at the end of the study, and the amounts of consumption declined from the baseline-estimated intakes compared to those estimated at the end of the study. This may be attributable to outside factors such as anticipation of summer vacation, classroom parties, and ice cream rewards that occurred when the study end evaluations were completed. One meaningful finding, however, that was evident in both groups at both observation periods (baseline and post study) was a strong relationship between the presence of F/V servings in the packed lunch and the estimated amounts consumed from the same lunch at baseline $(\mathrm{p}=0.06)$ and statistically significant post study $(\mathrm{p}=0.00)$, suggesting that children ate the $\mathrm{F} / \mathrm{V}$ servings if they were present in the packed lunch. Further, this finding suggests that at least 80 
percent or more of the time children are actually eating F/V packed in the their lunch. It is reasonable to assume then, if they packed more F/V servings, more would be consumed. This needs to be communicated to parents who may believe children simply will not eat $\mathrm{F} / \mathrm{V}$, as this finding supports this is not always the case.

Recalled daily average reports of F/V servings from both student and parents in both the intervention and control groups, reflect national reports (CDC, 2014; USDA, 2014). National data suggests that children, ages 6-19 years, only include about half of the recommended servings of fruit, and slightly more than half of the recommended vegetable servings in a day (USDA, 2014).

The measurement of $\mathrm{F} / \mathrm{V}$ intake can be complex and may cause confusion when recommendations are given. Therefore, there has been an effort made to simplify recommendations for the intakes of $\mathrm{F} / \mathrm{V}$ in the US as they relate to servings (USDA, 2010). The following was taken directly from a report by the CDC (CDC, n.d.) as stated below:

The newer Healthy People 2020 objectives are to increase the contribution of fruits and vegetables to the diets of Americans from 0.5 to 0.9 cup equivalent of fruits per 1,000 calories and 0.8 to 1.1 cup equivalent of total vegetables per 1,000 calories. Second, national recommendations for fruit and vegetable intake changed from a general 5-a-day recommendation for everyone, to recommendations based on an individual's age, sex, and physical activity level (CDC, n.d.).

More current, validated assessment tools; however, measure intakes of F/V in number of servings versus cups. Therefore, in this study, servings sizes were equal to a half cup for fruits and vegetables; one serving size of leafy greens was equal to one full cup (USDA, 2017). This allowed for better translation to the revised recommendations. 
Knowledge scores, post study, showed significant improvement of 20 percent in the intervention group after the " $\mathrm{F} \& \mathrm{~V}, 1 \bullet 2 \bullet 3$ " lessons where presented to them, whereas, scores at baseline in both groups and at post study in the control group were similar. This suggests that students who participated in the intervention lessons correctly answered questions related to the benefits of F/V post study when they answered the same questions incorrectly at baseline; those in the control group answered the same questions incorrectly at both baseline and post study. The construct of perceived benefits was addressed within this tool and supported the use of this theory as a positive foundation for changing knowledge scores within this population.

The improvement of knowledge scores observed in the intervention group was a similar outcome when compared to other studies (LaBrosse \& Albrecht, 2013). This is an important factor since the questions were adapted to include up-to-date scientific information about the benefits of $\mathrm{F} / \mathrm{V}$ on the microbiome. It is necessary to begin to update baseline information for this area of nutrition science and to reflect this in future research studies. It is unclear if the parents have this newer knowledge despite the fact that similar information was sent home to parents in a handout prepared by this research team. This is also an avenue that could be studied as well since parents serve as the guardians and gatekeepers to food availability in the home environment. Even though, dietary behavior change, specifically at the school lunch in this study, was not significantly impacted, it is important to note that knowledge changes may be the first phase of progression needed to initiate behavior changes (Bentley et al., 2012).

Consistent findings in the literature suggest that enhancement of self-efficacy is important, but also a link to unlocking the next phase of behavior change adoption 
(Annesi \& Vaughn, 2017; Burke et al., 2005; Clark, Abrams, Niaura, Eaton, \& Rossi, 1991). Unfortunately, no changes were demonstrated in self-efficacy measures in either group and levels stayed relatively the same. This was somewhat of a surprise to the researchers since there was such a strong emergent theme of wanting recipes to practice eating more $\mathrm{F} / \mathrm{V}$ in this study, and the study was then adapted to add in recipes for the students. Perhaps, asking students to try the recipes at home and asking for more feedback would have stimulated a slight change in F/V intake, but sustainability of this would be undeterminable. In future studies, it will be key to emphasize self-efficacy in the design. This study aimed to impact self-efficacy with a repeated activity where students had the opportunity to pack a school lunch in an online video game. Data as to how frequently students accessed the game and participated were not collected; therefore this activity was perhaps underutilized.

The qualitative themes suggested taste was a strong motivator for selection of $\mathrm{F} / \mathrm{V}$. This might seem to be an obvious factor, but only moderate information in the literature supports this including a recent conclusion in a food selection study which stated, "Since the absolute number of healthy choices remained lower in children, this suggests that children may not yet be geared to modify their choices away from their natural tendency to choose unhealthy tasty foods" (van Meer, van der Laan, Viergever, Adan, \& Smeets, 2017, p. 325). While reasonable to assume, it is important to note that even though knowledge is present about eating foods with health benefits, if the food does not taste good, it is not likely well accepted in the diet. 
The method of presentation of information with repeated exposure on the computer compared to that of a one-time educational session may have contributed to the improvements in knowledge scores. The higher frequency of education sessions allowed for more information to be explained including how much the students need to eat, specific types of F/V to consume daily, and how they provide essential nutrients for positive health outcomes. The knowledge portion of the Farm to Table survey (Survey 1) was adapted to include questions that inquired about an understanding of new information related to the health benefits of $\mathrm{F} / \mathrm{V}$ as sources of prebiotic fiber, probiotics, and phytonutrients that all help in the development of a healthy microbiota and disease prevention. These concepts are unlikely available elsewhere in the health curriculum and most likely also new information for parents. These positive results show this adapted knowledge survey is a reasonable survey to include in the design of future studies, as it will provide information related to the understanding of current health benefits. These results also show that this new information is knowledge that middle school children probably are not presented with in school or at home.

The focus of Aim 2 was to determine if factors such as BMI, blood pressure, and gender would show an influence on the amounts and types of $\mathrm{F} / \mathrm{V}$ included in and consumed from home-prepared school lunches among middle school children ages 11-12 years. No significance was identified at baseline; however post study, significance was noted in the girls in both groups having more F/V servings present in their lunches compared to the boys. This was likely due to the fact that slightly more boys did not have $\mathrm{F} / \mathrm{V}$ servings noted in their lunches on the randomly selected day that observations were conducted. This was in contrast with our hypothesis gender would not affect the 
amounts of F/V included in and consumed from home-prepared school lunches among school children ages 11-12 years. However, only a small number of students did not have $\mathrm{F} / \mathrm{V}$ present in their lunch and this might not be a reasonable assumption in larger studies.

Published studies suggest there is a predictive relationship between lower BMI levels and higher in F/V intake; however, in this population, no significant difference was found even when accounting for age and gender by using calculated BMI z-scores. In previously, unpublished data by this research team, and consistent with findings from this study, no relationship was noted between BMI and gender and F/V servings consumed from school lunches. It is likely however, that the small sample size may be a limitation in further establishing a trend. Students in this sample size were of varying BMI ranges. The prevalence of overweight or obesity of the intervention group is consistent with national averages; 25 percent with a BMI z-score and a BMI for age at or above the $85^{\text {th }}$ percentile, compared to 30 percent of children overweight and/or obese in the US (CDC, 2008). In line with national averages, the control group had 30 percent of the students assessed as overweight or obesity, based on their BMI z-scores.

Students in this sample also had blood pressure levels consistent with national averages for children and adolescents in this age range. Blood pressure levels are evaluated based on a child's age and height percentile according to the NIH. A blood pressure percentile chart has been devised and more recently updated based on collected data in the NHANES studies in 1999-2000 (NIH, 2004). According to this data, for boys ages 11 and 12 years and girls in the same age range, blood pressure levels can be identified based on the child's height and systolic level separately from the diastolic 
level. In this study, a blood pressure of $\geq 120 / 80 \mathrm{mmHg}$ was considered too high, and parents would have been notified of this finding. No participants were noted to have a blood pressure at this level or above in this study. When looking at averages for both groups, the systolic level improved slightly at the post intervention measurement, but no clinical significance was noted. In addition, no significant difference between blood pressure and the presence of $\mathrm{F} / \mathrm{V}$ servings in the packed school lunch prepared was noted.

The qualitative responses from the focus group with the students identified themes related to the HBM constructs that support intake or avoidance of F/V servings in their individual school lunches. The constructs of cues to action and self-efficacy were most highlighted. These tenets provide the researcher with insights into behavior change that accompanies knowledge change. A resonating theme over the others that were gathered was the need for recipes that increase knowledge of how to improve the taste of F/V. It was also concluded from comments that despite the awareness that foods provide health benefits, if they didn't taste good, they were not likely to be a consistent component of their meals. After learning this information from these focus group sessions, the planned educational lessons were adapted to include recipes. Each student received at least one recipe for fruits and one for vegetables that were seasonal and that the student would be able to prepare with minimal adult supervision.

The results from parental involvement and correlation with student $\mathrm{F} / \mathrm{V}$ in the home packed lunch and consumption patterns were noted. Unfortunately, this research team expected more parental involvement, especially in the intervention group, and only about 67 percent of the participants returned the grocery-shopping list, which required at least one parent's participation. In the intervention group 38 percent did not return 
surveys three and four so there is incomplete information regarding what is available in the home of the intervention group participants. The apparent lack of parental involvement in the intervention group may have had an impact on the observed decline in F/V servings presence in home prepared lunches and consumption patterns, however more information is needed to further explain the full scope of this relationship.

Several data collection tools were used in this study to gather information about dietary intake of $\mathrm{F} / \mathrm{V}$ servings at different times (baseline and post study). This design was used in this pilot study to evaluate the usefulness of the survey forms for reported estimates of daily F/V servings. Each of the tools provided unique information and it was helpful to note the responses of the students and parents from an observational vantage point. The EATS and the YAQ, monthly recalls both involved parent report of dietary intake over the past month. This may have been too long of a time period for an accurate recall, and therefore, may not be the most useful in the plan of future studies. There was also some question of the value of the DILQ for children in this age population.

The constructs related to the combination of the three combined theories (SCLT, self-efficacy and HBM) contributed to the planned methods and educational design of this study. A combination of these foundations allowed for a broad scope of health promotion for changing $\mathrm{F} / \mathrm{V}$ intakes in middle school children, however, more could be done to see further significant changes. Environment or personal factors within SCLT, such as increased parental involvement, may be more substantially needed than expected. Peer impact and modeling within this group, may be necessary to see more positive outcomes as well. These construct levels of inclusion were possibly set too low in this study and further attention to this would be worthwhile in future studies. 


\section{Results Related to Health Theories}

The health theories serve to explain the results in this study. "Behavioral theories are important because they provide the framework and variables with which to understand why people do what they do" (Baranowski, 2006). The abstract findings were able to take more concrete shape when these established theories were incorporated to provide fundamental structure that guided the development of the educational tools in hopes of obtaining more successful behavior changes. A feedback loop was established as this study design involved a combination of three specific health theories in the methods, tools, assessments, and implementation phases in order to address F/V intake in middle school students. Questions in the qualitative focus groups were based on constructs of the HBM and themes that emerged from these findings shaped the development of the lessons and discussions during the intervention phase of this study. This served as a tailored approach to meet the needs of the audience and to take basic concepts described from their viewpoint and expand on those topics further during the interactive, multimedia education lessons. Therefore, the results from the focus groups were categorized according to the constructs of the HBM and then those same constructs carried over to the intervention phase as the foundation for each lesson. The quantitative measures in place were designed to look at self-reported and observed quantity of $\mathrm{F} / \mathrm{V}$ changes, changes in knowledge, and changes in self-efficacy constructs. The results show that knowledge changed, and that relates to personal factors incorporated in SCLT. Specific constructs of the HBM were measured such as self-efficacy, and while these levels did not change in either group, they provided information to help this research team reassess the value that must be placed on this construct in future studies. It is 
reasonable to assume that more emphasis in this area could lead to the actual behavior change goals that were established. Other HBM constructs were addressed in the qualitative portion of this study, but were not compared post study to see if perceptions changed. This may be one aspect to consider in future designs or tool development in order to capture specific information related to perceived seriousness, susceptibility, benefits, barriers, and cues to action.

While the inclusion of these three theories were helpful, there were some clearer implications of their impact upon further review of the results. For instance, the HBM constructs supported the ability of knowledge change, while self-efficacy remained stable, however other constructs were less adequately measured and this could have given more insightful information and application for this theory. In future studies, this could include awareness of changes in perceived benefits, barriers, and cues to action that would be key points to assess. The SCT utilized personal influences and impacts of peers and adults, which is known to be a powerful mediator on middle school students; however this was at a moderate intensity in this study. More information could have been elicited with just a few more steps and adaptable measurement tools. The selfefficacy influence was also likely too passive, and this research team could have incorporated a few more feedback points in order to delve deeper into confidence levels in $\mathrm{F} / \mathrm{V}$ intake changes.

\section{Limitations}

As previously mentioned, several limitations exist in this study. The sample size is small as this is a pilot study and more participants would be necessary to further substantiate the significant findings and positive trends. The convenience sample was 
mostly Caucasian students at a private school, and likely suggested more affluence; however, this was not measured in this study. While the students did have their own individual school provided computers, there would be instances when the Wi-Fi connection would be interrupted; thereby interfering with a planned health lesson. This may have affected results of those in the intervention group. The short duration of education sessions overall may have been a factor as noted by previous researchers. 


\section{CHAPTER VI}

\section{CONCLUSION}

Positive findings from this study suggest the education materials specially crafted for this age group were effective in changing knowledge about $\mathrm{F} / \mathrm{V}$ in the diets of middle school children. Students are growing more accustomed to learning on the computer as opposed to solely textbook delivery of information. An interactive approach with primarily web based health intervention lessons, was able to change the knowledge in the intervention group compared to that of the control group that had only minimal exposure to the topic information. Changes in the delivery mode of education may be a more effective way to educate students in the next generations as media changes and technology continues to advance. It is a promising marker to note that education can be learned in this manner for this subject area especially as more health related information may be reduced in school curricula, possibly due to budget cuts, or simply not enough class time to include the information. Teachers, in some states, are pressured to teach, according to what is included in standardized tests, and this may impact the amount of time that is allocated for health education, therefore, presentation of information in a format that is engaging and that can be assigned is promising. The prevalent issue of overweight and obesity beginning in youth worldwide, dictates that more diet information is needed to help young people make food choices consistent with health based standards set forth by the IOM and USDA.

Results from this pilot study show that while some food intake related behaviors were unchanged, some trends toward significance are especially noteworthy. The observed increase in student reported relative amounts of $\mathrm{F} / \mathrm{V}$ is significant in the "Farm 
to Table" Relative Amount of Consumption survey in the intervention group from baseline to post study. The control group demonstrated a positive trend from baseline to post study as well in this same survey. These findings suggest more total $\mathrm{F} / \mathrm{V}$ intake may have occurred post study within the entire day, possibly due to the generalized focus on the topic presented in the school curricula, even though changes were not observe within the F/V servings present in the packed school lunch post study. Further evidence supports the relationship between the presence of the F/V servings in the lunch and the consumption amount, and should serve as promising information for parents and students. This relationship suggests that having the F/V servings in the school lunch will more often result in consumption of the servings, and packing these foods in the lunch should be practiced daily for the most health benefits.

Reports from the parents are especially helpful in fully understanding what the child is offered in comparison to their intake of $\mathrm{F} / \mathrm{V}$ servings. Often parents may feel that the child doesn't like a certain F/V so they stop offering it, however, data suggests that repeated exposures to foods are necessary for acceptance to occur (Birch, Savage, \& Ventura, 2007). This strategy may cause a temporary increase in food waste and cost; however, the potential benefit of preventing a debilitating condition is likely to outweigh the financial expenses and loss of quality of life.

More education is needed for parents to fully understand what children's needs are for daily F/V intake. As parents are the main gatekeepers in providing the food in the home, education must occur for them as well. Attempts to educate and involve parents in this study included: (1) A request for parents to complete two surveys bout what F/V servings are available for their children and the estimated consumption of these foods, (2) 
Information sheets that highlighted key points that were presented to their child in the learning modules, and (3) A request for parent partnership, with their child, to write a grocery list and return it to the school for gym class credit. There was no crosscheck to assess if parents received or read the information sheets, and many students did not submit the grocery list assignment; there was no consequence for the missing assignment. Therefore, involvement of parents was questionable in the intervention group and appeared to be less than that of parents in the control group because fewer, only 62.5 percent returned surveys 3 and 4 , whereas 76 percent of the control group parents returned these surveys. It is likely that an intervention effect beyond the observed changed in knowledge occurred if there was better parent participation in the intervention group.

Even though there was a significant impact on knowledge about the health benefits of $\mathrm{F} / \mathrm{V}$ intakes, there was not an observed behavior change related to $\mathrm{F} / \mathrm{V}$ serving amounts in or consumption from home prepared school lunches. Factors that may have contributed to this result include the amount of materials and activities in the curriculum; more time to develop content with additional activities that reinforce learned knowledge and application into action might result in significant behavior change of bringing more $\mathrm{F} / \mathrm{V}$ servings and eating them in school lunch. The design did not capture data related to who packed the students' lunches. It was assumed the parents packed the lunches. If the student participants were allowed to pack their lunches, as a study activity, the presence of $\mathrm{F} / \mathrm{V}$ post study may have been correlated to the increased knowledge scores. This study also may have shown different results if it was initiated at the beginning of the 
semester instead towards the end of the school year; student focus may have been diverted towards plans for the summer versus implementing dietary changes.

Future Studies

Future study designs are justified to include a longer implementation phase that incorporates additional activities to allow students to "practice what they learn" and reinforce the concepts presented. This may allow for increased self-efficacy scores and perhaps further progression toward positive health behavior changes. More feedback from the facilitators that hold students accountable for assignments will communicate the importance of this educational learning tool and ultimately strengthen the impact. This study design is suitable for public school initiation and can derive further information about $\mathrm{F} / \mathrm{V}$ consumption patterns in school prepared and home prepared meals as well.

With regard to the present school in this study, another study of interest would compare intakes on the days when hot lunches are brought to the school from an outsourced vendor with the days when the students bring home-prepared school lunches. This would provide opportunity for students to make choices based on what they have learned from a selection of foods that may be unfamiliar to them. This will allow researchers to better understand how students make choices and may result in different recommendations that will help students increase $\mathrm{F} / \mathrm{V}$ choices.

Additional qualitative measures would be helpful in the form of interviews or focus groups at the end of the intervention to allow for more dialogue about what the students learned and how they changed their behaviors. This would require the researchers to be forthcoming about what foods were being studied, however, and may increase the chance of false-positive results of F/V intake. Any additional information 
gleaned from the perspective of the students in middle school would be valuable when designing future programs.

A study that involved only the parents would also be a valuable research avenue to pursue. Providing the same nutrition intervention information to the parents instead of the students may be even more impactful on the diet of the students since parents are the gatekeepers of the home environment. This would require more time by the parents, however because the information can be delivered online allows for more flexibility in transferring the information. A second phase to this would be to having parents then empower their child to pack lunches on their own and follow the guidelines described to measure changes in intake of $\mathrm{F} / \mathrm{V}$.

Additionally, the role of environment may impact the exposure a child has to $\mathrm{F} / \mathrm{V}$ in places such as the home and in the school. It is feasible to review the environmental culture, which is tied to eating F/V and influencing food selections. This also is related to family support, peers, rewards in the school and reinforcement of eating behaviors and would be beneficial to consider in future studies.

Despite the limitations that have been identified, the findings from this pilot study do provide a solid foundation for future study designs. For example, the comprehensive inclusion of theoretical frameworks and methods that were developed and used to evaluate student lunches for content worked very well and can easily be reproduced by other researchers with similar study design. While the number of subject data points varied, the average number of students was 16 in the intervention group and 13 in the control group. Due to absences from school, rushed transitions from class to class, or preference to not respond by the student, some data points were missing. The number (n) 
of responses or data points is listed on each of the tables and figures that are present in the analysis results. The inclusion of health theories for educational materials is useful and necessary. The combination of the three theories used in this study provided structure for curriculum development and framework for topic discussions. The blended theory approach seemed to be a positive tool and demonstrated trends toward promising outcomes in some areas. More application of these frameworks combined may be necessary to show further inclusion of these constructs in child based, nutrition research. In conjunction, the number of constructs available for measure could be increased. For example, measuring the perceived benefits and barriers in an adaptive scale could be useful information to capture. Constructs of SCT could include more active engagement with parents and peers and adequate assessment of these interactions would be quite helpful.

\section{Applications for Practice}

The F/V servings estimated from this pilot study with all of the evaluation tools described in this dissertation, are equal to reported national averages, however are lower than the current recommendations of at least five $\mathrm{F} / \mathrm{V}$ servings in a day. These findings are applicable to the practice of nutrition within the fields of education, community, and clinical practice. The following considerations based on the results of this study include:

- Updated $\mathrm{F} / \mathrm{V}$ information is necessary to help increase the presence and consumption of $\mathrm{F} / \mathrm{V}$ in the diets' of middle school children.

- Parental involvement at high levels may be necessary for their child to achieve the daily-recommended intake of $\mathrm{F} / \mathrm{V}$ servings. 
- Online education about $\mathrm{F} / \mathrm{V}$ intake is possible among children in similar learning environments with access to Canvas ELS.

- Reference to health theories are helpful frameworks when developing educational materials aimed for middle school students.

- The presence of F/V servings is related to the amount of consumption as demonstrated in this study. Therefore, if these foods are present in the meal, it is more likely they will be consumed. 


\section{CHAPTER VII}

\section{APPENDICES}

\section{APPENDIX A: School Lunch Federal Register Report}

\section{Table 5: National School Lunch Program: Current Requirements Compared to}

\section{Final Rule Requirements for a 5-Day School Week}

\begin{tabular}{|c|c|c|c|c|c|c|c|c|c|}
\hline \multirow[b]{2}{*}{ Grade Levels } & \multicolumn{3}{|c|}{$\begin{array}{l}\text { Current Requirements: Traditional } \\
\text { Food-Based Approach }\end{array}$} & \multicolumn{3}{|c|}{$\begin{array}{l}\text { Current Requirements: Enhanced } \\
\text { Food-Based Approach }\end{array}$} & \multicolumn{3}{|c|}{ Final rule $^{e}$} \\
\hline & $\mathrm{K}-3^{\mathrm{b}}$ & $4-12^{b}$ & $7-12^{\mathrm{c}, \mathrm{d}}$ & $\mathrm{K}-3^{\mathrm{b}, \mathrm{d}}$ & $4-12^{b}$ & $7-12$ & $\mathrm{~K}-5$ & $6-8$ & $9-12$ \\
\hline Fruit (cups) & $2.5^{f}$ & $3.75^{f}$ & $3.75^{f}$ & $3.75^{f}$ & $4.25^{\mathrm{b}}$ & $5^{f}$ & 2.5 & 2.5 & 5 \\
\hline Vegetable (cups) & & & & & & & 3.75 & 3.75 & 5 \\
\hline Dark Green & NS & NS & NS & NS & NS & NS & 0.5 & 0.5 & 0.5 \\
\hline Orange & NS & NS & NS & NS & NS & NS & 0.75 & 0.75 & 1.25 \\
\hline Legumes & NS & NS & NS & NS & NS & NS & 0.5 & 0.5 & 0.5 \\
\hline Starchy & NS & NS & NS & NS & NS & NS & 0.5 & 0.5 & 0.5 \\
\hline Other & NS & NS & NS & NS & NS & NS & 0.5 & 0.5 & 0.75 \\
\hline Additional Veg to Reach Total & NS & NS & NS & NS & NS & NS & 1 & 1 & 1.5 \\
\hline \multirow[t]{2}{*}{ Grain/Bread (oz eq) } & $8(\min$ & $8(\min$ & $8(\min$ & $10(\min$ & $12(\min$ & $15(\min$ & & & \\
\hline & $1 /$ day $)^{g}$ & $1 /$ day $)^{g}$ & $1 /$ day $)^{g}$ & $1 /$ day $)^{g}$ & $1 /$ day $)^{g}$ & $1 /$ day $)^{g}$ & $8-9$ & $8-10$ & $10-12$ \\
\hline Meat/Meat Alternates (oz eq) & 7.5 & 10 & 15 & 7.5 & 10 & 10 & 8-10 & $9-10$ & $10-12$ \\
\hline Milk (cups) & 5 & 5 & 5 & 5 & 5 & 5 & 5 & 5 & 5 \\
\hline
\end{tabular}

${ }^{a}$ Requirements and recommendations are for meals as offered for a 5-day school week.

${ }^{\mathrm{b}}$ Minimum portion sizes

${ }^{\mathrm{c}}$ Recommended potion sizes for the Traditional Food-Based Menu Planning approach.

d Optional grade configuration.

${ }^{\mathrm{e}}$ See Table 3 and Table 3 footnotes for additional detail. Final rule standards shown in this table are after full phase-in (SY 2014-2015).

${ }^{\mathrm{f}}$ Two or more servings of fruit, vegetables, or both a day.

${ }^{\mathrm{g}}$ Must be enriched or whole grain.

${ }^{\mathrm{h}}$ Two or more servings of fruit, vegetables, or both a day, plus an extra half-cup over the 5-day school week.

(USDA, 2012b). 


\section{APPENDIX B: Daily Reference Intakes (DRI) and Tolerable Upper Intakes}

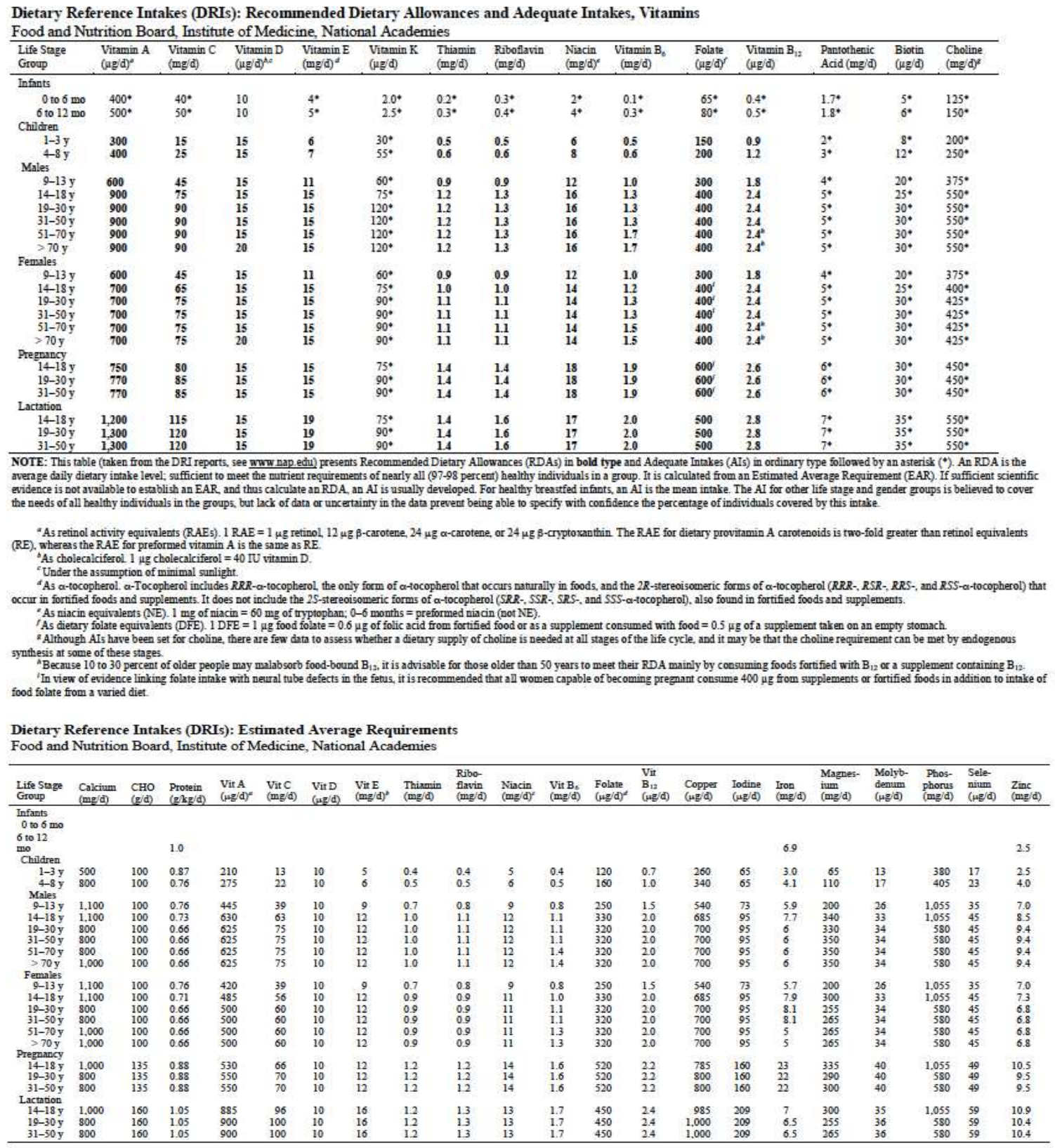

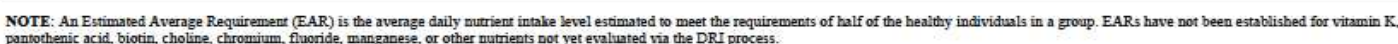

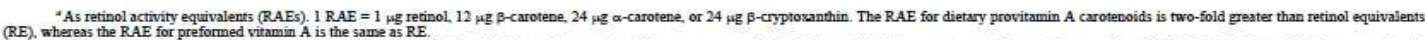

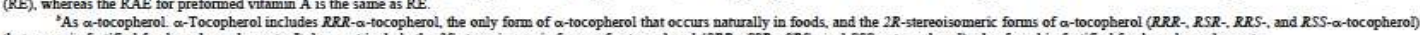
occur in fortified foods and supplements. It does not include the 2S-stereoisomeric forms of $\alpha$-tocopherol (SRR-. SSR-, SRS., and SSS- $\alpha$ - - -tocopherol), also found in fortified foods and supplements.

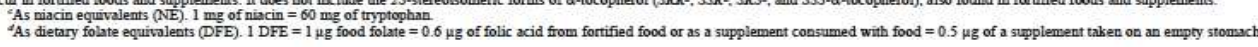

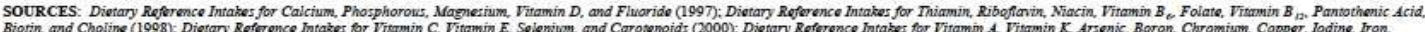

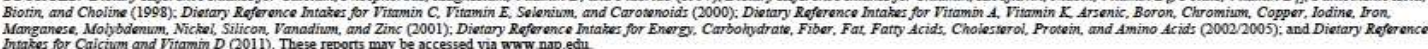

(Cengage, 2004; IOM, 2017). 
Tolerable Upper Intake Levels (UL) for Vitamins

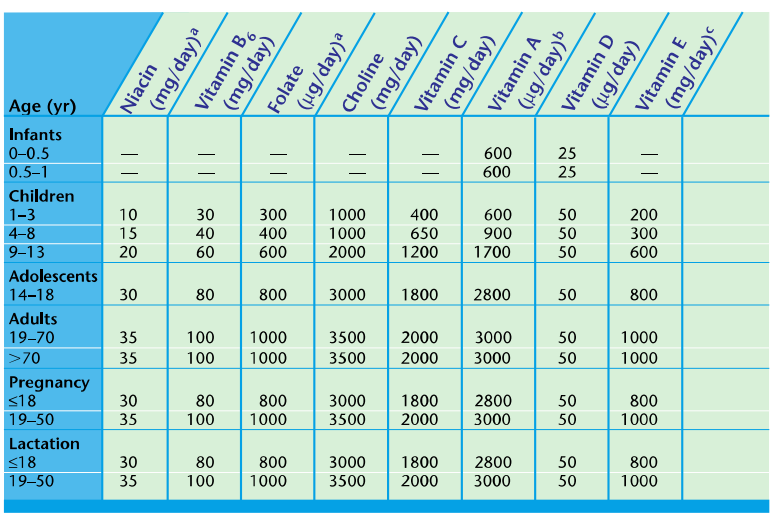

aThe UL for niacin and folate apply to synthetic forms $\quad{ }^{b}$ The UL for vitamin A applies to the preformed vitamin only. obtained from supplements, fortified foods, or a combination cThe UL for vitamin E applies to any form of supplemental.
of the two.

Tolerable Upper Intake Levels (UL) for Minerals

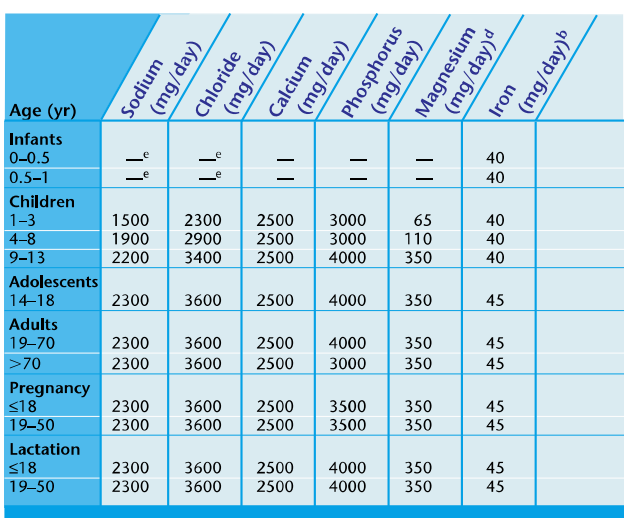

${ }^{\mathrm{d} T \text { The UL }}$ for magnesium applies to synthetic forms obtained from supplements or drugs only.

- Sounce or mage shoul be from human milk (or formula) and food only.

listed with a dash (-) because eftablished for vitamins and minerals not listed and for those age group

level of intake. All nutrients can have adverse effects when intakes are excessive.

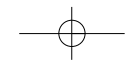


APPENDIX C: Approved protocol submitted to the Institutional Review Board (IRB) prior to the initiation of the study and data collection.

\section{IMPACT ON FOOD INTAKE WITH A MULTIMEDIA EDUCATIONAL TOOL INCORPORATING THE HEALTH BELIEF MODEL AMONG MIDDLE SCHOOL CHILDREN \\ Judith Ernst, DMSc, RD \\ School of Health \& Rehabilitation Sciences (SHRS) \\ Indianapolis, IN}

Sub-Investigators and their affiliations here:

$\begin{array}{ccc}\text { JoAnne Whelan, MS, RD } & \text { Lisa K. Staten, PhD } & \text { Brent Arnold, PhD } \\ \text { SRHS } & \text { Fairbanks School of Public Health } & \text { SRHS } \\ \text { IUPUI } & \text { IUPUI } & \text { IUPUI } \\ & & \\ & & \\ \text { Paula Magee, PhD } & \text { Jessica Kanis, MD } & \text { Laurie Trevino, MS } \\ \text { Education } & \text { IU School of Medicine } & \text { IU School of Medicine } \\ \text { IUPUI } & \text { IU } & \text { GCRC }\end{array}$

Table of Contents:

Study Schema

1.0 Background

2.0 Rationale and Specific Aims

3.0 Inclusion/Exclusion Criteria

4.0 Enrollment/Randomization

5.0 Study Procedures

6.0 Reporting of Adverse Events or Unanticipated Problems involving Risk to Participants or Others

7.0 Study Withdrawal/Discontinuation

8.0 Statistical Considerations

9.0 Privacy/Confidentiality Issues

10.0 Follow-up and Record Retention

11.0 References 


\subsection{Background}

Over $30 \%$ of children are overweight and obese in the United States (CDC, 2008). Comorbid conditions related to obesity in this population are also on the rise as many youth are at risk for elevated blood pressure and cardiovascular disease (Kelly et al., 2015). In 2009, approximately $30 \%$ more children were diagnosed with Type 2 diabetes over the previous decade with links to disproportionate dietary and activity changes, along with lesser dietary quality as factors (Cree-Green et al., 2013; Hamman et al., 2014). In conjunction, many children fall short in receiving adequate amounts of fruit and vegetable $(\mathrm{F} / \mathrm{V})$ servings daily as $90 \%$ of children do not consume adequate amounts of vegetable servings, and $60 \%$ of children do not take in enough fruit servings as recommended by the government dietary guidelines (CDC, 2014). More efforts to combat poor health and diet with promotion of increased $\mathrm{F} / \mathrm{V}$ in the diet may shift the energy sources away from high fat and high sugar food items. In addition, the inclusion of more $\mathrm{F} / \mathrm{V}$ in the diets of children will increase soluble fiber intake and subsequently create a healthier microbiota, which may also be necessary for long-term positive health outcomes (Principi \& Esposito, 2016).

Educating youth about healthy eating choices in the school environment, specifically middle school age students as they are still forming eating habits, is the beginning step toward helping children understand the relationship between diet and health (IOM, 2001; USDA, 2016a). Identifying an educational approach that may improve this method of processing information is important for learning in a way that improves attentiveness and retention of information. A psychological framework that includes social cognitive learning theory and the health belief model (HBM) outlines a dynamic approach to the transfer of information surrounding dietary intake of $\mathrm{F} / \mathrm{V}$ for this population (Kay \& Kibble, 2016; Kehm et al., 2016).

\subsection{Rationale and Specific Aims}

Nutrition education for middle school age children that is focused on the importance of $\mathrm{F} / \mathrm{V}$ utilizing the constructs of the HBM for subsequent behavior change to increase $\mathrm{F} / \mathrm{V}$ intakes in daily diets has not been reported; therefore, a gap in scientific literature exists. In this pilot study we will describe the home prepared school lunch intake of F/V among middle school student's ages 11-12 years, and how the dietary inclusion of $\mathrm{F} / \mathrm{V}$ is impacted with repeated multimedia educational instruction and interactions provided over six weeks, in an intervention group compared to a group without repeated intervention.

Specific Aim 1: Does the impact of an educational intervention on the types and amounts of $\mathrm{F} / \mathrm{V}$ included in and consumed from home-prepared school lunches differ among middle- school age children at ages 11-12 years at a parochial Indianapolis-area school 
who receive either educational modality A (repeated educational video plus interactive feedback) or educational modality B (one time educational video without interactive feedback)?

Specific Aim 2: Do factors such as BMI, blood pressure, and gender relate to the amounts and types of $\mathrm{F} / \mathrm{V}$ included in and consumed from home-prepared school lunches among middle school children ages 11-12 years?

Specific Aim 3: Do the qualitative responses from a focus group with students, ages 1112 years, identify themes related to the HBM constructs that support intakes or avoidance of $\mathrm{F} / \mathrm{V}$ servings in individual school lunches?

\subsection{Inclusion/Exclusion Criteria}

Criteria for Inclusion:

- Boy or girl between the ages of 11-12 years.

- All students that are part of the regular classroom and participate in the classroom activities

- Student at Immaculate Heart of Mary School, Indianapolis, IN.

- Agree to participate in the study as evidenced by the parental consent and student assent forms.

Criteria for Exclusion:

- Student or parent that does not sign consent forms.

- No student will be excluded for physical or mental learning differences. If a student is part of the normal classroom, he/she is able to participate in this study.

\subsection{Enrollment/Randomization}

One middle school class will be selected as the intervention group and another class will serve as the control group from the convenience sample of students at Immaculate Heart of Mary School. One class will receive the repeated intervention educational modality, and the other class will serve as the control group and receive a one-time education session for comparison. A maximum of 55 students and their parents will be enrolled in this study.

The location of Immaculate Heart of Mary School is:

317 E. $57^{\text {th }}$ Street

Indianapolis, IN

46220

(317) 255-5468 


\subsection{Study Procedures}

Students and their parents are asked to participate in this research study. Student data will include information from surveys, personal data, observational data, and focus group discussion. Parental data will include survey responses regarding foods they offer in the home (Survey 3 ) and assist their child with completing at home surveys with regards to their child's food intake (Survey 4).

\section{Data Collection (see Table 1 for flow chart to describe the specific aims, data collection, outcome measure, and statistical analysis)}

\section{Recruitment and Enrollment}

- Initial email sent to parents and students introducing the study

- Informed consent from parents; hard copy sent and received via "kid mail"

- Informed assent from students; hard copy sent and received via "kid mail"

\section{Surveys}

- Baseline knowledge of students will be collected adapted from the "Farm to Table" tool validated in children for F/V intake (see Appendix A-Survey Number 1) (Jamelske, Bica, McCarty, \& Meinen, 2008).

- Day in the life questionnaire (DILQ) completion by students during their study hall time at school; students will be asked to draw their lunch meal (see Appendix B-Survey Number 2) (Edmunds \& Ziebland, 2002; Moore et al., 2007)

- Parental behaviors for food provisions and foods offered to children will be measured with adapted "Eating at America's Table for Fruits and Vegetables" (see Appendix C-Survey 3) (Murphy, White, Viani, \& Mosby, 2016; Subar et al., 2001; Thompson et al., 2004; Tooze et al., 2010; LaBrosse \& Albrecht, 2013).

- Child assessment of food intake will be collected with and adaption from the Youth Adolescent Food Frequency Questionnaire (see Appendix D-Survey Number 4) (Borradaile et al., 2008)

\section{Personal Data}

- Student height will be collected in a private area during the student's study hall period. Students, in stocking feet, will stand erect on a health care grade scale (Detecto (C) and height in inches will be noted as a level wood block is brought to the top of the head and then recorded; two individual measures will be taken (Lohman, Roche, \& al., 1988).

- Student weight will be collected in a private area during the student's study hall period. Students, fully clothed in stocking feet, will step onto a standard, health care grade, calibrated scale (Detecto (C) and weight in pounds will be noted and then recorded; two individual measures will be taken (R. S. Gibson, 2005).

- Body Mass Index (BMI) calculations will be assessed from height and weight measures using the United States standardized data method for growth assessment (CDC, 2016, 2017; Wells \& Fewtrell, 2006). 
- Student blood pressures will be collected with digital blood pressure machines in a private area during the student's study hall or physical education class periods (Kelly et al., 2015; NIH, 2007; Setayeshgar et al., 2017). If a student's blood pressure is found to be elevated; greater than or equal to $120 / 80 \mathrm{~mm} \mathrm{Hg}$, the child's parent will be notified by telephone to make them aware of this finding the same day. Contact with the child's primary care physician will be suggested.

\section{Meal Observational Data}

- At baseline, student lunches brought from home will be assessed for F/V food items on three different unannounced days, Tuesday through Thursday. On one occasion, the lunch will be digitally photographed with an iPad device; food items placed next to a ruler for an accurate scale of measurement will be linked to participants with assigned research numbers. Observations will be made by a trained researcher or registered dietitian and recorded for:

$\circ$ 1) Presence of F/V (yes or no)

- 2) Type of $F / V$

$\circ$ 3) Approximate number of servings of $F / V$ in half cup serving sizes

$\circ$ 4) Percentage of consumption of $F / V$ at conclusion of lunch period

- At the end of the study period, approximately six-eight weeks after the initial data collection, on an unannounced day Tuesday through Thursday, student lunches, brought from home will be assessed for F/V food items. On this occasion, the lunch will be digitally photographed with an iPad device; food items placed next to a ruler for an accurate scale of measurement will be linked to participants with assigned research numbers. Students and their lunches will be observed by a trained researcher or registered dietitian and recorded for:

○ 1) Presence of F/V (yes or no)

- 2) Type of $F / V$

- 3) Approximate serving size of $F / V$ in half cup serving sizes

$\circ$ 4) Percentage of consumption of $F / V$ at the conclusion of the lunch period

\section{Focus Groups}

- Focus groups will be conducted as part of the study hall period. This activity is optional for students and they will be asked to check a box on the consent/assent forms to verify willingness to participate or not. Students may participate in the rest of the other activities within the study without participating in the focus group discussion. It is anticipated that the number of students in the Focus Group will not exceed 28 students. Students will have minimal risks associated with data collection. The entire sessions will be audio recorded. The focus group will be conducted pre intervention and will be led by the study's co-investigator and follow a scripted set of questions that probe the constructs of the Health Belief Model and F/V intake (See Appendix E-Focus Group). 
- The audio recording will be stored on a flash drive, which will be locked in a file cabinet on the IUPUI campus for three years. After this time period the data will be destroyed. No identifying factors will be linked to the students and their statements.

The school will not have access to any of the study information provided and gathered in any format. Participation will not affect their standing in the school.

\section{Interventions:}

Educational interventions (See Table 2) will be conducted during the scheduled physical education class or study hall. Students will be asked to bring their school assigned Chromebook computers to these classes. They will participate in approximately 15-20 minutes of PI and/or Co-PI led educational sessions which will include interactive videos, games, or trivia related to content discussed in class. A flow chart of all data collection points and interventions is listed in Table 3.

- Lesson 1: General fruit and vegetable overview (provided to both the intervention and control groups)

- Lesson 2: Perceived susceptibility and perceived seriousness of poor health conditions related to poor dietary intake of $\mathrm{F} / \mathrm{V}$ (intervention group only)

- Lesson 3: Perceived Benefits and Perceived Barriers to eating healthy foods (intervention group only)

- Lesson 4: Self-Efficacy and Cues to Action (intervention group only)

- Lesson 5: Student generation of Public Service Announcements (PSA's) in groups of 5 students (intervention group only)

- Lesson 6: Wrap up and review previous session information and PSA's (intervention group only)

- Lesson 7: Review PSA's (intervention group only)

- Lesson 8: Review PSA's (intervention group only)

Table 4 outlines child and parent requirements for the study procedures. Note focus group participation is optional.

\subsection{Reporting of Adverse Events or Unanticipated Problems involving Risk to Participants or Others}

The process for reporting adverse events (AE) or unanticipated problems involving risk to participants or others will be handled by the lead investigator. Submission of AEs/unanticipated problems will be through the IUPUI IRB office via email (IRB@iu.edu) and phone (317-274-7930). If an AE occurs it will be immediately reported to this agency

\subsection{Non-Participation/Study Withdrawal/Discontinuation}


Students who choose not to participate will view the educational materials with participating students as a class activity; however, baseline and outcome data will not be collected from non-participating students. During the time when data collection occurs (surveys, personal data, observation data, focus groups, etc.) non-participating students will be given the option to complete homework or other related independent work.

Students are participants and may withdraw at any time during the study. A student or parent/caregiver can contact the site coordinator (JoAnne Whelan) and request removal from the study.

The process for the participants to withdraw from the study will be given directly to them and their parents/caregiver. Data after withdrawal from the study will not be collected.

\subsection{Statistical Considerations}

This is a pilot study and results will be used to determine the sample sizes for future like studies. Data will be analyzed with SPSS version 2013. The statistical methods that will be used include descriptive statistics, paired t-tests, analysis of Covariance, Pearson coefficient of correlation, and qualitative analysis (see Table 1). The statistical power, or the alpha level of the study will have a probability set at 0.05 and a confidence interval of $95 \%$. We anticipate statistical significance to be determined with at least 12 subjects in each group. Any possible deviations from significance may be due to a small population or brevity of the study protocol.

\subsection{Privacy/Confidentiality Issues}

Participant privacy is assured with the assignment of a study number that will be used to identify all data collection points instead of identifiable labels (name or date of birth). All data received and collected will be held in privacy and confidentiality upheld at all times. No labels used to identify individuals will be used in presentations, conferences, or publications. Hard copies of data forms will be stored in a locked file cabinet in a locked office. Electronic data will be stored on a secure drive at IUPUI for three years then destroyed.

\subsection{Follow-up and Record Retention}

The study is scheduled to last approximately 9-10 weeks from the start of recruitment through the end of the last data collection (March 2017-May 2017). The documents and audio recordings will be stored in a locked office at IUPUI. The duration of data record retention will be three years and all materials will be destroyed thereafter. 


\section{Description of the specific aims, data collection, and outcome measures}

Specific Aim

1. Does the impact of an educational intervention on the types and amounts of $\mathrm{F} / \mathrm{V}$ included in and consumed from home-prepared school lunches differ among middleschool age children at ages $11-12$ years at a parochial Indianapolis-area school who receive either educational modality A (repeated educational video plus interactive feedback) or educational modality $\mathrm{B}$ (one time educational video without interactive feedback)?

2. Do factors such as BMI, blood pressure, and gender relate to the amounts and types of $\mathrm{F} / \mathrm{V}$ included in and consumed from home-prepared school lunches among middle school children ages 11-12 years?

3. Do the qualitative responses from a
Data Collection

1. Survey 1

2. Survey 2

3. Survey 3

4. Survey 4

5. Observation of school lunch meals pre (data will be collected on 3 different days to determine baseline variability) and post intervention (one day).

1. Demographic data

2. Height

3. Weight

4. BMI

5. Gender

6. Observations of school lunch meals pre and post intervention.

1. Focus group pre and post recordings of
Outcome Measure Statistical

Analysis

1. Knowledge and 1. Repeated

Self-Efficacy pre Measures Anova and post

intervention.

2. Dietary recall of

2. Repeated

number of

Measures Anova

servings of $\mathrm{F} / \mathrm{V}$

3. Number of $F / V$

offerings provided

3. Repeated

to students pre and

Measures Anova post intervention.

4. Number of $F / V$

servings consumed 4. Repeated

in child's diet pre Measures Anova and post

intervention.

5. Number of 5. Repeated offerings of $\mathrm{F} / \mathrm{V} \quad$ Measures Anova

pre and post intervention.

1. Descriptive data

1. Descriptive statistics

2. Relationship between BMI, 2. Analysis of blood pressure, Covariance; gender and Pearson amount/types of Coefficient of $\mathrm{F} / \mathrm{V}$ intake. correlation

1. Themes related

1. Qualitative to the inclusion or Statistics 
focus group with themes.

student's ages 11-12

years identify themes

related to the HBM

constructs that

support intake or avoidance of $\mathrm{F} / \mathrm{V}$

servings in their

individual school

lunches? exclusion of $\mathrm{F} / \mathrm{V}$

in the diet. 
Weekly lessons for interventions including the date, the specific details, justification, the researcher responsible for implementation, and completion date.

\section{Specific Details}

First lesson

Second lesson

Third lesson

Fourth lesson

Fifth lesson

Sixth lesson

Seventh lesson

Eighth lesson
Peer Impact and repetition

$\mathrm{F} / \mathrm{V}$ general info for $\mathrm{F} \& \mathrm{~V}$ 123

-Serving size

-How many cups

-Measure with

Your fist

Perceived

Susceptibility/Perceived

Seriousness

Perceived

Benefits/Perceived Barriers

Self-Efficacy/Cues To

Action

Build PSA's in class

Peer Impact and repetition

Peer Impact and repetition

\section{Justification}

Myplate.gov

CDC

$\mathrm{NIH}$

(LaBrosse \&

Albrecht, 2013)

Myplate.gov

CDC

$\mathrm{NIH}$

(LaBrosse \&

Albrecht, 2013)

Myplate.gov

CDC

$\mathrm{NIH}$

(LaBrosse \&

Albrecht, 2013)

Myplate.gov

CDC

$\mathrm{NIH}$

(Gupta et al., 2013; Co-Investigator Hong et al., 2008;

Rogers et al., 2016)

IN Education

Standards

(Gupta et al., 2013;

Hong et al., 2008;

Rogers et al., 2016)

IN Education

Standards

(Gupta et al., 2013;

Hong et al., 2008;

Rogers et al., 2016)

IN Education

Standards

(Gupta et al., 2013;

Hong et al., 2008;

Rogers et al., 2016)
Implementation

Co-Investigator

Co-Investigator

Co-Investigator

Co-Investigator

Co-Investigator

Co-Investigator

Co-Investigator

(May 15) 
Study events listed in order by study event and week data collection point. The highlighted squares indicate both groups will complete the activity.

\begin{tabular}{|c|c|c|c|c|c|c|c|c|c|}
\hline Study Event & $\begin{array}{l}\text { Weeks } \\
1\end{array}$ & 2 & 3 & 4 & 5 & 6 & 7 & 8 & 9 \\
\hline Consent/Assent & $\mathrm{X}$ & & & & & & & & \\
\hline \multicolumn{10}{|l|}{ Data Collection } \\
\hline Survey 1 & & & $\mathrm{X}$ & & & & & $\mathrm{X}^{* *}$ & \\
\hline Survey 2 & & & $\mathrm{X}$ & & & & & $\mathrm{X}^{* *}$ & \\
\hline Survey 3 & & & $\mathrm{X}$ & & & & & $\mathrm{X}^{* *}$ & \\
\hline Survey 4 & & & $\mathrm{X}$ & & & & & $\mathrm{X}^{* *}$ & \\
\hline $\begin{array}{r}\text { Height, Weight, } \\
\text { BMI, Blood } \\
\text { pressure }\end{array}$ & & & $\mathrm{X}$ & & & & & & $\mathrm{X}$ \\
\hline Focus Group & & & $\mathrm{X}$ & & & & & & \\
\hline $\begin{array}{r}\text { Meal } \\
\text { Observation }\end{array}$ & & & $\mathrm{X}, \mathrm{X}, \mathrm{X}$ & & & & & & $\mathrm{X}$ \\
\hline $\begin{array}{r}\text { Education } \\
\text { Intervention } 1\end{array}$ & & & & $\mathrm{X}$ & & & & & \\
\hline $\begin{array}{r}\text { Education } \\
\text { Intervention } 2\end{array}$ & & & & & $\mathrm{X}$ & & & & \\
\hline $\begin{array}{r}\text { Education } \\
\text { Intervention } 3\end{array}$ & & & & & $\mathrm{X}$ & & & & \\
\hline $\begin{array}{r}\text { Education } \\
\text { Intervention } 4\end{array}$ & & & & & & $\mathrm{X}$ & & & \\
\hline $\begin{array}{r}\text { Education } \\
\text { Intervention } 5\end{array}$ & & & & & & $\mathrm{X}$ & & & \\
\hline $\begin{array}{r}\text { Education } \\
\text { Intervention } 6\end{array}$ & & & & & & & $\mathrm{X}$ & & \\
\hline $\begin{array}{r}\text { Education } \\
\text { Intervention } 7\end{array}$ & & & & & & & $\mathrm{X}$ & & \\
\hline $\begin{array}{r}\text { Education } \\
\text { Intervention 8* }\end{array}$ & & & & & & & & $\mathrm{X}^{*}$ & \\
\hline
\end{tabular}

*Conducted on Monday; outcome data collection after this point.

**Outcome data collection begins on Tuesday of this week. 
Study procedures and parent/child requirements.

\begin{tabular}{|c|c|c|}
\hline Procedure & $\begin{array}{c}\text { Parent } \\
\text { Requirement }\end{array}$ & $\begin{array}{c}\text { Child } \\
\text { Requirement }\end{array}$ \\
\hline $\begin{array}{l}\text { Height, Weight, Blood } \\
\text { Pressure }\end{array}$ & & $X$ \\
\hline Survey 1,2 & & $\mathrm{X}$ \\
\hline Focus Group* & & $X$ \\
\hline Survey 3 & $X$ & \\
\hline Survey 4 & $X$ & $\mathrm{X}$ \\
\hline Meal Observations & & $X$ \\
\hline Education Modules 1-8 & & $\mathrm{X}$ \\
\hline
\end{tabular}




\section{APPENDIX D: Approved informed parental consent and child's assent forms}

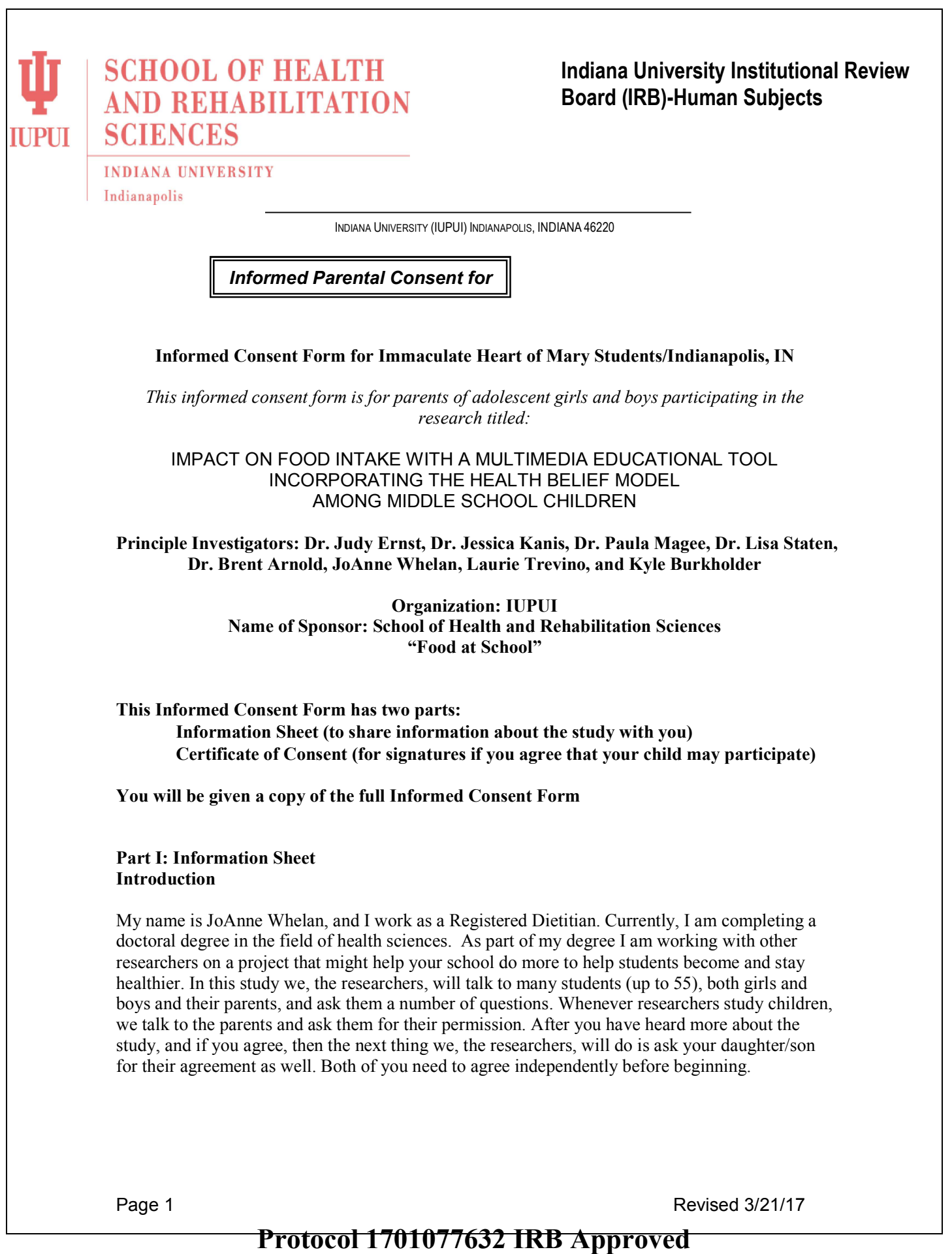




\section{Purpose}

It is possible that children are not meeting national standards for food or nutrient intake. This may cause poor health in the future. In this study we, the researchers will talk to girls and boys about what they know about being healthy, including their diet and what foods they eat. The purpose of this project is to use the information the children provide to create educational tools.

\section{Selection of Participants}

Our research team wants to talk to many students (11-12 years of age) about health and wellness as possible (with a maximum of 55 students and their parents). You and your child are invited to participate, at this time, because you live in an area where electronic educational learning through CANVAS may occur in the future.

\section{Voluntary Participation}

Participation in this study is strictly voluntary. You and your child are not required to participate. You do not have to agree that your child can talk to us. You can choose to say no and any services that you and your family normally receive at this school will not be changed. The researchers know that the decision can be difficult when it involves your child. Also, it can be especially hard when the research includes personal topics such as the foods that your child eats. You can ask as many questions as you like and we will take the time to answer them.

\section{Procedure}

All students in the same homeroom class will be provided education. There is one education module that students will view. This module will be viewed during their physical education and/or study hall course between now and the end of the quarter. Students will be given access and instruction for CANVAS, a web-based electronic learning system that requires the use of school provided Chromebook and the Internet. This module will take 10-15 minutes to complete.

For those who have agreed to participate in this study, the following study procedures will occur (See study procedure Table 1):

Prior to the education module described above, your child's height, weight, body mass index, and blood pressure will be measured in a private area during study hall. Students will be asked to complete two surveys given on paper or online. All students will be invited to participate in a focus group. Then, surveys 3 and 4 will be completed by the parent/student at home. Finally, meal observations will occur during the regularly scheduled lunch on unannounced days at the beginning and end of the study for dietary assessment. These procedures will be repeated after the class is finished with the education module.

Table 1: Study procedures

\begin{tabular}{|c|c|c|}
\hline Procedure & Parent Requirement & Child Requirement \\
\hline $\begin{array}{c}\text { Height, Weight, } \\
\text { Blood Pressure }\end{array}$ & & $\mathrm{X}$ \\
\hline Survey 1, 2 & & $\mathrm{X}$ \\
\hline Focus Group & $\mathrm{X}$ & $\mathrm{X}$ \\
\hline Survey 3 & $\mathrm{X}$ & $\mathrm{X}$ \\
\hline Survey 4 & & $\mathrm{X}$ \\
\hline Meal Observations & & $\mathrm{X}$ \\
\hline Education Module 1 & & \\
\hline
\end{tabular}




\section{Surveys}

$>$ Participants may skip any questions or ask for clarification at any time.

$>$ Survey 1 will gather information about your child's food knowledge about eating and food intake; in addition to their gender, birthdate, and ethnicity/race.

$>$ Survey 2 will allow your child to describe the foods he/she eats through pictures.

$>$ Survey 3 will require you, the parent, to provide information about the foods that are available at home for your child to eat.

$>$ Survey 4 consists of questions that will give us more information about the foods that your child eats. You, the parent, will be asked to help your child complete this form.

\section{Focus Groups}

A focus group is a discussion session with a moderator and participants that are asked questions about a topic. The moderator will be one of the researchers. There may be as many as 28 students in the group. Students do not need to participate in the focus group to participate in the rest of the study. For students who have agreed to participate in the focus group, this will take place during one study hall period. During the focus group, students will be asked to provide feedback about foods they eat and why. This session will be audio recorded for transcription. All information will be confidential and no one other than the researchers will have access to the recordings. No information will be reported back to the school. The recordings will be on file for 3 years in a locked cabinet on a disk drive at IUPUI then they will be destroyed.

\section{Meal Observations}

At the beginning of the school lunch period, students will be asked to place the contents of their home prepared school lunch meal on a plate provided by the researchers. Then the students will gather to the side for a short activity lasting approximately 5-7 minutes. The researchers will record contents and/or photograph the plate for review. Students will be asked to leave any remaining, uneaten food items on the plate at the end of the meal for review.

\section{Duration}

The time commitment of this study is during regularly scheduled school hours. This study will occur beginning now through the end of the quarter.

\section{Risks and Discomforts}

The primary or main risk is a loss of confidentiality. There is a chance that you or your child may share personal or confidential information about your health and nutrition beliefs related to foods. However, we do not desire this to happen and you or your child do not have to answer questions that may make you feel uncomfortable in any way. We may find that your child may have high blood pressure (greater than or equal to $120 / 80 \mathrm{~mm} \mathrm{Hg}$ ). If that happens, you, the parent, will be notified by telephone. If this occurs, it will be recommended that you follow up with your primary care physician.

\section{Benefits}

There are educational benefits to you and your child, for participating in this research study. These findings may help guide further development of educational tools for the greater community and improve the health of you, your child, and others.

\section{Reimbursements}

There is no compensation provided to you or your child for your participation this research study, however, your child's entire class may earn extra gym time during a scheduled study hall period as decided by the physical education instructor.

\section{Confidentiality}




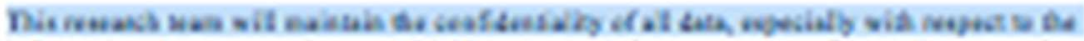

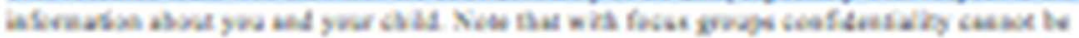

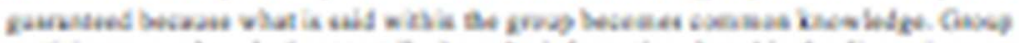

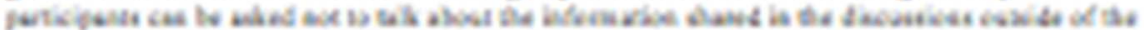

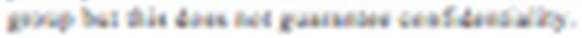

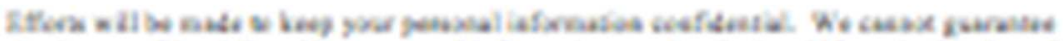

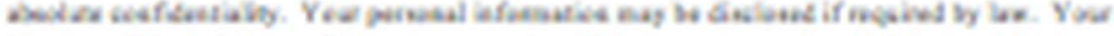

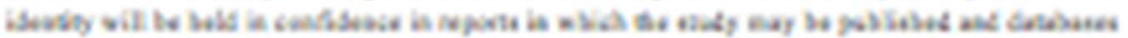

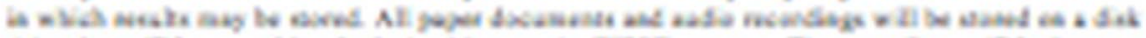

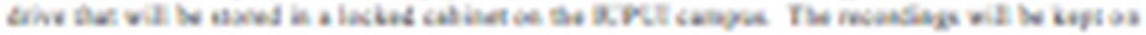

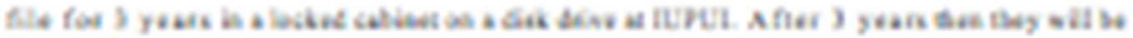
sewooned

\section{Shariat of Rewarob Nakioge}

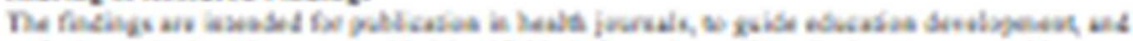

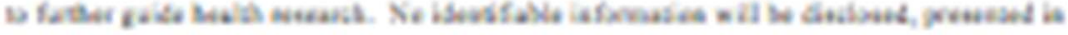

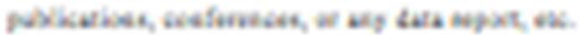

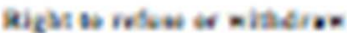

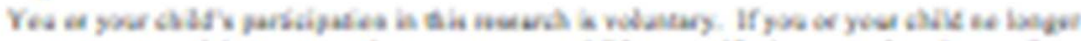

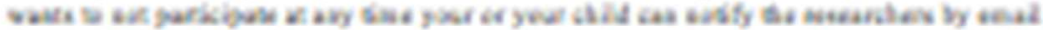

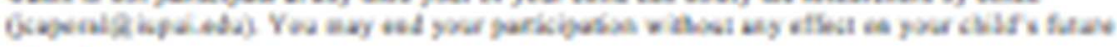
edscates.

\section{Wha beatect}

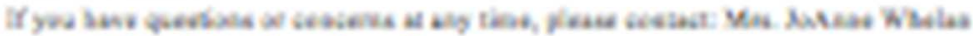

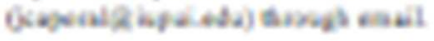

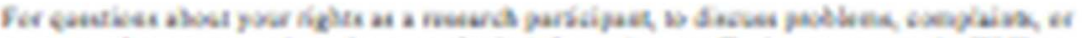

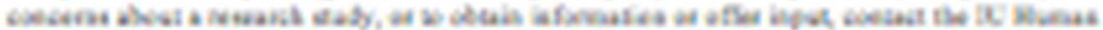
Sabsut Orfos at 


\section{PART II: Certificate of Conseat}

\section{Certificate of Consent}

I have been asked to participate aloag with my child and I give cousent for my child to participute in this resesrch study, which will involve completing four survers at the begining and four surveys at the end of the stedy, along with focus groups, meal observations, sad persoanal data collection. I have read the foregoing information, or it hes been read to me. I have bad the opportaniry to ask questions about it and any questions that I have asked bave been answered to my satisfaction. I voluntarily conseat for myself and for my child to participate as in thir study.

Initial this line if it is ol for your chald to participste in the focms group.

Print Name of Pareat or Geardian

Siguature of Pareat of Guardian

Date

Desymoath/year 


\section{Indiana University Assent to Participate in Research}

\section{IMPACT ON FOOD INTAKE WITH A MULTIMEDIA EDUCATIONAL TOOL INCORPORATING THE HEALTH BELIEF MODEL AMONG MIDDLE SCHOOL CHILDREN}

Mrs. Whelan and other researchers are doing a research study. A research study is a special way to learn about something. The researchers are doing this study because they are trying to find out more about foods that middle school students eat at home and at school. The researchers would like to ask you, the student, to be in this research study with a maximum of fifty-five students and their parents.

Why am I (the student) being asked to be in this research study?

You (the student) are being asked to be in this research study because the researchers would like to learn more about the types of foods that you eat at home and at school.

What will happen during this research study?

There are some things that might happen if you (the student) are in the study. This study will take place at Immaculate Heart of Mary of School. It will last for approximately 8-9 weeks.

If you (the student) want to be in this study, here are the things that will be asked of you during part of your physical education class or study hall:

$>$ Complete surveys (4 surveys two separate times; and will take 15 minutes or less each time)

$>$ Review online materials (1 lesson that will take 15 minutes or less in gym class and/or study hall).

$>$ Take off your shoes and step onto a scale to find your weight and your height (Two times, and will take 5-10 minutes each time)

$>$ Place a blood pressure cuff around your arm and take your blood pressure (Two times, and will take 5 minutes each time). If your blood pressure is greater than or equal to $120 / 80 \mathrm{~mm} \mathrm{Hg}$ we will call your parents to tell them. We will suggest your parents contact your doctor.

$>$ Have your lunch observed at school during your lunchtime (1-4 times for 5-10 minutes each).

$>$ Optional activity: Participate in a question and answer conversation (focus group) that will be audio recorded, during your study hall or gym class for 15-20 minutes. The recording will be kept for a maximum of three years then deleted/destroyed. There may be as many as 28 students in the group. You do not have to participate in the focus group to be a part of the rest of the study.

\section{Are there any bad things that might happen during the research study?}

Sometimes bad things happen to people who are in research studies. These bad things are called "risks." One risk of being in this study is that someone may find out information about you that you do not want anyone to know, like how tall you are, how much you weigh, or what you like to eat. The researchers will be careful to weigh you in a place 
separate from anyone else and will keep your weight information private. The researchers will ask everyone in your discussion group not to share what is discussed with anyone. Therefore, these things will probably not happen to you. All of your information will be kept private and nothing learned, as part of this study will be reported back to the school. Things may happen though that the researchers don't know about yet. If anything bad does happen, we will make sure that you get any help that you need.

\section{Are there any good things that might happen during the research study?}

Sometimes good things happen to people who are in research studies. These good things are called "benefits." The benefits of being in this study might be that you will learn more about the foods that you eat that will help you be healthy as you continue to grow. The researchers don't know for sure if you will have any benefits. We hope to learn something from you that will help other people some day.

\section{Will I get money or payment for being in this research study?}

You will not get any money for being in this research study. Your entire class may earn extra gym time for participation on this project during your scheduled study hall time, as decided by the physical education instructor.

\section{Who can I ask if I have any questions?}

If you have any questions about this study, you can ask your parents or guardians or Mrs. Whelan, the researcher. Also, if you have any questions that you didn't think of now, you can ask later or ask the researcher the next time you see him or her.

\section{What if I don't want to be in the study?}

If you don't want to be in this study, you don't have to. It's up to you. If you say you want to be in it and then change your mind, that's OK. All you need to do is tell the researchers that you don't want to be in it anymore. You can also email Mrs. Whelan and let her know that you would not like to participate. No one will be mad at you or upset with you if you don't want to be in it.

\section{My choice:}

If I write my name on the line below, it means that I agree to be in this research study.

\section{Check this box if you will participate in the focus group.}


Participant's signature

Participant's printed name

Signature of person obtaining assent

Name of person obtaining assent

Date

Date 
APPENDIX E: Parent handouts given to the students after the education lessons

\section{F \& V - 1,2,3 \\ (Fruits \& Vegetables- 1, 2, 3)}

- Fruits and vegetables are low in the diets of children and adults.

- Fruits and vegetables provide nutrients including vitamins, minerals, and fiber.

- There are $\mathbf{1 3}$ vitamins essential for normal growth and function and play a role in digestion, immune function, and more.

- Minerals are necessary for normal development including nerve and muscle function, hormone production, and more.

- Fiber is a substance necessary for normal gut functions including absorbing nutrients, fueling the gut cells, and maintaining healthy bacteria levels.

- Girls' ages 9-13 years, need about 2 cups per day total and boys need closer to $2 \frac{1}{2}$ cups. One $1 / 2$ cup is equal to the size of your fist.

- Aim for 1 fist at breakfast, 2 fists at lunch, and 3 fists at dinner to get close to the recommended amount needed daily.

- Leafy green salad counts for 1 cup for every 2 cups of leafy greens toward the daily amount needed. This is due to the high water content of these foods.

- Juice that is $100 \%$ fruit juice counts as well, but limited to 4-6 ounces daily, because it is lacking fiber.

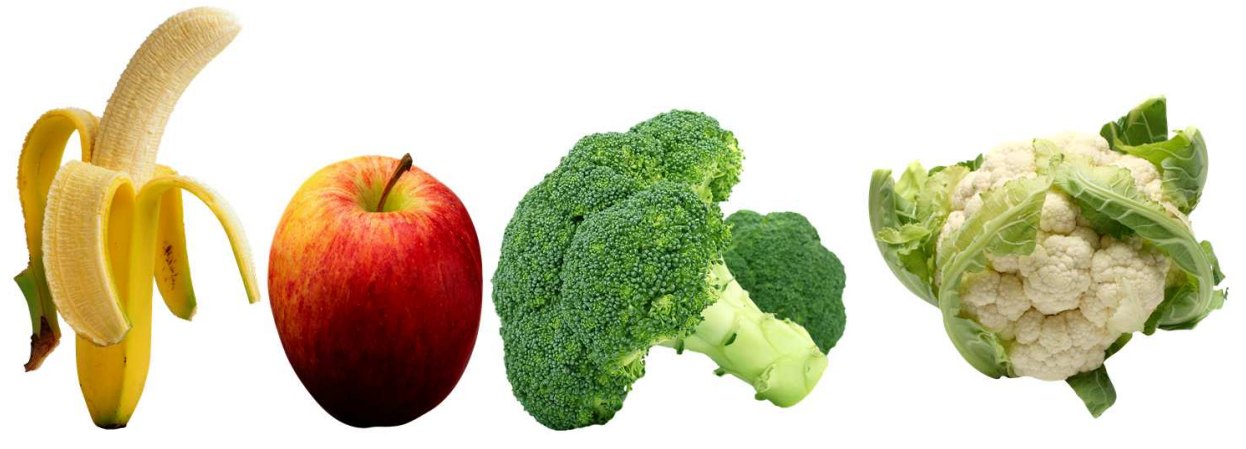


$1 / 2$ cup blueberries at breakfast

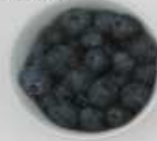

1 clementine at snack time

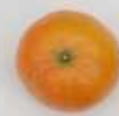

$1 / 2$ cup sugar snap peas at snack time

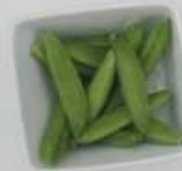

1 container applesauce at lunch

\section{$1 / 2$ sweet pepper} at lunch

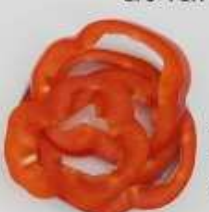

$1 / 2$ baked sweet potato +6 asparagus spears at dinner

\section{9-13 Tear Olds}

Sample Lunch with at Least $2-1 / 2$ cups of Fruits \& Vegetables

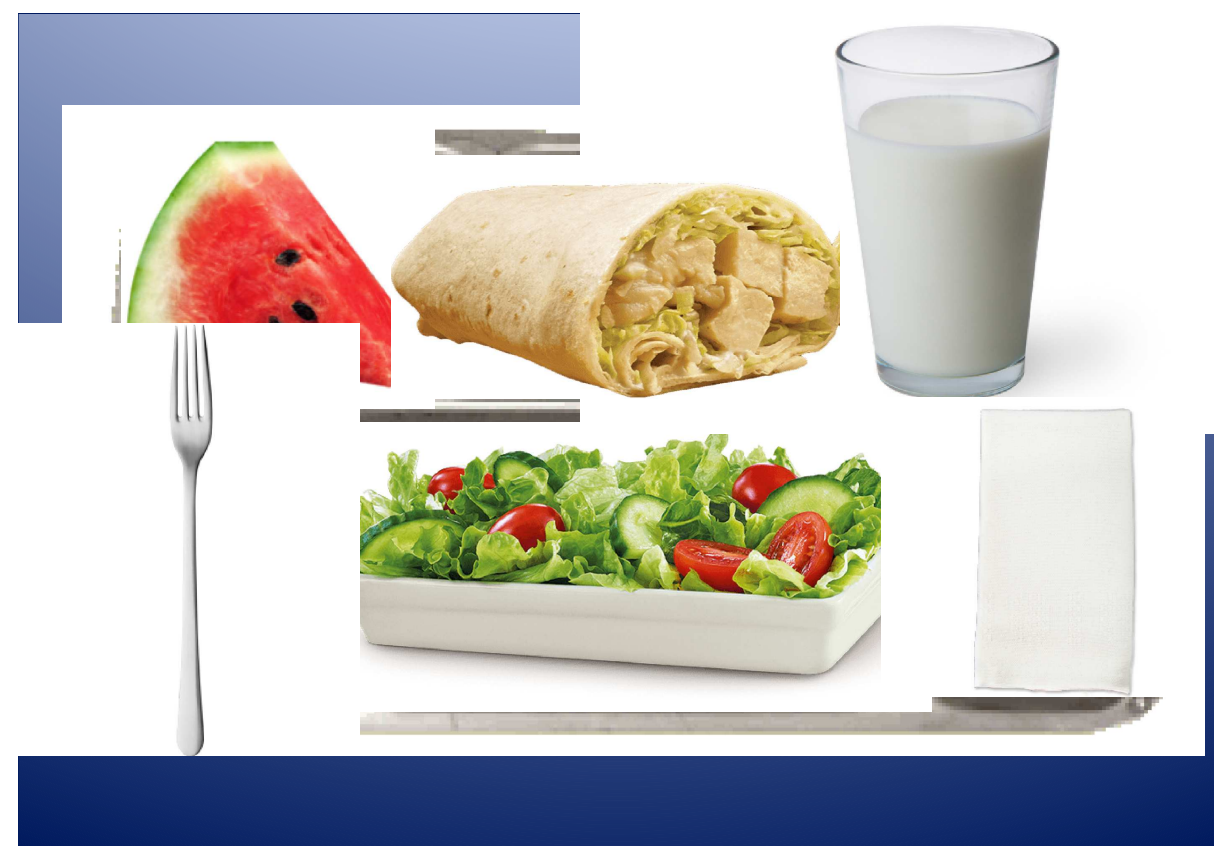




\section{$F \& V-1,2,3$ \\ (Fruits \& Vegetables- 1, 2, 3)}

- Sometimes there are things or beliefs that might get in the way of eating fruits and vegetables on a regular basis. This is called a barrier. There are many barriers that people could believe that gets in the way from adding these foods to their diet.

- Some barriers include: Perceived high cost of fruits and vegetables; no available places that are convenient to buy fruits and vegetables; people eat lesser amounts of foods at home and more often from restaurants or fast food places that do not include fruits and vegetables.

- There are benefits, or good outcomes, that come from eating fruits and vegetables. These include: healthy body, reduced risk of infections, improved heart function, and lower risk of some cancers.

- Fiber helps reduce blood cholesterol levels and may lower risks of heart disease.

- Vitamin C is important for growth and repair of all body tissues, helps heal cuts and wounds, and keeps teeth and gums healthy.

- Folate (folic acid) helps the body form red blood cells.
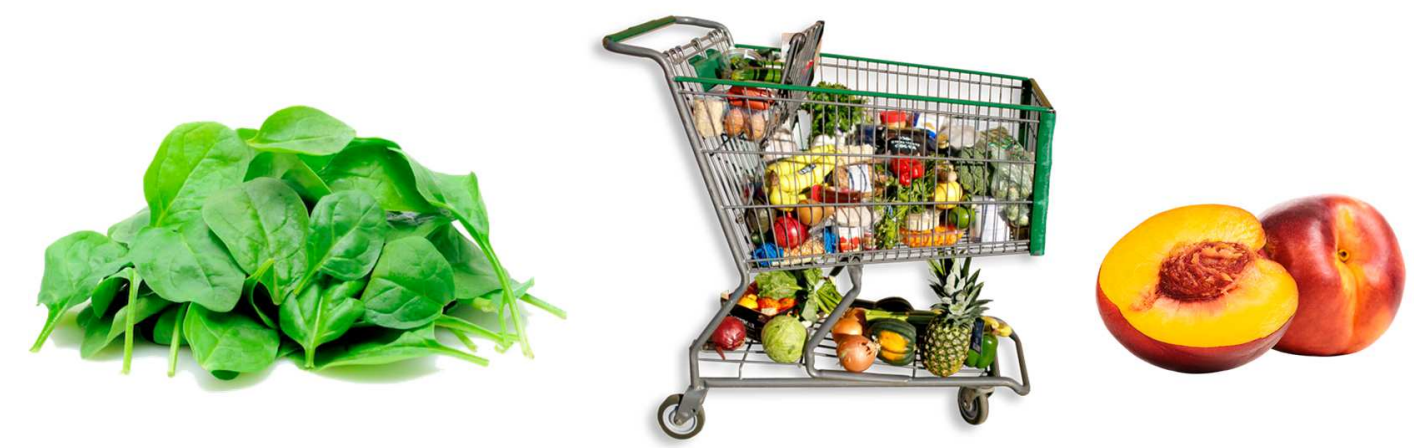

Time to grocery shop with your child! Please have your child fill in items for each category by finding recipes that you will want to make for the week, and then go shopping together. Please complete this activity within the next week. 

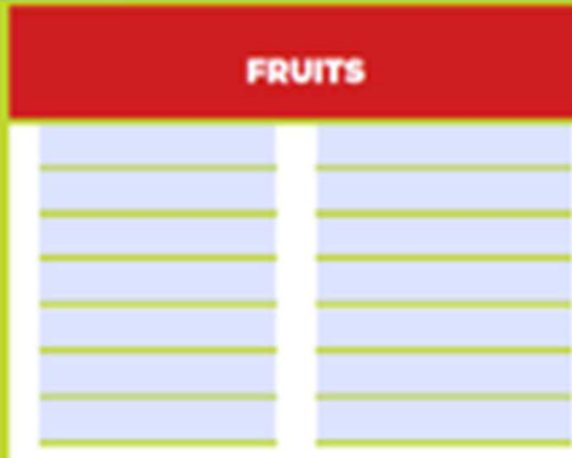

DAIRY

auk rocuri, cuteso

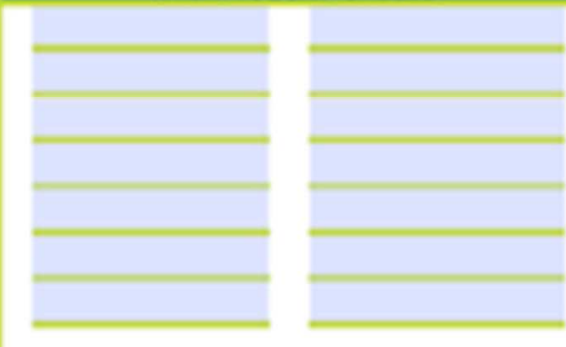

PROTEIMFOODS

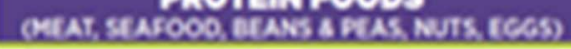
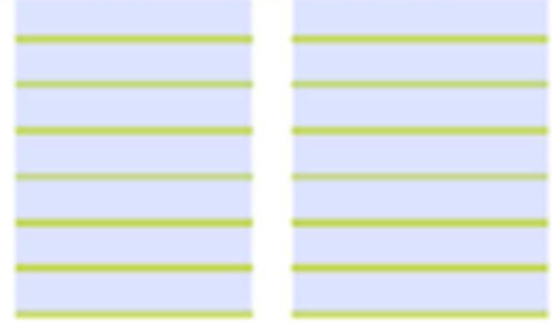

GRAINS

aegaos. pastas. Aict. creenis)

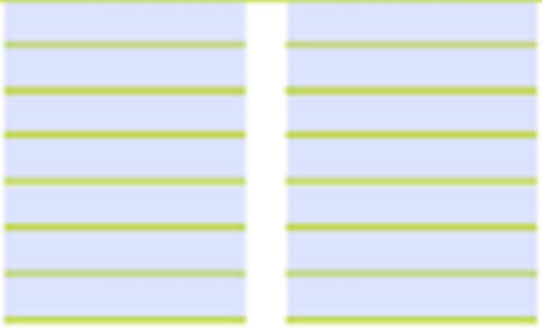

\section{VECETABLES}

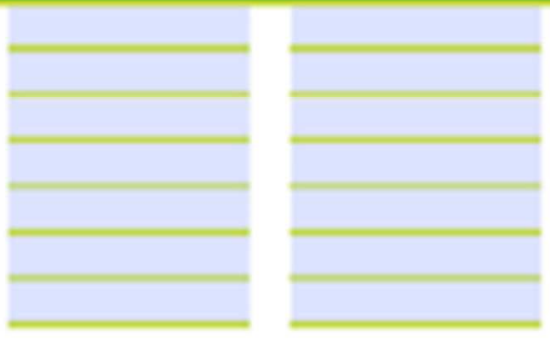

\section{Orita}
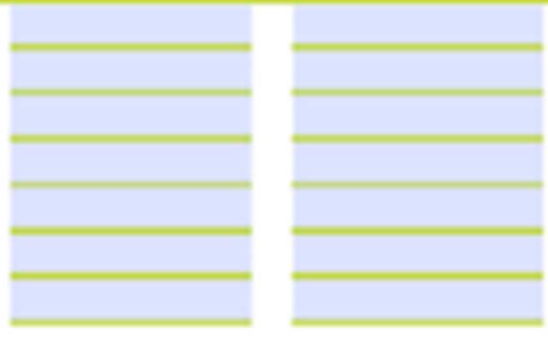

NOTES: 


\section{$F \& V-1,2,3$ \\ (Fruits \& Vegetables- 1, 2, 3)}

- Self-efficacy is a term to describe your individual belief that you can do certain behaviors or carry out an action. It is similar to shooting a basketball or hitting a baseball. Your thought about the behavior might lead to a feeling about it, then a decision to act, which would lead to results and ultimately impact your belief.

- Ways to increase your self-efficacy with eating fruits and vegetables is to gain knowledge, give support, try it out, and practice the behavior until it becomes a habit.

- Start with eating fruits and vegetables at home at 2 meals

- Try new fruits and vegetables at school and also at a friend's house. PRACTICE THIS! Then it becomes habit.

- Cues-to-action is the "push" it takes to make you "act" on the behavior.

- In groups, students are to create a public service announcement that supports adding fruits and vegetables to the diet.
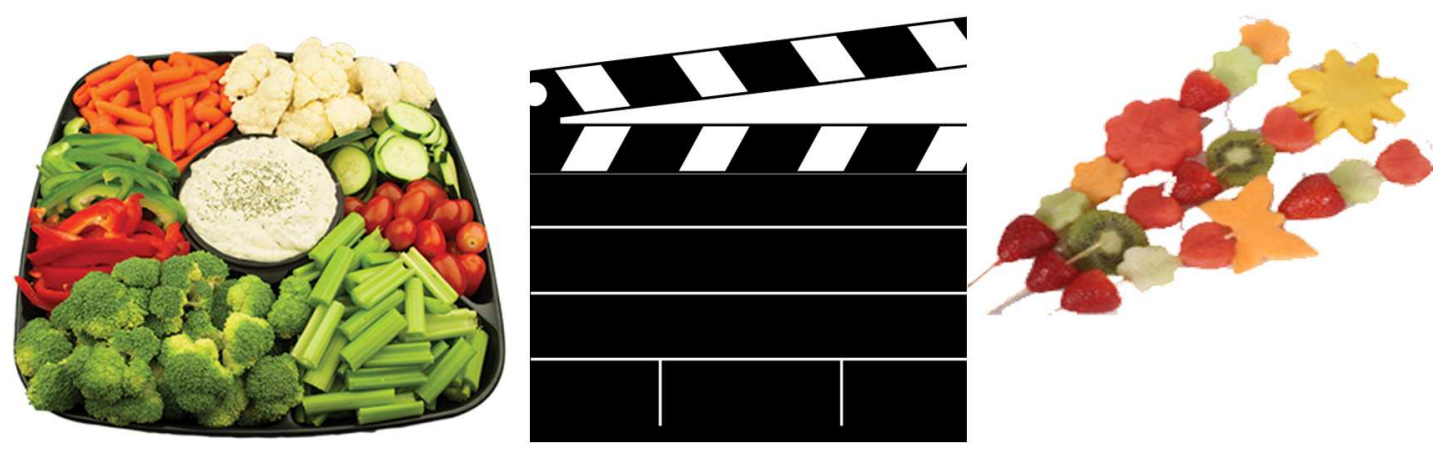

Guidelines for Public Service Announcement 
In your group you will plan what you want other student's your age to learn about nutrition, specifically about fruits and vegetables. You will have time in class to prepare for this on Friday, May 12th. You can make notes in a Google document and share this with your group. You will present these messages on May 15th and May 19th in gym class. They can be about 20 seconds long. You can be as creative as you would like. Everyone should have a short speaking part that states what you have learned from the modules. 


\section{APPENDIX F: Focus group discussion probe questions for each group}

Focus group questions adapted from "Bone and Soy" focus group based on the HBM (Robinson, Bielmowicz, Rodgers, Wong \& Konzelmann, 2008).

\begin{tabular}{|l|l|}
\hline Health Belief Model Construct & Selected Discussion Probes \\
\hline $\begin{array}{l}\text { Perceived susceptibility of poor health related } \\
\text { to lack of fruits and vegetables in your diet }\end{array}$ & $\begin{array}{l}\text { Why do you think some people eat healthy and } \\
\text { why do some people not eat healthy foods? If } \\
\text { you eat fruits and vegetables are you eating } \\
\text { healthy or not eating healthy? Will you have } \\
\text { health problems if you don't eat healthy? }\end{array}$ \\
\hline $\begin{array}{l}\text { Perceived severity of poor health related to low } \\
\text { intake of fruits and vegetables }\end{array}$ & $\begin{array}{l}\text { Do you eat fruits and vegetables? If yes, do you } \\
\text { eat them because they are good for you? }\end{array}$ \\
\hline Perceived benefits of fruits and vegetables & $\begin{array}{l}\text { What are the health benefits of fruit and } \\
\text { vegetables foods? Do you eat fruits and } \\
\text { vegetables for the health benefits? }\end{array}$ \\
\hline $\begin{array}{l}\text { Perceived barriers of consuming fruits and } \\
\text { vegetables }\end{array}$ & $\begin{array}{l}\text { Do you go food shopping with our parents? Do } \\
\text { you buy fruits and vegetables when you go } \\
\text { shopping? Why or why not? }\end{array}$ \\
\hline Cues to Action for health prevention & $\begin{array}{l}\text { Some people do not eat any fruits and } \\
\text { vegetables or they eat low amounts. Do you } \\
\text { think that they will have poor health? If yes, } \\
\text { what can they do to help prevent any health } \\
\text { problems. What kind of health problems might } \\
\text { they have? }\end{array}$ \\
\hline $\begin{array}{l}\text { So you every cook recipes that have fruits and } \\
\text { vegetables as ingredients? Do you think if you } \\
\text { vegetables } \\
\text { ingredients would you eat more fruits and } \\
\text { vegetables? }\end{array}$ \\
\hline
\end{tabular}




\section{APPENDIX G: Surveys/Forms}

\section{APPENDIX G-1: The adapted "Farm to Table" survey (Survey 1) questions}

\section{Survey 1}

\section{School Student Survey}

This is not a test and it will not affect your grades. Please answer every question, telling us what you really think. If you have questions you may ask your teacher or the adult in charge during this survey.
1. I am a
2. What ethnic group do you belong to? (Circle one)
3. How much do you like fruit? $\square$ (Circle one)

Male
or
Female
(Please
circle one)

African-American

A lot

Asian-American

Caucasian $\square$

Hispanic

Native American

American Indian

\section{Other}

5. How
much do
you like
tasting new
fruits?
Please tell
how you
feel about
tasting new
fruit.
$\square($ Circle
one)

A little

Not very much

Not at all

4. When you try a new fruit for the first time, how much do you usually like it? $\square$ (Circle one)

A lot

A little

Not very much

Not at all
6. Will you taste a fruit if you don't know what it is? $\square$ (Circle one)

7. Will you taste a fruit if it looks strange? $\square$ (Circle one)

A lot
A little
A little

Not very much

Not at all

Not very much

Not at all
8. Will you taste a fruit if you have never tasted it before?

A lot

A little

Not very much

Not at all

A lot

A little

Not very 
much

Not at all

$\begin{array}{ll}\text { 9. When you } & 10 . \text { When you are at } \\ \text { are at a } & \text { school, will you try a } \\ \text { friend's } & \text { new fruit? } \\ \text { house, will } & \end{array}$

, will you try a new fruit?

A lot

\section{Not very} much

\section{Not at all}

13. How much do you like vegetables?

A lot

A lot

\section{A little}

A little

Not very much

Not at all

Not at all usually like it?

Not very much

Not at all
11. When you are at home, will you try a new fruit?

A lot

A little

Not very much

Not at all

14. When you try a new vegetable for the first time, how much do you
15 . When you try a new vegetable for the first time, how much do you usually like it?

A lot

\section{A little}

Not very much

\section{Not at all}

12. How many times have you tried a new fruit since school started this year?

Never

1 time

2 times

3-4 times
16. How much do you like tasting new vegetables?

A lot

A little

Not very much

Not at all 
17. Will you taste a vegetable if you don't know what it is?

A lot

A little

Not at all
19. Will you taste a vegetable if you have never tasted it before?

A lot

A little

Not very much

Not at all
20. When you are at a friend's house, will you try a new vegetable?

A lot

A little

Not very much

Not at all

Not very much

\section{Not at all}

21. When you are at school, will you try a new vegetable?

A lot

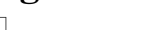

Not very much

Not at all

\section{A little}

22. When you are at home, will you try a new vegetable?

A lot

A little

Not very much

Not at all
26. Fruits and vegetables that are high in Vitamin A are Red and white

Blue and light brown

Dair Yellow-
23. How many times have you tried a new vegetable since school started this year?

Never

1 time

2 times

3 times

At least 4 times

27. Why do I need to eat food?

I need food for energy and to grow.

I need food ONLY because it
24. Imagine a meal with a hotdog in a bun and a glass of milk. What food group is missing?

Please circle one.

Dairy

Fruits and Vegetables

Meat

Grains

28. What is a benefit of eating fruits and

vegetables?

They
may help
protect
against
certain types
of cancer




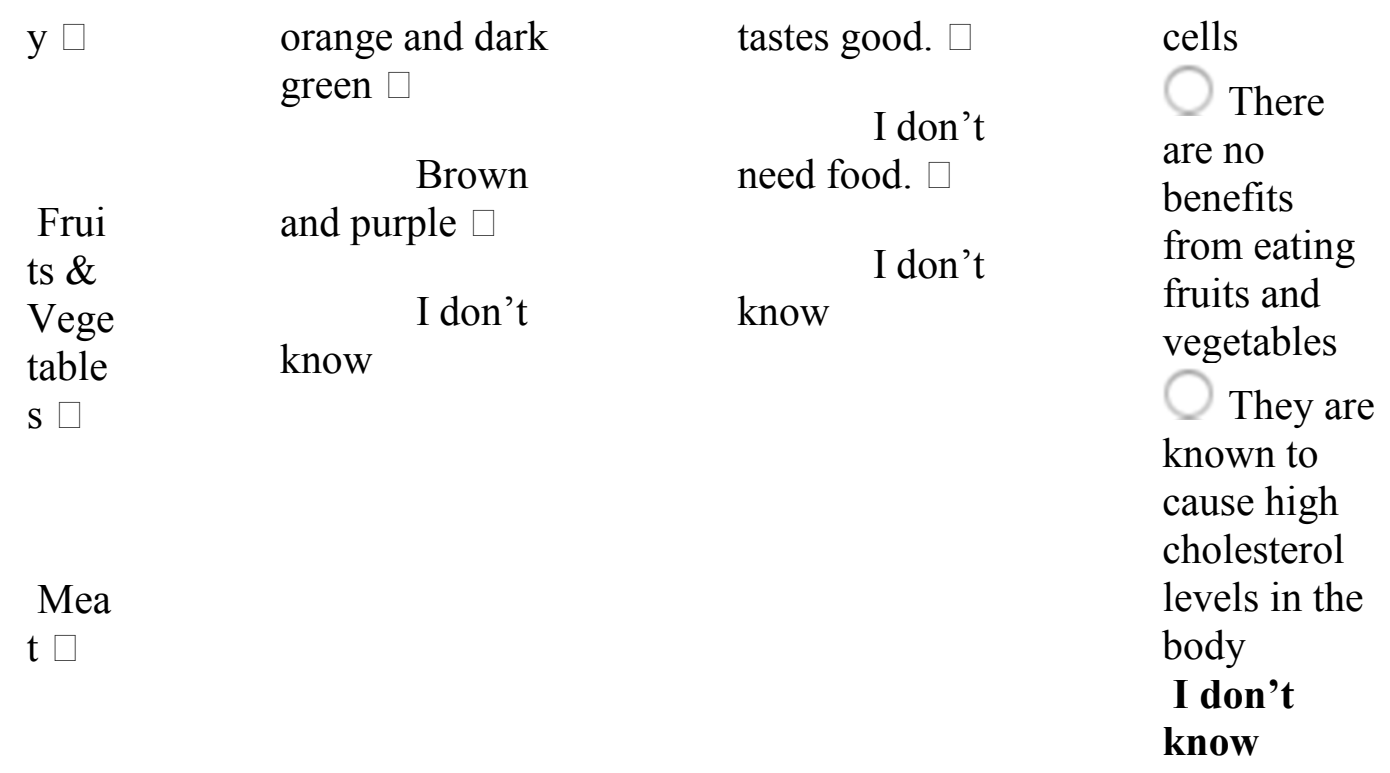

Grai

ns

29. Why do I need to

eat

different

kinds of

foods?

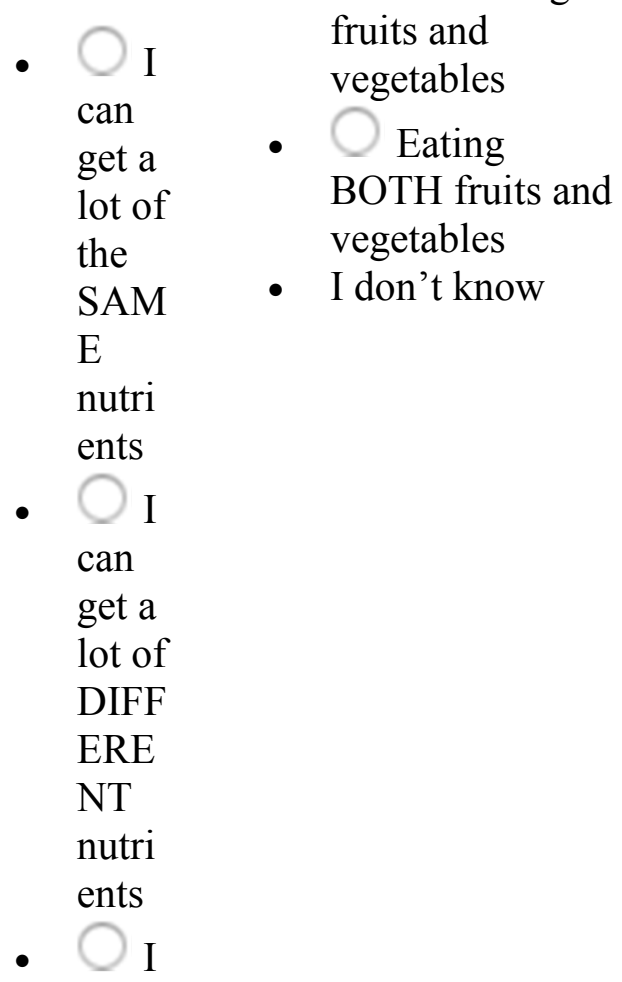

30. Healthy eating is:

- $\bigcirc$ Eating fruits but NOT eating vegetables

- Not eating fruits and BOTH fruits and vegetables

the SAM

E

can

lot of
31. What is the role of fiber from foods?

- $\bigcirc$ To help store nutrients in the body

- To create more interest in the foods we eat

- Elimination of waste from the body

- I don't know
32. How would you best describe the word "microbiota"?

- $\bigcirc$ A place in another country

- $\bigcirc n$ important food group to include in the diet

- $\bigcirc$ A group of microorgani sms that live in the intestines of the human body

- I don't know 


don't
need
to
eat
diffe
rent
kinds
of
food
s
I
don't
kno
w
33. What
helps
improve the
health of
your gut or
your
intestines?
- $\bigcirc$
Fiber
from
food
$\mathrm{s}$
such
as
fruits
and
veget
ables
$\bullet$
Regu
lar
eatin
$\mathrm{g}$
times
ever
$\mathrm{y}$
day

34. The foods that I 35. How likely are eat for meals and you to eat fresh snacks are healthy. fruit instead of (Choose one.) candy? (Choose one.)

Yes, all

of the time $\square \quad$ Not likely

Yes, sometimes $\square \quad$ Likely $\square$

No $\square$

Very Likely 


$$
\begin{aligned}
& \text { Meat } \\
& \text { and } \\
& \text { other } \\
& \text { high } \\
& \text { prote } \\
& \text { in } \\
& \text { food } \\
& \text { s } \\
& \text { I } \\
& \text { don't } \\
& \text { kno } \\
& \text { w }
\end{aligned}
$$

\section{How \\ likely are you to eat fresh vegetables instead of candy? (Choose one.)}

Not likely

Very Likely

$\begin{array}{ll}\begin{array}{ll}\text { 40. How } \\ \text { sure are you } \\ \text { that you can } \\ \text { do the }\end{array} & \begin{array}{l}\text { 41. How sure are you } \\ \text { that you can do the } \\ \text { following: }\end{array} \\ \begin{array}{l}\text { Eat a } \\ \text { grocery setables at the } \\ \text { vegetable } \\ \text { fhat's being }\end{array} & \text { I know I can } \\ \begin{array}{l}\text { served with } \\ \text { my lunch at }\end{array} & \text { I think I can } \square \\ \text { school. } & \text { I'm not sure I can }\end{array}$

37. Which of these is the HEALTHIEST way to eat potatoes?

salad $\square$ Potato
fries $\square$

$$
\begin{aligned}
& \text { Baked } \\
& \text { potato } \square
\end{aligned}
$$

I don't know
Likely $\square$

I'm not sure I can
38. How sure are you that you can do the following: Eat vegetables at dinner.

I know I can

$$
\text { I think I can } \square
$$

I'm not sure I can

I know I can't

42. How sure are you that you can do the following: Eat a fruits that's being served with my lunch at school.

I know I can

I think I can $\square$

I'm not sure I can
39. How sure are you that you can do the following: Eat fruit instead of desert with dinner.

I know I can

I think I can $\square$

I'm not sure I can

I know I can't
43. How sure are you that you can do the following: Ask someone to serve my favorite fruit or vegetable with a meal.

I know I can

I think I can $\square$ 


\begin{tabular}{|c|c|c|c|}
\hline I know I can & I know I can't & I know I can't & I'm not sure I can \\
\hline $\begin{array}{l}\text { I think I } \\
\text { can } \square\end{array}$ & & & I know I can't \\
\hline $\begin{array}{l}\text { I'm not sure } \\
\text { I can }\end{array}$ & & & \\
\hline $\begin{array}{l}\text { I know I } \\
\text { can't }\end{array}$ & & & \\
\hline $\begin{array}{l}\text { 45. If I eat } \\
\text { fruits and } \\
\text { vegetables }\end{array}$ & & & \\
\hline $\begin{array}{l}\text { every day I } \\
\text { will become } \\
\text { stronger. } \\
\text { (Circle one). }\end{array}$ & $\begin{array}{l}\text { 44. If I eat fruits and } \\
\text { vegetables every day my } \\
\text { friends will start eating } \\
\text { them too. }\end{array}$ & $\begin{array}{l}\text { 46. If I eat fruits and } \\
\text { vegetables every day I } \\
\text { will have stronger } \\
\text { eves. }\end{array}$ & $\begin{array}{l}\text { 47. If I eat fruits and } \\
\text { vegetables every } \\
\text { day I will have a } \\
\text { nicer smile }\end{array}$ \\
\hline I disagree & & & I disagree very \\
\hline very much & I disagree very much & I disagree very much & much \\
\hline I disagree a & I disagree a little & I disagree a little & I disagree a little \\
\hline little & I am not sure & I am not sure & I am not sure \\
\hline $\begin{array}{l}\text { I am not } \\
\text { sure }\end{array}$ & I agree & I agree & I agree \\
\hline I agree & I agree very much & I agree very much & I agree very much \\
\hline $\begin{array}{l}\text { I agree very } \\
\text { much }\end{array}$ & & & \\
\hline $\begin{array}{l}\text { 48. If I eat } \\
\text { fruits and } \\
\text { vegetables } \\
\text { every day I } \\
\text { will be }\end{array}$ & $\begin{array}{l}49 \text { If I eat fruits and } \\
\text { vegetables every day I } \\
\text { will think better in class. } \\
\text { I disagree very much }\end{array}$ & $\begin{array}{l}50 . \text { If I eat fruits and } \\
\text { vegetables every day I } \\
\text { will have more energy. } \\
\text { I disagree very much }\end{array}$ & $\begin{array}{l}51 \text { If I eat fruits and } \\
\text { vegetables every } \\
\text { day my family will } \\
\text { be proud of me. }\end{array}$ \\
\hline healthier. & I disagree a little & I disagree a little & $\begin{array}{l}\text { I disagree very } \\
\text { much }\end{array}$ \\
\hline $\begin{array}{l}\text { I disagree } \\
\text { very much }\end{array}$ & I am not sure & I am not sure & I disagree a little \\
\hline I disagree a & I agree & I agree & I am not sure \\
\hline
\end{tabular}




\begin{tabular}{|c|c|c|c|}
\hline little & I agree very much & I agree very much & I agree \\
\hline $\begin{array}{l}\text { I am not } \\
\text { sure }\end{array}$ & & & I agree very much \\
\hline \multicolumn{4}{|l|}{ I agree } \\
\hline \multicolumn{4}{|l|}{$\begin{array}{l}\text { I agree very } \\
\text { much }\end{array}$} \\
\hline $\begin{array}{l}52 . \text { I think I } \\
\text { can ask } \\
\text { someone in } \\
\text { my family to } \\
\text { make my }\end{array}$ & $\begin{array}{l}\text { 53. I think I can ask } \\
\text { someone in my family to } \\
\text { serve my favorite fruit at } \\
\text { dinner. }\end{array}$ & $\begin{array}{l}\text { 54. I think I can eat } 2 \\
\text { or more servings of } \\
\text { fruit or fruit juice each } \\
\text { day. }\end{array}$ & $\begin{array}{l}55 . \text { I think I can eat } \\
3 \text { or more servings } \\
\text { of vegetables each } \\
\text { day. }\end{array}$ \\
\hline favorite & I disagree very much & I disagree very much & $\begin{array}{l}\text { I disagree very } \\
\text { much }\end{array}$ \\
\hline $\begin{array}{l}\text { dish for } \\
\text { dinner. }\end{array}$ & I am not sure & I am not sure & I disagree a little \\
\hline $\begin{array}{l}\text { I disagree } \\
\text { very much }\end{array}$ & I agree & I agree & \\
\hline $\begin{array}{l}\text { I disagree a } \\
\text { little }\end{array}$ & I agree very much & I agree very much & $\begin{array}{l}\text { I agree } \\
\text { I agree very much }\end{array}$ \\
\hline \multicolumn{4}{|l|}{$\begin{array}{l}\text { I am not } \\
\text { sure }\end{array}$} \\
\hline \multicolumn{4}{|l|}{ I agree } \\
\hline $\begin{array}{l}\text { agree very } \\
\text { uch }\end{array}$ & & & \\
\hline
\end{tabular}

Think about everything you ate or drank YESTERDAY. Remember what you had for breakfast, lunch, dinner, after school, and while watching TV, and at bedtime.

Did you eat or drink it yesterday?
53. Apples, bananas, or oranges

54. Applesauce, fruit cocktail

55. Any other fruit, like
O Yes

O No

O Yes

O No

O Yes
How much did you eat?

O $1 / 2$

O $1 \quad$ O 2

O A little

O Some O A lot

O No $\mathbf{O}$ A little O Some O A 
strawberries, grapes

56. French fries, hash

browns, tater tots

O Yes

O No

lot

57. Other potatoes, like

O Yes

O No

O A little

O Some O A lot

mashed or boiled

58. Ketchup or salsa

O Yes

O No

O A little

O Some O A lot

59. Lettuce salad

O Yes

O No

O A little O Some O A lot

61. Tomatoes, including on $\mathbf{O}$ Yes salad

O No

O A little O Some O A lot

62. Green beans or peas

O Yes

O No

O $1 / 4$ tomato $\mathrm{O} \frac{1 / 2}{2}$ tomato $O$ 1 tomato

63. Other vegetables, like 0 Yes corn, carrots, greens, broccoli

64. Vegetable soup, tomato 0 Yes soup, any soup or stew with vegetables in it

65. Chili beans, pinto beans, $\mathrm{O}$ Yes black beans, including in burritos

66. Refried beans

O Yes

O No

O A little O Some O A lot

O No

O A little O Some O A lot

O A little O Some O A lot

O No $\mathbf{O}$ A little O Some O A lot

O No

O A little O Some O A lot 

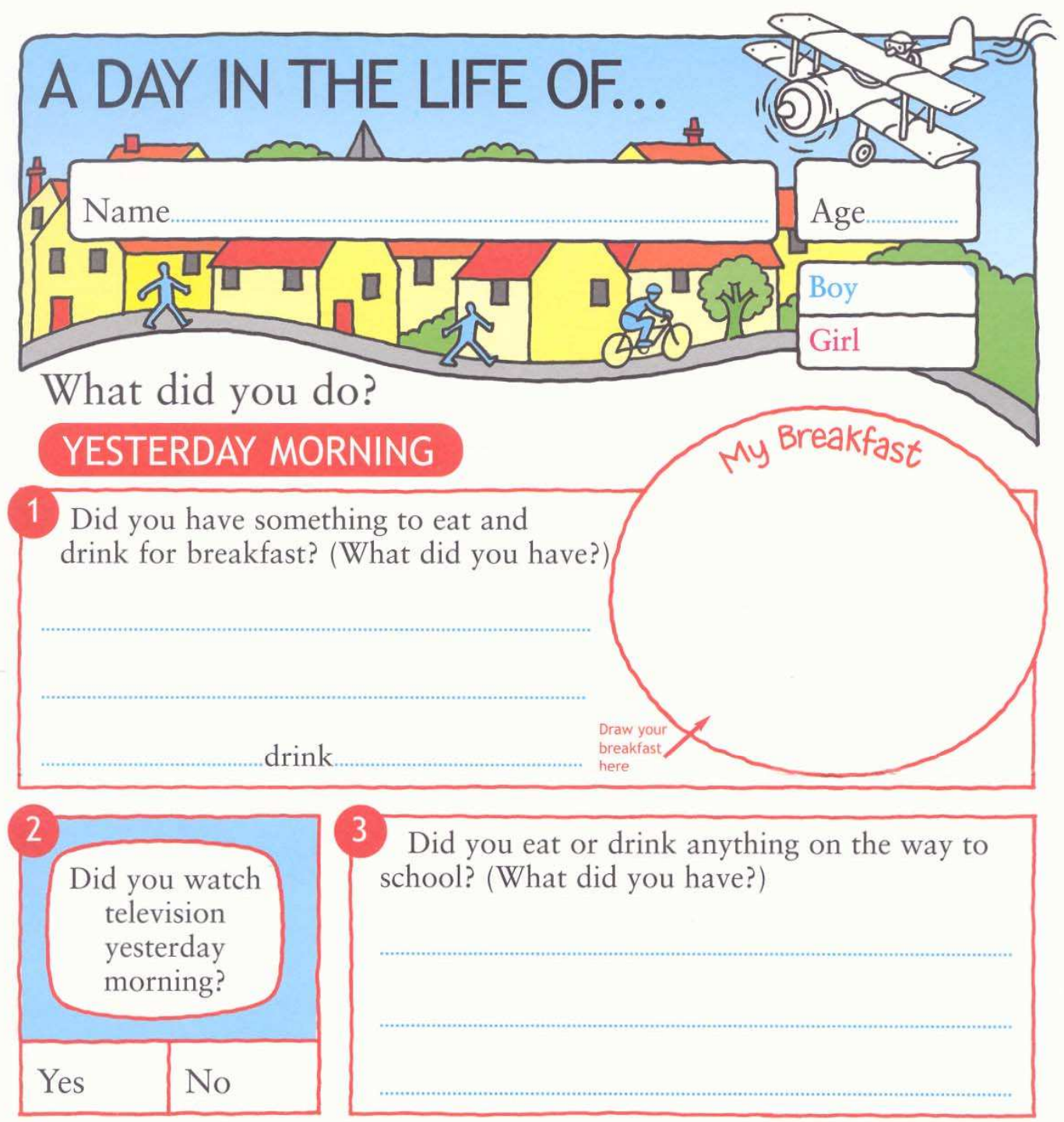

3 Did you eat or drink anything on the way to school? (What did you have?)

4 How did you travel to school yesterday morning?
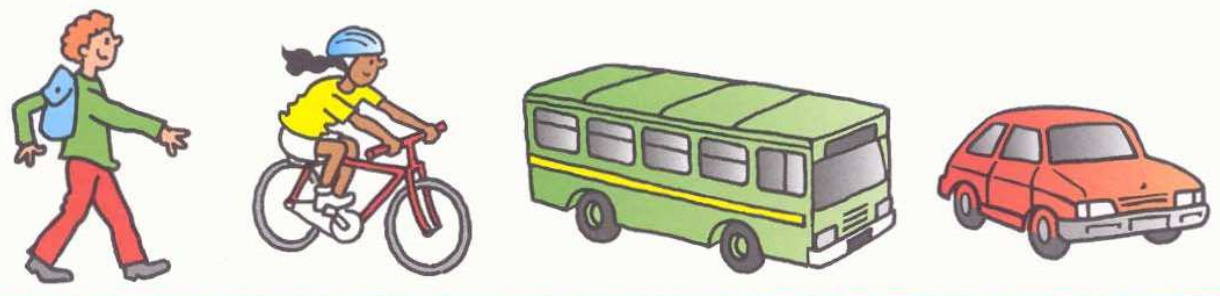

walk

cycle

by bus

by car 


\section{YESTERDAY AT SCHOOL}

5

Did you have anything to eat or drink at morning break?

(What did you have)

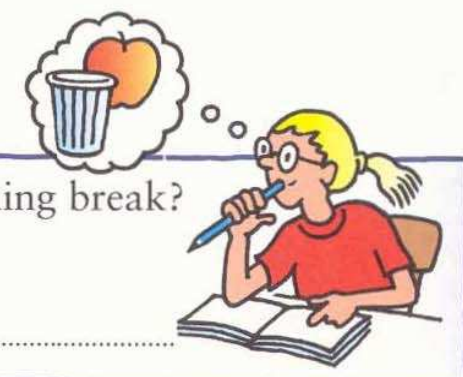

6 What did you do at morning break (interval) yesterday?
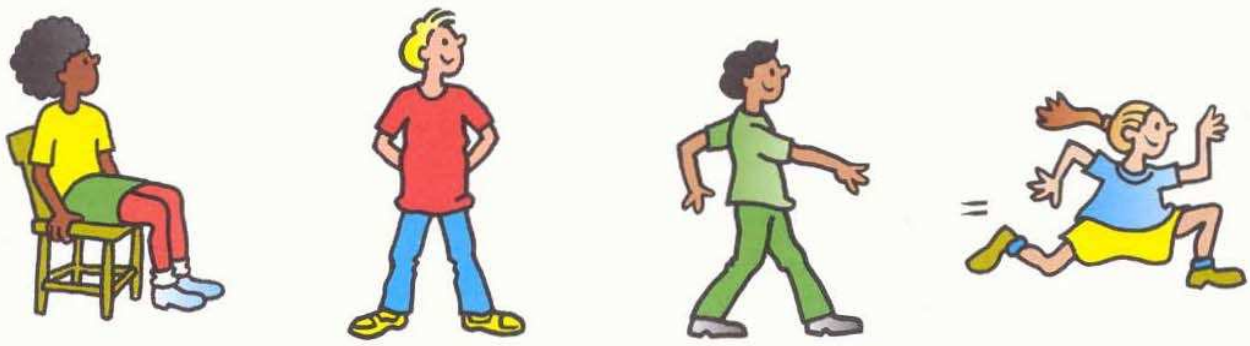

sit around

stand around

\section{walk around}

run around

7 Did you eat and drink anything for lunch yesterday? (What did you have?)

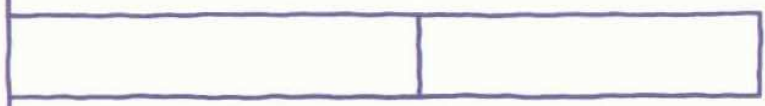

drink

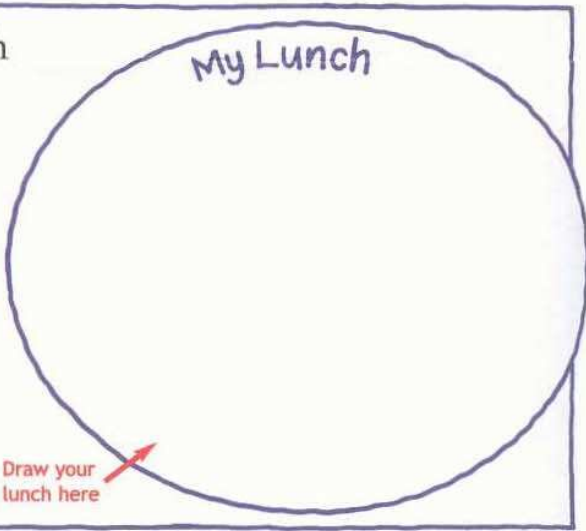

8 What did you do at lunchtime yesterday?

You can color in these activities
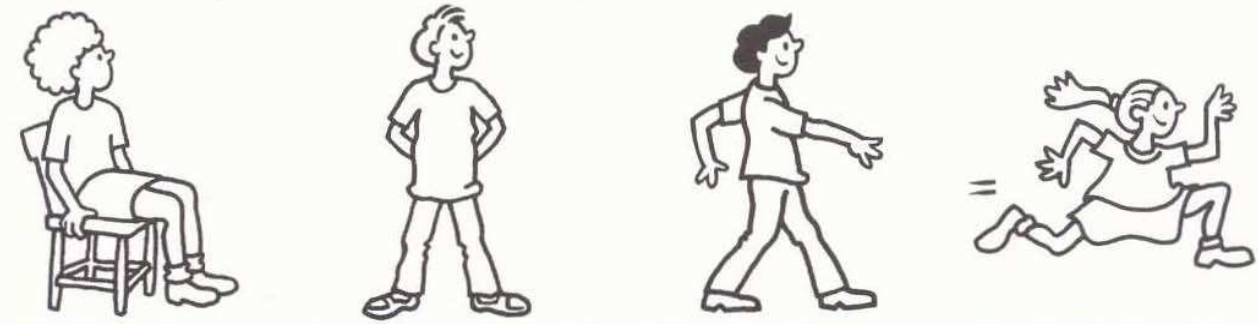

sit around

stand around

walk around

run around 


\section{AFTER SCHOOL}

9

How did you travel home after school or your after school care yesterday?
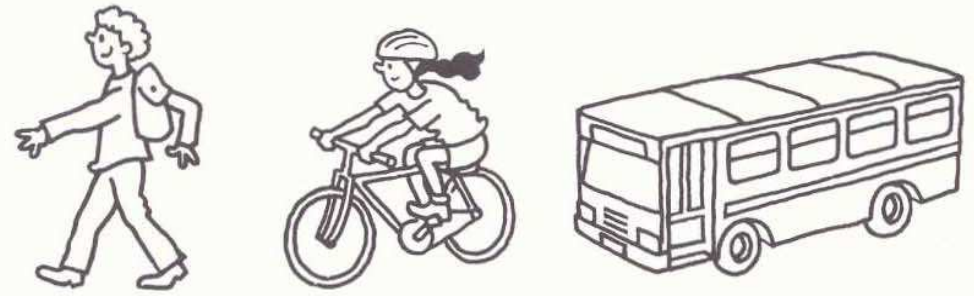

You can color in these drawings

walk

cycle

by bus

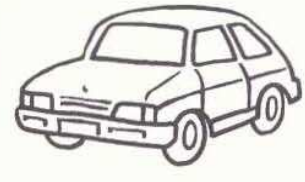

Did you eat or drink anything when you were travelling home? (What did you have)

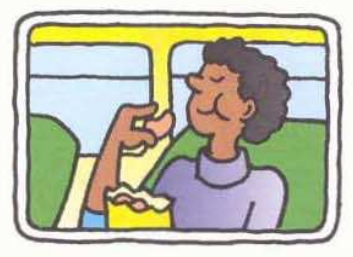

11

After school yesterday, did you:

\begin{tabular}{|l|l|}
\hline go home? \\
\hline go to a club (eg Brownies, Cubs, swimming, football?) \\
\hline go to after school club?
\end{tabular}

Did you have anything to eat, or something to drink between the end of school (apart from the journey) and your evening meal?

(What did you have?) 
AFIER SCHOOL (continuad)

13

Did you play outside yesterday after school? Yes

No
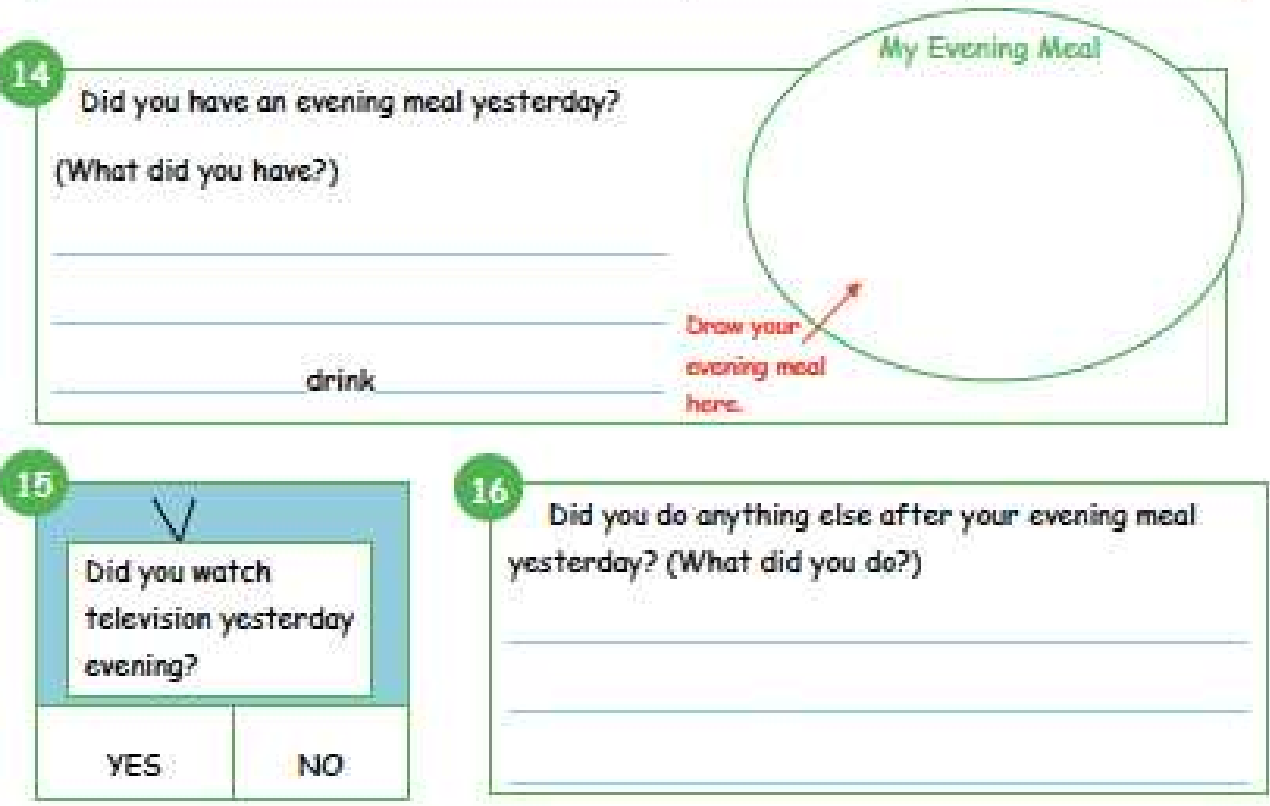

17 Did you have anything else to eat or drink between your evening meal and before you went to bed? (What did you have?)

\section{THANK YOU VERY MUCH!}


Appendix G-3 The Adapted "Eat at America's Table" (EATS) survey (Survey 3)

INSTRUCTIONS: Think about what you usually offered your child to eat in the last month.

Please think about all foods that you offered last month. Include those that were: raw and cooked, eaten as snacks and at meals, $\square$ eaten at home and away from home (restaurants, friends, take-out), and eaten alone and mixed with other foods.

Report how many times per month, week, or day you offered each food, and if your child ate it, and how much they usually had.

If you mark "Never" for a question, follow the "Go to" instruction. Choose the best answer for each question. Mark only one response for each question. 
1. Over the last month, how many times per month, week, or day did you offer to your child drinks that are $\mathbf{1 0 0 \%}$ juice such as orange, apple, grape, or grapefruit juice? Don't count fruit drinks like Kool-Aid, lemonade, Hi-C, cranberry juice drink, Tang, and Twister. Include juice you drank at all mealtimes and between meals.

$\begin{array}{llllllllll}\text { Never } & 1-3 & 1-2 & 3-4 & 5-6 & 1 & 2 & 3 & 4 & 5+\end{array}$

(Go to Times Times Times Times Time Times Times Times Times

Question Last Per Per Per Per Per Per Per Per

2) month week week week day day day day day

1a. Each time you offered this beverage, how often did he/she drink $\mathbf{1 0 0 \%}$ juice, how much did you usually drink?

\begin{tabular}{|c|c|c|c|}
\hline Less than $3 / 4$ cup & $3 / 4$ to $11 / 4$ cup & $1 \frac{1}{4}$ cups to 2 cups & More than 2 cups \\
\hline $\begin{array}{c}\text { (less than } 6 \\
\text { ounces) }\end{array}$ & (6-10 ounces) & (10 o 16 ounces) & $\begin{array}{c}\text { (more than } 16 \\
\text { ounces) }\end{array}$ \\
\hline
\end{tabular}

2. Over the last month, how many times per month, week, or day did you offer your child fruit? Count any kind of fruit-fresh, canned, and frozen. Do not count juices. Include fruit you offered at all mealtimes and for snacks.

\begin{tabular}{|c|c|c|c|c|c|c|c|c|c|}
\hline Never & $1-3$ & $1-2$ & $3-4$ & $5-6$ & 1 & 2 & 3 & 4 & $5+$ \\
\hline (Go to & Times & Times & Times & Times & Time & Times & Times & Times & Times \\
\hline $\begin{array}{c}\text { Question } \\
3)\end{array}$ & $\begin{array}{l}\text { Last } \\
\text { month }\end{array}$ & $\begin{array}{c}\text { Per } \\
\text { week }\end{array}$ & $\begin{array}{c}\text { Per } \\
\text { week }\end{array}$ & $\begin{array}{c}\text { Per } \\
\text { week }\end{array}$ & $\begin{array}{l}\text { Per } \\
\text { day }\end{array}$ & $\begin{array}{l}\text { Per } \\
\text { day }\end{array}$ & $\begin{array}{l}\text { Per } \\
\text { day }\end{array}$ & $\begin{array}{l}\text { Per } \\
\text { day }\end{array}$ & $\begin{array}{l}\text { Per } \\
\text { day }\end{array}$ \\
\hline
\end{tabular}

2a. Each time you offered fruit, how much did they usually eat?

Less than 1 medium fruit 1 medium fruit 2 medium fruits More than 2 
medium fruits

OR

Less than $1 / 2$ cup

About $1 / 2$ cup

About 1 cup More than 1 cup

3. Over the last month, how often did you offer your child lettuce salad (with or without other vegetables)?

\begin{tabular}{|c|c|c|c|c|c|c|c|c|c|}
\hline 0 & 0 & 0 & 0 & 0 & 0 & 0 & 0 & 0 & 0 \\
\hline Never & $1-3$ & $1-2$ & $3-4$ & $5-6$ & 1 & 2 & 3 & 4 & $5+$ \\
\hline (Go to & Times & Times & Times & Times & Time & Times & Times & Times & \\
\hline $\begin{array}{l}\text { Question } \\
4)\end{array}$ & $\begin{array}{l}\text { Last } \\
\text { month }\end{array}$ & Per & Per & Per & er & Per & Per & Per & \\
\hline
\end{tabular}

3a. Each time you offered lettuce salad, how much did they usually eat?

About $1 / 2$ cup About 1 cup

About 2 cups $\square \quad$ More than 2 cups

4. Over the last month, how often did you offer French fries or fried potatoes?

$\begin{array}{cccccccccc}\text { Never } & 1-3 & 1-2 & 3-4 & 5-6 & 1 & 2 & 3 & 4 & 5+ \\ \text { (Go to } & \text { Times } & \text { Times } & \text { Times } & \text { Times } & \text { Time } & \text { Times } & \text { Times } & \text { Times } & \text { Times } \\ \text { Question } & \text { Last } & \text { Per } & \text { Per } & \text { Per } & \text { Per } & \text { Per } & \text { Per } & \text { Per } & \text { Per } \\ \text { 5) } & \text { month } & \text { week } & \text { week } & \text { week } & \text { day } & \text { day } & \text { day } & \text { day } & \text { day }\end{array}$

4a. Each time you offered French fries or fried potatoes, how much did they usually eat?

Small order or less $\quad$ Medium order or less $\quad$ Large order $\quad$ Super size or more 
(About 1 cup or less) (About $1 \frac{1}{2}$ cups) $\quad$ (About 2 cups)

(About 3 cups or more)

5. Over the last month, how often did you offer to your child to eat other white potatoes? Count baked, boiled, and mashed potatoes, potato salad, and white potatoes that were not fried.

\begin{tabular}{|c|c|c|c|c|c|c|c|c|c|}
\hline 0 & 0 & 0 & 0 & 0 & 0 & 0 & 0 & 0 & 0 \\
\hline Never & $1-3$ & $1-2$ & $3-4$ & $5-6$ & 1 & 2 & 3 & 4 & $5+$ \\
\hline (Go to & Times & Times & Times & Times & Time & Times & Times & Times & imes \\
\hline $\begin{array}{l}\text { Question } \\
\text { 6) }\end{array}$ & $\begin{array}{l}\text { Last } \\
\text { month }\end{array}$ & $\begin{array}{c}\text { Per } \\
\text { week }\end{array}$ & $\begin{array}{c}\text { Per } \\
\text { week }\end{array}$ & $\begin{array}{c}\text { Per } \\
\text { week }\end{array}$ & $\begin{array}{l}\text { Per } \\
\text { day }\end{array}$ & $\begin{array}{l}\text { Per } \\
\text { day }\end{array}$ & $\begin{array}{l}\text { Per } \\
\text { day }\end{array}$ & $\begin{array}{l}\text { Per } \\
\text { day }\end{array}$ & ay \\
\hline
\end{tabular}

5a. Each time you offered these potatoes, how much did they usually eat?

1 Small potato or less 1 Medium potato or less 1 Large potato or less 2 medium potatoes

or more

(1/2 cup or less)

(About $1 / 2$ cup - 1 cup)

(About 1 cup-1 1/2 cups)

(About 3 cups or more) 
6. Over the last month, how often did you offer to your child to eat cooked dried beans? Count baked beans, bean soup, refried beans, pork and beans and other bean dishes.

$\begin{array}{cccccccccc}\text { Never } & 1-3 & 1-2 & 3-4 & 5-6 & 1 & 2 & 3 & 4 & 5+ \\ \text { (Go to } & \text { Times } & \text { Times } & \text { Times } & \text { Times } & \text { Time } & \text { Times } & \text { Times } & \text { Times } & \text { Times } \\ \text { Question } & \text { Last } & \text { Per } & \text { Per } & \text { Per } & \text { Per } & \text { Per } & \text { Per } & \text { Per } & \text { Per } \\ 7 \text { 7) } & \text { month } & \text { week } & \text { week } & \text { week } & \text { day } & \text { day } & \text { day } & \text { day } & \text { day }\end{array}$

6a. Each time you offered these beans, how much did they usually eat?

Less than $1 / 2$ cup $\quad 1 / 2$ to 1 cup 1 to $1 \frac{1}{2}$ cups More than $1 \%$ cups

7. Over the last month, how often did you offer other vegetables? DO NOT COUNT: Lettuce salads $\square$ White potatoes $\square$ Cooked dried beans $\square$ Vegetables in mixtures, such as in sandwiches, omelets, casseroles, Mexican dishes, stews, stir-fry, soups, etc.

\section{COUNT:}

Rice $\square$ All other vegetables-raw, cooked, canned, and frozen

$\begin{array}{llllllllll}\text { Never } & 1-3 & 1-2 & 3-4 & 5-6 & 1 & 2 & 3 & 4 & 5+\end{array}$

(Go to Times Times Times Times Time Times Times Times Times

Question Last Per Per Per Per Per Per Per Per

8) month week week week day day day day day

7a. Each of these times that you offered other vegetables, how much did they usually eat?

Less than $1 / 2$ cup $1 / 2$ to 1 cup 1 to 2 cups More than 2 cups 
8. Over the last month, how often did you offer macaroni, rice, pizza and other dishes. with tomato sauce? Include tomato sauce on pasta or

$\begin{array}{cccccccccc}\text { Never } & 1-3 & 1-2 & 3-4 & 5-6 & 1 & 2 & 3 & 4 & 5+ \\ \text { Gro to } & \text { Times } & \text { Times } & \text { Times } & \text { Times } & \text { Time } & \text { Times } & \text { Times } & \text { Times } & \text { Times } \\ \begin{array}{c}\text { Question } \\ 9)\end{array} & \begin{array}{c}\text { Last } \\ \text { month }\end{array} & \begin{array}{c}\text { Per } \\ \text { week }\end{array} & \begin{array}{c}\text { Per } \\ \text { week }\end{array} & \begin{array}{c}\text { Per } \\ \text { week }\end{array} & \begin{array}{c}\text { Per } \\ \text { day }\end{array} & \begin{array}{c}\text { Per } \\ \text { day }\end{array} & \begin{array}{c}\text { Per } \\ \text { day }\end{array} & \begin{array}{c}\text { Per } \\ \text { day }\end{array} & \begin{array}{c}\text { Per } \\ \text { day }\end{array}\end{array}$

8 a. Each time you ate tomato sauce, how much did they usually eat?
About $1 / 4$ cup
About $1 / 2$ cup
About 1 cup
More than 1 cup

9. Over the last month, how often did you $\square$ offer vegetable soup, minestrone soup, and other soups made with vegetables? Include tomato soup, gazpacho, beef with vegetable soup and other soups made with vegetables.

\begin{tabular}{|c|c|c|c|c|c|c|c|c|c|}
\hline Never & $1-3$ & $1-2$ & $3-4$ & $5-6$ & 1 & 2 & 3 & 4 & $5+$ \\
\hline (Go to & Times & Times & Times & Times & Time & Times & Times & Times & Times \\
\hline $\begin{array}{c}\text { Question } \\
10)\end{array}$ & $\begin{array}{l}\text { Last } \\
\text { month }\end{array}$ & $\begin{array}{c}\text { Per } \\
\text { week }\end{array}$ & $\begin{array}{c}\text { Per } \\
\text { week }\end{array}$ & $\begin{array}{c}\text { Per } \\
\text { week }\end{array}$ & $\begin{array}{l}\text { Per } \\
\text { day }\end{array}$ & $\begin{array}{l}\text { Per } \\
\text { day }\end{array}$ & $\begin{array}{l}\text { Per } \\
\text { day }\end{array}$ & $\begin{array}{l}\text { Per } \\
\text { day }\end{array}$ & $\begin{array}{l}\text { Per } \\
\text { day }\end{array}$ \\
\hline
\end{tabular}

9a. Each time you offered vegetable soup, how much did they usually eat?
Less than 1 cup
1 to 2 cups
2 to 3 cups
More than 3 cups

10. Over the last month, how often did you offer your child to eat mixtures that included vegetables? Count such foods as sandwiches, casseroles, stews, stir-fry, omelets, and tacos. 
$\begin{array}{lllllllll}\text { Never } & 1-3 & 1-2 & 3-4 & 5-6 & 1 & 2 & 3 & 4\end{array}$

Times Times Times Times Time Times Times Times Times

Last Per Per Per Per Per Per Per Per month week week week day day day day day

How to Score the Screener

(https://epi.grants.cancer.gov/diet/screeners/fruitveg/scoring/allday.html)

For the All-Day screener, scoring involves a series of operations that are shown below and implemented in the All-Day Screener Pyramid Servings SAS Program and the All-Day Screener MyPyramid Cup Equivalents SAS Program.

1. Express each reported frequency as a daily average. To do this, standardize the midpoint of each frequency category to the number of times per day.

\section{Frequency Response Times Per Day}

Never

0.0

1-3 times per month

0.067

1-2 times per week

0.214

3-4 times per week

0.5

5-6 times per week

0.786

1 time per day

1.0

2 times per day

2.0

3 time per day

4 times per day

4.0

5 or more times per day 5.0

2. Assign fruit and vegetable Pyramid/MyPyramid servings to each portion size category.

1. Using 2005 MyPyramid definitions of fruit and vegetable cup equivalents:

\section{Food}

\begin{tabular}{cllllll} 
& \multicolumn{1}{c}{$\mathbf{1}$} & \multicolumn{2}{c}{$\mathbf{3}$} & \multicolumn{2}{c}{$\mathbf{4}$} \\
Juice & .5 & 1.0 & 1.625 & 2.5 & \\
Fruit (units) & .25 & .5 & 1.0 & 1.5 \\
Fruit (cups) & .25 & .5 & 1.0 & 1.5 \\
Lettuce salad & .25 & .5 & 1.0 & 1.5 \\
French fries & .2 & .5 & .75 & 1.3
\end{tabular}


Food

MyPyramid Cup Equivalents for each Portion Size Category

\begin{tabular}{cccccl} 
Other white & \multicolumn{1}{c}{} & \multicolumn{2}{c}{$\mathbf{3}$} & & $\mathbf{4}$ \\
potatoes & .25 & .75 & 1.2 & 2.0 \\
Dried beans & .25 & .75 & 1.25 & 2.0 \\
Other vegetables & .25 & .75 & 1.5 & 2.25 \\
Tomato sauce & .25 & .5 & 1.0 & 1.5 \\
Vegetable soups & .3 & 1.0 & 1.6 & 2.25
\end{tabular}

2. Using 1992-2004 definition of Pyramid servings:

\section{Food}

\begin{tabular}{cllll} 
& \multicolumn{1}{c}{$\mathbf{1}$} & \multicolumn{2}{c}{$\mathbf{2}$} & \multicolumn{1}{c}{$\mathbf{4}$} \\
Juice & .75 & 1.33 & 2.17 & 3.33 \\
Fruit (units) & .75 & 1.0 & 2.0 & 2.5 \\
Fruit (cups) & .75 & 1.0 & 2.0 & 2.5 \\
Lettuce salad & .5 & 1.0 & 2.0 & 3.0 \\
French fries & 1.25 & 2.3 & 3.1 & 4.8 \\
Other white & .8 & 1.5 & 2.4 & 3.5 \\
potatoes & & 1.5 & 2.5 & 3.5 \\
Dried beans & .75 & 1.5 & 3.0 & 4.5 \\
Other vegetables & .75 & .72 & 1.45 & 1.7 \\
Tomato sauce & .36 & 1.36 & 2.27 & 3.2 \\
Vegetable soups & .75 & &
\end{tabular}

3. Compute the average daily fruit and vegetable Pyramid/MyPyramid servings for each food group by multiplying the average daily frequency (from \#1) by the number of Pyramid/MyPyramid servings for the portion size (as determined in \#2). To estimate the total daily number of

Pyramid/MyPyramid servings, sum across all food groups.

1. not yet been tested.

Note: Question 10 -- "How often over the past month did you eat mixtures that included vegetables?" -- is not included in this

algorithm. This question may be helpful in identifying individuals who eat a lot of their vegetables in mixtures. If amounts of vegetables eaten in mixtures are harder to report, their reports may be somewhat less accurate. However, this supposition has Return to Top 


\section{Earlier Changes in Scoring}

On July 12,2001 , the following changes were made to the calculations used to estimate fruits and vegetables from the screener data:

1. In the calculations to convert the frequency categories to number of times per day:

○ 1-3 times per month was changed from .066 to .067 times per day,

- 3-4 times per week was changed from .499 to .5 times per day, and

- 5-6 times per week was changed from .784 to .786 times per day.

2. In the assignment of fruit and vegetable Pyramid servings to portion size categories:

○ the highest portion size category for juice was changed from 2.5 to 3.33 Pyramid servings and

$\circ \quad$ the highest portion size category for lettuce was changed from 2.5 to 3.0 Pyramid serving. 


\section{APPENDIX G-4: The Adapted Youth Adolescent Food Frequency Questionnaire O \\ - for weekly and monthly food recalls completed by parents about their child.}

1. What is your age?

2. Are you male or female?

3. What is your height?

4. What is your weight?

5. Do you take vitamins (like Flintstones, Centrum, etc) Yes or No 5a. If yes how many times per week:
$1-2$
$3-5$
6-9
10 or more

6. Do you take any other separate vitamin or mineral pills? Yes or No 6a. if yes do you take:

Calcium or Tums Iron Vitamin C Fish Oil Vitamin E

Vitamin D Other please specify 
Think about your eating habits over the past year or so. About how often do you eat each of the following foods? Remember breakfast, lunch, dinner, snacks and eating out. Check one square for each food.

Do you eat any of the following foods? (There are no right or wrong answers).

\begin{tabular}{lllllll} 
Fruits, Vegetables, & Less than & Once a & $2-3$ & $4-6$ & Once & $2+$ a \\
and Grains & 1/WEEK & WEEK & times a & times a & a & DAY \\
\hline
\end{tabular}

Fruit juice, like orange, apple, grape, fresh, frozen or canned. (Not sodas or other drinks)

How often do you eat any fruit, fresh or canned (not counting juice?)

Vegetable juice, like tomato juice, $\mathrm{V}-8$, carrot

Green salad

Potatoes, any kind, including baked, mashed or french fried

Vegetable soup, or stew with vegetables Any other vegetables, including string beans, peas, corn, broccoli or any other kind Fiber cereals like Raisin Bran, Shredded Wheat or Fruit-n-Fiber Beans such as baked beans, pinto, kidney, or lentils (not green beans)

Dark bread such as whole wheat or rye 

1. Cold breakfast cereal (1 bowl)
2. Which cold breakfast cereal do you eat most often?

Never/less than 1 per mont

1-3 bowls per month

1 bowl per week

2-4 bowls per week

5-7 bowls per week

More than 1 bowl per day
Cheerios

Honey Nut Cheerios

Frosted Mini-Wheats

Frosted Flakes

Cinnamon Toast Crunch

Other:

\section{Oatmeal, include instant (1 bowl)}

Never/less than 1 per month

1-3 bowls per month 1 bowl per week 2-4 bowls per week 5-7 bowls per week More than 1 bowl per day

\section{Croissant (1)}

Never/less than 1 per month

1-3 per month $\square$

1 per week $\square$

2-4 per week $\square$

More than 4 per week

\section{Salsa (1 serving)}

Never/less than 1 per month

1-3 times per month

Once per week

2-6 times per week

1 or more times per day

5. White bread, pita bread, include toast - not in sandwiches (1 slice)

Never/less than 1 per month

1 slice per week or less

$\square$ 2-4 slices per week $\square$

5-7 slices per week

2-3 slices per day $\square$

More than 3 slices a day

\section{Cold breakfast cereal (1 bowl)}

Never/less than 1 per month

1-3 bowls per month $\square$

1 bowl per week

2-4 bowls per week

5-7 bowls per week $\square$

More than 1 bowl per day

\section{Oatmeal, include instant (1 bowl)}

Never/less than 1 per month

1-3 bowls per month

1 bowl per week

2-4 bowls per week

5-7 bowls per week

$\square$ More than 1 bowl per day
9. English muffins, bagels, or rolls (1) include breakfast sandwich

Never/less than 1 per month

1-3 per month $\square$

1 per week

$\square$ 2-4 per week 
More than 4 per week

10. White rice (1 serving)

Never/less than 1 per month

1-3 times per month

$\square$ Once per week $\square$

2-4 times per week $\square$

More than 4 times per week

\section{French toast (2 slices)}

Never/less than 1 per month

1-3 times per month $\square$

Once per week

$\square$ 2-4 times per week $\square$

More than 4 times a week
12. Brown rice (1 serving)

Never/less than 1 per month

1-3 times per month

$\square$ Once per week $\square$

2-4 times per week $\square$

More than 4 times per week

15. Corn or flour tortilla—no filling ( $n o t$ tacos or burritos) (2)

Never/less than 1 per month

1-3 per month
2-4 times per week $\square$

More than 4 times per week

1-3 times per month $\square$

Once per week $\square$

14. Pancakes or waffles (2)

Never/less than 1 per month $\square 2-4$ servings per week
More than 4 servings per
week $\square 2-4$ servings per week
More than 4 servings per
week

1 serving per week

2-4 servings per week
More than 4 servings per
week

13. French fries, tater tots,

Never/less than 1 per month

1-3 servings per month $\square$

2-4 per week $\square$

More than 4 per week

\section{Raisins (small pack)}

Never/less than 1 per month $\square$

1-3 times per month $\square$

1 per week

2-4 times per week $\square$
18. Applesauce (1 serving)

Never/less than 1 per month $\square$

1-3 times per month

1 per week

2-4 times per week 
More than 4 times per week More than 4 times per week

\section{Grapes (bunch)}

Never/less than 1 per month $\square$

1-3 times per month $\square$

1 per week

2-4 times per week $\square$

More than 4 times per week

\section{Watermelon ( 1 wedge)}

Never/less than once per month

$1-3$ times per month $\square$

Once per week $\square$

More than once per week

\section{Grapefruit (1/2)}

Never/less than 1

$1-3$ per month $\square$

1 per week $\square$

2-6 per week $\square$

1 or more per day

\section{Bananas (1)}

Never/less than 1 per month $\square$

1-3 times per month $\square$

1 per week

2-4 times per week $\square$

More than 4 times per week

\section{Blueberries (1 serving)}

Never/less than 1 per month

1-3 times per month $\square$

Once per week $\square$

2-4 times per week $\square$

More than 4 times per week

26. Pineapple (1 serving)

Never/less than $1 \square$ per month

1-3 times per month

Once per week $\square$

More than once per week
More than 4 times per week

\section{Apples (1)}

Never/less than 1 per month

1-3 per month $\square$

1 per week $\square$

2-6 per week $\square$

1 or more per day

24. Apple juice and other $100 \%$ fruit juices (1 glass)

Never/less than 1 per month

1-3 glasses per month

1 glass per week $\square$

2-6 glasses per week

1 glass per day

More than 1 glass per day

\section{Cantaloupe, melon} (1 wedge)

Never/less than once per month

1-3 times per month

$\square$ Once per week $\square$

More than once per week 
28. Strawberries (1 serving)

Never/less than 1 per month

1-3 times per month $\square$

Once per week $\square$

2-4 times per week

More than 4 times per week

\section{Orange juice (1 glass) 30. Oranges (1)}

Never/less than 1 month Never/less than 1

1-3 glasses per month $\square \quad 1-3$ per month $\square$

1 glass per week $\square \quad 1$ per week

2-6 glasses per week $\quad \square$ 2-6 per week $\square$

1 glass per day $\square \quad 1$ or more per day

More than 1 per day

31. Peaches, plums, apricots (1)

Never/less than 1 per month

1-3 per month $\square$

1 per week

2-4 per week

More than 4 per week

\section{Tomatoes (2 slices)}

Never/less than 1 per month 1-3 per month $\square$

1 per week $\square$

2-6 per week

1 or more per day
33. Beans or lentilsinclude baked beans ( 1 serving)

Never/less than 1 per month

Once per week or less

2-6 times per week

More than 4 times per week
34. Tomato juice (1 small glass)

Never/less than 1 per month

1-3 per month $\square$

\section{Broccoli (1 serving)}

Never/less than 1 per month

1-3 times per month

Once per week
36. Mixed vegetables (1 serving)

Never/less than 1 per month

1-3 times per month $\square$ 
1 per week $\square$

2-6 per week

1 or more per day

37. Yams/sweet potatoes (medium or 1 serving)

Never/less than 1 per month

1-3 times per month

Once per week $\square$

2-4 times per week

More than 4 times per week

$\square$ 2-4 times per week $\square$

More than 4 times per week

$$
\text { More than } 4 \text { times per week }
$$

38. Celery ( 2 or 3 sticks)

Never/less than 1 per month

1-3 times per month

$\square$ Once per week

$\square$ 2-4 times per week $\square$

More than 4 times a week
More than 4 times per week

Once per week $\square$

2-4 times per week

39. Potato salad (small serving)

Never/less than 1 per month

1-3 times per month

Once per week $\square$

More than once per week

\section{V8 Fusion $\square$ (1 small glass)}

Never/less than 1 per month

1-3 per month $\square$

1 per week $\square$

2-6 per week

1 or more per day

\section{Cauliflower (1 serving)}

Never/less than 1 per month

1-3 times per month $\square$

Once per week

2-4 times per week

More than 4 times per week

\section{Cabbage (1 serving)}

Never/less than 1 per month

1-3 times per month

Once per week $\square$

More than once per week

\section{Zucchini, summer squash, eggplant (1 serving)}

Never/less than 1 per month 1-3 times per month $\square$

\section{Lettuce/tossed salad (1 serving)}

Never/less than 1 per month 1-3 times per month $\square$
45. Pasta salad (small serving)

Never/less than 1 per month

1-3 times per month 
Once per week $\square$

2-4 times per week

More than 4 times per week 1 or more per day

\section{Green beans ( 1} serving)

Never/less than 1 per month

1-3 times per month $\square$

Once per week $\square$

2-4 times per week

More than 4 times per week

\section{Carrots, cooked (1 serving)}

Never/less than 1 per month

1-3 times per month

Once per week $\square$

2-4 times per week

More than 4 times per week

\section{Peas (1 serving)}

Never/less than 1 per month

1-3 times per month

Once per week $\square$

2-4 times per week
Once per week $\square$

2-6 times per week

More than once per week

\section{Corn (1 serving)}

Never/less than 1 per month

1-3 times per month $\square$

Once per week $\square$

2-4 times per week $\square$

More than 4 times per week

\section{Coleslaw (1 serving)}

Never/less than 1 per month

1-3 times per month

Once per week $\square$

More than once per week

53. Green/red/yellow peppers ( 3 slices)

Never/less than 1 per month

1-3 times per month

Once per week
48. Collard greens/kale/ cooked spinach (1 serving)

Never/less than 1 per month

1-3 times per month

Once per week

$\square 2-4$ times per week

More than 4 times per week

\section{Okra (1 serving)}

Never/less than 1 per month

1-3 times per month

Once per week $\square$

More than once per week

54. Carrots, raw ( $1 / 2$ carrot or 2-4 baby carrots)

Never/less than 1 per month

1-3 times per month $\square$ 
More than 4 times per week $\quad 2-4$ times per week

More than 4 times per week

More than 4 times per week
Once per week

$\square 2-4$ times per week 


\section{APPENDIX G-5: DATA COLLECTION FORMS}

\begin{tabular}{|c|c|c|c|c|c|c|c|}
\hline $\begin{array}{l}\text { STUDY } \\
\text { ID }\end{array}$ & $\begin{array}{l}\text { BASe } \\
\text { HEIGHT }\end{array}$ & $\begin{array}{l}\text { POST } \\
\text { HEIGHT }\end{array}$ & $\begin{array}{l}\text { BASE } \\
\text { WEIGHT }\end{array}$ & $\begin{array}{l}\text { POST } \\
\text { WEIGHT }\end{array}$ & $\begin{array}{l}\text { BASE } \\
\text { BP }\end{array}$ & $\begin{array}{l}\text { POST } \\
\text { BP }\end{array}$ & AGE \\
\hline & & & & & & & \\
\hline & & & & & & & \\
\hline & & & & & & & \\
\hline & & & & & & & \\
\hline & & & & & & & \\
\hline & & & & & & & \\
\hline & & & & & & & \\
\hline & & & & & & & \\
\hline & & & & & & & \\
\hline & & & & & & & \\
\hline & & & & & & & \\
\hline & & & & & & & \\
\hline & & & & & & & \\
\hline & & & & & & & \\
\hline & & & & & & & \\
\hline & & & & & & & \\
\hline & & & & & & & \\
\hline & & & & & & & \\
\hline & & & & & & & \\
\hline & & & & & & & \\
\hline & & & & & & & \\
\hline & & & & & & & \\
\hline & & & & & & & \\
\hline & & & & & & & \\
\hline & & & & & & & \\
\hline & & & & & & & \\
\hline & & & & & & & \\
\hline & & & & & & & \\
\hline & & & & & & & \\
\hline & & & & & & & \\
\hline & & & & & & & \\
\hline & & & & & & & \\
\hline
\end{tabular}




\title{
APPENDIX H: Scores for knowledge, attitude, self-efficacy/perception surveys
}

\author{
Kuowledge and Attitudes Survey Scoring Procedure \\ Updated Decomber 18, 2013
}

The scoring procedures described here spply to soth pre-and post-test sdministration scoring To evaluste pre/post chenge, calculate the difference in construct scores by subtracting the pre-test score from the post-test scare (esch section separately)

\section{Scorfing proceduris}

students' responses from the KA survey were categorized into different scores.

1) Onowledge: There are seventeen questions in this section. The questions focus on m sterial typically covered in fam to school progrsms. Score the questions sccordingly:

- Comect responses = score 1

- incorred answers = score o fincluding the response f don'f mow, wher that is : response option)

Scores can range from 0 to maximum of 17 . To determine s students knowledge score, add the sum of all cuestions in this section $(022-37,040)$.

$$
\text { Knowledge }=\text { L(comect respoizes, }, 022-37,940)
$$

2) Attitudes: There are twenty questions in this section. Six questions (a1-3, $Q 11-13$ ] ask how much a student likes $F N$ and how much a student fites now F/N. Score the responses as:

\begin{tabular}{|l|l|l|l|}
\hline a lot & a ittie & notwerf much & not at all \\
\hline 4 & 3 & 2 & 1 \\
\hline
\end{tabular}

Tweve questions (q4-9, $Q 14-19)$ ask student' willingness to try a fN in a variety or situations. Score remponces as:

\begin{tabular}{|l|l|l|l|}
\hline defiritey & probebly & probsbly not & definitely nat \\
\hline 4 & 3 & 2 & 1 \\
\hline
\end{tabular}

Finally, two questions ( $(10, \mathrm{Q} 20$ ) ask how many times s student fad tried a new F/V since the tart al the school yebr. Soore responses as:

\begin{tabular}{|l|l|l|l|l|}
\hline Never & 1 time & 2 times & 3 times & At lest 4 times \\
\hline 1 & 2 & 3 & 4 & 9 \\
\hline
\end{tabular}

scores can range from 20 to a maximum of 32 . To determine a sudent's Attitudes rare, add the sum of all the questions in this section $[\mathrm{q} 1-20]$.

$$
\text { Attindes }=\text { E(scored rasponses, } Q 1-20)
$$

3) Ferception/self-etticacy: There are six questions in this section. Two coestions (o38-39) ack: sudents' perception of their own diets - whether the foods they est are healthy. Score reponses as:

\begin{tabular}{|l|l|l|l|}
\hline Q38 & Yes, all the tine & Yes, sometimes & no \\
\hline Q39 & Yery firel & Likely & Nat libely \\
\hline Score & 2 & 1 & 0 \\
\hline
\end{tabular}

Four addional quetions (0a0-83) ask students to reflect on their sbility to make hesithy dhoices est vegetables at diner, eat fruit ingtead of desert at dinner, est a vegetable being served st sthool lunch; and eat a fruit beirg served st school hunch. Soore resonses as: 


\begin{tabular}{|l|l|l|l|}
\hline I know I can & I think I can & P'm not sure I can & I know I can't \\
\hline 4 & 3 & 2 & 1 \\
\hline
\end{tabular}

Scores can range from 4 to s maximum of 20. To determine a student's Perception/self-Efficacy score, add the sum of all the questions in this section [Q38-39. Q80-83).

Perception/self efficacy $=\Sigma($ scored responses, $Q 38-39, Q 00-83)$

4) Exposure: There are 26 questione in this section (Q41-66, parts a). Esch question asks if a student has tried a particular fruit or vegetable. Each question includes a photograph to aid with food recognition. Score responses as:

\begin{tabular}{|l|l|}
\hline Yes & No \\
\hline 1 & 0 \\
\hline
\end{tabular}

Scores can range from 0 to a maximum of 26 . To determine a student's Exposure score, add the sum of all the questions in this section $\{Q 41-66$, parts a $\})$.

$$
\text { Exposure }=\sum \text { (yes responses, Q41- 66, parts a) }
$$

The student's response to the Exposure question branches to either a Liking (response yes) or Willingness (response no) follow-up question for woch $F / V$.

9] Liking: (Q41-56, parts b) Where students ancwered 'yes" to part o (above), students are asked whether they liked the fruit/vegetable item that they reported tasting. Score responses as:

\begin{tabular}{|l|l|}
\hline Yes & No \\
\hline 1 & 0 \\
\hline
\end{tabular}

To determine a student's Liking score, divide the sum of the responces for the questions in this section (Q41-66, parts b) by the student's Exposure score i= the total number of truits/vegetables the student tried); finaliy, express it as a percentage by multiplining by 100. Scores can range from 0 to s maximum of 100 .

$$
\text { Liking }=\frac{\sum(\text { yes reponses, } Q 41-66, \text { parts b) }}{\text { Exposine score }} \times 100
$$

6) Willingness: See footnote for old scoring. New scoring (os of May 2013) imwolved asking all students whether they would try the 26 specific $F / \mathrm{N}$ (Q41-66; parts c).

\begin{tabular}{|l|l|l|}
\hline Yes & Maybe & No \\
\hline 2 & 1 & 0 \\
\hline
\end{tabular}

To determine a student's Willingress score, add the sum of all the questions in this section (Q41-65, parts c) and divide oy 2 . Scores can range from 0 to a masimum of 26.

$$
\text { Willingress }=\frac{\sum \text { (scored responses, } Q 41-66, \text { parts c) }}{2}
$$

\footnotetext{
${ }^{7}$ Old scoring: Among the previously not-tasted $F / N$, students were asked whether they would try it (ves, score:2, mopoe, score $=1, n o$, score $=0$. Additionally, for each $F / N$ students reported trying and iking, they received a score of 2 (because it was assurred that they would try a F/N they had previousty tried and iked). The colective sum of all responses was divided by two. Scores can range from 0 to 20.
} 
7] FV Screener: There are 13 questions in this section $\{Q 67-69\}$. For s variety of groups of fruit or vegetable items, students sre asked to report ( $s$ ) whether they ate the item(s) in the past day. and (o) if so, how much (o relative amount). Score section (a) responses as:

\begin{tabular}{|l|l|}
\hline Yes & No \\
\hline 1 & 0 \\
\hline
\end{tabular}

Section (b) response scoring strotegies are included within each subsection below. The Evaluation Team suggests scoring ond sssessing the informstion from this FV screener sS follows:

- Calculate the percent of students who report having eating any food from the subsections (each separately). For example, as\% of students reported eating any fruit in pre-test, and $100 \%$ of students reported eating any truit ot post-test; or $25 \%$ of students reported eating any legumes in the pre-test, and $30 \%$ of students reported eating any legumes in the post-test.) Compare the percent of students with a subsection total score groater than $O$ to the percent of students with a subsection totol scove of exoctly 0 .

- For esch subsection, calculste the average relative a mount students reported consuming and relate it back to the terms used for that subsection,

\section{FVScreenes-Subsectioun:}

b. Fruit (Q67-69)

i. Ate fruit at $a b=$ score $>0$, parts $a$

ii. Reistive amount: Score responses as:

\begin{tabular}{|l|l|l|l|}
\hline Response & $-11^{*}$ & $-1^{*}$ & $2^{*}$ \\
\hline Q67 & 0.5 & 1 & 2 \\
\hline Response & "A little" & -Some & "-A lot" \\
\hline Q68-69 & 0.5 & 1 & 2 \\
\hline
\end{tabular}

To determine a student's relative consumption of fruits, sdd the responses in this subsection and divide by 3.

b. Potatoes $(\mathrm{Q} 70-71)$ :

i. Ate potatoes at all $=$ score $>0$, parts a

ii. Relotive amount: Score responses as:

\begin{tabular}{|l|l|l|l|}
\hline Response & "A little" & "Some" & "A lot" \\
\hline $070-71$ & 1 & 2 & 3 \\
\hline
\end{tabular}

To determine a student's relative consumption of fruits, sdd the responses in this subsection and divide by 2 .

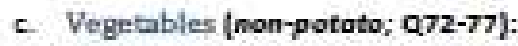

i. Ate vegetabies at all $=$ score 20 , parts $a$

ii. Relotive amount Score responses as:

\begin{tabular}{|l|l|l|l|}
\hline Response & "A little" & - Some" & -A lot" \\
\hline Q72-73, 75-77 & 1 & 2 & 3 \\
\hline Response & $-1 / 4$ tomato" & -4 tomato" & -1 tomato" \\
\hline Q74 & 0.25 & 0.5 & 1 \\
\hline
\end{tabular}

To determine a student's relative consumption of fruits, sdd the responses in this subsection and divide by 5 .

d. Legames:

i. Ate legumes at ail $=$ score $>0$, ports 0

ii. Relative smount Score responses as:

\begin{tabular}{|l|l|l|l|}
\hline Response & "A littie" & "Some" & "A lot" \\
\hline Q78-79 & 1 & 2 & 3 \\
\hline
\end{tabular}

To determine a student's relative consumption of fruits, odd the responses in this subsection and divide by 2 . 


\section{Appendix I: Statistics}

Table I-1: Descriptive statistics from SPSS for each group including age, gender, height, weight, body mass index

levels, z-scores, and blood pressure.

\section{I-1 BASELINE DESCRIPTIVE STATISTICS}

\begin{tabular}{|l|c|c|c|c|c|c|c|}
\hline & N & Min & Maximum & Mean & Std. Error & $\begin{array}{c}\text { Stnd Deviation } \\
\text { Statistic }\end{array}$ & $\begin{array}{c}\text { Variance } \\
\text { Statistic }\end{array}$ \\
\hline Group & 29 & 1 & 2 & 1.45 & 0.094 & 0.506 & 0.256 \\
\hline Age & 29 & 11 & 12 & 11.83 & 0.071 & 0.384 & 0.148 \\
\hline Gender & 29 & 1 & 2 & 1.48 & 0.094 & 0.509 & 0.259 \\
\hline Height & 29 & 142 & 171 & 155.1214 & 1.2927 & 6.9614 & 48.461 \\
\hline Weight & 29 & 33.18 & 76.36 & 47.0066 & 1.74002 & 9.3703 & 87.802 \\
\hline BMI & 29 & 15.63 & 26.27 & 19.4683 & 0.59315 & 3.1942 & 10.203 \\
\hline Baseline Score & 29 & -1 & 2 & 0.3621 & 0.17352 & 0.93443 & 0.873 \\
\hline Pre systolic & 29 & 81 & 119 & 102.3793 & 1.72594 & 9.29444 & 86.387 \\
\hline Pre diastolic & 29 & 51 & 79 & 63.4828 & 1.30046 & 7.00317 & 49.044 \\
\hline $\begin{array}{l}\text { Valid N (List } \\
\text { Wise) }\end{array}$ & 29 & & & & & & \\
\hline
\end{tabular}

\begin{tabular}{|c|c|c|c|c|}
\hline \multicolumn{5}{|c|}{ I-1 GROUP AND GENDER } \\
\hline \multicolumn{5}{|l|}{ Count } \\
\hline & & \multicolumn{2}{|c|}{ Gender } & \multirow{2}{*}{ Total } \\
\hline & & Male & Female & \\
\hline \multirow{2}{*}{ Group } & Intervention & 8 & 8 & 16 \\
\hline & Control & 7 & 6 & 13 \\
\hline \multicolumn{2}{|l|}{ Total } & 15 & 14 & 29 \\
\hline
\end{tabular}




\begin{tabular}{|c|c|c|c|c|}
\hline \multicolumn{5}{|c|}{ GROUP AND ETHNICITY } \\
\hline \multicolumn{5}{|l|}{ Count } \\
\hline & & \multicolumn{3}{|c|}{ RACE } \\
\hline & & Caucasian & $\begin{array}{c}\text { American } \\
\text { Indian }\end{array}$ & Other \\
\hline \multirow{2}{*}{ GROUP } & Intervention & 13 & 1 & 0 \\
\hline & Control & 11 & 0 & 2 \\
\hline \multicolumn{2}{|l|}{ Total } & 24 & 1 & 2 \\
\hline
\end{tabular}

\begin{tabular}{|l|r|r|r|r|r|r|r|r|}
\hline \multicolumn{1}{|l|}{ I-1 POST STUDY DESCRIPTIVE STATISTICS } \\
\hline & $\mathrm{N}$ & Range & Min & Max & Mean & $\begin{array}{c}\text { Stnd } \\
\text { Error }\end{array}$ & $\begin{array}{c}\text { Std. } \\
\text { Dev. }\end{array}$ & Variance \\
\hline Group & 29 & 1 & 1 & 2 & 1.45 & 0.09 & 0.51 & 0.26 \\
\hline Age & 29 & 1 & 11 & 12 & 11.83 & 0.07 & 0.38 & 0.15 \\
\hline Gender & 29 & 1 & 1 & 2 & 1.48 & 0.09 & 0.51 & 0.26 \\
\hline Race & 27 & 6 & 0 & 6 & 3.07 & 0.24 & 1.24 & 1.53 \\
\hline $\begin{array}{l}\text { Post } \\
\text { height }\end{array}$ & 29 & 31.25 & 142.13 & 173.38 & 155.91 & 1.36 & 7.30 & 53.35 \\
\hline $\begin{array}{l}\text { Post } \\
\text { weight }\end{array}$ & 29 & 44.55 & 33.18 & 77.73 & 47.80 & 1.77 & 9.53 & 90.75 \\
\hline $\begin{array}{l}\text { Post } \\
\text { BMI }\end{array}$ & 29 & 10.48 & 15.54 & 26.02 & 19.64 & 0.57 & 3.10 & 9.59 \\
\hline $\begin{array}{l}\text { Post } \\
\text { systolic }\end{array}$ & 29 & 39 & 77 & 116 & 98.76 & 1.86 & 10.03 & 100.54 \\
\hline $\begin{array}{l}\text { Post } \\
\text { diastolic }\end{array}$ & 29 & 34 & 47 & 81 & 63.69 & 1.82 & 9.81 & 96.29 \\
\hline $\begin{array}{l}\text { Valid N } \\
\text { (listwise) }\end{array}$ & 27 & & & & & & & \\
\hline
\end{tabular}


Table I-2:Intervention group statistical analysis with paired t-tests for baseline and post study measures.

\begin{tabular}{|c|c|c|c|c|c|}
\hline \multicolumn{6}{|c|}{ I-2 INTERVENTION GROUP: Paired Samples Statistics } \\
\hline \multicolumn{2}{|r|}{ MEASURE } & \multirow{2}{*}{$\begin{array}{l}\text { Mean } \\
155.58\end{array}$} & \multirow{2}{*}{$\begin{array}{r}\text { N } \\
16\end{array}$} & \multirow{2}{*}{$\begin{array}{c}\begin{array}{c}\text { Std. } \\
\text { Deviation }\end{array} \\
7.30\end{array}$} & \multirow{2}{*}{$\begin{array}{c}\begin{array}{c}\text { Std. } \\
\text { Error } \\
\text { Mean }\end{array} \\
1.82\end{array}$} \\
\hline \multirow{2}{*}{ Pair 1} & Height & & & & \\
\hline & Post Height & 156.67 & 16 & 7.71 & 1.92 \\
\hline \multirow{2}{*}{ Pair 2} & Weight & 47.29 & 16 & 10.87 & 2.71 \\
\hline & Post Weight & 48.12 & 16 & 11.14 & 2.78 \\
\hline \multirow{2}{*}{ Pair 3} & BMI & 19.39 & 16 & 3.38 & 0.84 \\
\hline & Post BMI & 19.49 & 16 & 3.26 & 0.81 \\
\hline \multirow{2}{*}{ Pair 4} & Base Z-Score & 0.37 & 16 & 0.95 & 0.23 \\
\hline & Post Z-Score & 0.53 & 16 & 0.84 & 0.21 \\
\hline \multirow{2}{*}{ Pair 5} & Pre Systolic & 104.25 & 16 & 9.49 & 2.37 \\
\hline & Post Systolic & 99.37 & 16 & 11.91 & 2.97 \\
\hline \multirow{2}{*}{ Pair 6} & Pre Diastolic & 62.62 & 16 & 8.08 & 2.02 \\
\hline & Post Diastolic & 64.68 & 16 & 10.32 & 2.58 \\
\hline \multirow{2}{*}{ Pair 7} & Base Fruit Obs* & 1.00 & 16 & 0.54 & 0.13 \\
\hline & Post Fruit Obs & 1.29 & 16 & 0.92 & 0.23 \\
\hline \multirow{2}{*}{ Pair 8} & Base Vegetable Obs & 0.33 & 16 & 0.59 & 0.14 \\
\hline & Post Vegetable Obs & 0.03 & 16 & 0.12 & 0.03 \\
\hline \multirow{2}{*}{ Pair 9} & Base Both F/V Obs & 1.33 & 16 & 0.96 & 0.24 \\
\hline & Post Both Obs & 1.32 & 16 & 0.96 & 0.24 \\
\hline \multirow{2}{*}{$\begin{array}{l}\text { Pair } \\
10\end{array}$} & Base Total Consumption Obs & 1.14 & 16 & 0.64 & 0.16 \\
\hline & Post Total Consumption Obs & 1.05 & 16 & 0.83 & 0.20 \\
\hline
\end{tabular}

*OBS$=$ Observation 


\begin{tabular}{|c|c|c|c|c|c|c|c|c|c|}
\hline \multicolumn{10}{|c|}{ I-2 PAIRED SAMPLES TEST } \\
\hline & & \multicolumn{5}{|c|}{ Paired Differences } & \multirow{3}{*}{$\mathrm{t}$} & \multirow{3}{*}{$\mathrm{df}$} & \multirow{3}{*}{$\begin{array}{l}\text { Sig } \\
.2- \\
\text { tail } \\
\text { ed }\end{array}$} \\
\hline & & \multirow[t]{2}{*}{ Mean } & \multirow[t]{2}{*}{$\mathrm{sd}^{*}$} & \multirow{2}{*}{$\begin{array}{l}\text { Std. } \\
\text { Error } \\
\text { Mean }\end{array}$} & \multicolumn{2}{|c|}{$\begin{array}{l}95 \% \text { Confidence } \\
\text { Interval of the } \\
\text { Difference }\end{array}$} & & & \\
\hline & & & & & Lower & Upper & & & \\
\hline $\begin{array}{l}\text { Pair } \\
1\end{array}$ & $\begin{array}{l}\text { Height- Post } \\
\text { Height }\end{array}$ & -1.09 & 0.92 & 0.22 & -1.58 & -0.60 & -4.76 & 15 & 0 \\
\hline $\begin{array}{l}\text { Pair } \\
2\end{array}$ & $\begin{array}{l}\text { Weight - Post } \\
\text { Weight }\end{array}$ & -0.82 & 0.67 & 0.16 & -1.18 & -0.46 & -4.86 & 15 & 0 \\
\hline $\begin{array}{l}\text { Pair } \\
3 \\
\end{array}$ & BMI- Post BMI & -0.10 & 0.24 & 0.06 & -0.23 & 0.03 & -1.60 & 15 & $\begin{array}{r}0.1 \\
2 \\
\end{array}$ \\
\hline $\begin{array}{l}\text { Pair } \\
4\end{array}$ & $\begin{array}{l}\text { Base Z Score - } \\
\text { Post Z Score }\end{array}$ & -0.16 & 0.30 & 0.07 & -0.31 & 0.01 & -2.07 & 15 & $\begin{array}{r}0.0 \\
6\end{array}$ \\
\hline $\begin{array}{l}\text { Pair } \\
5\end{array}$ & $\begin{array}{l}\text { Pre systolic - } \\
\text { Post systolic }\end{array}$ & 4.87 & 7.88 & 1.97 & 0.67 & 9.07 & 2.47 & 15 & $\begin{array}{r}0.0 \\
3\end{array}$ \\
\hline $\begin{array}{l}\text { Pair } \\
6\end{array}$ & $\begin{array}{l}\text { Pre diastolic - } \\
\text { Post diastolic }\end{array}$ & -2.06 & 11.72 & 2.93 & -8.30 & 4.18 & -0.70 & 15 & $\begin{array}{r}0.4 \\
9\end{array}$ \\
\hline $\begin{array}{l}\text { Pair } \\
7\end{array}$ & $\begin{array}{l}\text { Base fruit obs - } \\
\text { Post fruit obs }\end{array}$ & -0.29 & 0.72 & 0.18 & -0.68 & 0.09 & -1.6 & 15 & $\begin{array}{r}0.1 \\
3\end{array}$ \\
\hline $\begin{array}{l}\text { Pair } \\
8\end{array}$ & $\begin{array}{l}\text { Base veg obs - } \\
\text { Post veg obs }\end{array}$ & 0.30 & 0.59 & 0.15 & -0.01 & 0.62 & 2.03 & 15 & $\begin{array}{r}0.0 \\
6 \\
\end{array}$ \\
\hline $\begin{array}{l}\text { Pair } \\
9\end{array}$ & $\begin{array}{l}\text { Base both obs - } \\
\text { Post both obs }\end{array}$ & 0.01 & 0.67 & 0.17 & -0.35 & 0.37 & 0.06 & 15 & $\begin{array}{r}0.9 \\
5\end{array}$ \\
\hline $\begin{array}{l}\text { Pair } \\
10\end{array}$ & $\begin{array}{l}\text { Base Total } \\
\text { Consumption } \\
\text { Obs - Post Total } \\
\text { Consumption } \\
\text {.Obs } \\
\end{array}$ & 0.08 & 0.88 & 0.22 & -0.38 & 0.55 & 0.39 & 15 & $\begin{array}{r}0.6 \\
9\end{array}$ \\
\hline
\end{tabular}




\begin{tabular}{|c|c|c|c|c|c|}
\hline \multicolumn{6}{|c|}{$\begin{array}{l}\text { I-2 RELATIVE AMOUNTS AND DAY IN THE LIFE QUESTIONNAIRE Paired Samples } \\
\text { Statistics }\end{array}$} \\
\hline & & Mean & $\mathrm{N}$ & $\begin{array}{c}\text { Std. } \\
\text { Deviation }\end{array}$ & Std. Error Mean \\
\hline \multirow{2}{*}{$\begin{array}{l}\text { Pair } \\
1\end{array}$} & Fruit Relative Amt* & 1.25 & 15 & 0.82 & 0.21 \\
\hline & Post Fruit Relative Amt & 1.05 & 15 & 0.50 & 0.12 \\
\hline \multirow{2}{*}{$\begin{array}{l}\text { Pair } \\
2 \\
\end{array}$} & Potato Relative Amt & 0.50 & 10 & 0.70 & 0.22 \\
\hline & Post Potato Relative Amt & 0.45 & 10 & 0.76 & 0.24 \\
\hline \multirow{2}{*}{$\begin{array}{l}\text { Pair } \\
3\end{array}$} & Vegetable Relative Amt & 0.59 & 14 & 0.39 & 0.10 \\
\hline & Post Veg Relative Amt & 0.88 & 14 & 0.40 & 0.10 \\
\hline \multirow{2}{*}{$\begin{array}{l}\text { Pair } \\
4\end{array}$} & Legumes Relative Amt & 0.33 & 12 & 0.61 & 0.17 \\
\hline & Post Legume Relative Amt & 0.58 & 12 & 0.84 & 0.24 \\
\hline \multirow{2}{*}{$\begin{array}{l}\text { Pair } \\
5\end{array}$} & Baseline Total Relative Amt & 1.73 & 16 & 1.19 & 0.29 \\
\hline & Post Total Relative Amt & 2.64 & 16 & 1.62 & 0.40 \\
\hline \multirow{2}{*}{$\begin{array}{l}\text { Pair } \\
6\end{array}$} & Survey 2 Score & 2.62 & 8 & 1.84 & 0.65 \\
\hline & Post Survey 2 Score & 1.12 & 8 & 1.12 & 0.39 \\
\hline
\end{tabular}


I-2 Intervention group paired t-tests for relative amounts and Day in the Life Questionnaire (DILQ); baseline and post study

\begin{tabular}{|c|c|c|c|c|c|c|c|c|c|}
\hline \multicolumn{10}{|c|}{$\begin{array}{l}\text { I-2 RELATIVE AMOUNT AND DAY IN THE LIFE QUESTIONNAIRE: } \\
\text { PAIRED SAMPLES T-TEST }\end{array}$} \\
\hline & \multirow{3}{*}{ GROUP } & \multicolumn{5}{|c|}{ Paired Differences } & \multirow{3}{*}{$\mathrm{t}$} & \multirow{3}{*}{ df } & \multirow{3}{*}{$\begin{array}{l}\text { Sig. } \\
2- \\
\text { taile } \\
\text { d }\end{array}$} \\
\hline & & \multirow[t]{2}{*}{ Mean } & \multirow[t]{2}{*}{$\begin{array}{l}\text { Std. } \\
\text { Dev. }\end{array}$} & \multirow{2}{*}{$\begin{array}{l}\text { Std. } \\
\text { Error } \\
\text { Mean }\end{array}$} & \multicolumn{2}{|c|}{$\begin{array}{l}95 \% \text { Confidence } \\
\text { Interval of the } \\
\text { Difference }\end{array}$} & & & \\
\hline & & & & & Lower & Upper & & & \\
\hline $\begin{array}{l}\text { Pair } \\
1\end{array}$ & $\begin{array}{l}\text { Fruit Relative } \\
\text { Amt*- } \\
\text { Post Fruit } \\
\text { Relative Amt }\end{array}$ & 0.20 & 0.75 & 0.19 & -0.21 & 0.62 & 1.03 & 14 & 0.31 \\
\hline $\begin{array}{l}\text { Pair } \\
2\end{array}$ & $\begin{array}{l}\text { Potato Relative } \\
\text { Amt - } \\
\text { Post Potato } \\
\text { Relative Amt }\end{array}$ & 0.05 & 1.03 & 0.33 & -0.69 & 0.79 & 0.15 & 9 & 0.88 \\
\hline $\begin{array}{l}\text { Pair } \\
3\end{array}$ & $\begin{array}{l}\text { Vegetable } \\
\text { Relative Amt - } \\
\text { Post Vegetable } \\
\text { Relative Amt }\end{array}$ & -0.29 & 0.31 & 0.08 & -0.46 & -0.12 & -3.48 & 13 & 0.01 \\
\hline $\begin{array}{l}\text { Pair } \\
4\end{array}$ & $\begin{array}{l}\text { Legumes } \\
\text { Relative Amt - } \\
\text { Post Legume } \\
\text { Relative Amt }\end{array}$ & -0.25 & 0.66 & 0.19 & -0.67 & 0.17 & -1.32 & 11 & 0.21 \\
\hline $\begin{array}{l}\text { Pair } \\
5\end{array}$ & $\begin{array}{l}\text { Base Total } \\
\text { Relative Amt - } \\
\text { Post Total } \\
\text { Relative Amt }\end{array}$ & -0.91 & 1.50 & 0.38 & -1.71 & -0.11 & -2.42 & 15 & 0.03 \\
\hline $\begin{array}{l}\text { Pair } \\
6\end{array}$ & $\begin{array}{l}\text { Survey2score - } \\
\text { Postsurvey2 } \\
\text { score }\end{array}$ & 1.50 & 2 & 0.71 & -0.17 & 3.17 & 2.12 & 7 & 0.07 \\
\hline
\end{tabular}

* Amt=Amount 


\section{I-2 INTERVENTION GROUP MEAL OBSERVATION STATISTICS}

\begin{tabular}{|l|r|r|r|}
\hline \multicolumn{1}{|c|}{ I-2 INTERVENTION GROUP LUNCH OBSERVATIONS DESCRIPTIVE } \\
STATISTICS \\
\hline
\end{tabular}

*OBS=OBSERVATION

\section{I-2 INTERVENTION GROUP MEAL OBSERVATION: CORRELATIONS}

\begin{tabular}{|c|c|c|c|c|c|}
\hline & & $\begin{array}{c}\text { Base } \\
\text { Both } \\
\text { F/V Obs }\end{array}$ & $\begin{array}{c}\text { Base Total } \\
\text { Consumption } \\
\text { Obs }\end{array}$ & $\begin{array}{c}\text { Post } \\
\text { Both } \\
\text { F/V Obs }\end{array}$ & $\begin{array}{l}\text { Post Total } \\
\text { Consumpti } \\
\text { on Obs }\end{array}$ \\
\hline \multirow{3}{*}{ Base Both Obs } & $\begin{array}{l}\text { Pearson } \\
\text { Correlation }\end{array}$ & 1 & 0.373 & $.754^{* *}$ & $.807^{* *}$ \\
\hline & Sig. (2-tailed) & & 0.155 & 0.001 & 0 \\
\hline & $\mathrm{N}$ & 16 & 16 & 16 & 16 \\
\hline \multirow{3}{*}{$\begin{array}{l}\text { Base Total } \\
\text { Consumption } \\
\text { Obs }\end{array}$} & $\begin{array}{l}\text { Pearson } \\
\text { Correlation }\end{array}$ & 0.373 & 1 & 0.257 & 0.3 \\
\hline & Sig. (2-tailed) & 0.155 & & 0.336 & 0.258 \\
\hline & $\mathrm{N}$ & 16 & 16 & 16 & 16 \\
\hline \multirow{3}{*}{$\begin{array}{l}\text { Post Both F/V } \\
\text { Obs }\end{array}$} & $\begin{array}{l}\text { Pearson } \\
\text { Correlation }\end{array}$ & $.754^{* *}$ & 0.257 & 1 & $.654^{* *}$ \\
\hline & Sig. (2-tailed) & 0.001 & 0.336 & & 0.006 \\
\hline & $\mathrm{N}$ & 16 & 16 & 16 & 16 \\
\hline \multirow{3}{*}{$\begin{array}{l}\text { Post Total } \\
\text { Consumption } \\
\text { Obs }\end{array}$} & $\begin{array}{l}\text { Pearson } \\
\text { Correlation }\end{array}$ & $.807^{* *}$ & 0.3 & $.654^{* *}$ & 1 \\
\hline & Sig. (2-tailed) & 0 & 0.258 & 0.006 & \\
\hline & $\mathrm{N}$ & 16 & 16 & 16 & 16 \\
\hline
\end{tabular}

**. Correlation is significant at the 0.01 level (2-tailed). 


\begin{tabular}{|c|c|c|c|c|c|}
\hline \multicolumn{6}{|c|}{ I-2 INTERVENTION CORRELATIONS } \\
\hline & & $\begin{array}{l}\text { Base } \\
\text { Both } \\
\text { Obs }\end{array}$ & $\begin{array}{l}\text { Base Total } \\
\text { Consumption } \\
\text { Obs }\end{array}$ & $\begin{array}{c}\text { Post } \\
\text { Both Obs }\end{array}$ & $\begin{array}{l}\text { Post Total } \\
\text { Consumptio } \\
\text { n Obs }\end{array}$ \\
\hline \multirow{3}{*}{ Base Both Obs } & $\begin{array}{l}\text { Pearson } \\
\text { Correlation }\end{array}$ & 1.00 & 0.37 & $.754^{* *}$ & $.807^{* *}$ \\
\hline & $\begin{array}{l}\text { Sig. (2- } \\
\text { tailed) }\end{array}$ & & 0.16 & 0.00 & 0.00 \\
\hline & $\mathrm{N}$ & 16.00 & 16.00 & 16.00 & 16.00 \\
\hline \multirow{3}{*}{$\begin{array}{l}\text { Base Total } \\
\text { Consumption } \\
\text { Obs }\end{array}$} & $\begin{array}{l}\text { Pearson } \\
\text { Correlation }\end{array}$ & 0.37 & 1.00 & 0.26 & 0.30 \\
\hline & $\begin{array}{l}\text { Sig. (2- } \\
\text { tailed) }\end{array}$ & 0.16 & & 0.34 & 0.26 \\
\hline & $\mathrm{N}$ & 16.00 & 16.00 & 16.00 & 16.00 \\
\hline \multirow{3}{*}{ Post Both Obs } & $\begin{array}{l}\text { Pearson } \\
\text { Correlation }\end{array}$ & $.754^{* *}$ & 0.26 & 1.00 & $.654^{* *}$ \\
\hline & $\begin{array}{l}\text { Sig. (2- } \\
\text { tailed) }\end{array}$ & 0.00 & 0.34 & & 0.01 \\
\hline & $\mathrm{N}$ & 16.00 & 16.00 & 16.00 & 16.00 \\
\hline \multirow{3}{*}{$\begin{array}{l}\text { Post Total } \\
\text { Consumption } \\
\text { Obs }\end{array}$} & $\begin{array}{l}\text { Pearson } \\
\text { Correlation }\end{array}$ & $.807^{* *}$ & 0.30 & $.654^{* *}$ & 1.00 \\
\hline & $\begin{array}{l}\text { Sig. (2- } \\
\text { tailed) }\end{array}$ & 0.00 & 0.26 & 0.01 & \\
\hline & $\mathrm{N}$ & 16.00 & 16.00 & 16.00 & 16.00 \\
\hline
\end{tabular}


Table I-3: Control Group statistical analysis with paired t-tests for baseline and post study measures

\begin{tabular}{|c|c|c|c|c|c|}
\hline \multicolumn{6}{|c|}{$\begin{array}{l}\text { I-3 CONTROL GROUP: PAIRED T-TESTS: PERSONAL DATA, MEAL } \\
\text { OBSERVATIONS }\end{array}$} \\
\hline & & Mean & $\mathrm{N}$ & $\begin{array}{c}\text { Std. } \\
\text { Deviation }\end{array}$ & $\begin{array}{l}\text { Std. } \\
\text { Error } \\
\text { Mean }\end{array}$ \\
\hline \multirow{2}{*}{$\begin{array}{l}\text { Pair } \\
1\end{array}$} & Height & 154.55 & 13 & 6.77 & 1.88 \\
\hline & Post Height & 154.99 & 13 & 6.95 & 1.93 \\
\hline \multirow{2}{*}{$\begin{array}{l}\text { Pair } \\
2\end{array}$} & Weight & 46.65 & 13 & 7.54 & 2.09 \\
\hline & Post Weight & 47.41 & 13 & 7.50 & 2.08 \\
\hline \multirow{2}{*}{$\begin{array}{l}\text { Pair } \\
3\end{array}$} & $\mathrm{BMI}$ & 19.55 & 13 & 3.08 & 0.85 \\
\hline & Post BMI & 19.81 & 13 & 3.00 & 0.83 \\
\hline \multirow{2}{*}{$\begin{array}{l}\text { Pair } \\
4\end{array}$} & Baseline Z Score & 0.35 & 13 & 0.94 & 0.26 \\
\hline & Post Z Score & 0.62 & 13 & 0.85 & 0.23 \\
\hline \multirow{2}{*}{$\begin{array}{l}\text { Pair } \\
5\end{array}$} & Base Systolic & 100.08 & 13 & 8.85 & 2.46 \\
\hline & Post Systolic & 98 & 13 & 7.49 & 2.08 \\
\hline \multirow{2}{*}{$\begin{array}{l}\text { Pair } \\
6\end{array}$} & Base Diastolic & 64.54 & 13 & 5.53 & 1.53 \\
\hline & Post Diastolic & 62.46 & 13 & 9.40 & 2.60 \\
\hline \multirow{2}{*}{$\begin{array}{l}\text { Pair } \\
7\end{array}$} & Base Fruit Obs* & 1.11 & 13 & 0.46 & 0.13 \\
\hline & Post Fruit Obs & 1.02 & 13 & 0.65 & 0.18 \\
\hline \multirow{2}{*}{$\begin{array}{l}\text { Pair } \\
8\end{array}$} & Base Veg Obs & 0.21 & 13 & 0.30 & 0.08 \\
\hline & Post Veg Obs & 0.08 & 13 & 0.28 & 0.08 \\
\hline \multirow{2}{*}{$\begin{array}{l}\text { Pair } \\
9\end{array}$} & Base Both Obs & 1.26 & 13 & 0.47 & 0.13 \\
\hline & Post Both Obs & 1.10 & 13 & 0.76 & 0.210 \\
\hline \multirow{2}{*}{$\begin{array}{l}\text { Pair } \\
10\end{array}$} & Base Total Consumption Obs & 1.14 & 13 & 0.51 & 0.14 \\
\hline & Post Total Consumption Obs & 0.83 & 13 & 0.79 & 0.22 \\
\hline \multirow{2}{*}{$\begin{array}{l}\text { Pair } \\
11\end{array}$} & Base Attitude & 68.38 & 13 & 11.03 & 3.06 \\
\hline & Post Attitude & 68 & 13 & 9.26 & 2.57 \\
\hline \multirow{2}{*}{$\begin{array}{l}\text { Pair } \\
12\end{array}$} & Base Knowledge & 7.38 & 13 & 1.33 & 0.37 \\
\hline & Post Knowledge & 7.77 & 13 & 1.88 & 0.52 \\
\hline \multirow{2}{*}{$\begin{array}{l}\text { Pair } \\
13\end{array}$} & Base Perception/Self-Efficacy & 25.69 & 13 & 2.98 & 0.82 \\
\hline & Post Perception/Self-Efficacy & 25.38 & 13 & 2.72 & 0.75 \\
\hline
\end{tabular}

*OBS=OBSERVTION 


\section{I-3 CONTROL GROUP PAIRED T-TESTS: RELATIVE AMOUNTS AND DILQ}

\begin{tabular}{|c|c|c|c|c|c|}
\hline & I-3 CONT & GROUF & $\overline{\text { PAII }}$ & ED T-TEST & \\
\hline REL & IVE AMOUNT & D DAY & TH & LIFE QUE & TIONNAIRE \\
\hline & & Mean & $\mathrm{N}$ & $\begin{array}{c}\text { Std. } \\
\text { Deviation }\end{array}$ & Std. Error Mean \\
\hline & Fruit relative amt & 0.72 & 13 & 0.31 & 0.09 \\
\hline Pair I & Post fruit relative & 0.94 & 13 & 0.36 & 0.10 \\
\hline & $\begin{array}{l}\text { Potato relative } \\
\text { amt }\end{array}$ & 0.27 & 11 & 0.41 & 0.12 \\
\hline Pair 2 & $\begin{array}{l}\text { Post potato } \\
\text { relative }\end{array}$ & 0.68 & 11 & 0.68 & 0.21 \\
\hline Pair 3 & $\begin{array}{l}\text { Vegetable } \\
\text { relative amt }\end{array}$ & 0.60 & 13 & 0.43 & 0.12 \\
\hline & Post veg relative & 0.67 & 13 & 0.51 & 0.14 \\
\hline Doir 1 & $\begin{array}{l}\text { Legumes relative } \\
\text { amt }\end{array}$ & 0.15 & 13 & 0.32 & 0.09 \\
\hline Palr 4 & $\begin{array}{l}\text { Post legume } \\
\text { relative }\end{array}$ & 0.35 & 13 & 0.69 & 0.19 \\
\hline & $\begin{array}{l}\text { Pre Total } \\
\text { Relative amt }\end{array}$ & 1.70 & 13 & 0.91 & 0.25 \\
\hline Pair 5 & $\begin{array}{l}\text { Post Total } \\
\text { Relative amt }\end{array}$ & 2.28 & 13 & 1.34 & 0.37 \\
\hline & Survey 2 score & 2.17 & 12 & 1.40 & 0.41 \\
\hline Pair 6 & $\begin{array}{l}\text { Post survey } 2 \mathrm{~s} \\
\text { core }\end{array}$ & 1.58 & 12 & 1.16 & 0.34 \\
\hline
\end{tabular}




\begin{tabular}{|c|c|c|c|c|c|c|c|c|}
\hline \multicolumn{9}{|c|}{ I-3 CONTROL GROUP: PAIRED SAMPLES T-TEST } \\
\hline & & \multicolumn{5}{|c|}{ Paired Differences } & \multirow{3}{*}{$\mathrm{t}$} & \multirow{3}{*}{ df } \\
\hline & & \multirow[t]{2}{*}{ Mean } & \multirow[t]{2}{*}{$\mathrm{SD}^{*}$} & \multirow{2}{*}{$\begin{array}{l}\text { Std. } \\
\text { Error } \\
\text { Mean }\end{array}$} & \multicolumn{2}{|c|}{$\begin{array}{l}95 \% \text { Confidence } \\
\text { Interval of the } \\
\text { Difference }\end{array}$} & & \\
\hline & & & & & Lower & Upper & & \\
\hline Pair 1 & $\begin{array}{l}\text { Height - } \\
\text { Post Height }\end{array}$ & -0.43 & 0.80 & 0.22 & -0.92 & 0.05 & -1.9 & 12 \\
\hline Pair 2 & $\begin{array}{l}\text { Weight - } \\
\text { Post Weight }\end{array}$ & -0.76 & 0.78 & 0.21 & -1.23 & -0.28 & -3.5 & 12 \\
\hline Pair 3 & BMI - Post BMI & -0.25 & 0.42 & 0.11 & -0.50 & 0.002 & -2.15 & 12 \\
\hline Pair 4 & $\begin{array}{l}\text { Baseline Z Score - } \\
\text { Post Z Score }\end{array}$ & -0.26 & 0.25 & 0.07 & -0.42 & -0.11 & -3.74 & 12 \\
\hline Pair 5 & $\begin{array}{l}\text { Base Systolic - } \\
\text { Post Systolic }\end{array}$ & 2.07 & 7.99 & 2.21 & -2.75 & 6.90 & 0.93 & 12 \\
\hline Pair 6 & $\begin{array}{l}\text { Base Diastolic } \\
\text { Post Diastolic }\end{array}$ & 2.07 & 12.22 & 3.39 & -5.30 & 9.46 & 0.61 & 12 \\
\hline Pair 7 & $\begin{array}{l}\text { Base Fruit Obs* - } \\
\text { Post Fruit Obs }\end{array}$ & 0.09 & 0.44 & 0.12 & -0.17 & 0.36 & 0.77 & 12 \\
\hline Pair 8 & $\begin{array}{l}\text { Base veg obs - } \\
\text { Post veg obs }\end{array}$ & 0.13 & 0.34 & 0.09 & -0.07 & 0.34 & 1.38 & 12 \\
\hline Pair 9 & $\begin{array}{l}\text { Base Both obs - } \\
\text { Post Both obs }\end{array}$ & 0.16 & 0.44 & 0.12 & -0.10 & 0.43 & 1.34 & 12 \\
\hline $\begin{array}{l}\text { Pair } \\
10\end{array}$ & $\begin{array}{l}\text { Base Total } \\
\text { Consumption - } \\
\text { Post Total } \\
\text { Consumption } \\
\end{array}$ & 0.31 & 0.74 & 0.20 & -0.13 & 0.75 & 1.51 & 12 \\
\hline $\begin{array}{l}\text { Pair } \\
11 \\
\end{array}$ & $\begin{array}{l}\text { Base Attitude- } \\
\text { Post Attitude }\end{array}$ & 0.38 & 7.63 & 2.11 & -4.22 & 4.99 & 0.18 & 12 \\
\hline $\begin{array}{l}\text { Pair } \\
12\end{array}$ & $\begin{array}{l}\text { Base Knowledge - } \\
\text { Post Knowledge }\end{array}$ & -0.384 & 1.85 & 0.51 & -1.50 & 0.73 & -0.75 & 12 \\
\hline $\begin{array}{l}\text { Pair } \\
13\end{array}$ & $\begin{array}{l}\text { Base Percept/Self } \\
\text { efficacy - } \\
\text { Post Percept/Self } \\
\text { efficacy }\end{array}$ & 0.307 & 2.28 & 0.63 & -1.074 & 1.68 & 0.485 & 12 \\
\hline
\end{tabular}

*SD=STANDARD DEVIATION 


\begin{tabular}{|c|c|c|c|c|c|c|c|c|}
\hline \multicolumn{9}{|c|}{$\begin{array}{c}\text { I-3 CONTROL GROUP: PAIRED SAMPLES T-TEST } \\
\text { FOR RELATIVE AMOUNTS AND DILQ }\end{array}$} \\
\hline & & \multicolumn{5}{|c|}{ Paired Differences } & \multirow{3}{*}{$\mathrm{t}$} & \multirow{3}{*}{$\mathrm{df}$} \\
\hline & & \multirow[t]{2}{*}{ Mean } & \multirow[t]{2}{*}{$\begin{array}{l}\text { Std. } \\
\text { Deviation }\end{array}$} & \multirow{2}{*}{$\begin{array}{l}\text { Std. } \\
\text { Error } \\
\text { Mean }\end{array}$} & \multicolumn{2}{|c|}{$\begin{array}{l}95 \% \text { Confidence } \\
\text { Interval of the } \\
\text { Difference }\end{array}$} & & \\
\hline & & & & & Lower & Upper & & \\
\hline $\begin{array}{l}\text { Pair } \\
14\end{array}$ & $\begin{array}{l}\text { Fruit Relative } \\
\text { Amt - Post } \\
\text { Fruit Relative }\end{array}$ & -0.22 & 0.27 & 0.07 & -0.39 & -0.06 & -2.98 & 12 \\
\hline $\begin{array}{l}\text { Pair } \\
15\end{array}$ & $\begin{array}{l}\text { Potato Relative } \\
\text { Amt - Post } \\
\text { Potato Relative }\end{array}$ & -0.40 & 0.83 & 0.25 & -0.96 & 0.14 & -1.63 & 10 \\
\hline $\begin{array}{l}\text { Pair } \\
16\end{array}$ & $\begin{array}{l}\text { Vegetable } \\
\text { Relative Amt - } \\
\text { Post Veg } \\
\text { Relative }\end{array}$ & -0.07 & 0.35 & 0.09 & -0.28 & 0.14 & -0.74 & 12 \\
\hline $\begin{array}{l}\text { Pair } \\
17\end{array}$ & $\begin{array}{l}\text { Legumes } \\
\text { Relative Amt - } \\
\text { Post Legume } \\
\text { Relative }\end{array}$ & -0.19 & 0.66 & 0.18 & -0.59 & 0.20 & -1.04 & 12 \\
\hline $\begin{array}{l}\text { Pair } \\
18\end{array}$ & $\begin{array}{l}\text { Base Total } \\
\text { Relative Amt - } \\
\text { Post Total } \\
\text { Relative Amt }\end{array}$ & -0.58 & 1.40 & 0.39 & -1.43 & 0.26 & -1.5 & 12 \\
\hline $\begin{array}{l}\text { Pair } \\
19\end{array}$ & $\begin{array}{l}\text { Survey } 2 \text { Score } \\
- \\
\text { Post Survey } 2 \\
\text { Score }\end{array}$ & 0.58 & 1.88 & 0.54 & -0.61 & 1.77 & 1.07 & 11 \\
\hline
\end{tabular}




\begin{tabular}{|c|c|c|c|c|c|}
\hline \multicolumn{6}{|c|}{ I-3 Intervention Group: Correlations } \\
\hline & & $\begin{array}{l}\text { Base } \\
\text { Both } \\
\text { Obs }\end{array}$ & $\begin{array}{l}\text { Base Total } \\
\text { Consumption } \\
\text { Obs }\end{array}$ & $\begin{array}{l}\text { Post Both } \\
\text { Obs }\end{array}$ & $\begin{array}{c}\text { Post Total } \\
\text { Consumption } \\
\text { Obs }\end{array}$ \\
\hline \multirow{3}{*}{$\begin{array}{l}\text { Base Both } \\
\text { Obs }\end{array}$} & $\begin{array}{l}\text { Pearson } \\
\text { Correlation }\end{array}$ & 1.00 & 0.37 & $.754^{* *}$ & $.807^{* *}$ \\
\hline & Sig. (2-tailed) & & 0.16 & 0.00 & 0.00 \\
\hline & $\mathrm{N}$ & 16.00 & 16.00 & 16.00 & 16.00 \\
\hline \multirow{3}{*}{$\begin{array}{l}\text { Base Total } \\
\text { Consumption } \\
\text { Obs }\end{array}$} & $\begin{array}{l}\text { Pearson } \\
\text { Correlation }\end{array}$ & 0.37 & 1.00 & 0.26 & 0.30 \\
\hline & Sig. (2-tailed) & 0.16 & & 0.34 & 0.26 \\
\hline & $\mathrm{N}$ & 16.00 & 16.00 & 16.00 & 16.00 \\
\hline \multirow{3}{*}{ Post Both Obs } & $\begin{array}{l}\text { Pearson } \\
\text { Correlation }\end{array}$ & $.754^{* *}$ & 0.26 & 1.00 & $.654^{* *}$ \\
\hline & Sig. (2-tailed) & 0.00 & 0.34 & & 0.01 \\
\hline & $\mathrm{N}$ & 16.00 & 16.00 & 16.00 & 16.00 \\
\hline \multirow{3}{*}{$\begin{array}{l}\text { Post Total } \\
\text { Consumption } \\
\text { Obs }\end{array}$} & $\begin{array}{l}\text { Pearson } \\
\text { Correlation }\end{array}$ & $.807^{* *}$ & 0.30 & $.654^{* *}$ & 1.00 \\
\hline & Sig. (2-tailed) & 0.00 & 0.26 & 0.01 & \\
\hline & $\mathrm{N}$ & 16.00 & 16.00 & 16.00 & 16.00 \\
\hline
\end{tabular}




\begin{tabular}{|c|c|c|c|c|c|}
\hline \multicolumn{6}{|c|}{ I-3 Control Group: Correlations } \\
\hline & & $\begin{array}{l}\text { Base } \\
\text { Both } \\
\text { obs }\end{array}$ & $\begin{array}{l}\text { Base Total } \\
\text { Consumption } \\
\text { obs }\end{array}$ & $\begin{array}{c}\text { Post } \\
\text { Both } \\
\text { obs }\end{array}$ & $\begin{array}{l}\text { Post Total } \\
\text { Consumption } \\
\text { obs }\end{array}$ \\
\hline \multirow{3}{*}{ Base Both } & $\begin{array}{l}\text { Pearson } \\
\text { Correlation }\end{array}$ & 1.00 & 0.30 & $.845^{* *}$ & 0.43 \\
\hline & $\begin{array}{l}\text { Sig. (2- } \\
\text { tailed) }\end{array}$ & & 0.32 & 0.00 & 0.14 \\
\hline & $\mathrm{N}$ & $\begin{array}{r}13.0 \\
0\end{array}$ & 13.00 & 13.00 & 13.00 \\
\hline \multirow{3}{*}{$\begin{array}{l}\text { Base Total } \\
\text { Consumption obs }\end{array}$} & $\begin{array}{l}\text { Pearson } \\
\text { Correlation }\end{array}$ & 0.30 & 1.00 & $.600^{*}$ & 0.41 \\
\hline & $\begin{array}{l}\text { Sig. (2- } \\
\text { tailed) }\end{array}$ & 0.32 & & 0.03 & 0.16 \\
\hline & $\mathrm{N}$ & $\begin{array}{r}13.0 \\
0\end{array}$ & 13.00 & 13.00 & 13.00 \\
\hline \multirow{3}{*}{ Post Both } & $\begin{array}{l}\text { Pearson } \\
\text { Correlation }\end{array}$ & $.845^{*}$ & $.600^{*}$ & 1.00 & $.657^{*}$ \\
\hline & $\begin{array}{l}\text { Sig. (2- } \\
\text { tailed) }\end{array}$ & 0.00 & 0.03 & & 0.02 \\
\hline & $\mathrm{N}$ & $\begin{array}{r}13.0 \\
0\end{array}$ & 13.00 & 13.00 & 13.00 \\
\hline \multirow{3}{*}{$\begin{array}{l}\text { Post Total } \\
\text { Consumption obs }\end{array}$} & $\begin{array}{l}\text { Pearson } \\
\text { Correlation }\end{array}$ & 0.43 & 0.41 & $.657^{*}$ & 1.00 \\
\hline & $\begin{array}{l}\text { Sig. (2- } \\
\text { tailed) }\end{array}$ & 0.14 & 0.16 & 0.02 & \\
\hline & $\mathrm{N}$ & $\begin{array}{r}13.0 \\
0\end{array}$ & 13.00 & 13.00 & 13.00 \\
\hline
\end{tabular}

**. Correlation is significant at the 0.01 level (2-tailed).

*. Correlation is significant at the 0.05 level (2-tailed). 


\begin{tabular}{|c|c|c|c|c|c|}
\hline \multicolumn{5}{|c|}{ I-3 Both Groups PEARSON CORRELATION } & \multirow{2}{*}{$\begin{array}{c}\text { Two } \\
\text { tailed } \\
\text { sig. (p) }\end{array}$} \\
\hline & Mean & $\begin{array}{c}\text { Std. } \\
\text { Deviation }\end{array}$ & $\mathbf{N}$ & $\begin{array}{c}\text { Pearson } \\
\text { Correlation }\end{array}$ & \\
\hline BASE F/V OBSERVATION & 1.30 & 0.77 & 29 & $r=0.35$ & $p=0.06$ \\
\hline $\begin{array}{l}\text { BASELINE TOTAL } \\
\text { CONSUMPTION }\end{array}$ & 1.14 & 0.58 & 29 & & \\
\hline $\begin{array}{l}\text { POST STUDY F/V } \\
\text { OBSERVATION }\end{array}$ & 1.22 & 0.87 & 29 & $r=0.66$ & $p=0.00$ \\
\hline $\begin{array}{l}\text { POST STUDY TOTAL } \\
\text { CONSUMPTION }\end{array}$ & 0.95 & 0.81 & 29 & & \\
\hline
\end{tabular}


Table I-4: ANOVA Comparing Personal Data Measures

\begin{tabular}{|c|c|c|c|c|c|c|}
\hline \multicolumn{7}{|c|}{$\begin{array}{l}\text { I-4 ANOVA COMPARING PRE/POST HEIGHT, WEIGHT, BMI, \& } \\
\text { BLOOD PRESSURE }\end{array}$} \\
\hline & & $\begin{array}{l}\text { Sum of } \\
\text { Squares }\end{array}$ & $\mathrm{df}$ & $\begin{array}{l}\text { Mean } \\
\text { Square }\end{array}$ & $\mathrm{F}$ & Sig. \\
\hline \multirow{3}{*}{ Height } & $\begin{array}{l}\text { Between } \\
\text { Groups }\end{array}$ & 7.76 & 1.00 & 7.76 & 0.16 & 0.70 \\
\hline & $\begin{array}{l}\text { Within } \\
\text { Groups }\end{array}$ & 1349.16 & 27.00 & 49.97 & & \\
\hline & Total & 1356.91 & 28.00 & & & \\
\hline \multirow{3}{*}{ Post height } & $\begin{array}{l}\text { Between } \\
\text { Groups }\end{array}$ & 20.61 & 1.00 & 20.61 & 0.38 & 0.54 \\
\hline & $\begin{array}{l}\text { Within } \\
\text { Groups }\end{array}$ & 1473.17 & 27.00 & 54.56 & & \\
\hline & Total & 1493.78 & 28.00 & & & \\
\hline \multirow{3}{*}{ Weight } & $\begin{array}{l}\text { Between } \\
\text { Groups }\end{array}$ & 3.06 & 1.00 & 3.06 & 0.03 & 0.86 \\
\hline & $\begin{array}{l}\text { Within } \\
\text { Groups }\end{array}$ & 2455.41 & 27.00 & 90.94 & & \\
\hline & Total & 2458.47 & 28.00 & & & \\
\hline \multirow{3}{*}{ Post weight } & $\begin{array}{l}\text { Between } \\
\text { Groups }\end{array}$ & 3.63 & 1.00 & 3.63 & 0.04 & 0.85 \\
\hline & $\begin{array}{l}\text { Within } \\
\text { Groups }\end{array}$ & 2537.33 & 27.00 & 93.98 & & \\
\hline & Total & 2540.96 & 28.00 & & & \\
\hline \multirow{3}{*}{ BMI } & $\begin{array}{l}\text { Between } \\
\text { Groups }\end{array}$ & 0.17 & 1.00 & 0.17 & 0.02 & 0.90 \\
\hline & $\begin{array}{l}\text { Within } \\
\text { Groups }\end{array}$ & 285.51 & 27.00 & 10.58 & & \\
\hline & Total & 285.68 & 28.00 & & & \\
\hline \multirow{3}{*}{ Post BMI } & $\begin{array}{l}\text { Between } \\
\text { Groups }\end{array}$ & 0.67 & 1.00 & 0.67 & 0.07 & 0.80 \\
\hline & $\begin{array}{l}\text { Within } \\
\text { Groups }\end{array}$ & 268.09 & 27.00 & 9.93 & & \\
\hline & Total & 268.76 & 28.00 & & & \\
\hline Pre systolic & $\begin{array}{l}\text { Between } \\
\text { Groups }\end{array}$ & 124.91 & 1.00 & 124.91 & 1.47 & 0.24 \\
\hline
\end{tabular}


Table I-5: School Lunch Observations for Intervention and Control Groups

\begin{tabular}{|c|c|c|c|c|c|c|c|c|}
\hline \multicolumn{9}{|c|}{ I-5 LUNCH MEAL OBSERVATIONS DESCRIPTIVES } \\
\hline & & \multirow{2}{*}{$\mathrm{N}$} & \multirow{2}{*}{ Mean } & \multirow{2}{*}{$\mathrm{SD}^{*}$} & \multirow{2}{*}{$\begin{array}{l}\text { Std. } \\
\text { Error }\end{array}$} & \multicolumn{2}{|c|}{$\begin{array}{c}95 \% \text { Confidence } \\
\text { Interval for } \\
\text { Mean } \\
\end{array}$} & \multirow{2}{*}{$\begin{array}{l}\text { Mini } \\
\text { mum }\end{array}$} \\
\hline & & & & & & $\begin{array}{l}\text { Lower } \\
\text { Bound }\end{array}$ & $\begin{array}{l}\text { Upper } \\
\text { Bound }\end{array}$ & \\
\hline \multirow{3}{*}{$\begin{array}{l}\text { Base Fruit } \\
\text { Obs }\end{array}$} & Intervention & 16.00 & 1.01 & 0.54 & 0.14 & 0.72 & 1.29 & 0.00 \\
\hline & Control & 13.00 & 1.11 & 0.46 & 0.13 & 0.84 & 1.39 & 0.50 \\
\hline & Total & 29.00 & 1.05 & 0.50 & 0.09 & 0.86 & 1.24 & 0.00 \\
\hline \multirow{3}{*}{ Base Veg Obs } & Intervention & 16.00 & 0.33 & 0.60 & 0.15 & 0.01 & 0.65 & 0.00 \\
\hline & Control & 13.00 & 0.21 & 0.31 & 0.08 & 0.03 & 0.40 & 0.00 \\
\hline & Total & 29.00 & 0.28 & 0.49 & 0.09 & 0.09 & 0.46 & 0.00 \\
\hline \multirow{3}{*}{ Base F/V Obs } & Intervention & 16.00 & 1.34 & 0.97 & 0.24 & 0.82 & 1.85 & 0.00 \\
\hline & Control & 13.00 & 1.26 & 0.47 & 0.13 & 0.98 & 1.54 & 0.67 \\
\hline & Total & 29.00 & 1.30 & 0.77 & 0.14 & 1.01 & 1.60 & 0.00 \\
\hline \multirow{3}{*}{$\begin{array}{l}\text { Base Total } \\
\text { Consumption } \\
\text { Obs }\end{array}$} & Intervention & 16.00 & 1.14 & 0.64 & 0.16 & 0.80 & 1.48 & 0.00 \\
\hline & Control & 13.00 & 1.14 & 0.51 & 0.14 & 0.83 & 1.45 & 0.33 \\
\hline & Total & 29.00 & 1.14 & 0.58 & 0.11 & 0.92 & 1.36 & 0.00 \\
\hline \multirow{3}{*}{ Post Fruit Obs } & Intervention & 16.00 & 1.30 & 0.93 & 0.23 & 0.80 & 1.79 & 0.00 \\
\hline & Control & 13.00 & 1.02 & 0.65 & 0.18 & 0.63 & 1.41 & 0.00 \\
\hline & Total & 29.00 & 1.17 & 0.81 & 0.15 & 0.86 & 1.48 & 0.00 \\
\hline \multirow{3}{*}{ Post Veg Obs } & Intervention & 16.00 & 0.03 & 0.13 & 0.03 & -0.04 & 0.10 & 0.00 \\
\hline & Control & 13.00 & 0.08 & 0.28 & 0.08 & $\begin{array}{l}-0.09 \\
\end{array}$ & 0.24 & 0.00 \\
\hline & Total & 29.00 & 0.05 & 0.20 & 0.04 & -0.03 & 0.13 & 0.00 \\
\hline \multirow{3}{*}{ Post Both Obs } & Intervention & 16.00 & 1.33 & 0.97 & 0.24 & 0.81 & 1.84 & 0.00 \\
\hline & Control & 13.00 & 1.10 & 0.76 & 0.21 & 0.64 & 1.56 & 0.00 \\
\hline & Total & 29.00 & 1.22 & 0.87 & 0.16 & 0.89 & 1.56 & 0.00 \\
\hline \multirow{3}{*}{$\begin{array}{l}\text { Post Total } \\
\text { Consumption }\end{array}$} & Intervention & 16.00 & 1.05 & 0.83 & 0.21 & 0.61 & 1.50 & 0.00 \\
\hline & Control & 13.00 & 0.83 & 0.79 & 0.22 & 0.35 & 1.30 & 0.00 \\
\hline & Total & 29.00 & 0.95 & 0.81 & 0.15 & 0.65 & 1.26 & 0.00 \\
\hline
\end{tabular}

*SD=standard deviation 


\begin{tabular}{|l|r|r|r|r|}
\hline \multicolumn{5}{|c|}{ I-5 TEST OF HOMOGENEITY OF VARIANCES } \\
\hline & $\begin{array}{c}\text { Levene } \\
\text { Statistic }\end{array}$ & df1 & \multicolumn{1}{c|}{ df2 } & \multicolumn{1}{|c|}{ Sig. } \\
\hline Base Fruit Obs & 0.46 & 1.00 & 27.00 & 0.50 \\
\hline Base Veg Obs & 1.14 & 1.00 & 27.00 & 0.30 \\
\hline Base Both Obs & 1.12 & 1.00 & 27.00 & 0.30 \\
\hline $\begin{array}{l}\text { Base Total } \\
\text { Consumption Obs }\end{array}$ & 0.07 & 1.00 & 27.00 & 0.79 \\
\hline Post Fruit Obs & 2.19 & 1.00 & 27.00 & 0.15 \\
\hline Post Veg Obs & 1.60 & 1.00 & 27.00 & 0.22 \\
\hline Post Both Obs & 1.02 & 1.00 & 27.00 & 0.32 \\
\hline $\begin{array}{l}\text { Post Total } \\
\text { Consumption Obs }\end{array}$ & 0.00 & 1.00 & 27.00 & 0.98 \\
\hline
\end{tabular}


I-5 ANOVA

\begin{tabular}{|c|c|c|c|c|c|c|}
\hline & & $\begin{array}{l}\text { Sum of } \\
\text { Squares }\end{array}$ & $\mathrm{df}$ & $\begin{array}{c}\text { Mean } \\
\text { Square }\end{array}$ & $\mathrm{F}$ & Sig. \\
\hline \multirow{3}{*}{ Base Fruit Obs } & $\begin{array}{l}\text { Between } \\
\text { Groups }\end{array}$ & 0.08 & 1.00 & 0.08 & 0.33 & 0.57 \\
\hline & Within Groups & 6.92 & 27.00 & 0.26 & & \\
\hline & Total & 7.01 & 28.00 & & & \\
\hline \multirow{3}{*}{ Base Veg Obs } & $\begin{array}{l}\text { Between } \\
\text { Groups }\end{array}$ & 0.11 & 1.00 & 0.11 & 0.44 & 0.51 \\
\hline & Within Groups & 6.51 & 27.00 & 0.24 & & \\
\hline & Total & 6.62 & 28.00 & & & \\
\hline \multirow{3}{*}{ Base Both Obs } & $\begin{array}{l}\text { Between } \\
\text { Groups }\end{array}$ & 0.04 & 1.00 & 0.04 & 0.07 & 0.80 \\
\hline & Within Groups & 16.66 & 27.00 & 0.62 & & \\
\hline & Total & 16.71 & 28.00 & & & \\
\hline \multirow{3}{*}{$\begin{array}{l}\text { Base Total Consumption } \\
\text { Obs }\end{array}$} & $\begin{array}{l}\text { Between } \\
\text { Groups }\end{array}$ & 0.00 & 1.00 & 0.00 & 0.00 & 0.99 \\
\hline & Within Groups & 9.31 & 27.00 & 0.35 & & \\
\hline & Total & 9.31 & 28.00 & & & \\
\hline \multirow{3}{*}{ Post Fruit Obs } & $\begin{array}{l}\text { Between } \\
\text { Groups }\end{array}$ & 0.55 & 1.00 & 0.55 & 0.83 & 0.37 \\
\hline & Within Groups & 17.96 & 27.00 & 0.67 & & \\
\hline & Total & 18.51 & 28.00 & & & \\
\hline \multirow{3}{*}{ Post Veg Obs } & $\begin{array}{l}\text { Between } \\
\text { Groups }\end{array}$ & 0.02 & 1.00 & 0.02 & 0.35 & 0.56 \\
\hline & Within Groups & 1.16 & 27.00 & 0.04 & & \\
\hline & Total & 1.17 & 28.00 & & & \\
\hline \multirow{3}{*}{ Post Both Obs } & $\begin{array}{l}\text { Between } \\
\text { Groups }\end{array}$ & 0.39 & 1.00 & 0.39 & 0.50 & 0.49 \\
\hline & Within Groups & 21.03 & 27.00 & 0.78 & & \\
\hline & Total & 21.42 & 28.00 & & & \\
\hline \multirow{3}{*}{$\begin{array}{l}\text { Post Total Consumption } \\
\text { Obs }\end{array}$} & $\begin{array}{l}\text { Between } \\
\text { Groups }\end{array}$ & 0.37 & 1.00 & 0.37 & 0.56 & 0.46 \\
\hline & Within Groups & 17.79 & 27.00 & 0.66 & & \\
\hline & Total & 18.16 & 28.00 & & & \\
\hline
\end{tabular}


Table I-6: ANOVA for the "Farm to Table" (Survey 1) for attitude, knowledge, and self-efficacy/perception

\begin{tabular}{|c|c|c|c|c|c|c|}
\hline \multicolumn{7}{|c|}{ I-6 ANOVA } \\
\hline & & $\begin{array}{l}\text { Sum of } \\
\text { Squares }\end{array}$ & df & $\begin{array}{l}\text { Mean } \\
\text { Square }\end{array}$ & $\mathrm{F}$ & Sig. \\
\hline \multirow{3}{*}{ Base Attitude } & $\begin{array}{l}\text { Between } \\
\text { Groups }\end{array}$ & 280.96 & 1.00 & 280.96 & 3.13 & 0.09 \\
\hline & $\begin{array}{l}\text { Within } \\
\text { Groups }\end{array}$ & 2242.01 & 25.00 & 89.68 & & \\
\hline & Total & 2522.96 & 26.00 & & & \\
\hline \multirow{3}{*}{ Post Attitude } & $\begin{array}{l}\text { Between } \\
\text { Groups }\end{array}$ & 125.77 & 1.00 & 125.77 & 1.60 & 0.22 \\
\hline & $\begin{array}{l}\text { Within } \\
\text { Groups }\end{array}$ & 2120.44 & 27.00 & 78.54 & & \\
\hline & Total & 2246.21 & 28.00 & & & \\
\hline \multirow{3}{*}{ Base Knowledge } & $\begin{array}{l}\text { Between } \\
\text { Groups }\end{array}$ & 2.55 & 1.00 & 2.55 & 1.25 & 0.27 \\
\hline & $\begin{array}{l}\text { Within } \\
\text { Groups }\end{array}$ & 51.08 & 25.00 & 2.04 & & \\
\hline & Total & 53.63 & 26.00 & & & \\
\hline \multirow{3}{*}{ Post Knowledge } & $\begin{array}{l}\text { Between } \\
\text { Groups }\end{array}$ & 31.80 & 1.00 & 31.80 & 13.00 & 0.00 \\
\hline & $\begin{array}{l}\text { Within } \\
\text { Groups }\end{array}$ & 66.06 & 27.00 & 2.45 & & \\
\hline & Total & 97.86 & 28.00 & & & \\
\hline \multirow{3}{*}{ Base Perception/Self-Efficacy } & $\begin{array}{l}\text { Between } \\
\text { Groups }\end{array}$ & 2.60 & 1.00 & 2.60 & 0.28 & 0.60 \\
\hline & $\begin{array}{l}\text { Within } \\
\text { Groups }\end{array}$ & 229.70 & 25.00 & 9.19 & & \\
\hline & Total & 232.30 & 26.00 & & & \\
\hline \multirow{3}{*}{ Post Perception/Self-Efficacy } & $\begin{array}{l}\text { Between } \\
\text { Groups }\end{array}$ & 0.96 & 1.00 & 0.96 & 0.12 & 0.74 \\
\hline & $\begin{array}{l}\text { Within } \\
\text { Groups }\end{array}$ & 222.08 & 27.00 & 8.23 & & \\
\hline & Total & 223.03 & 28.00 & & & \\
\hline
\end{tabular}


Table I-7: ANOVA and descriptive statistics for the "Day in the Life Questionnaire" (DILQ-Survey 2) post study for all day dietary recall reported by the students.

\begin{tabular}{|c|c|c|c|c|c|c|}
\hline \multicolumn{7}{|c|}{ I-7 DESCRIPTIVES } \\
\hline \multicolumn{7}{|c|}{ POST STUDY DILQ ALL DAY DIETARY RECALL } \\
\hline & \multirow{2}{*}{$\mathrm{N}$} & \multirow{2}{*}{ Mean } & \multirow{2}{*}{$\begin{array}{c}\text { Std. } \\
\text { Deviation }\end{array}$} & \multirow{2}{*}{ Std. Error } & \multicolumn{2}{|c|}{$\begin{array}{l}95 \% \text { Confidence } \\
\text { Interval for Mean }\end{array}$} \\
\hline & & & & & $\begin{array}{l}\text { Lower } \\
\text { Bound }\end{array}$ & $\begin{array}{l}\text { Upper } \\
\text { Bound }\end{array}$ \\
\hline Intervention & 9 & 2.11 & 1.69 & 0.56 & 0.81 & 3.41 \\
\hline Control & 12 & 2.75 & 1.06 & 0.30 & 2.08 & 3.42 \\
\hline Total & 21 & 2.48 & 1.36 & 0.30 & 1.86 & 3.10 \\
\hline
\end{tabular}

\begin{tabular}{|l|r|r|r|r|c|}
\hline \multicolumn{7}{|c|}{ I-7 ANOVA } \\
\hline \multicolumn{7}{|c|}{ POST STUDY DILQ ALL DAY DIETARY RECALL } \\
\hline & $\begin{array}{r}\text { Sum of } \\
\text { Squares }\end{array}$ & df & $\begin{array}{c}\text { Mean } \\
\text { Square }\end{array}$ & F & Sig. \\
\hline $\begin{array}{l}\text { Between } \\
\text { Groups }\end{array}$ & 2.099 & 1 & 2.099 & 1.135 & 0.3 \\
\hline $\begin{array}{l}\text { Within } \\
\text { Groups }\end{array}$ & 35.139 & 19 & 1.849 & & \\
\hline Total & 37.238 & 20 & & & \\
\hline
\end{tabular}

\begin{tabular}{|c|c|c|c|c|c|}
\hline \multicolumn{7}{|c|}{ I-7 GROUP STATISTICS } \\
\hline & GROUP & $\mathrm{N}$ & Mean & Std. Deviation & Std. Error Mean \\
\hline \multirow{2}{*}{ Survey 2 score } & Intervention & 14 & 2.29 & 1.68 & 0.45 \\
\cline { 2 - 6 } & Control & 13 & 2.31 & 1.44 & 0.40 \\
\hline \multirow{2}{*}{ Post survey 2score } & Intervention & 9 & 1.44 & 1.42 & 0.47 \\
\cline { 2 - 6 } & Control & 12 & 1.58 & 1.16 & 0.34 \\
\hline
\end{tabular}


Table I-8: Mean and standard deviations for the adapted Eating at America's Table (EATS-Survey 3) parent report of fruit and vegetables (F/V) offered to and consumed by their child.

\begin{tabular}{|c|c|c|c|c|c|c|c|c|c|c|}
\hline \multicolumn{11}{|c|}{ I-8 Means and Standard Deviations } \\
\hline \multicolumn{2}{|c|}{ GROUP } & $\begin{array}{l}\text { Juice } \\
\text { Avg }\end{array}$ & $\begin{array}{l}\text { Fruit } \\
\text { Unit }\end{array}$ & $\begin{array}{l}\text { Fruit } \\
\text { Cups }\end{array}$ & $\begin{array}{l}\text { Lettuce } \\
\text { Cups }\end{array}$ & $\begin{array}{l}\text { French } \\
\text { Fries }\end{array}$ & $\begin{array}{l}\text { Other } \\
\text { Potatoes }\end{array}$ & $\begin{array}{l}\text { Dried } \\
\text { Beans }\end{array}$ & $\begin{array}{l}\text { Other } \\
\text { Veg }\end{array}$ & $\begin{array}{l}\text { Tom. } \\
\text { Sauce }\end{array}$ \\
\hline \multirow{4}{*}{$\mathrm{I}^{*}$} & Mean & 0.44 & 1.36 & 0.16 & 0.04 & 0.03 & 0.06 & 0.73 & 0.12 & 0.02 \\
\hline & $\mathrm{N}$ & 14 & 14 & 14 & 14 & 14 & 14 & 14 & 14 & 14 \\
\hline & SD & 0.91 & 1.31 & 0.17 & 0.06 & 0.04 & 0.11 & 1.30 & 0.15 & 0.03 \\
\hline & SEM & 0.24 & 0.35 & 0.05 & 0.02 & 0.01 & 0.03 & 0.35 & 0.04 & 0.01 \\
\hline \multirow{4}{*}{$\mathrm{C}^{* *}$} & Mean & 0.34 & 1.67 & 0.46 & 0.08 & 0.02 & 0.07 & 0.52 & 0.23 & 0.04 \\
\hline & $\mathrm{N}$ & 10.00 & 10.00 & 10.00 & 10.00 & 10.00 & 10.00 & 10.00 & 10.00 & 10.00 \\
\hline & SD & 0.34 & 1.55 & 0.38 & 0.12 & 0.03 & 0.19 & 0.28 & 0.35 & 0.05 \\
\hline & SEM & 0.11 & 0.49 & 0.12 & 0.04 & 0.01 & 0.06 & 0.09 & 0.11 & 0.02 \\
\hline \multirow{4}{*}{$\begin{array}{l}\text { Tot } \\
\text { al }\end{array}$} & Mean & 0.40 & 1.49 & 0.28 & 0.06 & 0.03 & 0.07 & 0.64 & 0.17 & 0.03 \\
\hline & $\mathrm{N}$ & 24.00 & 24.00 & 24.00 & 24.00 & 24.00 & 24.00 & 24.00 & 24.00 & 24.00 \\
\hline & SD & 0.72 & 1.39 & 0.31 & 0.09 & 0.03 & 0.14 & 1.00 & 0.25 & 0.04 \\
\hline & SEM & 0.15 & 0.28 & 0.06 & 0.02 & 0.01 & 0.03 & 0.20 & 0.05 & 0.01 \\
\hline
\end{tabular}

*I=Intervention $* * \mathrm{C}=\mathrm{Control}$ 


\begin{tabular}{|c|c|c|c|c|c|c|}
\hline \multicolumn{7}{|c|}{ I-8 ANOVA Table } \\
\hline & & $\begin{array}{l}\text { Sum of } \\
\text { Squares }\end{array}$ & df & $\begin{array}{l}\text { Mean } \\
\text { Square }\end{array}$ & $\mathrm{F}$ & Sig. \\
\hline \multirow{3}{*}{ Juice avg * Group } & Between Groups & 0.06 & 1 & 0.06 & 0.11 & 0.75 \\
\hline & Within Groups & 11.88 & 22 & 0.54 & & \\
\hline & Total & 11.93 & 23 & & & \\
\hline \multirow{3}{*}{ Fruit units * Group } & Between Groups & 0.58 & 1 & 0.58 & 0.29 & 0.60 \\
\hline & Within Groups & 43.75 & 22 & 1.99 & & \\
\hline & Total & 44.33 & 23 & & & \\
\hline \multirow{3}{*}{ Fruit cups * Group } & Between Groups & 0.51 & 1 & 0.51 & 6.55 & 0.02 \\
\hline & Within Groups & 1.70 & 22 & 0.08 & & \\
\hline & Total & 2.21 & 23 & & & \\
\hline \multirow{3}{*}{ Lettuce cups * Group } & Between Groups & 0.01 & 1 & 0.01 & 0.84 & 0.37 \\
\hline & Within Groups & 0.17 & 22 & 0.01 & & \\
\hline & Total & 0.18 & 23 & & & \\
\hline \multirow{3}{*}{ French fries * Group } & Between Groups & 0.00 & 1 & 0.00 & 0.22 & 0.64 \\
\hline & Within Groups & 0.03 & 22 & 0.00 & & \\
\hline & Total & 0.03 & 23 & & & \\
\hline \multirow{3}{*}{ Other white potatoes * Group } & Between Groups & 0.00 & 1 & 0.00 & 0.04 & 0.84 \\
\hline & Within Groups & 0.47 & 22 & 0.02 & & \\
\hline & Total & 0.47 & 23 & & & \\
\hline \multirow{3}{*}{ Dried beans * Group } & Between Groups & 0.25 & 1 & 0.25 & 0.24 & 0.63 \\
\hline & Within Groups & 22.79 & 22 & 1.04 & & \\
\hline & Total & 23.04 & 23 & & & \\
\hline \multirow{3}{*}{ Other veg * Group } & Between Groups & 0.06 & 1 & 0.06 & 0.96 & 0.34 \\
\hline & Within Groups & 1.41 & 22 & 0.06 & & \\
\hline & Total & 1.48 & 23 & & & \\
\hline \multirow{3}{*}{ Tomato sauce * Group } & Between Groups & 0.00 & 1 & 0.00 & 1.14 & 0.30 \\
\hline & Within Groups & 0.04 & 22 & 0.00 & & \\
\hline & Total & 0.04 & 23 & & & \\
\hline \multirow{3}{*}{ Veg soup * Group } & Between Groups & 0.04 & 1 & 0.04 & 0.18 & 0.67 \\
\hline & Within Groups & 4.15 & 22 & 0.19 & & \\
\hline & Total & 4.18 & 23 & & & \\
\hline \multirow{3}{*}{ Total Eats * Group } & $\begin{array}{l}\text { Between } \\
\text { Groups }\end{array}$ & 2.17 & 1 & 2.17 & 0.24 & 0.63 \\
\hline & Within Groups & 181.30 & 20 & 9.07 & & \\
\hline & Total & 183.47 & 21 & & & \\
\hline
\end{tabular}


Table I-9: Mean, standard deviations, and ANOVA for the adapted YouthAdolescent Food Frequency Questionnaire (YAQ-Survey 4) for the weekly dietary recall.

\begin{tabular}{|l|r|r|r|r|r|r|}
\hline \multicolumn{7}{|c|}{ I-9 CASE PROCESSING SUMMARY } \\
\hline & \multicolumn{7}{|c|}{ Cases } \\
\cline { 2 - 7 } & \multicolumn{2}{|c|}{ Included } & \multicolumn{2}{|c|}{ Excluded } & \multicolumn{2}{|c|}{ Total } \\
\cline { 2 - 7 } & $\mathrm{N}$ & Percent & \multicolumn{1}{|c|}{$\mathrm{N}$} & Percent & \multicolumn{1}{c|}{$\mathrm{N}$} & Percent \\
\hline $\begin{array}{l}\text { Fruit Weekly(4) * } \\
\text { GROUP }\end{array}$ & 23 & $65.70 \%$ & 12 & $34.30 \%$ & 35 & $100 \%$ \\
\hline $\begin{array}{l}\text { Vegetable Weekly(4) } \\
\text { * GROUP }\end{array}$ & 23 & $65.70 \%$ & 12 & $34.30 \%$ & 35 & $100 \%$ \\
\hline $\begin{array}{l}\text { Potato Weekly(4) * } \\
\text { GROUP }\end{array}$ & 23 & $65.70 \%$ & 12 & $34.30 \%$ & 35 & $100 \%$ \\
\hline $\begin{array}{l}\text { Fiber/Grain Weekly(4) } \\
\text { * GROUP }\end{array}$ & 23 & $65.70 \%$ & 12 & $34.30 \%$ & 35 & $100 \%$ \\
\hline $\begin{array}{l}\text { Beans Weekly(4) * } \\
\text { GROUP }\end{array}$ & 23 & $65.70 \%$ & 12 & $34.30 \%$ & 35 & $100 \%$ \\
\hline $\begin{array}{l}\text { Total F/V Weekly(4) } \\
\text { * Group }\end{array}$ & 23 & $65.70 \%$ & 12 & $34.30 \%$ & 35 & $100 \%$ \\
\hline
\end{tabular}

\begin{tabular}{|c|c|c|c|c|c|c|}
\hline \multicolumn{7}{|c|}{$\begin{array}{l}\text { I-9 MEANS, STANDARD DEVIATIONS (SD), AND STANDARD ERROR OF } \\
\text { MEAN (SEM) }\end{array}$} \\
\hline \multicolumn{2}{|l|}{ GROUP } & $\begin{array}{c}\text { Fruit } \\
\text { Weekly(4) }\end{array}$ & $\begin{array}{l}\text { Vegetable } \\
\text { Weekly(4) }\end{array}$ & $\begin{array}{c}\text { Potato } \\
\text { Weekly(4) }\end{array}$ & $\begin{array}{c}\text { Fiber/Grain } \\
\text { Weekly(4) }\end{array}$ & $\begin{array}{c}\text { Beans } \\
\text { Weekly( } \\
\text { 4) }\end{array}$ \\
\hline \multirow{4}{*}{ Intervention } & Mean & 0.70 & 0.75 & 0.19 & 0.4 & 0.13 \\
\hline & $\mathrm{N}$ & 11.00 & 11 & 11 & 11 & 11 \\
\hline & SD & 0.21 & 0.32 & 0.15 & 0.27 & 0.16 \\
\hline & SEM & 0.06 & 0.1 & 0.04 & 0.08 & 0.05 \\
\hline \multirow{4}{*}{ Control } & Mean & 0.83 & 0.87 & 0.15 & 0.32 & 0.12 \\
\hline & $\mathrm{N}$ & 12.00 & 12 & 12 & 12 & 12 \\
\hline & SD & 0.27 & 0.27 & 0.14 & 0.37 & 0.13 \\
\hline & SEM & 0.08 & 0.08 & 0.04 & 0.11 & 0.04 \\
\hline \multirow{4}{*}{ Total } & Mean & 0.77 & 0.81 & 0.17 & 0.36 & 0.12 \\
\hline & $\mathrm{N}$ & 23.00 & 23 & 23 & 23 & 23 \\
\hline & SD & 0.25 & 0.29 & 0.14 & 0.32 & 0.14 \\
\hline & SEM & 0.05 & 0.06 & 0.03 & 0.07 & 0.03 \\
\hline
\end{tabular}




\begin{tabular}{|c|c|c|c|c|c|c|}
\hline \multicolumn{7}{|c|}{ I-9 ANOVA TABLE } \\
\hline & & $\begin{array}{c}\text { Sum of } \\
\text { Squares }\end{array}$ & df & $\begin{array}{l}\text { Mean } \\
\text { Square }\end{array}$ & $\mathrm{F}$ & Sig. \\
\hline \multirow{3}{*}{$\begin{array}{l}\text { Fruit } \\
\text { Weekly(4) * } \\
\text { GROUP }\end{array}$} & $\begin{array}{l}\text { Between } \\
\text { Groups }\end{array}$ & 0.1 & 1 & 0.1 & 1.7 & 0.206 \\
\hline & $\begin{array}{l}\text { Within } \\
\text { Groups }\end{array}$ & 1.236 & 21 & 0.059 & & \\
\hline & Total & 1.336 & 22 & & & \\
\hline \multirow{3}{*}{$\begin{array}{l}\text { Vegetable } \\
\text { Weekly(4) * } \\
\text { GROUP }\end{array}$} & $\begin{array}{l}\text { Between } \\
\text { Groups }\end{array}$ & 0.077 & 1 & 0.077 & 0.889 & 0.357 \\
\hline & $\begin{array}{l}\text { Within } \\
\text { Groups }\end{array}$ & 1.818 & 21 & 0.087 & & \\
\hline & Total & 1.895 & 22 & & & \\
\hline \multirow{3}{*}{$\begin{array}{l}\text { Potato } \\
\text { Weekly(4)* } \\
\text { GROUP }\end{array}$} & $\begin{array}{l}\text { Between } \\
\text { Groups }\end{array}$ & 0.009 & 1 & 0.009 & 0.441 & 0.514 \\
\hline & $\begin{array}{l}\text { Within } \\
\text { Groups }\end{array}$ & 0.438 & 21 & 0.021 & & \\
\hline & Total & 0.447 & 22 & & & \\
\hline \multirow{3}{*}{$\begin{array}{l}\text { Fiber/Grain } \\
\text { Weekly(4) * } \\
\text { GROUP }\end{array}$} & $\begin{array}{l}\text { Between } \\
\text { Groups }\end{array}$ & 0.038 & 1 & 0.038 & 0.354 & 0.558 \\
\hline & $\begin{array}{l}\text { Within } \\
\text { Groups }\end{array}$ & 2.243 & 21 & 0.107 & & \\
\hline & Total & 2.28 & 22 & & & \\
\hline \multirow{3}{*}{$\begin{array}{l}\text { Beans } \\
\text { Weekly(4)* } \\
\text { GROUP }\end{array}$} & \begin{tabular}{|l|} 
Between \\
Groups
\end{tabular} & 0.001 & 1 & 0.001 & 0.031 & 0.863 \\
\hline & \begin{tabular}{|l|} 
Within \\
Groups \\
\end{tabular} & 0.461 & 21 & 0.022 & & \\
\hline & Total & 0.461 & 22 & & & \\
\hline \multirow{3}{*}{$\begin{array}{l}\text { Total F/V } \\
\text { Weekly (4) * } \\
\text { Group }\end{array}$} & \begin{tabular}{|l|} 
Between \\
Groups \\
\end{tabular} & 0.223 & 1 & 0.223 & 0.854 & 0.366 \\
\hline & \begin{tabular}{|l|} 
Within \\
Groups \\
\end{tabular} & 5.477 & 21 & 0.261 & & \\
\hline & Total & 5.7 & 22 & & & \\
\hline
\end{tabular}


Table I-10: Mean, standard deviations, and ANOVA for the adapted YouthAdolescent Food Frequency Questionnaire (YAQ-Survey 4) for the monthly dietary recall.

\begin{tabular}{|c|c|c|c|c|c|c|}
\hline \multicolumn{7}{|c|}{ I-10 CASE PROCESSING SUMMARY } \\
\hline & \multicolumn{6}{|c|}{ Cases } \\
\hline & \multicolumn{2}{|c|}{ Included } & \multicolumn{2}{|c|}{ Excluded } & \multicolumn{2}{|c|}{ Total } \\
\hline & $\mathrm{N}$ & Percent & $\mathrm{N}$ & Percent & $\mathrm{N}$ & Percent \\
\hline $\begin{array}{l}\text { Grains Month * } \\
\text { Group }\end{array}$ & 23 & $65.70 \%$ & 12 & $34.30 \%$ & 35 & $100.00 \%$ \\
\hline $\begin{array}{l}\text { Potato Month * } \\
\text { Group }\end{array}$ & 23 & $65.70 \%$ & 12 & $34.30 \%$ & 35 & $100.00 \%$ \\
\hline $\begin{array}{l}\text { Fruit Month * } \\
\text { Group }\end{array}$ & 23 & $65.70 \%$ & 12 & $34.30 \%$ & 35 & $100.00 \%$ \\
\hline $\begin{array}{l}\text { Beans Month * } \\
\text { Group }\end{array}$ & 23 & $65.70 \%$ & 12 & $34.30 \%$ & 35 & $100.00 \%$ \\
\hline $\begin{array}{l}\text { Vegetable Month } \\
\text { * Group }\end{array}$ & 23 & $65.70 \%$ & 12 & $34.30 \%$ & 35 & $100.00 \%$ \\
\hline $\begin{array}{l}\text { Total F/V Month } \\
* \text { Group }\end{array}$ & 23 & $65.70 \%$ & 12 & $34.30 \%$ & 35 & $100.00 \%$ \\
\hline
\end{tabular}




\begin{tabular}{|c|c|c|c|c|c|c|c|}
\hline \multicolumn{8}{|c|}{$\begin{array}{l}\text { 1-10 MEANS, STANDARD DEVIATIONS, } \\
\text { STANDARD ERROR OF MEANS }\end{array}$} \\
\hline \multicolumn{2}{|l|}{ Group } & $\begin{array}{l}\text { Grains } \\
\text { Month }\end{array}$ & $\begin{array}{l}\text { Potato } \\
\text { Month }\end{array}$ & $\begin{array}{l}\text { Fruit } \\
\text { Month }\end{array}$ & $\begin{array}{l}\text { Beans } \\
\text { Month }\end{array}$ & $\begin{array}{l}\text { Vegetable } \\
\text { Month }\end{array}$ & $\begin{array}{l}\text { Total } \\
\text { Month }\end{array}$ \\
\hline \multirow{4}{*}{ Intervention } & Mean & 0.42 & 0.10 & 0.73 & 0.12 & 0.60 & 1.55 \\
\hline & $\mathrm{N}$ & 11 & 11 & 11 & 11 & 11 & 11 \\
\hline & SD & 0.12 & 0.04 & 0.23 & 0.07 & 0.29 & 0.51 \\
\hline & SEM & 0.04 & 0.01 & 0.07 & 0.02 & 0.09 & 0.15 \\
\hline \multirow{4}{*}{ Control } & Mean & 0.47 & 0.07 & 0.70 & 0.08 & 0.60 & 1.45 \\
\hline & $\mathrm{N}$ & 12.00 & 12.00 & 12.00 & 12.00 & 12.00 & 12.00 \\
\hline & $\mathrm{SD}$ & 0.24 & 0.04 & 0.26 & 0.07 & 0.20 & 0.43 \\
\hline & SEM & 0.07 & 0.01 & 0.08 & 0.02 & 0.06 & 0.13 \\
\hline \multirow{4}{*}{ Total } & Mean & 0.44 & 0.09 & 0.72 & 0.10 & 0.60 & 1.50 \\
\hline & $\mathrm{N}$ & 23.00 & 23.00 & 23.00 & 23.00 & 23.00 & 23.00 \\
\hline & $\mathrm{SD}$ & 0.19 & 0.04 & 0.24 & 0.07 & 0.24 & 0.46 \\
\hline & SEM & 0.04 & 0.01 & 0.05 & 0.02 & 0.05 & 0.10 \\
\hline
\end{tabular}




\begin{tabular}{|c|c|c|c|c|c|c|}
\hline \multicolumn{7}{|c|}{ 1-10 ANOVA TABLE } \\
\hline & & $\begin{array}{c}\text { Sum of } \\
\text { Squares }\end{array}$ & $\mathrm{df}$ & $\begin{array}{c}\text { Mean } \\
\text { Square }\end{array}$ & $\mathrm{F}$ & Sig. \\
\hline \multirow{3}{*}{ Grains Month * Group } & Between Groups & 0.02 & 1 & 0.02 & 0.45 & 0.51 \\
\hline & Within Groups & 0.80 & 21 & 0.04 & & \\
\hline & Total & 0.81 & 22 & & & \\
\hline \multirow{3}{*}{ Potato Month * Group } & Between Groups & 0.01 & 1 & 0.01 & 3.16 & 0.09 \\
\hline & Within Groups & 0.04 & 21 & 0.00 & & \\
\hline & Total & 0.04 & 22 & & & \\
\hline \multirow{3}{*}{ Fruit Month * Group } & Between Groups & 0.00 & 1 & 0.00 & 0.07 & 0.80 \\
\hline & Within Groups & 1.31 & 21 & 0.06 & & \\
\hline & Total & 1.32 & 22 & & & \\
\hline \multirow{3}{*}{ Beans Month * Group } & Between Groups & 0.01 & 1 & 0.01 & 2.18 & 0.16 \\
\hline & Within Groups & 0.11 & 21 & 0.01 & & \\
\hline & Total & 0.12 & 22 & & & \\
\hline \multirow{3}{*}{$\begin{array}{l}\text { Vegetable Month * } \\
\text { Group }\end{array}$} & Between Groups & 0.00 & 1 & 0.00 & 0.00 & 1.00 \\
\hline & Within Groups & 1.30 & 21 & 0.06 & & \\
\hline & Total & 1.30 & 22 & & & \\
\hline \multirow{3}{*}{ Total Month * Group } & Between Groups & 0.06 & 1 & 0.06 & 0.27 & 0.61 \\
\hline & Within Groups & 4.64 & 21 & 0.22 & & \\
\hline & Total & 4.69 & 22 & & & \\
\hline
\end{tabular}


Table I-11: Independent T-Test for Aim 2 baseline impact of gender, BMI, BMI Zscores, blood pressure on the presence of fruits and vegetables in packed school lunch.

\begin{tabular}{|l|l|r|r|r|r|}
\hline \multicolumn{7}{|c|}{ I-11 GROUP STATISTICS } \\
\hline \multirow{3}{*}{ Gender } & BaseBoth & N & Mean & $\begin{array}{c}\text { Std. } \\
\text { Deviation }\end{array}$ & $\begin{array}{c}\text { Std. } \\
\text { Error } \\
\text { Mean }\end{array}$ \\
\hline \multirow{2}{*}{ BMI } & Yes F/V in Lunch & 27 & 1.48 & 0.51 & 0.10 \\
\cline { 2 - 6 } & No F/V in Lunch & 2 & 1.50 & 0.71 & 0.50 \\
\hline \multirow{2}{*}{ Baseline Z Score } & Yes F/V in Lunch & 27 & 19.69 & 3.20 & 0.61 \\
\cline { 2 - 6 } & No F/V in Lunch & 2 & 16.43 & 0.64 & 0.46 \\
\hline \multirow{2}{*}{ Base Systolic } & Yes F/V in Lunch & 27 & 0.44 & 0.91 & 0.18 \\
\cline { 2 - 6 } & No F/V in Lunch & 2 & -0.75 & 0.35 & 0.25 \\
\hline \multirow{2}{*}{ Base Diastolic } & Yes F/V in Lunch & 27 & 102.41 & 9.45 & 1.82 \\
\cline { 2 - 6 } & No F/V in Lunch & 2 & 102.00 & 9.90 & 7.00 \\
\hline \multirow{2}{*}{ Base Both F/V Obs } & Yes F/V in Lunch & 27 & 63.30 & 7.21 & 1.39 \\
\cline { 2 - 6 } & No F/V in Lunch & 2 & 66.00 & 2.83 & 2.00 \\
\cline { 2 - 6 } & Yes F/V in Lunch & 27 & 1.34 & 0.70 & 0.14 \\
\cline { 2 - 6 } & No F/V in Lunch & 2 & 0.00 & 0.00 & 0.00 \\
\hline
\end{tabular}




\begin{tabular}{|c|c|c|c|c|c|c|c|c|c|c|}
\hline & \multicolumn{2}{|c|}{$\begin{array}{c}\text { Levene's } \\
\text { Test for } \\
\text { Equality of } \\
\text { Variances }\end{array}$} & \multicolumn{7}{|c|}{ t-test for Equality of Means } \\
\hline & & \multirow[t]{2}{*}{$\mathrm{F}$} & \multirow[t]{2}{*}{ Sig. } & \multirow[t]{2}{*}{$\mathrm{t}$} & \multirow[t]{2}{*}{ df } & \multirow{2}{*}{$\begin{array}{l}\text { Sig. } \\
2- \\
\text { tailed }\end{array}$} & \multirow[t]{2}{*}{$\begin{array}{c}\text { Mean } \\
\text { Difference }\end{array}$} & \multirow{2}{*}{$\begin{array}{l}\text { Std. } \\
\text { Error } \\
\text { Diff. }\end{array}$} & \multicolumn{2}{|c|}{$\begin{array}{l}95 \% \text { Confidence } \\
\text { Interval of the } \\
\text { Difference }\end{array}$} \\
\hline & & & & & & & & & Lower & Upper \\
\hline \multirow[b]{2}{*}{ Gender } & $\begin{array}{l}\text { Equal } \\
\text { variance } \\
\text { assumed }\end{array}$ & 0.00 & 0.96 & $\overline{-} .05$ & 27 & 0.96 & -0.02 & 0.38 & -0.80 & 0.76 \\
\hline & $\begin{array}{l}\text { Equal } \\
\text { variance } \\
\text { not } \\
\text { assumed }\end{array}$ & & & $\begin{array}{l}- \\
0.04\end{array}$ & 1.078 & 0.98 & -0.02 & 0.51 & -5.48 & 5.44 \\
\hline \multirow[b]{2}{*}{ BMI } & $\begin{array}{l}\text { Equal } \\
\text { variance } \\
\text { assumed }\end{array}$ & 2.53 & 0.12 & 1.42 & 27 & 0.17 & 3.26 & 2.30 & -1.46 & 7.98 \\
\hline & $\begin{array}{l}\text { Equal } \\
\text { variance } \\
\text { not } \\
\text { assumed }\end{array}$ & & & 4.26 & 7.063 & 0.00 & 3.26 & 0.77 & 1.45 & 5.07 \\
\hline \multirow{2}{*}{$\begin{array}{l}\text { Base } \\
\text { line Z } \\
\text { Score }\end{array}$} & $\begin{array}{l}\text { Equal } \\
\text { variance } \\
\text { assumed }\end{array}$ & 1.63 & 0.21 & 1.81 & 27 & 0.08 & 1.19 & 0.66 & -0.16 & 2.55 \\
\hline & $\begin{array}{l}\text { Equal } \\
\text { variance } \\
\text { not } \\
\text { assumed }\end{array}$ & & & 3.91 & 2.211 & 0.05 & 1.19 & 0.31 & -0.01 & 2.40 \\
\hline \multirow[b]{2}{*}{$\begin{array}{l}\text { Pre } \\
\text { systolic }\end{array}$} & $\begin{array}{l}\text { Equal } \\
\text { variance } \\
\text { assumed }\end{array}$ & 0.00 & 0.97 & 0.06 & 27 & 0.95 & 0.41 & 6.94 & -13.82 & 14.64 \\
\hline & $\begin{array}{l}\text { Equal } \\
\text { variance } \\
\text { not } \\
\text { assumed }\end{array}$ & & & 0.06 & 1.139 & 0.96 & 0.41 & 7.23 & -68.76 & 69.58 \\
\hline \multirow{2}{*}{$\begin{array}{l}\text { Pre } \\
\text { dias. }\end{array}$} & $\begin{array}{l}\text { Equal } \\
\text { variances } \\
\text { assumed }\end{array}$ & 1.44 & 0.24 & $\overline{-} .52$ & 27 & 0.61 & -2.70 & 5.20 & -13.37 & 7.97 \\
\hline & $\begin{array}{l}\text { Equal } \\
\text { variances } \\
\text { not } \\
\text { assumed }\end{array}$ & & & $\begin{array}{l}- \\
1.11\end{array}$ & 2.175 & 0.37 & -2.70 & 2.43 & -12.41 & 7.00 \\
\hline \multirow{2}{*}{$\begin{array}{l}\text { Base } \\
\text { Both } \\
\text { F/V } \\
\text { Obs }\end{array}$} & $\begin{array}{l}\text { Equal } \\
\text { variances } \\
\text { assumed }\end{array}$ & 1.59 & 0.22 & 2.65 & 27 & 0.01 & 1.34 & 0.51 & 0.30 & 2.37 \\
\hline & $\begin{array}{l}\text { Equal } \\
\text { variances } \\
\text { not } \\
\text { assumed }\end{array}$ & & & 9.89 & 26 & 0.00 & 1.34 & 0.14 & 1.06 & 1.61 \\
\hline
\end{tabular}


Table I-12: Independent T-Test for Aim 2 baseline impact of gender, BMI, BMI Zscores, blood pressure on the presence of fruits and vegetables in packed school lunch.

\begin{tabular}{|c|c|c|c|c|c|}
\hline \multicolumn{6}{|c|}{ 1-12 GROUP STATISTICS } \\
\hline & Post Both & $\mathrm{N}$ & Mean & $\begin{array}{c}\text { Std. } \\
\text { Deviation }\end{array}$ & $\begin{array}{c}\text { Std. } \\
\text { Error } \\
\text { Mean }\end{array}$ \\
\hline \multirow[b]{2}{*}{ Gender } & Yes F/V present in school lunch & 25 & 1.40 & 0.50 & 0.10 \\
\hline & $\begin{array}{l}\text { No F/V not present in school } \\
\text { lunch }\end{array}$ & 4 & 2.00 & 0.00 & 0.00 \\
\hline \multirow[b]{2}{*}{ Post BMI } & Yes F/V present in school lunch & 25 & 19.79 & 3.21 & 0.64 \\
\hline & $\begin{array}{l}\text { No F/V not present in school } \\
\text { lunch }\end{array}$ & 4 & 18.66 & 2.41 & 1.20 \\
\hline \multirow[b]{2}{*}{ Post Z Score } & Yes F/V present in school lunch & 25 & 0.54 & 0.87 & 0.17 \\
\hline & $\begin{array}{l}\text { No F/V not present in school } \\
\text { lunch }\end{array}$ & 4 & 0.75 & 0.65 & 0.32 \\
\hline \multirow[b]{2}{*}{ Post Systolic } & Yes F/V present in school lunch & 25 & 98.72 & 10.51 & 2.10 \\
\hline & $\begin{array}{l}\text { No F/V not present in school } \\
\text { lunch }\end{array}$ & 4 & 99.00 & 7.35 & 3.67 \\
\hline \multirow[b]{2}{*}{ Post Diastolic } & Yes F/V present in school lunch & 25 & 62.88 & 9.19 & 1.84 \\
\hline & $\begin{array}{l}\text { No F/V not present in school } \\
\text { lunch }\end{array}$ & 4 & 68.75 & 13.52 & 6.76 \\
\hline
\end{tabular}

\begin{tabular}{|c|c|c|}
\hline Group & $\begin{array}{c}\text { Post Study Presence of F/V } \\
\text { In School Lunch } \\
\text { Yes }\end{array}$ & $\begin{array}{c}\text { Post Study Presence of F/V } \\
\text { in School Lunch } \\
\text { No }\end{array}$ \\
\hline Female & 12 & 2 \\
\hline Male (number) & 12 & 3 \\
\hline
\end{tabular}




\begin{tabular}{|c|c|c|c|c|c|c|c|c|c|}
\hline & \multicolumn{2}{|c|}{$\begin{array}{c}\text { Levene's } \\
\text { Test for } \\
\text { Equality of } \\
\text { Variances }\end{array}$} & \multicolumn{6}{|c|}{ t-test for Equality of Means } \\
\hline & & \multirow[t]{2}{*}{$\mathrm{F}$} & \multirow[t]{2}{*}{ Sig. } & \multirow[t]{2}{*}{$\mathrm{t}$} & \multirow[t]{2}{*}{ df } & \multirow[t]{2}{*}{$\begin{array}{l}\text { Sig. } \\
2- \\
\text { tailed }\end{array}$} & \multirow[t]{2}{*}{$\begin{array}{l}\text { Mean } \\
\text { Difference }\end{array}$} & \multirow[t]{2}{*}{$\begin{array}{l}\text { Std. Error } \\
\text { Difference }\end{array}$} & $\begin{array}{l}95 \% \\
\text { Confidence } \\
\text { Interval of } \\
\text { the } \\
\text { Difference }\end{array}$ \\
\hline & & & & & & & & & Lower \\
\hline \multirow[b]{2}{*}{ Gender } & $\begin{array}{l}\text { Equal } \\
\text { variances } \\
\text { assumed }\end{array}$ & 89.38 & 0.00 & -2.36 & 27.00 & 0.03 & -0.60 & 0.25 & -1.12 \\
\hline & $\begin{array}{l}\text { Equal } \\
\text { variances } \\
\text { not } \\
\text { assumed }\end{array}$ & & & -6.00 & 24.00 & 0.00 & -0.60 & 0.10 & -0.81 \\
\hline \multirow[b]{2}{*}{$\begin{array}{l}\text { Post } \\
\text { BMI }\end{array}$} & $\begin{array}{l}\text { Equal } \\
\text { variances } \\
\text { assumed }\end{array}$ & 0.66 & 0.42 & 0.67 & 27.00 & 0.51 & 1.13 & 1.69 & -2.33 \\
\hline & $\begin{array}{l}\text { Equal } \\
\text { variances } \\
\text { not } \\
\text { assumed }\end{array}$ & & & 0.83 & 4.90 & 0.45 & 1.13 & 1.36 & -2.40 \\
\hline \multirow[b]{2}{*}{$\begin{array}{l}\text { Post Z } \\
\text { Score }\end{array}$} & $\begin{array}{l}\text { Equal } \\
\text { variances } \\
\text { assumed }\end{array}$ & 0.54 & 0.47 & -0.46 & 27.00 & 0.65 & -0.21 & 0.45 & -1.14 \\
\hline & $\begin{array}{l}\text { Equal } \\
\text { variances } \\
\text { not } \\
\text { assumed }\end{array}$ & & & -0.57 & 4.92 & 0.59 & -0.21 & 0.37 & -1.16 \\
\hline \multirow[b]{2}{*}{$\begin{array}{l}\text { Post } \\
\text { Systolic }\end{array}$} & $\begin{array}{l}\text { Equal } \\
\text { variances } \\
\text { assumed }\end{array}$ & 0.66 & 0.42 & -0.05 & 27.00 & 0.96 & -0.28 & 5.50 & -11.56 \\
\hline & $\begin{array}{l}\text { Equal } \\
\text { variances } \\
\text { not } \\
\text { assumed }\end{array}$ & & & -0.07 & 5.22 & 0.95 & -0.28 & 4.23 & -11.03 \\
\hline \multirow{2}{*}{$\begin{array}{l}\text { Post } \\
\text { Diastolic }\end{array}$} & $\begin{array}{l}\text { Equal } \\
\text { variances } \\
\text { assumed }\end{array}$ & 1.97 & 0.17 & -1.12 & 27.00 & 0.27 & -5.87 & 5.26 & -16.67 \\
\hline & $\begin{array}{l}\text { Equal } \\
\text { variances } \\
\text { not } \\
\text { assumed }\end{array}$ & & & -0.84 & 3.46 & 0.46 & -5.87 & 7.01 & -26.59 \\
\hline
\end{tabular}


Table I-13: Independent T-Test for parental involvement (grocery list completion) and post study observation and consumption for fruits and vegetables at school lunch

\begin{tabular}{|c|c|c|c|c|c|}
\hline \multicolumn{7}{|c|}{1 1-13 GROUP STATISTICS } \\
\hline & $\begin{array}{c}\text { PARENTAL } \\
\text { Involvement }\end{array}$ & $\mathrm{N}$ & Mean & Std. Deviation & $\begin{array}{c}\text { Std. } \\
\text { Error } \\
\text { Mean }\end{array}$ \\
\hline \multirow{2}{*}{ Post Both F/V } & yes & 10 & 1.48 & 0.90 & 0.28 \\
\cline { 2 - 6 } & no & 6 & 1.08 & 1.11 & 0.45 \\
\hline $\begin{array}{c}\text { Post Total } \\
\text { Consumption }\end{array}$ & yes & 10 & 1.48 & 0.90 & 0.28 \\
\cline { 2 - 6 } & no & 6 & 1.08 & 1.11 & 0.45 \\
\hline
\end{tabular}




\begin{tabular}{|c|c|c|c|c|c|c|c|c|c|}
\hline \multicolumn{10}{|c|}{ 1-13 INDEPENDENT SAMPLES TEST } \\
\hline & & \multicolumn{2}{|c|}{$\begin{array}{l}\text { Levene's } \\
\text { Test for } \\
\text { Equality } \\
\text { of } \\
\text { Variances }\end{array}$} & \multicolumn{6}{|c|}{ t-test for Equality of Means } \\
\hline & & $\mathrm{F}$ & Sig. & $\mathrm{t}$ & df & $\begin{array}{l}\text { Sig. } \\
(2- \\
\text { tailed) }\end{array}$ & $\begin{array}{c}\text { Mean } \\
\text { Difference }\end{array}$ & $\begin{array}{l}\text { Std. Error } \\
\text { Difference }\end{array}$ & $\begin{array}{c}95 \% \\
\text { Confidence } \\
\text { Interval of } \\
\text { the } \\
\text { Difference }\end{array}$ \\
\hline & & & & & & & & & Lower \\
\hline \multirow[b]{2}{*}{$\begin{array}{l}\text { Post Both } \\
\text { F/V }\end{array}$} & $\begin{array}{l}\text { Equal } \\
\text { variances } \\
\text { assumed }\end{array}$ & 0.10 & 0.75 & 0.77 & 14.00 & 0.45 & 0.39 & 0.51 & -0.70 \\
\hline & $\begin{array}{l}\text { Equal } \\
\text { variances } \\
\text { not } \\
\text { assumed }\end{array}$ & & & 0.73 & 8.93 & 0.48 & 0.39 & 0.54 & -0.82 \\
\hline \multirow[b]{2}{*}{$\begin{array}{l}\text { Post Total } \\
\text { Consumption }\end{array}$} & $\begin{array}{l}\text { Equal } \\
\text { variances } \\
\text { assumed }\end{array}$ & 0.10 & 0.75 & 0.77 & 14.00 & 0.45 & 0.39 & 0.51 & -0.70 \\
\hline & $\begin{array}{l}\text { Equal } \\
\text { variances } \\
\text { not } \\
\text { assumed }\end{array}$ & & & 0.73 & 8.93 & 0.48 & 0.39 & 0.54 & -0.82 \\
\hline
\end{tabular}


Table I-14: Correlation of observations and survey tools at baseline and post study.

\section{I-14 BASELINE}

\begin{tabular}{|l|r|r|r|}
\hline \multicolumn{4}{|c|}{ 1-14 DESCRIPTIVE STATISTICS } \\
\hline & \multicolumn{1}{|c|}{ Mean } & $\begin{array}{c}\text { Std. } \\
\text { Deviation }\end{array}$ & \multicolumn{1}{c|}{ N } \\
\hline Base Both F/V Obs & 1.30 & 0.77 & 29 \\
\hline Base Total Consumption & 1.14 & 0.58 & 29 \\
\hline Baseline Total Relative Amt & 1.72 & 1.06 & 29 \\
\hline Total Eats & 3.59 & 2.96 & 22 \\
\hline Total F/V Week(4) & 1.88 & 0.51 & 23 \\
\hline Total Month & 1.50 & 0.46 & 23 \\
\hline
\end{tabular}




\begin{tabular}{|c|c|c|c|c|c|c|c|}
\hline \multicolumn{8}{|c|}{ I-14 CORRELATIONS } \\
\hline & & $\begin{array}{l}\text { Base } \\
\text { Both } \\
\text { F/V }\end{array}$ & $\begin{array}{l}\text { Base Total } \\
\text { Consumption }\end{array}$ & $\begin{array}{c}\text { Base } \\
\text { Total } \\
\text { Relative } \\
\text { Amt }\end{array}$ & $\begin{array}{l}\text { Total } \\
\text { Eats }\end{array}$ & $\begin{array}{c}\text { Total } \\
\text { F/V } \\
\text { Weekly } \\
(4)\end{array}$ & $\begin{array}{c}\text { Total } \\
\text { Mont } \\
\mathrm{h}\end{array}$ \\
\hline \multirow{3}{*}{$\begin{array}{l}\text { Base Both } \\
\text { F/V }\end{array}$} & $\begin{array}{l}\text { Pearson } \\
\text { Correlation } \\
\end{array}$ & 1 & 0.348 & -0.051 & 0.11 & 0.315 & $.594^{*}$ \\
\hline & Sig. 2-tailed & & 0.064 & 0.793 & 0.625 & 0.143 & $\begin{array}{r}0.00 \\
3\end{array}$ \\
\hline & $\mathrm{N}$ & 29 & 29 & 29 & 22 & 23 & 23 \\
\hline \multirow{3}{*}{$\begin{array}{l}\text { Base Total } \\
\text { Consumption }\end{array}$} & $\begin{array}{l}\text { Pearson } \\
\text { Correlation }\end{array}$ & 0.348 & 1 & 0.215 & 0.053 & 0.325 & $\begin{array}{r}0.26 \\
8 \\
\end{array}$ \\
\hline & Sig. 2-tailed & 0.064 & & 0.263 & 0.815 & 0.131 & $\begin{array}{r}0.21 \\
7\end{array}$ \\
\hline & $\mathrm{N}$ & 29 & 29 & 29 & 22 & 23 & 23 \\
\hline \multirow{3}{*}{$\begin{array}{l}\text { Base Total } \\
\text { Relative Amt }\end{array}$} & $\begin{array}{l}\text { Pearson } \\
\text { Correlation }\end{array}$ & $\begin{array}{r}- \\
0.051\end{array}$ & 0.215 & 1 & 0.135 & 0.293 & $\begin{array}{r}0.20 \\
1\end{array}$ \\
\hline & Sig. 2-tailed & 0.793 & 0.263 & & 0.55 & 0.175 & $\begin{array}{r}0.35 \\
7\end{array}$ \\
\hline & $\mathrm{N}$ & 29 & 29 & 29 & 22 & 23 & 23 \\
\hline \multirow{3}{*}{ Total Eats } & $\begin{array}{l}\text { Pearson } \\
\text { Correlation } \\
\end{array}$ & 0.11 & -0.053 & 0.135 & 1 & 0.279 & $.503^{*}$ \\
\hline & Sig. 2-tailed & 0.625 & 0.815 & 0.55 & & 0.234 & $\begin{array}{r}0.02 \\
4 \\
\end{array}$ \\
\hline & $\mathrm{N}$ & 22 & 22 & 22 & 22 & 20 & 20 \\
\hline \multirow{3}{*}{$\begin{array}{l}\text { Total F/V } \\
\text { Weekly (4) }\end{array}$} & $\begin{array}{l}\text { Pearson } \\
\text { Correlation } \\
\end{array}$ & 0.315 & 0.325 & 0.293 & 0.279 & 1 & $\begin{array}{r}0.34 \\
6 \\
\end{array}$ \\
\hline & Sig. 2-tailed & 0.143 & 0.131 & 0.175 & 0.234 & & $\begin{array}{r}0.10 \\
6\end{array}$ \\
\hline & $\mathrm{N}$ & 23 & 23 & 23 & 20 & 23 & 23 \\
\hline \multirow{3}{*}{ Total Month } & $\begin{array}{l}\text { Pearson } \\
\text { Correlation }\end{array}$ & $.594^{* *}$ & 0.268 & 0.201 & $.503^{*}$ & 0.346 & 1 \\
\hline & Sig. 2-tailed & 0.003 & 0.217 & 0.357 & 0.024 & 0.106 & \\
\hline & $\mathrm{N}$ & 23 & 23 & 23 & 20 & 23 & 23 \\
\hline
\end{tabular}

**. Correlation is significant at the 0.01 level (2-tailed).

*. Correlation is significant at the 0.05 level (2-tailed). 


\section{I-14 Post Study Correlation of Surveys}

\begin{tabular}{|l|r|r|r|}
\hline \multicolumn{3}{|c|}{ I-14 DESCRIPTIVE STATISTICS } \\
\hline & \multicolumn{1}{|c|}{ Mean } & $\begin{array}{c}\text { Std. } \\
\text { Deviation }\end{array}$ & \multicolumn{1}{|c|}{ N } \\
\hline Post Both F/V & 1.22 & 0.87 & 29 \\
\hline Post Total Consumption & 1.10 & 0.91 & 29 \\
\hline Post Total Relative Amt & 2.48 & 1.49 & 29 \\
\hline Post Survey DILQ (2) Dinner & 2.48 & 1.36 & 21 \\
\hline
\end{tabular}




\begin{tabular}{|c|c|c|c|c|c|}
\hline \multicolumn{6}{|c|}{ 1-14 CORRELATIONS } \\
\hline & & $\begin{array}{l}\text { Post } \\
\text { Both } \\
\text { F/V }\end{array}$ & $\begin{array}{l}\text { Post Total } \\
\text { Consumption }\end{array}$ & $\begin{array}{c}\text { Post Total } \\
\text { Relative } \\
\text { Amt }\end{array}$ & $\begin{array}{c}\text { Post Survey DILQ (2) } \\
\text { Dinner }\end{array}$ \\
\hline \multirow{3}{*}{ Post Both F/V } & $\begin{array}{l}\text { Pearson } \\
\text { Correlation }\end{array}$ & 1.00 & $.879^{* *}$ & -0.22 & -0.14 \\
\hline & $\begin{array}{l}\text { Sig. (2- } \\
\text { tailed) }\end{array}$ & & 0.00 & 0.26 & 0.54 \\
\hline & $\mathrm{N}$ & 29.00 & 29.00 & 29.00 & 21.00 \\
\hline \multirow{3}{*}{$\begin{array}{l}\text { Post Total } \\
\text { Consumption }\end{array}$} & $\begin{array}{l}\text { Pearson } \\
\text { Correlation }\end{array}$ & $.879^{* *}$ & 1.00 & -0.08 & -0.10 \\
\hline & $\begin{array}{l}\text { Sig. }(2- \\
\text { tailed) }\end{array}$ & 0.00 & & 0.67 & 0.68 \\
\hline & $\mathrm{N}$ & 29.00 & 29.00 & 29.00 & 21.00 \\
\hline \multirow{3}{*}{$\begin{array}{l}\text { Post Total } \\
\text { Relative Amt }\end{array}$} & $\begin{array}{l}\text { Pearson } \\
\text { Correlation }\end{array}$ & -0.22 & -0.08 & 1.00 & 0.16 \\
\hline & $\begin{array}{l}\text { Sig. (2- } \\
\text { tailed) }\end{array}$ & 0.26 & 0.67 & & 0.49 \\
\hline & $\mathrm{N}$ & 29.00 & 29.00 & 29.00 & 21.00 \\
\hline \multirow{3}{*}{$\begin{array}{l}\text { Post Survey } \\
\text { DILQ (2) } \\
\text { Dinner }\end{array}$} & $\begin{array}{l}\text { Pearson } \\
\text { Correlation }\end{array}$ & -0.14 & -0.10 & 0.16 & 1.00 \\
\hline & $\begin{array}{l}\text { Sig. (2- } \\
\text { tailed) }\end{array}$ & 0.54 & 0.68 & 0.49 & \\
\hline & $\mathrm{N}$ & 21.00 & 21.00 & 21.00 & 21.00 \\
\hline
\end{tabular}

**. Correlation is significant at the 0.01 level (2-tailed). 


\section{Appendix J: Raw data for intervention and control group participants}

\begin{tabular}{|c|c|c|c|c|c|c|c|c|c|c|}
\hline Subj & Subj Sty & AGE & Gende & Height Pd & ht m & WT kg & Weight B & BMI & z score & $\mathrm{CDCPe}$ \\
\hline WB & 0026B & 12 & 1 & 168 & 1.68 & 56.3636 & 124 & 19.9701093 & & 150th-75 \\
\hline TB & $0036 \mathrm{~B}$ & 12 & 1 & 156 & 1.56 & 45.4545 & 100 & 18.6779033 & 0.5 & $5 \longdiv { 5 0 \text { th-75 } }$ \\
\hline WD & 0046B & 12 & 1 & 156 & 1.56 & 39.5455 & 87 & 16.2497759 & -0.5 & 10th-25 \\
\hline DF & 0076B & 12 & 1 & 159.5 & 1.59 & 41.3636 & 91 & 16.3615507 & -0.5 & 5 10th-25 \\
\hline AK & $0096 \mathrm{~B}$ & 12 & 2 & 155.5 & 1.55 & 57.3636 & 126.2 & 23.8766436 & 1.5 & 50th-95 \\
\hline JL & $00116 \mathrm{~B}$ & 12 & 1 & 151 & 1.51 & 46.3636 & 102 & 20.3340364 & 0.5 & 50 th-75 \\
\hline MP & $00146 \mathrm{~B}$ & 11 & 2 & 173.375 & 1.73 & 77.7273 & 171 & 25.9705546 & & $2>95$ th \\
\hline ER & $00166 \mathrm{~B}$ & 12 & 2 & 160 & 1.6 & 52.2727 & 115 & 20.4190341 & & 175th-85 \\
\hline CS & $00196 \mathrm{~B}$ & 11 & 2 & 158.5 & 1.58 & 45.2727 & 99.6 & 18.1352056 & 0.5 & 50 th- -75 \\
\hline IS & $00206 \mathrm{~B}$ & 12 & 1 & 153.5 & 1.53 & 60.9091 & 134 & 26.0195185 & & $2>95$ th \\
\hline SS & 00216B & 12 & 2 & 162.5 & 1.62 & 44.9091 & 98.8 & 17.1121365 & & 25 th- 50 \\
\hline DT & $00226 \mathrm{~B}$ & 12 & 1 & 155.375 & 1.55 & 39.7727 & 87.5 & 16.5547252 & -0.5 & 10th-25 \\
\hline RW & $00236 \mathrm{~B}$ & 12 & 1 & 165 & 1.65 & 54.0909 & 119 & 19.8681025 & & 50th-75 \\
\hline$B W$ & $00246 \mathrm{~B}$ & 12 & 2 & 148.5 & 1.48 & 39.3182 & 86.5 & 17.9502291 & & 25 th- 50 \\
\hline GZ & $00256 \mathrm{~B}$ & 12 & 1 & 142.125 & 1.42 & 33.1818 & 73 & \begin{tabular}{|l|}
16.4559701 \\
\end{tabular} & & 10 th-25 \\
\hline GR & $00156 \mathrm{~B}$ & 11 & 2 & 160 & 1.6 & 52.6136 & 115.75 & 20.5522017 & -0.5 & 55 th-85 \\
\hline GL & $00126 \mathrm{~B}$ & 11 & 2 & 147 & 1.47 & 37.5 & 82.5 & 17.3538803 & & 25 th- -50 \\
\hline$K C$ & $0016 \mathrm{~F}$ & 12 & 2 & 154.75 & 1.54 & 52.7273 & 116 & 22.2327849 & 1.5 & 55 th-90 \\
\hline TD & $0026 \mathrm{~F}$ & 12 & 1 & 156 & 1.56 & 63.1818 & 139 & 25.9622856 & & $2>95$ th \\
\hline ED & $0036 \mathrm{~F}$ & 11 & 2 & 146 & 1.46 & 39.1818 & 86.2 & \begin{tabular}{|l|}
18.3814122 \\
\end{tabular} & & 50 th-75 \\
\hline$M G$ & $0056 \mathrm{~F}$ & 12 & 2 & 153.3 & 1.533 & 41.1364 & 90.5 & 17.5041727 & -0.5 & 525 th- 50 \\
\hline $\mathrm{AH}$ & $0066 \mathrm{~F}$ & 12 & 1 & 142.25 & 1.42 & 46.8182 & 103 & 23.2186976 & 1.5 & 90th-95 \\
\hline AK & 00106 & 12 & 1 & 156 & 1.56 & 52.0455 & 114.5 & 21.3861993 & & 75th-85 \\
\hline $\mathrm{JM}$ & 00156 & 12 & 1 & 164.5 & 1.64 & 49.7727 & 109.5 & 18.5056244 & 0.5 & 50 th- -75 \\
\hline WM & 00166 & 12 & 1 & 160 & 1.6 & 47.9091 & 105.4 & 18.7144886 & 0.5 & 50 th-75 \\
\hline AO & 00206 & 12 & 2 & 163.5 & 1.63 & 47.8636 & 105.3 & \begin{tabular}{|l|}
18.014843 \\
\end{tabular} & & 25 th- 50 \\
\hline WP & 00216 & 12 & 1 & 145.5 & 1.45 & 41.8182 & 92 & 19.8897416 & 0.5 & 50 th-75 \\
\hline MR & 00236 & 12 & 2 & 153 & 1.53 & 37.7273 & 83 & \begin{tabular}{|l|}
16.1165674 \\
\end{tabular} & -0.5 & 510 th-25 \\
\hline CS & 00246 & 12 & 2 & 160 & 1.6 & 39.7727 & 87.5 & \begin{tabular}{|l|}
15.5362216 \\
\end{tabular} & -0.5 & 10 th-25 \\
\hline CW & 002564 & 11 & 1 & 160 & 1.6 & 56.3636 & 124 & 22.0170455 & & 585 th-90 \\
\hline
\end{tabular}




\begin{tabular}{|c|c|c|c|c|c|c|c|c|c|}
\hline Syptolit & Disst,Post Me & Num & Mumb & Total & Types of & Comment & 8 Consumpt & consumptio & Mesl 2 \\
\hline 110 & $50 \mid 00266$ & 1 & 0 & & Erape & & & & 00268 \\
\hline 95 & $\begin{array}{ll}66 & 00368\end{array}$ & & & & & & & & 00368 \\
\hline 91 & $60 \mid 00468$ & 1 & 0 & & appleno & & & & 00468 \\
\hline 79 & 5500768 & 0 & 0.25 & 0.25 & pickle & & & & 00768 \\
\hline 115 & $76 \mid 00968$ & & & & & & & & 10096 \\
\hline 99 & $7500116 \mathrm{~B}$ & 0 & 1 & \multicolumn{3}{|c|}{ 1| red peppers } & & & 001168 \\
\hline 111 & 80) $00146 \mathrm{~B}$ & 1 & 1 & \multicolumn{5}{|c|}{ 2| celery, peppers, cucumbers, tangerine } & 001468 \\
\hline 116 & $73 \mid 001668$ & 1 & 0 & & strawber & & & & $100166 \mathrm{~B}$ \\
\hline 103 & $64 / 001968$ & 1 & 0 & \multicolumn{4}{|c|}{ 1) strwerries, pineapple } & & 1001968 \\
\hline 112 & $53 \mid 00206 \mathrm{~B}$ & 2 & 0 & \multicolumn{3}{|c|}{ 2) apples grapes } & & & 1002068 \\
\hline 96 & 79002168 & & & & & & & & $00216 \mathrm{~B}$ \\
\hline 77 & $51 \mid 00226 \mathrm{~B}$ & & & & & & & & 002268 \\
\hline 106 & $0100236 \mathrm{~B}$ & 1] & 2 & \multicolumn{5}{|c|}{3 dried mango, fruit cochtail, lettuce arhod } & 1002368 \\
\hline 95 & $53 \mid 002468$ & 0 & 0 & 0 & & & & & $100246 \mathrm{~B}$ \\
\hline 90 & $72|00256 \mathrm{~B}|$ & 2 & 0 & & banana & medium & & & $100256 \mathrm{~B}$ \\
\hline 106 & $66 \mid 00156 \mathrm{~B}$ & & & & & & & & 1001568 \\
\hline 95 & $62|001268|$ & & & & & & & & 1001268 \\
\hline 110 & $81 \mid 0016 \mathrm{~F}$ & & & & & & & & $0016 \mathrm{~F}$ \\
\hline 1111 & $47 \mid 0026 \mathrm{~F}$ & 2 & 0 & & Erape & & 100 & & $0026 \mathrm{~F}$ \\
\hline 102 & $66 \mid 0036 \mathrm{~F}$ & 1 & 0 & \multicolumn{3}{|c|}{1 dementine } & 50 & 0.5 & $0036 \mathrm{~F}$ \\
\hline 90 & $520056 \mathrm{~F}$ & 1 & 0 & & nectarine & & 75 & 0.75 & $056 \mathrm{~F}$ \\
\hline 92 & $62 \mid 00665$ & 1.5 & 1 & 2.5 & carrots, $\mathrm{r}$ & arrots 2 & 3 & 0.075 & $0066 \mathrm{~F}$ \\
\hline 99 & $60 \mid 00106$ 月 & 0.5 & 0 & 0.5 & cramberi & fiber one & 100 & 0.5 & $\mid 00106$ \\
\hline 83 & 6500156 月 & 0.5 & 0 & 0.5 & Mriwber & & 100 & 0.5 & 00156 \\
\hline 90 & $61 \mid 0016$ 6月 & 15 & 0 & 1.5 & appleaz & se, clement & 100 & 15 & 00166 \\
\hline 103 & $53 \mid 00206$ 月 & 3 & 1 & & Erape, $\mathrm{c}$ & ate grapes & 75 & & 100206 \\
\hline 95 & $57 \mid 00216$ 月 & 1 & 1 & & arros, le & fiber one & 100 & & 00216 \\
\hline 92 & $73 \mid 00236$ 月 & 1 & 0 & & applenu & & 100 & & 10236 \\
\hline 96 & 6200246 月 & 1 & 0 & & apolera & & 100 & & 00246 \\
\hline 95 & $73 \mid 00256 f$ & 1 & 0 & & appleav & & 100 & & 10256 \\
\hline
\end{tabular}




\begin{tabular}{|c|c|c|c|c|c|c|c|c|c|c|c|c|c|}
\hline AVGVEG & AVG NUMB & Ang of o 5 surve 0 & $\mathrm{Malc}$ & $22 \mathrm{~A}$ & $=1, A=$ & Q3A & Alot & Q5 & 56. & 27 & BA & 09 & \\
\hline 0 & 1 & 0.800268 & 1 & 3 & & 4 & 3 & 3 & 4 & 2 & 2 & & 3 \\
\hline 0. & 0.5 & 10036 畐 & 1 & 3 & & 4 & 4 & (4) & 4 & 4 & s. & & 4 \\
\hline 0 & 1 & $100466^{6}$ & 1 & 3 & & 4 & & 4 & 4 & 4 & 7 & & 4 \\
\hline 0.125 & 0.125 & 0 00768 & 1 & 3 & & 0 & 4 & 3 & 3 & 3 & 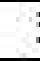 & & 3 \\
\hline 0) & 1.5 & 300966 & 2 & 3 & & 4 & 3 & 3 & 4 & 3 & 3 & & 4 \\
\hline 0.5 & 125 & 15 |0011日 & 1 & 3 & & 4 & 4 & 3 & 4 & 4 & 7 & & 4 \\
\hline 0.75 & 2375 & $2.75 \mid 00144$ & 2 & 3 & & 4 & 3 & 3 & 3 & 2 & - & & 3 \\
\hline 0 & 125 & 1.500168 & 2 & 3 & & 4 & 4 & 4 & 4 & 4 & t & & 4 \\
\hline 0 & 1.5 & 2 Co19月 & 2 & 3 & & 4 & 3 & 3 & 3 & 2 & 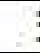 & & 3 \\
\hline 0 & 1.5 & 10020 臬 & 1 & 3 & & 4 & 3 & 2 & 3 & 2 & 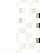 & & 4) \\
\hline 0 & 0. & 000218 & 2 & 3 & & 4 & 3 & 4 & 3 & 3 & 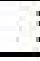 & & 4 \\
\hline of & 0.75 & $15 \mid 00228$ & 1 & 3 & & 4. & 3 & 2 & 1 & 2 & i & & 2 \\
\hline & & 002368 & & & & & & 1 & & 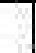 & & & \\
\hline 0 & 0.5 & 100248 & 2 & 3 & & 4 & 4 & 2 & 4 & 3] & the & & 3 \\
\hline 0. & 1.5 & 100256 & 1 & 3 & & o) & 0 & 3 & 2 & 3 & t & & 2 \\
\hline 0 & 1.5 & 3.0015 & 2 & 3 & & 4 & 3 & 2 & 3 & 3 & 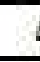 & & 3 \\
\hline 0 & 0) & $0 \mid 0012 \mathrm{~A}$ & 2 & 3 & & 4 & 4 & 2 & 4 & 3 & 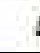 & & 4 \\
\hline 0 & 0 & 011 . & 2 & 3 & & 4 & 3 & 3 & 2 & 2 & 2 & & 4 \\
\hline 0) & 1.5 & $1.5002 \mathrm{~B}$ & 1 & 3 & & 4. & 4 & 3 & 4 & 4 & te & & 4 \\
\hline 0 & 1 & 03750036 & 2 & 3 & & 4 & 3 & 3 & 4 & 3 & 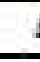 & & 4 \\
\hline 0 & 1.625 & $0.375 \mid 05$ A & 2 & 3 & & 4 & 4 & 4 & 4 & 4 & 2 & & 4) \\
\hline 0.5 & 1.5 & $0.0375 \mid 0061$ & 1 & 3 & & 4 & 4 & 3 & 3 & 4 & 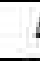 & & 4 \\
\hline 0 & 0.75 & $0.75|0010|$ & 1 & 3 & & 4 & 3 & 3 & 4 & 4 & 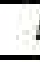 & & 4 \\
\hline 0 & 0.75 & $0.75|0015|$ & 1 & 2 & & 4 & 4 & 3 & 4 & 4 & 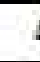 & & 3 \\
\hline 의 & 1.25 & $1.25|0016|$ & 1 & 3 & & 4 & 4 & 3 & 4 & 3. & 2 & & 4 \\
\hline 0.5 & 3] & $2.5|020|$ & 2 & 3 & & 4 & 4 & 3 & 4 & 3 & 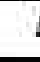 & & 4 \\
\hline 1 & 2.25 & $2.25|0021|$ & 1 & 3 & & 4 & 4 & 3 & 4 & 4 & 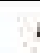 & & 4 \\
\hline 0 & 1 & $1|023|$ & 2 & 3 & & 3 & 3 & 2 & 2 & 1) & 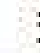 & & 3 \\
\hline 0. & 1 & $1|0024|$ & 2 & 6 & & 3 & 3 & 4 & 2 & 2 & 3 & & 2 \\
\hline 0 & 0.5 & $0.5|0025|$ & 1 & 3 & & 3 & 3 & 3 & 3 & 3 & 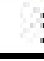 & & 3 \\
\hline
\end{tabular}




\begin{tabular}{|c|c|c|c|c|c|c|}
\hline Number of $\mathrm{Fl}$ & Number of & Total Mumbs & Types of sen & $\gamma$ consumpt & est consumg dc & AVGFRUI \\
\hline 1 & 0 & 1 & apple & 60 & 0.8 & 1 \\
\hline 1 & 0 & & banana & 100 & 1 & 0.5 \\
\hline 1 & 0 & & applenuce & 100 & 1 & 1 \\
\hline 0 & 0 & & none & 0 & 0 & 0 \\
\hline 3 & 0 & & applesauce, & 0 & of & 1.5 \\
\hline 1.5 & 0 & 1.5 & large spple & 75 & 1125 & 0.75 \\
\hline 2.25 & 0.5 & 2.75 & tomato salsa & 70 & $1925 \mathrm{a}$ & 1.625 \\
\hline 1.5 & 0 & 1.5 & apple slices, & 100 & 1.5 & 1.25 \\
\hline 2 & 0 & & strowberries & 100 & 2 & 1.5 \\
\hline 1 & 0 & & mand ornis: & 100 & 1 & 1.5 \\
\hline 0 & 0 & & none & 0 & 0 & 0 \\
\hline 1.5 & 0 & 1.5 & applenuce, & 100 & 1.5 & 0.75 \\
\hline 1 & 2 & & Peppers, car & 75 & 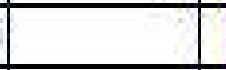 & \\
\hline 1 & 0 & & organic fortit & 100 & 1]d & 0.5 \\
\hline 1 & 0 & & mandarin or: & 100 & 1 & 1.5 \\
\hline 3 & 0 & & $3 / 4$ cup of $n$ & 100 & 3) & 1.5 \\
\hline 0 & 0 & & none & 0 & 0. & 0 \\
\hline 0 & 0 & & none & & 0 & 0 \\
\hline 1 & 0 & & clementine & 100 & 1 & 1 \\
\hline 1 & 0 & & apple & 25 & 0.25 & 0.5 \\
\hline 2.25 & 0 & 2.25 & cherries, nec & 0 & of & 0.5 \\
\hline 0.5 & 0 & & clementine 1 & 0 & 0 & 125 \\
\hline 1 & 0 & & mandarin of & 100 & 1 fi & 0.25 \\
\hline 1 & 0 & & strwberries & 100 & 1 & 0.25 \\
\hline 1 & 0 & & applemuce, & 100 & 10 & 0.75 \\
\hline 2 & 0 & & grapes, risir & 100 & 2 & 2 \\
\hline 1.5 & 1 & & fresh strowb & 100 & $2.5=$ & 1 \\
\hline 1 & 0 & & applemuce & 100 & 1 & 0.5 \\
\hline 1 & 0 & & applesauce & 100 & $1 \mathrm{fi}$ & 0.5 \\
\hline 0 & 0 & & none & & 0 & 0.5 \\
\hline
\end{tabular}




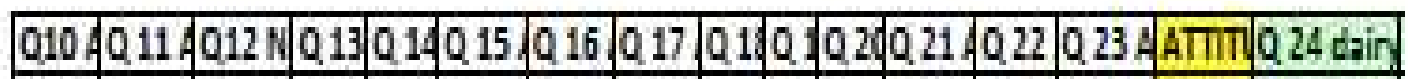

\begin{tabular}{|c|c|c|c|c|c|c|c|c|c|c|c|c|c|c|}
\hline 3 & 4 & 3 & & 3. & 3 & 4 & 3 & 42 & & & 4 & 3. & 67 & \\
\hline 4) & 4 & 3) & 3 & 3. & 3 & 3 & 3 & \begin{tabular}{l|l}
3 & 3 \\
\end{tabular} & & 3. & 3 & I & 70 & \\
\hline 4 & 4 & 4 & 4 & 3 & 3 & (4) & 3 & \begin{tabular}{l|l|l}
4 \\
\end{tabular} & 4.4 & 3 & 4 & 3 & 79 & \\
\hline 3) & 4 & 4 & 4 & 3. & 3 & 3) & 3 & $\begin{array}{lll}33 & 3\end{array}$ & \begin{tabular}{l|l}
3 & 3
\end{tabular} & ] & 4 & 3. & 65 & \\
\hline 4 & 3. & 3 & 4 & 3 & 3 & 3 & 3 & \begin{tabular}{l|l}
3 & 3
\end{tabular} & 34 & 4 & 3 & 2 & 69 & \\
\hline 4 & 4. & 2 & 3 & 3) & 3 & 3) & 3 & 22 & 22 & 2 & 2 & 2 & 64 & \\
\hline 2 & 4) & 3 & 3 & 4 & 3 & 3 & 3 & 23 & 35 & 2 & 4 & 3 & 64 & \\
\hline 3] & 4 & 5 & 4 & 4. & 4 & 3 & 3 & $\begin{array}{ll}3 & 4 \\
\end{array}$ & 4 & 3] & 4 & 3 & 79 & \\
\hline 3 & 2. & 5 & 4 & 3 & 3 & 3 & 2 & 26 & 42 & 4 & 4 & 4 & 68. & \\
\hline 3 & 4 & 3 & 3 & 2 & 1 & 2 & 2 & $1:$ & 34 & 1 & 4 & 3 & 57 & \\
\hline 4 & 4 & 5 & 2 & 2 & 3 & 2 & 2 & 22 & 24 & 3] & 2 & 2 & 63 & \\
\hline 3 & 4 & 1 & 3 & 2 & 2 & 3 & 2 & 22 & 212 & 3 & 3 & 1 & 49 & \\
\hline & & & & & & & & & & & & & & \\
\hline 1 & 4) & 3 & 2 & 2 & 3 & 4 & 2 & 33 & 32 & 1 & 3 & 2 & 57 & \\
\hline 4 & 1 & 2 & 3 & 3 & 3 & 4 & 3 & $3:$ & 13 & 2 & 3 & 1 & 50 & \\
\hline 3 & 4 & 3 & 3 & 3 & 3 & 3 & 3 & 23 & 32 & 2 & 3 & 2 & 61 & \\
\hline 3 & 3 & 4 & 2 & 2 & 2 & 2 & 2 & 2 & 2 & 2 & 2 & 3 & 59 & \\
\hline 3 & 4 & 3 & 3 & 3 & 3 & 4 & 3 & 22 & $4 \sqrt{4}$ & 2 & 3 & 3. & 64 & \\
\hline 4 & 4 & 3 & 3 & 3 & 3 & 3 & 3 & $\begin{array}{l:}3 \\
3\end{array}$ & $\begin{array}{l}3 / 4 \\
3\end{array}$ & 4 & 4 & 3 & 74 & \\
\hline 3 & 4 & 3 & 2 & 3 & 2 & 2 & 2 & 23 & $\sqrt[3]{3}$ & 3 & 3 & 3 & 63 & \\
\hline 4 & 4 & 5 & 4 & 4 & 4 & 4 & 4 & 4 & 4. & 4 & 4 & 3 & 84 & \\
\hline 4 & 3 & 5 & 2 & 3) & 2 & 1 & 2 & 20 & 0 & 2 & 2 & 3 & 59 & \\
\hline 4 & 4 & 4 & 3. & 3 & 3 & 3 & 74 & $\sqrt[4]{4}$ & 4 & 4 & (4) & 司 & 77 & \\
\hline 2 & 4 & 1 & 3 & 3 & 3 & 3] & 3 & 3 & $\begin{array}{lll}3 & 2\end{array}$ & 2 & 3 & 1 & 62 & \\
\hline 4 & 4 & 3 & 3 & 3 & 3 & 7 & 3 & 3. & 4 & 4 & 4 & 3 & 74 & \\
\hline 4 & 4 & 3] & 4 & 3] & 3) & 4 & 3 & 3] & 4) 6 & 4 & 4 & 4 & 77 & \\
\hline 4 & 4 & 2 & 3 & 3 & 3 & 3 & 3 & $\begin{array}{ll}3 \\
\end{array}$ & $\sqrt[3]{3}$ & 3 & 3 & 3. & 70 & \\
\hline 2 & 4 & 3 & 2 & 2 & 1 & 2 & 3 & 1 & 212 & 2 & 3 & 3 & 49 & \\
\hline 2 & 4 & 5 & 4 & 4. & 3 & 3 & 3 & 3 & \begin{tabular}{l|l}
3 & 3 \\
\end{tabular} & 3) & 4 & 4 & 67] & \\
\hline 3 & 4 & 3 & 3] & 3 & 3 & 3 & 3 & 3 . & 3) & 3 & 3 & 3 & 64 & \\
\hline
\end{tabular}




\begin{tabular}{|c|c|c|c|c|c|c|c|c|c|c|c|c|c|c|c|}
\hline $25 \mathrm{~d}$ & 22 & & 2281 & & & Q31to & Q32A & Q33 & Q37? & KONOWLE & Q34 X & $35, \mathrm{C}$ & 36 & 3811 & 391 \\
\hline 2 & 3 & 1 & 1 & 2 & 3 & 3 & 3 & 1 & 3 & 11 & 1 & 1 & 0 & 4 & 3 \\
\hline 2 & 1 & 1 & 1 & 2 & 3) & 3 & 3 & 1 & 3 & 10 & 1 & 2 & 0 & 4 & 4 \\
\hline 2 & 3 & 2 & 1 & 2 & $\sqrt{3}$ & 3 & 3 & 1 & 3 & 11 & 1 & 1 & 1 & 4 & 4 \\
\hline 2 & 3 & 1 & 1 & 2 & 3 & 3 & 3 & 1 & 3 & 10 & 1 & 1 & 1 & 4 & 4 \\
\hline 2 & 4 & 1 & 1 & 2 & 3 & 3 & 3 & 1 & 3 & 10 & 1 & 1 & 1 & 4 & 4 \\
\hline 2 & 1 & 1 & 1 & 2 & 3 & 3 & 3 & 1 & 1 & 9 & 1 & 1 & 2 & 4 & 4 \\
\hline 2 & 3 & 1 & 1 & 2 & 3 & 3 & 3 & 1 & 3 & 11 & 2 & 2 & 1 & 4 & 4 \\
\hline 2 & 3 & 1 & 1 & 2 & 3 & 3 & 3 & 1 & 3 & & 2 & 2 & 2 & 4 & 4 \\
\hline 1 & 2 & 1 & 1 & 2 & 3 & 3 & 3 & 1 & 3 & & 1 & 2 & 1 & 4 & 4 \\
\hline 2 & 3 & 1 & 1 & 2 & 3 & 3 & 3 & 1 & 3 & 11 & 1 & 1 & 0 & 4 & 4 \\
\hline 2 & 3 & 1 & 1 & $\sqrt{2}$ & 3 & 3 & 3 & 1 & 3 & 11 & 1 & 1 & 0 & 4 & 4 \\
\hline 2 & 3 & 1 & 1 & 2 & 3 & 3 & 3 & 1 & 1 & 10 & 0 & 1 & 0 & 4 & 4 \\
\hline & & & & & & & & & & & & & & & \\
\hline 2 & 3 & 1 & 1 & 2 & 3 & 1 & 3 & 1 & 3 & 10 & 1 & 2 & 1 & 3 & 4 \\
\hline 2 & 5 & 1 & 2 & 2 & 3 & 1 & 2 & 1 & 0 & 7 & 1 & 1 & 0 & 0 & 2 \\
\hline 2 & 3 & 1 & 1 & 2 & 3 & 3 & 3 & 1 & 3 & 11 & 1 & 2 & 1 & 4 & 4 \\
\hline 2 & 3 & 1 & 1 & 2 & 3 & 3 & 3 & 3 & 3 & 10 & 1 & 0 & 0 & 4 & 3 \\
\hline 2 & 5 & 1 & 1 & 2 & 3 & 4 & 4 & 4 & 1 & 6 & 1 & 1 & 1 & 4 & 4 \\
\hline 2 & 5 & 1 & 0 & 2 & 3 & 4 & 1 & 2 & 1 & 5 & 2 & 0 & 0 & 4 & 4 \\
\hline 2 & 3 & 1 & 1 & 2 & 3 & 1 & 3 & 1 & 1 & 9 & 0 & 1 & 1 & 3 & 4 \\
\hline 2 & 3 & 1 & 1 & 2 & 3 & 3 & 3 & 1 & 3 & 11 & 2 & 2 & 2 & 4 & 4 \\
\hline 2 & 5 & 1 & 1 & 2 & 3 & 3 & 3 & 1 & 3 & 10 & 1 & 1 & 0 & 4 & 4 \\
\hline 2 & 5 & 1 & 1 & 2 & 3 & 1 & 3 & 3 & 1 & 7 & 1 & 2 & 1 & 4 & 4 \\
\hline 2 & 5 & 1 & 1 & 2 & 3 & 3 & 3 & 1 & 1 & 9 & 1 & 2 & 0 & 4 & 2 \\
\hline 2 & 5 & 1 & 4 & 2 & 3 & 3 & 4 & 4 & 3 & 7 & 1 & 1 & 1 & 4 & 4 \\
\hline 2 & 3 & 1 & 1 & 2 & 3 & 1 & 3 & 1 & 1 & 9 & 2 & 2 & 1 & 4 & 4 \\
\hline 2 & 2 & 1 & 1 & 2 & 3 & 1 & 2 & 1 & 1 & 7 & 2 & 1 & 1 & 3 & 4 \\
\hline 2 & 1 & 1 & 1 & 2 & 3 & 3 & 3 & 1 & 1 & 9 & 1 & 0 & 0 & 3 & 3 \\
\hline 2 & 1 & 1 & 1 & 2 & 3 & 3 & 2 & 4 & 1 & 7 & 1 & 1 & 2 & 3 & 3 \\
\hline 2 & 5 & 1 & 4 & 2 & 3 & 1 & 4 & 3 & 1 & 5 & 1 & 1 & 1 & 4. & 4 \\
\hline
\end{tabular}




\begin{tabular}{|c|c|c|c|c|c|c|c|c|c|c|c|c|c|c|c|c|c|c|}
\hline 40110 & $4 \|$ & 042 & 3 & PERCEPT & 944 & $d 0$ & 51 & Q45 I & Q47 & 04 & 10 & II & & & 5 & 5310 & 54 & 1551 \\
\hline 3 & 4 & 4 & 4 & 24 & & 4 & 4 & 5 & 4 & & 5 & & 5 & 5 & 4 & 5 & 4 & 3 \\
\hline 4 & 4 & 4 & 4 & 27 & & 3 & 5 & 4 & 4 & & & & 4 & 4 & 4 & 4 & 4 & 4 \\
\hline 4 & 4 & 4 & 4 & 27 & & 3 & 5 & 4 & 5 & & 5 & & 司 & 5 & 5 & \begin{tabular}{l|}
5 \\
\end{tabular} & 5 & 5 \\
\hline 4 & 4 & 4 & 4 & 27 & & 4 & 5 & 5 & 5 & & 5] & & 司 & 3) & 5 & 5 & 4 & 5 \\
\hline 4 & 4 & 4 & 3 & 26 & & 4 & 5 & 4 & 5 & & 2 & & 司 & 5 5. & 5. & 5 & 5 & 4 \\
\hline 4 & 4 & 4 & 4 & 28 & & 3) & 5 & 5 & 3 & & 5 & & 司 & 5 & 5 & 5 & 5 & 5 \\
\hline 4 & 4 & 4 & 4 & 29 & & 4 & 5 & 5 & 5 & & 5 & & 司 & 5 & 5 & 5 & 5 & 5 \\
\hline 4 & 4 & 4 & 4 & 30 & & 5 & 5 & 5 & 3 & & 5 & & 5 & 5 & 5 & 5 & 5 & 5 \\
\hline 4 & 4 & 4 & 4 & 20 & & 2 & 5 & 1 & 2 & & 5 & & 司 & 4 & 5 & 5 & 5 & 5 \\
\hline 4 & 4 & 4 & 4 & 26 & & 4 & 5 & 5 & 5 & & 5 & & 5 & 5 & 5 & 5 & 5 & 4 \\
\hline 4 & 4 & 4 & 4 & 26 & & 2 & 5 & 4 & 1 & & 5 & & 5 & 5 & 5 & 5 & 5 & 5 \\
\hline 3 & 3 & 4 & 3 & 22 & & 1] & 5 & 4 & 5 & & 5 & & 5 & 5 & 4 & 4 & 4 & $4 \mid$ \\
\hline & & & & & & & & & & & & & & & & & & \\
\hline 2 & 4 & 2 & 4 & 23 & & 2 & 4 & 4 & 2 & & 5 & & 司 & 3) & 4 & 4 & 5 & 4 \\
\hline 3 & 4 & 4 & 4 & 19 & & 4 & 5 & 4 & 2 & & j. & & 司 & 4 & 4 & 4 & 3 & 4 \\
\hline 4 & 4 & 4 & 4 & 28 & & 3 & 5 & 4 & 3 & & 5 & & 司 & 4 & 5 & 5 & 5 & 5 \\
\hline 3 & 4 & 3 & 4 & 22 & & 3. & 5 & 5 & 5 & & 5 & & 5 & 5 & 5 & 5 & 5 & 5 \\
\hline 3 & 4 & 3 & 3 & 24 & & 3 & 5 & 3 & 2 & & 5 & & 司 & 3 | & 4 & 4 & 3 & 3) \\
\hline 4 & 4 & 4 & 4 & 26 & & 4 & 5 & 5 & 5 & & 5 & & 5 & 5 & 5 & 5 & 5 & 5 \\
\hline 4 & 4 & 4 & 4 & 25 & & 1 & 5 & 5 & 5 & & 5 & & 司 & 4 & 4 & 5 & 5 & 5 \\
\hline 4 & 4 & 4 & 4 & 30 & & 4 & 5 & 5 & 5 & & 5 & & 5 & 4 & 5 & 5 & 5 & 5 \\
\hline 3 & 3 & 4 & 4 & 24 & & 4 & 5 & 4 & 3 & & 5 & & 司 & 5 & 5 & 5 & 5 & 3 \\
\hline 4 & 4 & 4 & 4 & 28 & & 4 & 5 & 5 & 5 & & 5 & & 司 & 5 & 5 & 5 & 5 & 5 \\
\hline 4 & 3 & 4 & 3 & 23 & & 3 & 5 & 5 & 5 & & 5 & & 司 & 5 & 3 & 2 & 5 & 4 \\
\hline \begin{tabular}{l|l}
4 \\
\end{tabular} & 4 & 4 & 4 & 27 & & 3 & 4 & 3 & 4 & & 5. & & 5 & 4 & 5 & 5 & 5 & 5 \\
\hline 4 & 4 & 4 & 2 & 27 & & 5 & 5 & 5 & 5 & & & & 5 & 5 & 5 & 5 & 1 & 3) \\
\hline 4 & 4 & 4 & 2 & 27 & & 3 & 5 & 5 & 3 & & & & 5 & 4 & 5 & 5 & 5 & 4 \\
\hline 2 & 4 & 4 & $\vdots$ & 20 & & 3 & 5 & 4 & 5 & & 5 & & 5 & 5 & 4 & 5 & 5 & 3) \\
\hline 4 & 3 & 4 & 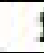 & 22 & & 5 & 5 & 5 & 5 & & & & 司 & 5 & 5 & 5 & 2 & 1 \\
\hline 4 & 4 & 4 & 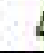 & 27 & & 2 & 4 & 4 & 3 & & & & 4 & 5 & 5 & 5 & 5 & 5 \\
\hline
\end{tabular}




\begin{tabular}{|c|c|c|c|c|c|c|c|c|c|c|c|c|c|c|c|c|}
\hline $056 \mathrm{~s}$ & Q56. & 256 & Q56: & 250 & 056 & FRUT & FruI & Q56d & Q560 & 05 & Q56 & POTATI & POTA & Q56f & Q56f1 & 056 \\
\hline 1 & 2 & 1 & 1 & 1 & 2 & 1 & 1.66 & 0 & 2 & 0 & 2 & 0 & & 1 & 1 & 0 \\
\hline 1 & 1 & 0 & 0 & 0 & 0 & 0.33 & 0.33 & 0 & 0 & 0 & 0 & 0 & & 0 & 0 & 0 \\
\hline 1 & 2 & 1 & 2 & 1 & 2 & 1 & 2 & 1 & 2 & 0 & 0 & 0.5 & 1 & 1 & 3 & 0 \\
\hline 1 & 1 & 0 & 0.5 & 1 & 2 & 0.66 & 1.16 & 1 & 3 & 0 & 1 & 0.5 & 2 & 0 & 1 & 1 \\
\hline 1 & 0.5 & 1 & 0.5 & 1 & 0.5 & 1 & 0.5 & 0 & 0 & 0 & 0 & 0) & & 0 & 0 & 0 \\
\hline 1 & 1 & 0 & 0 & 1 & 2 & 0.66 & 1 & 0 & 0 & 0 & 0 & 0 & & 0 & 0 & 0 \\
\hline 1 & 2 & 0 & 0 & 1 & 1 & 0.66 & 1 & 0 & 0 & 0 & 0 & 0 & & 0 & 0 & 1 \\
\hline 1 & 2 & 1 & 1 & 1 & 2 & 1 & 1.66 & 0 & 1 & 0 & 1 & of & & 1 & 3 & 0 \\
\hline 1 & 2 & 1 & 1 & 1 & 2 & 1 & 1.66 & 0 & 0 & 0 & 0 & 0 & & 1 & 2 & 0 \\
\hline 1 & 1 & 0 & 0 & 1 & 2 & 0.66 & 1 & 0 & 0 & 0 & 0 & 0 & & 0 & 0 & 0 \\
\hline 1 & 1 & 0 & 0 & 1 & 2 & 0.66 & 1 & 1 & 3 & D. & 0 & 0.5 & 1.5 & 1 & 3 & 1 \\
\hline 1 & 1 & 1 & 1 & 1 & 1 & 1 & 1 & 0 & 0 & 0 & 0 & of & & 1 & 2 & 0 \\
\hline & & & & & & & & & & 0 & & & & & & \\
\hline 1 & 2 & 0 & 1 & 0 & 2 & 0.33 & 1.66 & 0 & 1 & 0 & 1 & 0) & & 0 & 1 & 1 \\
\hline 1 & 0.5 & 0 & 0 & 1 & 1 & 0.66 & 0.5 & 0 & 0 & 0 & 0 & 0 & & 1 & 2 & 0 \\
\hline 0 & 0 & 0 & 0 & 1 & 1 & 0.33 & 0.33 & 0 & 0 & 이 & 0 & 0 & & 0 & 0 & 1 \\
\hline 0 & 0 & 1 & 1 & 1 & 1 & 0.66 & 0.66 & 0 & 0 & 0 & 0 & of & & 0 & 0 & 1 \\
\hline 1 & 2 & 0 & 0 & 0 & 0 & 0.33 & \begin{tabular}{|l|}
0.66 \\
\end{tabular} & 1 & 1 & 1 & 3 & 1 & 2 & 1 & 2 & 1 \\
\hline 1 & 1 & 1 & 1 & 0 & 0 & 133 & 0.65 & 1 & 2 & 0 & 0 & 0.5 & 1 & 1 & 2 & 1 \\
\hline 1 & 0.5 & 0 & 0 & 1 & 2 & 0.66 & 0.83 & 0 & 0 & 0 & 0 & 0 & 0 & 0 & 요 & 0 \\
\hline 1 & 2 & 0 & 1 & 1 & 0.5 & 0.66 & 1.16 & 0 & 0 & 0 & 0 & 0) & 0 & 0 & o & 0 \\
\hline 1 & 2 & 0 & 0 & 1 & 1 & 0.66 & 1 & 0 & 0 & 0 & 0 & 0 & 0 & 0 & 0 & 0 \\
\hline 1 & 1 & 1 & 1 & 1 & 1 & 1 & 1 & 0 & 0 & 1 & 2 & 0.5 & 1 & 1 & 1 & 1 \\
\hline 0 & 0 & 0 & 0 & 1 & 1 & 0.33 & 0.33 & 0 & 0 & 1 & 2 & 0.5 & 1 & 0 & 0 & 0 \\
\hline 1 & 2 & 1 & 0.5 & 1 & 1 & 1 & 1.16 & 0 & 0 & 0 & 1 & of & 0.5 & 1 & 1 & 0 \\
\hline 1 & 2 & 1 & 2 & 0 & 0 & 0.66 & 133 & 0 & 0 & 0 & 0 & 0 & 0 & 0 & 0 & 0 \\
\hline 1 & 1 & 1 & 2 & 1 & 2 & 1 & 1.66 & 0 & 1 & 0 & 0 & 0 & 0.5 & 0 & 0. & 1 \\
\hline 1 & 1 & 0 & 0 & 1 & 2 & 0.66 & 1 & 1 & 3 & 0 & 0 & 0.5 & 1.5 & 1 & 3 & 0 \\
\hline 1 & 0.5 & 1 & 1 & 0 & g & 0.66 & 05 & 0 & 0 & 0) & 0 & 0 & 0 & 0 & 요 & 0 \\
\hline 1 & 1 & 0 & 0 & 1 & 2 & 0.66 & 1 & 0 & 0 & 0 & o & 0. & 0 & 1 & 2 & 0 \\
\hline
\end{tabular}




\begin{tabular}{|c|c|c|c|c|c|c|c|c|c|c|c|c|c|c|c|}
\hline 6 & 5 & a56h & & 059 & $156 \mathrm{~J}$ & Q56 & Q56h & Q56k & \begin{tabular}{|l|} 
VEGEEI \\
\end{tabular} & RELATIVE & Q56LY Yo & 056 - & $1056 n$ & Q56n & \\
\hline 2 & 0 & 0.5 & 1 & 1 & 1 & 1 & 1 & 2 & 0.66 & 1.25 & 1 & 2 & 0 & & 1 \\
\hline 0 & $\overline{1}$ & 1 & $\overline{0}$ & 0 & 0 & 0 & 1 & 3 & 0.33 & 0.66 & 0 & 0 & 0 & & 0 \\
\hline 0 & 0 & 0 & 0 & 0 & 1 & 3 & 1 & 7 & 0.5 & 1.33 & 1 & 3 & 1 & & 2 \\
\hline 3 & 0 & 0.25 & 0 & 1 & 1 & 3 & 1 & 2 & 0.5 & 1.7 & 0 & 1 & 0 & & 1 \\
\hline 0$]$ & $\overline{0}$ & 0 & 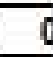 & 0 & 1 & 1 & 0 & 0 & 0.16 & 0.16 & 0 & 이 & 의 & & 0 \\
\hline 0 & $\overline{0}$ & 0 & 1 & 2 & 0 & 0 & 1 & 2 & 0.33 & 0.66 & 1 & 3 & 0 & & 0 \\
\hline $3 \mid$ & 0 & 0 & 1 & 3 & 1 & 2 & 의 & 0 & 0.5 & 1.33 & 0 & 이 & 0 & & 0 \\
\hline 1 & 1 & 0.5 & 1 & 3 & 0 & 1 & 1 & 2 & 0.66 & 1.75 & 1 & 3 & 1 & & 3 \\
\hline 0 & 0 & 0 & 1 & 3 & 1 & 3 & 이 & 의 & 0.5 & 1.33 & 0 & 0 & 0 & & 0 \\
\hline 0 & 0 & 0 & 0 & 1 & 1 & 1 & 0 & 0 & 0.166 & 0.33 & 0 & 이 & 0 & & 0 \\
\hline 2 & 0 & 0 & 0 & 0 & 0 & 0 & 0 & 0 & 0.33 & 0.533 & 0 & 0 & 0 & & 0 \\
\hline 0 & 0 & 0 & 0 & 0 & 1 & 2 & 0 & 0 & 0.33 & 0.66 & 0 & o & 0 & & 0 \\
\hline & & & & & & & & & & & & & & & \\
\hline 2 & $\overline{0}$ & 1 & 10 & 2 & 1 & 1 & 0 & 2 & 0.33 & 15 & 0 & 2 & 1 & & 1 \\
\hline 0 & 0 & 0 & 0 & 0 & 1 & 1 & 0 & 이 & 0.33 & 05 & 0 & 이 & 0 & & 0 \\
\hline 3) & 0 & 0 & 10 & 0 & 1 & 2 & 0 & 0 & 0.33 & 0.83 & 0 & 0 & 0 & & 0 \\
\hline 3 & 0 & 0.25 & 0 & 0 & 1 & 1 & 0 & 3 & 0.33 & 1.2 & 1 & 2 & 0 & & 0 \\
\hline 3 & 0 & 0 & 1 & 2 & 0 & 0 & 0 & 0 & 0.5 & 1.16 & 0 & 0 & 0 & & 0 \\
\hline 1 & 1 & 1 & 0 & 0 & 0 & 0 & 0 & 0 & 0.5 & 0.66 & 0 & 0] & 0 & & 0 \\
\hline 0 & 0 & 0 & 0 & 0 & 1 & 2 & 0 & 0 & 0.16 & 0.33 & 0 & 이 & 0 & & 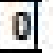 \\
\hline 2 & 0 & 0 & 0 & 0 & 0 & 3 & 1 & 2 & 1.16 & 1.16 & 0 & 이 & 0 & & 0 \\
\hline 0 & $\overline{0}$ & 0 & 7 & 0 & 0 & 可 & 1 & 0 & 0.16 & 0 & 0 & 0 & 1 & & $\overline{0}$ \\
\hline 31 & $\overline{1}$ & 0.5 & 1 & 2 & 1 & 3 & 1 & 1 & 1 & 1.75 & 1 & 1) & 1 & & 3 \\
\hline 0 & 0 & 0 & 1 & 2 & 0 & 0 & 0 & 0 & 0.16 & 0.33 & 0 & 이 & 0 & & 0 \\
\hline 0 & 0 & 0 & 1 & 1 & 1 & 2 & 0 & 0 & 0.5 & 0.66 & 0 & 1 & 1 & & 2 \\
\hline 0 & 0 & 0 & 0 & 0 & 0 & 0 & 0 & o] & of & 0 & 0 & 이 & 0 & & 0 \\
\hline 1 & 0 & 0 & 0 & 0 & 1 & 2 & 0 & 0 & 0.33 & 0.5 & 0 & 0 & 0 & & 0 \\
\hline 0 & 0 & 0 & 0 & 0 & 0 & 0 & 0 & 0 & 0.16 & 0.5 & 0 & 0 & 0 & & 0 \\
\hline 0 & 0 & 0 & 0 & 0 & 1 & 3 & 0 & 0 & 0.16 & 0.5 & 이 & 0 & 0 & & 0 \\
\hline 0 & 0 & 0 & 1 & 3 & 1 & 2 & 0 & 0 & 0.5 & 116 & 1 & 2 & 0 & & 0 \\
\hline
\end{tabular}




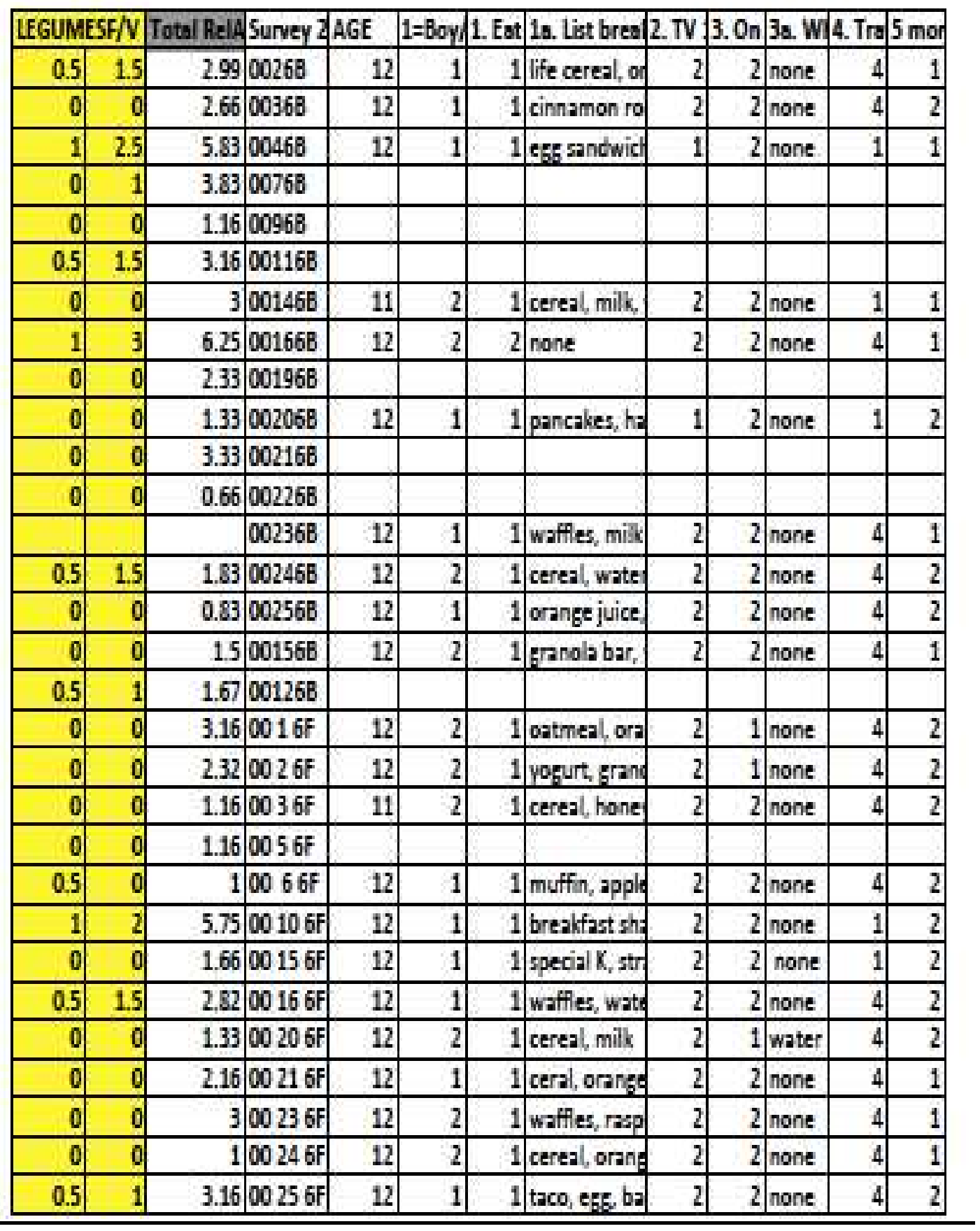




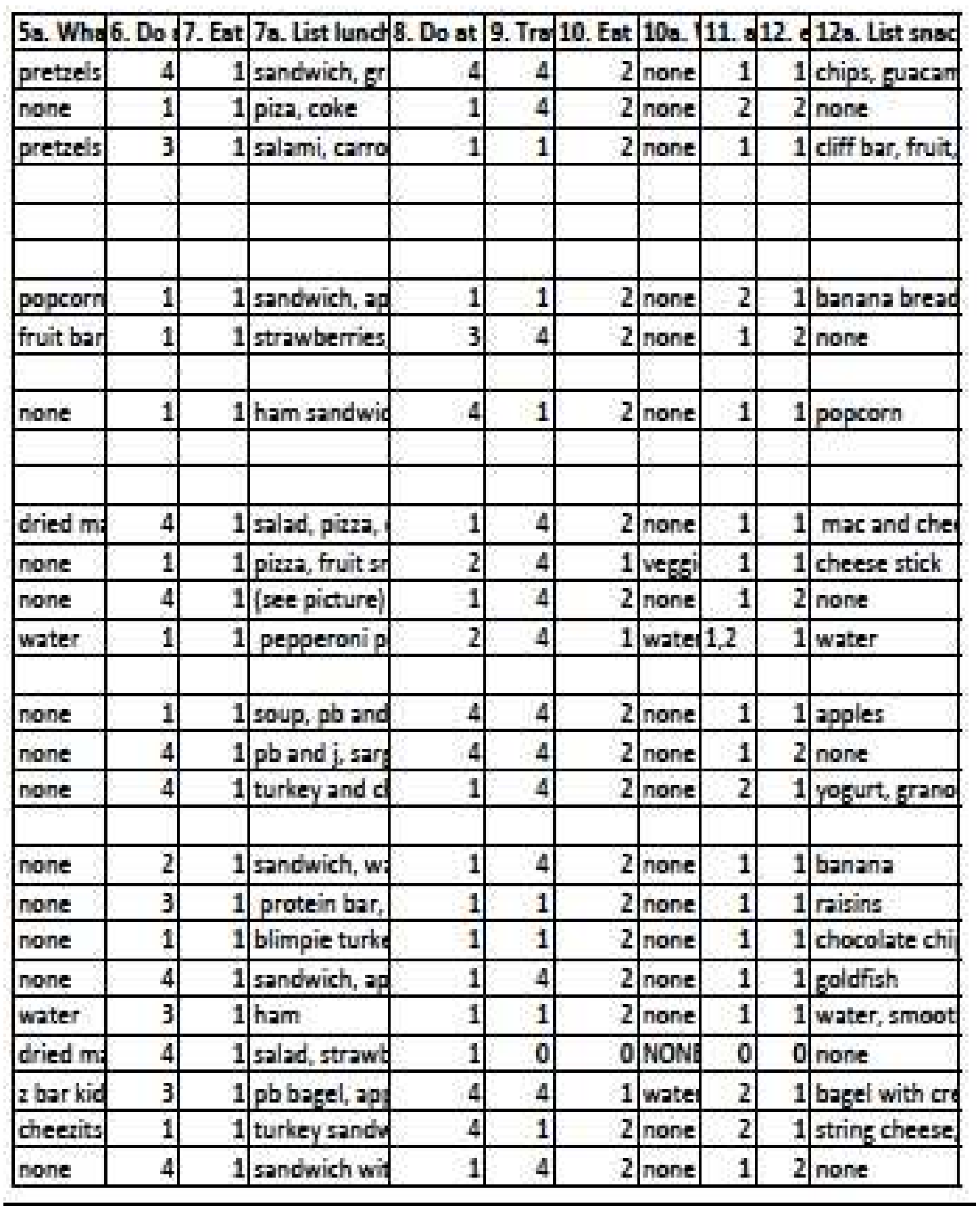




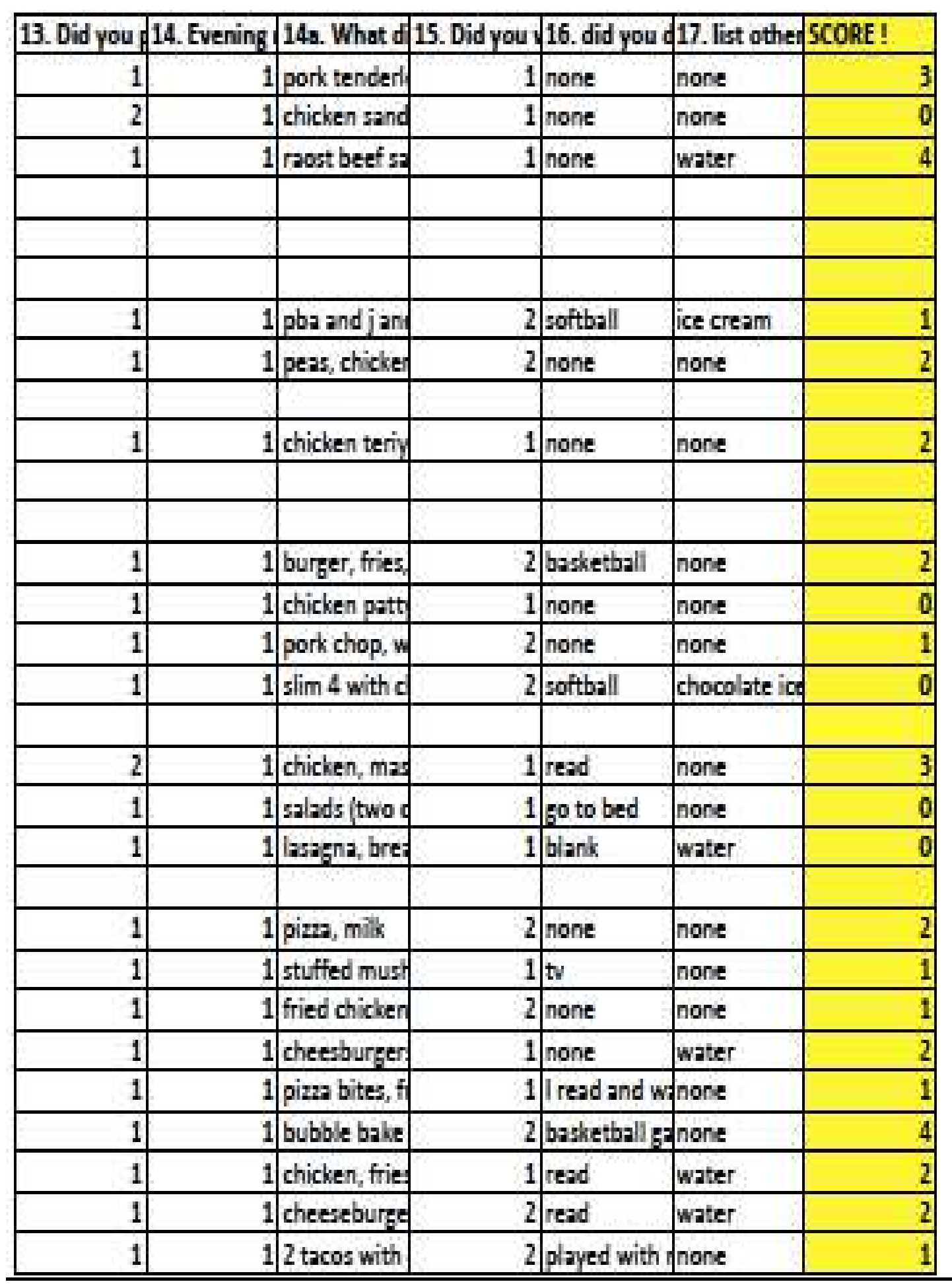




\begin{tabular}{|c|c|c|c|c|c|c|c|c|c|c|c|}
\hline & Surver ${ }^{3}$ & 1. Mever- & 10. Les: & JUICE & 2. Nev & 2. Les: & FROT & 3. $\mathrm{Ner}$ & $3 \mathrm{a.A}$ & FPUIT & 4. Never \\
\hline \multicolumn{12}{|l|}{$0026 \mathrm{~B}$} \\
\hline 00368 & & 0.706 & 1 & 0.79 & 3. & 1.5 & \begin{tabular}{|l|l|}
5 & 4.5 \\
\end{tabular} & 1 & 0.3 & 0.25 & 0.067 \\
\hline \multicolumn{12}{|l|}{00468} \\
\hline \multicolumn{12}{|l|}{00768} \\
\hline 00968 & & 0.5 & 0.5 & 0.25 & 2 & 1 & 2 & 0.5 & 0.5 & 0.25 & 0.067 \\
\hline 001168 & & 2 & 1.625 & 3.25 & 3 & 1 & 3 & 0.214 & 1 & \begin{tabular}{|l|}
0.214 \\
\end{tabular} & 0.214 \\
\hline 001468 & & 0.067 & $\overline{1}$ & 0.07 & 2 & 0.5 & 1 & 0.5 & 0.5 & 0.25 & 0.067 \\
\hline 001668 & $4 / 17 / 177$ & 0.067 & 0.5 & 0.03 & 4 & 0.5 & 2 & 0.214 & 1.5 & 0.321 & 0 \\
\hline \multicolumn{12}{|l|}{001968} \\
\hline 002068 & & 0.214 & 0.5 & 0.11 & 3 & 0.5 & \begin{tabular}{|l|l|}
5 & 15 \\
\end{tabular} & 0.214 & 0.5 & \begin{tabular}{|l|}
0.107 \\
\end{tabular} & 0.067 \\
\hline 002168 & & 3 & 0.5 & 15 & 3 & 0.5 & 1.5 & $\mid 0.214$ & 1.5 & 0.321 & 0.214 \\
\hline 002268 & & 0 & 0 & 0 & 2 & 1 & 2 & 0 & 0 & 0 & 0.214 \\
\hline 002468 & & 0.067 & 1 & 0.07 & 2 & 0.5 & 1 & $\mid$\begin{tabular}{|l|}
0.067 \\
\end{tabular} & 03 & \begin{tabular}{|l|}
0.017 \\
\end{tabular} & 0.214 \\
\hline 002568 & & 0.214 & 0.5 & 0.11 & 1 & 0.5 & \begin{tabular}{|l|l|}
5 & 0.5 \\
\end{tabular} & 0.214 & 2.5 & 0.535 & 0.214 \\
\hline \multicolumn{12}{|l|}{001568} \\
\hline \multicolumn{12}{|l|}{001268} \\
\hline \multicolumn{12}{|l|}{$0016 \mathrm{~F}$} \\
\hline $0028 \mathrm{~F}$ & & 0.214 & 1 & 0.21 & 3 & 0.5 & 1.5 & 1 & 1 & 1 & 0.067 \\
\hline $003 \mathrm{BF}$ & & 0.067 & 0.5 & 0.03 & 3 & 15 & 4.5 & 0.5 & 1 & 0.5 & 0.067 \\
\hline $0056 \mathrm{~F}$ & & 0.067 & 1 & 0.07 & 0.067 & & \begin{tabular}{|l|l|}
10.057 \\
\end{tabular} & 0.5 & 1 & 0.5 & 0.067 \\
\hline $0066 \mathrm{~F}$ & & 0.786 & 1 & 0.79 & 0.786 & & 10.786 & $\mid 0.067$ & 1 & \begin{tabular}{|l|}
0.067 \\
\end{tabular} & 0.786 \\
\hline \multicolumn{2}{|c|}{0010 6F (was entered : } & 0.5 & 0.5 & 0.25 & 0.706 & & 10.786 & 0.786 & 1 & 0.786 & 0 \\
\hline $00156 F$ & & 0.067 & $\overline{1}$ & 0.07 & 2 & 0.5 & 1) & 0 & 0 & 0 & 0.067 \\
\hline $00166 \mathrm{~F}$ & & 1 & 0.5 & 0.5 & 0.786 & $\overline{2}$ & \begin{tabular}{l|l|} 
& 1.572 \\
\end{tabular} & 0.214 & 1 & 0.214 & 0.214 \\
\hline $00200 \mathrm{~F}$ & & 0.5 & 1 & 0.5 & 3 & 15 & \begin{tabular}{|l|l|}
5 & 4.5 \\
\end{tabular} & 0.5 & 1 & 0.5 & 0.214 \\
\hline $00216 \mathrm{~F}$ & & 1 & 1 & 1 & 2 & 0.5 & 1 & 1 & 1 & 1 & 0 \\
\hline $00236 \mathrm{~F}$ & & 0 & $\overline{0}$ & 0 & 2 & 0.5 & 1 & 0 & (0) & 0 & 0.214 \\
\hline $00246 \mathrm{~F}$ & & & & & & & & & & & \\
\hline $00258 F$ & & & & & & & & & & & \\
\hline
\end{tabular}




\begin{tabular}{|c|c|c|c|c|c|c|c|c|c|c|c|}
\hline $4 a .5 \mathrm{~m}$ & LETTU & \multicolumn{2}{|c|}{ 5. New 5a. $1 \mathrm{H}$} & \multicolumn{2}{|c|}{ FAENCI 6. Never } & 6a. Le & \multicolumn{3}{|c|}{ OTHER 7.Neve 7 7 Less } & \multicolumn{2}{|c|}{\begin{tabular}{|l|l|} 
DRIE & B. Never: \\
\end{tabular}} \\
\hline 0.25 & 0.017 & 0.067 & 0.5 & 0.034 & 0.5 & 0.75 & 0.375 & 4 & 1.25 & 5 & 0214 \\
\hline & & & & & & & & & & & \\
\hline & & & & & & & & & & & \\
\hline 0.25 & 0.017 & 0.067 & 0.2 & 0.013 & 0 & 0.25 & 0] & 0.786 & 0.75 & 0.59 & 0.214 \\
\hline 0.5 & 0.107 & 0.214 & 0.5 & 0.107 & 0.067 & 0.25 & $\mid 0.017$ & 0.5 & 1.25 & 0.69 & 0.5 \\
\hline 0.5 & 0.034 & 0.067 & 0.5 & 0.034 & 0.214 & 0.75 & $\mid 0.161$ & \begin{tabular}{|l|}
0.214 \\
\end{tabular} & 0.75 & 0.16 & 0.214 \\
\hline 0 & 0 & o & 0 & o & 0.214 & 0.75 & 0.161 & 2 & 0.75 & 1.5 & 0.214 \\
\hline & & & & & & & & & & & \\
\hline 0.25 & 0.017 & 0.214 & 0.5 & 0.107 & 0.067 & 0.25 & \begin{tabular}{|l|}
0.017 \\
\end{tabular} & 1 & 0.75 & 0.75 & 0.067 \\
\hline 1 & 0.214 & 0.214 & 0.2 & 0.043 & 0.5 & 0.25 & 0.125 & 0.5 & 0.25 & 0.13 & 0214 \\
\hline 0.25 & 0.054 & 0.214 & 0.2 & 0.043 & 0 & 0 & 0 & \begin{tabular}{|l|}
0.786 \\
\end{tabular} & 0.75 & 0.59 & 0.214 \\
\hline 0.5 & 0.107 & 0.067 & 0.5 & 0.034 & 0 & 0 & 0 & 0.5 & 0.25 & 0.13 & 0.214 \\
\hline 0.25 & 0.054 & 0.067 & 0.2 & 0.013 & 0 & 0 & 0 & 1 & 0.75 & 0.75 & 0.214 \\
\hline & & & & & & & & & & & \\
\hline & & & & & & & & & & & \\
\hline & & & & & & & & & & & \\
\hline 0.5 & 0.034 & 可 & 0 & 0 & 0.067 & 0.5 & 0.034 & 0.214 & 0.75 & 0.16 & 0 \\
\hline 0.25 & 0.017 & 0.067 & 0.2 & 0.013 & 0.067 & 0.25 & $|0.017|$ & 1 & 0.75 & 0.75 & 0.214 \\
\hline 0.5 & 0.034 & 0.067 & 0.2 & 0.013 & 0.067 & 0.25 & 0.017 & 0.5 & 1.25 & 0.63 & 0.214 \\
\hline 0.5 & 0.393 & 0.067 & 0.2 & 0.043 & 0 & 0.25 & 0 & 0.5 & 0.75 & 0.38 & 0.786 \\
\hline 0] & 0 & 0,067 & 0,2 & 0.013 & 0 & 0 & 0] & \begin{tabular}{|l|l|}
0.786 \\
\end{tabular} & 0,75 & 0.59 & 0.067 \\
\hline 0.5 & 0.034 & 0.214 & 0.2 & 0.043 & 0.067 & 0.25 & $|0.017|$ & 1 & 0.75 & 0.75 & 0.214 \\
\hline 0.5 & 0.107 & 0.067 & 0.5 & 0.034 & 0.067 & 0.75 & 0.05 & 0.5 & 1.25 & 0.63 & 0.067 \\
\hline 0.5 & 0.107 & 0.214 & 0.5 & 0.107 & 0.5 & 1.2 & 0.6 & 0.786 & 1.25 & 0.98 & 0.5 \\
\hline a. & 0 & a. & $\overline{0}$ & 0 & 0 & 0 & o & 1 & 0.25 & 0.25 & 0.067 \\
\hline 0.25 & 0.054 & 0 & 0 & 0 & 0 & 0 & 0 & 0.5 & 0.25 & 0.13 & 0.214 \\
\hline & & & & & & & & & & & \\
\hline & & & & & & & & & & & \\
\hline
\end{tabular}




\begin{tabular}{|c|c|c|c|c|c|c|c|c|c|c|}
\hline Abou & OTHER V & Never= & $9 \mathrm{se}$ & TOMA & 10. Meen & 10a. Ábout & VEGETA & TotalEATS & Survey 4 & A. Ags \\
\hline & & & & & & & & & 100268 & 12 \\
\hline 15 & 0.321 & 0.067 & 1 & 0.067 & 0.766 & 2.25 & 1.7685 & 13.12 & $0036 \mathrm{~B}$ & 12 \\
\hline & & & & & & & & & $10046 \mathrm{~B}$ & \\
\hline & & & & & & & & & $0076 \mathrm{~B}$ & \\
\hline 15 & 0.321 & 0.067 & 0.2 & 0.017 & 0.5 & 0 & $\overline{0}$ & 3.46 & $0096 \mathrm{~B}$ & 12 \\
\hline 0.25 & 0.125 & 0.067 & 0.2 & 0.034 & 0.214 & 2.25 & 0.4815 & 7.96 & 1001168 & 12 \\
\hline 0.75 & 0.1605 & 0.214 & 0.5 & 0.107 & 0.786 & 0 & 0 & 1.97 & 100146B & 11 \\
\hline 0.25 & 0.0535 & 0 & 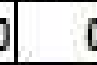 & 0 & 1 & $a$ & 1 & 5.07 & 001668 & 12 \\
\hline & & & & & & & & & $00196 \mathrm{~B}$ & \\
\hline 0.25 & 0.01675 & 0 & i & 0 & 0 & 0 & 0 & 2.62 & $00206 \mathrm{~B}$ & 12 \\
\hline 2.25 & 0.4815 & 0.067 & 70.2 & 0.034 & 0. & 0 & 0 & 4.34 & $00216 \mathrm{~B}$ & 12 \\
\hline 0.25 & 0.0535 & 0 & 0] & 0 & 0 & 0 & & 2.74 & $00226 \mathrm{~B}$ & 12 \\
\hline 0.75 & 0.1605 & 0 & 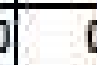 & 0 & 0.214 & 0 & 0 & 3.77 & 002468 & 12 \\
\hline 0.25 & 0.0535 & $\overline{0}$ & 7 & 0 & 0.214 & 0.3 & 0.0642 & 151 & 002568 & 11 \\
\hline & & & & & & & & & 1001568 & \\
\hline & & & & & & & & & 1001268 & \\
\hline & & & & & & & & & $10018 \mathrm{FF}$ & \\
\hline 0 & 0 & 0.214 & 0.78 & 0.16 & 0.5 & -1 & 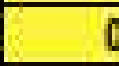 & 2.69 & $0026 \mathrm{~F}$ & 12 \\
\hline 0.75 & 0.1605 & 0.067 & 0.9 & 0.03 & 0.214 & 1.6 & 0.3424 & 6.33 & $0036 \mathrm{~F}$ & 11 \\
\hline 0.75 & 0.1605 & 0.067 & 0.9 & 0.03. & 0.5 & 0 & 0 & 1.45 & $0056 \mathrm{~F}$ & 12 \\
\hline 1.5 & 1.179 & l & 0.2 & 1 & 0.5 & 0.5 & 0.25 & 3.06 & $0066 \mathrm{~F}$ & 12 \\
\hline 0.25 & 0.01675 & 0 & j] & & 0.765 & 7 & 0.786 & 2.98 & $100106 \mathrm{~F}$ & 12 \\
\hline 0.25 & 0.0535 & 0 & ] & 1 & 0 & ] & I & & $00156 \mathrm{~F}$ & 12 \\
\hline 2.25 & 0.15075 & 0.067 & 70.5 & 0.03 & \begin{tabular}{|l|}
0.067 \\
\end{tabular} & 225 & 0.150 & 2.94 & 1001686 & $\overline{12}$ \\
\hline 0.75 & 0.375 & 0.067 & & 0.06 & 0.5 & 0 & 6 & 7.24. & 1002067 & 12 \\
\hline 0.25 & 0.01675 & 0.067 & 0.2 & 0.03 & \begin{tabular}{|l|}
0.067 \\
\end{tabular} & 1 & 0.067 & 2.37 & $00216 \mathrm{~F}$ & 12 \\
\hline 0.75 & 0.1605 & 0 & if & I & 0.214 & 0 & 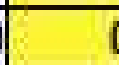 & 134 & $100236 \mathrm{BF}$ & \\
\hline & & & & & & & & & $100246 \mathrm{FF}$ & 12 \\
\hline & & & & & & & & & $00258 \mathrm{~F}$ & 17 \\
\hline
\end{tabular}




\begin{tabular}{|c|c|c|c|c|c|c|c|c|c|}
\hline $2, \mathrm{M}=1 / \mathrm{F}=2$ & 5. yes: $1, \mathrm{no}$ & $5 a, 1-2=1,3$ & 6 . yes=1, no: & 6s. Colcium & 7. Let 7 & & FRUT: & 7c. Less: & 7d. Le \\
\hline 1 & 0 & 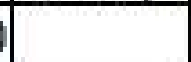 & 0 & & 1 & 4 & 0.714 & 0 & 1 \\
\hline 1 & 1 & 3 & 0 & & 4 & 1 & 0.714 & 1 & 4 \\
\hline & & & & & & & & & \\
\hline & & & & & & & & & \\
\hline 2 & 0 & 0 & 0 & 0 & 2 & 4 & 0.857 & 0 & 2 \\
\hline 1 & 1 & 2 & 0 & & 3 & 4 & 1 & 0 & 1 \\
\hline 2 & 0 & 0 & 0 & 0 & 1 & 5 & 0.857 & 0 & 3 \\
\hline 2 & 1 & 3 & 1 & 6 & 2 & 0 & 0.286 & 0 & 0 \\
\hline & & & & & & 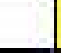 & & 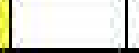 & \\
\hline 1 & 1 & 2 & 0 & 0 & 0 & 5 & 0.714 & 0 & 1 \\
\hline 2 & & L & 1 & t & 0 & 3 & 0.429 & 0 & 3 \\
\hline 1 & 0 & 0 & 0 & 0 & 0 & 5 & 0.714 & 0 & 0 \\
\hline 2 & 1 & 2 & 0 & & 0 & 4 & 0.571 & 0 & 0 \\
\hline 1 & 0 & 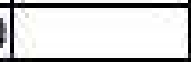 & 0 & & 2 & 4 & 0.857 & 0 & 0 \\
\hline & & & & & & & & & \\
\hline & & & & & & & & & \\
\hline & & & & & & & & & \\
\hline 1 & 0 & 0 & 0 & 0 & 0 & 3 & 0.429 & 0 & 2 \\
\hline 2 & 0 & t & 0 & 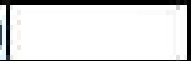 & 1 & 5 & 0.857 & 0 & 2 \\
\hline 2 & 0 & & 0 & & 0 & 4 & 0.571 & 0 & 4 \\
\hline 1 & 0 & 1 & 0 & 4 & 2 & 2 & 0.571 & 1 & 1 \\
\hline 1 & 0 & 0 & 0 & 0 & 0 & 4 & 0.571 & 0 & 4 \\
\hline 1 & 1 & 3 & 0 & & 0 & 5 & 0.714 & 0 & 0 \\
\hline 2 & 0 & 0 & 0 & 1 & 4 & 4 & 1.143 & 1 & 2 \\
\hline 2 & 1 & 3 & 0 & 0 & 3 & 5 & 1.143 & 0 & 2 \\
\hline 1 & 1 & 3 & 1 & 3 & 4 & 4 & 1.143 & 1 & 3 \\
\hline 2 & & & & & 0 & 5 & 0.714 & 0 & 4 \\
\hline 2 & 0 & 0 & 0 & 3 & 4 & 3 & 1 & 0 & 2 \\
\hline 1 & 1 & 3 & 1 & 4 & 4 & 4 & 1.143 & 0 & 3 \\
\hline
\end{tabular}




\begin{tabular}{|c|c|c|c|c|c|c|c|c|c|c|c|c|c|c|}
\hline 77.7 & & Veget & 7e. Less that & POTA & 4.4 & -4 & FIBER & $\mathrm{Be}$ & BEAN: & "Tot: & chet & List of & 1. Never & 30. \\
\hline 1 & 4 & 0.86 & 1 & 0.143 & 1 & 3 & 0.57 & 2 & 0.29 & 2 & 1 & 1 & 3 & 1 \\
\hline 2 & 4 & \begin{tabular}{|l|l}
1.57 \\
\end{tabular} & 1 & 0.143 & 1 & 1 & 0.29 & 2 & 0.29 & 2.71 & & & 0 & 0 \\
\hline & & & & & & & & & & & & & & \\
\hline & & & & & & & & & & & & & & \\
\hline 0 & 2 & 0.57 & 1 & 0.143 & 2 & 0 & 0.29 & 0 & 0 & 1.57 & & 4 & 4 & 0 \\
\hline 0 & 3 & 0.57 & 3 & 0.429 & 0 & 0 & 0 & 0 & 0 & 2 & & 6. & 1 & 0 \\
\hline 1 & 3) & 1 & 1 & 0.143 & 0 & 3 & 0.43 & 3 & 0.43 & 2.43 & & & 5 & 1 \\
\hline 2 & 2 & 0.57 & 0 & 0 & 3 & 2 & 0.71 & 2 & 0.29 & 1.14 & 6 & 4 & 1 & 0 \\
\hline & & & & & & & & & & & & & & \\
\hline 0 & 5 & 0.86 & 2 & 0.266 & 0 & 0 & 0] & 1 & 0.14 & 2 & 3 & 3 & 3 & 0 \\
\hline 1 & 0 & 0.57 & 1 & 0.143 & 3 & 3 & 0.86 & 0 & 0 & 1.14 & 7 & 2) & 4 & 1 \\
\hline 0 & 5 & 0.71 & 3 & 0.429 & 0 & 3 & 0.43 & 0 & 0 & 1.85 & $\theta$ & 4 & 3 & 0 \\
\hline 0 & 3 & 0.43 & 2 & 0.266 & 2 & 2 & 0.57 & 0 & 0 & 1.29 & & 5 |crsipix & 3 & 0 \\
\hline 0 & 4 & 0.57 & 0 & 0 & 0 & 2 & 0.29 & 0 & 0 & 1.43 & - & 5 & 0 & 0 \\
\hline & & & & & & & & & & & & & & \\
\hline & & & & & & & & & & & & & & \\
\hline & & & & & & & & & & & & & & \\
\hline 1 & 2 & 0.71 & 0 & 0 & 0 & 0 & 0 & $\sqrt{2}$ & 0.29 & 1.43 & & 5 cocol & 3 & 0 \\
\hline 1 & 4 & 1 & 0 & 0 & 0 & 0 & 0 & 0 & 0 & 1.86 & & 6 fiber of & 4 & 4 \\
\hline 0 & 4 & 1.14 & 1 & 0.143 & 4 & 4 & 1.14 & 1 & 0.14 & 2 & & (5rano & 1 & 0 \\
\hline 0 & 0 & 0.29 & 2 & 0.286 & 0. & 2 & 0.29 & 0 & 0 & 1.14 & 2 & 2 & 5 & 0 \\
\hline 0 & 1 & 0.71 & 0 & 0 & 0 & 0 & 0 & 0 & 0 & 1.29 & & 0 & 0 & 1 \\
\hline 1 & 3 & 0.57 & 1 & 0.143 & 1 & 4 & 0.71 & 2 & 0.29 & 1.71 & 3 & 1 & 3 & 1 \\
\hline 2 & 2 & 1 & 3 & 0.429 & 2 & 0 & 0.29 & 0 & 0 & 2.57 & 2 & 2 & 2 & 3. \\
\hline 1 & 3 & 0.86 & 1 & 0.143 & 1 & 2 & 0.43 & 1 & 0.14 & 229 & & 6 Grang & 3 & 3 \\
\hline 0 & 3 & 1 & 0 & 0 & 0 & 0 & 0 & 0 & 0 & 2.14 & 2 & 2 & 4 & 2 \\
\hline 1 & 4 & 1,29 & 1 & 0.143 & 0 & 0 & 0 & 2 & 0.29 & 243 & & 6 | Never & 0 & 0 \\
\hline 0 & 4 & 0.86 & 2 & 0.266 & 2 & 0 & 0.29 & 0 & 0 & 2.14 & 1 & 1 & 4 & 0 \\
\hline 0 & 4 & 1 & 2 & 0.286 & 2 & 3 & 0.71 & 2 & 0.29 & 271 & 2 & 2 & 3 & 0 \\
\hline
\end{tabular}




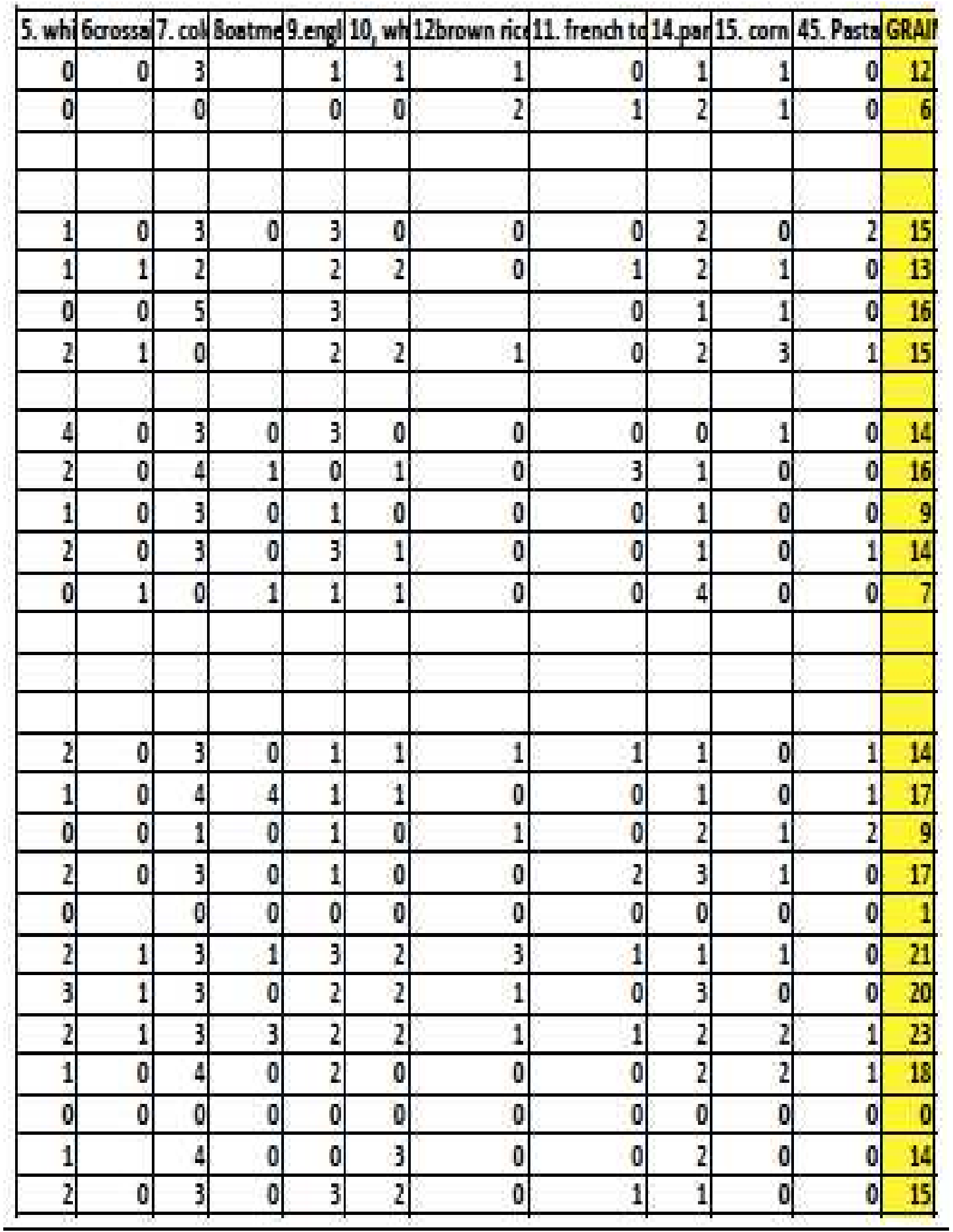




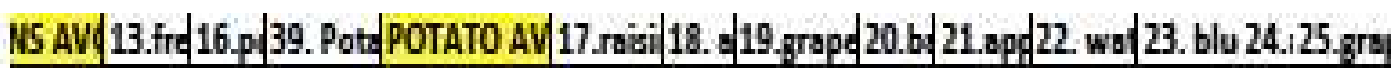

\begin{tabular}{|c|c|c|c|c|c|c|c|c|c|c|c|c|c|c|c|}
\hline 0.4 & 2 & 1 & & 3 & 0.1 & 1 & 3 & & 2 & 3 & 1 & 3 & & 1 & \\
\hline 0.2 & 1 & 1 & 0 & 2 & 0.07 & 0 & 0 & & 4 & 2 & 1 & 4 & 4 & 2 & \\
\hline & & & & & & & & & & & & & & & \\
\hline & & & & & & & & & & & & & & & \\
\hline 0.5 & 0 & 1 & 0 & 1) & 0.03 & 0 & 3 & & 2 & 3 & 1 & 2 & 2 & 3) & 1 \\
\hline 0.43 & 2 & 1 & 1 & 4 & 0.13 & 1 & 1 & & 3 & 3) & 1 & 1 & 1 & 3] & \\
\hline 0.53 & & 2 & 0 & 2 & 0.07 & 0 & 2 & & 1 & 4 & 1 & 2 & 2 & 1 & 0 \\
\hline 0.5 & 2 & 2 & 1 & 5 & 0.17 & 1 & 3 & 2 & 0 & 3 & 2 & & 0 & 3) & \\
\hline & & & & & & & & & & & & & & & \\
\hline 0.47 & 2 & 2 & 0 & 4 & 0.13 & 1 & 0 & & 3 & 3 & 0 & 0 & 0. & 이 & 0 \\
\hline 0.53 & 2 & 1 & 0 & 3] & 0.1 & 0 & 0 & & 1 & 1 & 0 & 0 & 0 & 0 & 0 \\
\hline 0.3 & 2 & 1 & 0 & 3 & 0.1 & 1 & 3 & & 1 & 1 & 1 & 0 & 0 & 0 & 0 \\
\hline 0.47 & 2 & 1 & 1 & 4 & 0.13 & 0 & 3 & & 3 & 3 & 2 & 4 & 4 & 1 & 0 \\
\hline 0.23 & 2 & 1 & 0 & 3 & 0.1 & 0 & 0 & & 1 & 3 & 1 & i) & 0 & 2 & \\
\hline & & & & & & & & & . & & . & & & & \\
\hline & & & & & & & & & & & & & & & \\
\hline & & & & & & & & & & & 1 & & & & \\
\hline 0.47 & 1 & 0 & 0 & 1 & 0.03 & 0 & 0 & & 3 & 2 & 1) & 1 & 1 . & 1 & 1 \\
\hline 0.57 & 1 & 1 & 0 & 2 & 0.07 & 0 & 0 & & 3 & 3 & 2 & & 3 & 1 & 0 \\
\hline 0.3 & 0 & 1 & 1 & 2 & 0.07 & 0 & 0 & & 3 & 3 & 0 & 3 & 3 & 0 & 0 \\
\hline 0.57 & 3 & 0 & 0 & 3 & 0.1 & 1 & 0 & & 2 & 3 & 2 & 1 & 1 . & 2 & 0 \\
\hline 0.03 & 0 & 0 & 0 & 0) & 0 & 1 & 0 & & 2 & 2 & 0 & 2 & 2 & 이 & 0 \\
\hline 0.7 & 2 & 2 & 0 & 4 & 0.13 & 1 & 3 & & 2 & 3 & 1 & 1 & 2 & 1 & 1 \\
\hline 0.67 & 1 & 1 & 0 & 2 & 0.07 & 0 & 0 & & 0 & 3 & 0 & 1 & 1 & 1 & 0 \\
\hline 0.77 & 2 & 2 & 0 & 4 & 0.13 & 3 & 3 & & 4 & 3) & 2 & 3 & \begin{tabular}{l|l}
3 &
\end{tabular} & 3 & 1 \\
\hline 0.6 & 1 & 0 & 0 & 1) & 0.03 & 4 & 4 & & 2 & 2 & 0 & 2 & 2 & 1 & 0 \\
\hline 0 & 1 & 0 & & 1 & 0.03 & 0 & 0 & & 4 & 4 & 0 & 4 & 4 & 0 & 0 \\
\hline 0.47 & 2 & 2 & 0 & 4 & 0.13 & 0 & 4 & & 1 & 0 & 1 & 0 & 0 & 3] & \\
\hline 0.5 & 1 & 1 & 0 & 2 & 0.07 & 0 & 2 & & 1 & 1 & 1 & 1 & 2 & 4 & \\
\hline
\end{tabular}


26. Pin27. Con 28. Stri29. Ora30. Orar 31. PeaFBUT / per d 33. Bi46. G 52. PaLEU perda4 4. Salsa

\begin{tabular}{|r|r|r|r|r|r|r|r|r|r|r|r|r|r|}
\hline 0 & 1 & 3 & 1 & 3 & 0 & 25 & 0.83 & 2 & 3 & 1 & 6 & 0.2 & 2 \\
\hline 3 & 4 & 4 & 0 & 0 & 4 & 30 & 1 & 3 & 0 & 2 & 5 & 0.157 & 1 \\
\hline & & & & & & & & & & & & & \\
\hline & & & & & & & & & & & & & \\
\hline 0 & 2 & 4 & 2 & 3 & 1 & 30 & 1 & 0 & 1 & 2 & 3 & 0.1 & 2 \\
\hline 2 & 0 & 3 & 2 & 1 & 0 & 23 & 0.77 & 0 & 2 & 1 & 3 & 0.1 & 2 \\
\hline 2 & 1 & 3 & 1 & 3 & 2 & 26 & 0.87 & 3 & 1 & 2 & 6 & 0.2 & \\
\hline 0 & 0 & 3 & 1 & 0 & 0 & 13 & 0.6 & 3 & 0 & 0 & 3 & 0.1 & 3 \\
\hline & & & & & & & & & & & & & \\
\hline 0 & 0 & 3 & 0 & 2 & 0 & 13 & 0.43 & 1 & 2 & 1 & 4 & 0.133 & 0 \\
\hline 1 & 0 & 4 & 0 & 1 & 1 & 9 & 0.3 & 0 & 0 & 0 & 0 & 0 & 1 \\
\hline 1 & 1 & 3 & 0 & 3 & 1 & 19 & 0.63 & 0 & 3 & 1 & 4 & 0.133 & 0 \\
\hline 1 & 0 & 4 & 1 & 4 & 1 & 20 & 0.97 & 1 & 4 & 1 & 6 & 0.2 & 1 \\
\hline 1 & 0 & 3 & 2 & 1 & 1 & 19 & 0.63 & 0 & 1 & 0 & 1 & 0.033 & 1 \\
\hline & & & & & & & & & & & & & \\
\hline & & & & & & & & & & & & & \\
\hline & & & & & & & & & & & & & \\
\hline 1 & 1 & 1 & 2 & 3 & 1 & 20 & 0.67 & 1 & 1 & 1 & 3 & 0.1 & 1 \\
\hline 1 & 0 & 4 & 1 & 0 & 1 & 22 & 0.73 & 0 & 0 & 2 & 2 & 0.067 & 0 \\
\hline 3 & 0 & 4 & 0 & 3 & 0 & 21 & 0.7 & 0 & 1 & 0 & 1 & 0.033 & 1 \\
\hline 2 & 0 & 1 & 3 & 1 & 0 & 20 & 0.67 & 0 & 0 & 0 & 0 & 0 & 1 \\
\hline 1 & 0 & 2 & 2 & 3 & 0 & 16 & 0.53 & 0 & 1 & 1 & 2 & 0.067 & 1 \\
\hline 1 & 1 & 3 & 1 & 3 & 1 & 26 & 0.87 & 2 & 2 & 3 & 7 & 0.233 & 1 \\
\hline 0 & 0 & 1 & 4 & 3 & 1 & 15 & 0.5 & 0 & 1 & 0 & 1 & 0.033 & 3 \\
\hline 2 & 2 & 4 & 3 & 3 & 3 & 42 & 1.4 & 2 & 1 & 1 & 4 & 0.133 & 2 \\
\hline 1 & 0 & 4 & 4 & 1 & 0 & 27 & 0.9 & 0 & 0 & 0 & 0 & 0 & 1 \\
\hline 0 & 0 & & & & & 14 & 0.47 & & 1 & 0 & 1 & 0.033 & 2 \\
\hline 0 & 1 & 0 & 3 & 0 & 0 & 13 & 0.43 & 0 & 2 & 0 & 2 & 0.067 & 3 \\
\hline 1 & 1 & 2 & 0 & 0 & 1 & 17 & 0.57 & 1 & 3 & 2 & 6 & 0.2 & 1 \\
\hline
\end{tabular}


32. Tom 33. Beana 34. Tome 35. Broo 36. Mixe 37. Yam 38. Cel40. Vi14. Coul 42. Cab 43. Zu 44. Le

\begin{tabular}{|r|r|r|r|r|r|r|r|r|r|r|r|}
\hline 0 & 2 & 4 & 3 & 2 & 1 & 1 & 0 & 1 & 0 & 2 & 1 \\
\hline 4 & 3 & 1 & 3 & 3 & 0 & 4 & 0 & 0 & 0 & 3 & 4 \\
\hline & & & & & & & & & & & \\
\hline 1 & & & & & & & & & & & \\
\hline 1 & 0 & 0 & 2 & 2 & 2 & 2 & 0 & 2 & 0 & 0 & 2 \\
\hline 3 & 3 & 0 & 2 & 2 & 0 & 2 & 0 & 0 & 0 & 1 & 3 \\
\hline 3 & 3 & 0 & 0 & 0 & 1 & 0 & 0 & 0 & 0 & 1 & 1 \\
\hline & & & & & & & & & & & \\
\hline 0 & 1 & 0 & 3 & 3 & 0 & 0 & 0 & 0 & 0 & 0 & 2 \\
\hline 0 & 0 & 0 & 0 & 0 & 1 & 0 & 0 & 0 & 0 & 0 & 3 \\
\hline 0 & 0 & 0 & 0 & 0 & 0 & 0 & 0 & 0 & 0 & 0 & 0 \\
\hline 0 & 1 & 0 & 3 & 2 & 0 & 0 & 0 & 2 & 1 & 0 & 1 \\
\hline 1 & 0 & 0 & 2 & 0 & 0 & 1 & 0 & 0 & 0 & 0 & 2 \\
\hline & & & & & & & & & & & \\
\hline & & & & & & & & & & & \\
\hline & & & & & & & & & & & \\
\hline 3 & 1 & 0 & 0 & 0 & 0 & 0 & 0 & 0 & 0 & 0 & 4 \\
\hline 0 & 0 & 0 & 3 & 1 & 0 & 0 & 0 & 1 & 0 & 0 & 2 \\
\hline 1 & 0 & 0 & 3 & 3 & 1 & 0 & 1 & 1 & 1 & 0 & 3 \\
\hline 1 & 0 & 0 & 1 & 1 & 0 & 1 & 0 & 1 & 0 & 0 & 1 \\
\hline 2 & 0 & 0 & 3 & 4 & 0 & 2 & 0 & 1 & 2 & 0 & 3 \\
\hline 1 & 2 & & & & 1 & 1 & 0 & 1 & 1 & 1 & 1 \\
\hline 1 & 0 & 0 & 1 & 1 & 0 & 0 & 1 & 0 & 0 & 0 & 3 \\
\hline 3 & 2 & 0 & 2 & 3 & 1 & 1 & 0 & 1 & 1 & 1 & 2 \\
\hline 0 & 0 & 0 & 0 & & 0 & 0 & 2 & 0 & 0 & 0 & 2 \\
\hline & & & & & & & 0 & & & 0 & 4 \\
\hline 0 & 0 & 0 & 3 & 1 & 2 & 0 & 0 & 0 & 0 & 3 & 3 \\
\hline 3 & 1 & 0 & 2 & 1 & 0 & 0 & 0 & 0 & 0 & 1 & 3 \\
\hline & & & & & & & & & \\
\hline
\end{tabular}


47. Co 48. collas 49. Com 50. Col51. Oha 53. Gret 54. ComotVEG

PER O| 'TOTALI

\begin{tabular}{|r|r|r|r|r|r|r|r|r|r|}
\hline 2 & 0 & 0 & 0 & 0 & 1 & 3 & 25 & 0.833 & 1.97 \\
\hline 2 & 2 & 0 & 1 & 0 & 4 & 0 & 35 & 1.167 & 2.4 \\
\hline & & & & & & & & & \\
\hline & & & & & & & & & \\
\hline 1 & 0 & 2 & 0 & 0 & 2 & 2 & 22 & 0.733 & 1.27 \\
\hline 2 & 0 & 1 & 0 & 0 & 3 & 2 & 17 & 0.567 & 1.57 \\
\hline 1 & 1 & 2 & 0 & 0 & 4 & 3 & 27 & 0.9 & 2.03 \\
\hline 3 & 0 & 0 & 0 & 0 & 1 & 0 & 16 & 0.533 & 1.4 \\
\hline & & & & & & & & & \\
\hline 2 & 0 & 2 & 0 & 0 & 2 & 1 & 16 & 0.533 & 1.23 \\
\hline 1 & 0 & 1 & 0 & 0 & 0 & 1 & 3 & 0.267 & 0.67 \\
\hline 2 & 0 & 3 & 0 & 0 & 0 & 0 & 5 & 0.167 & 1.03 \\
\hline 1 & 1 & 0 & 0 & 0 & 0 & 0 & 13 & 0.433 & 1.73 \\
\hline 1 & 0 & 0 & 0 & 0 & 2 & 3 & 13 & 0.433 & 1.2 \\
\hline & & & & & & & & & \\
\hline & & & & & & & & & \\
\hline & & & & & & & & & \\
\hline 1 & 0 & 0 & 0 & 0 & 1 & 2 & 13 & 0.433 & 1.23 \\
\hline 1 & 0 & 1 & 0 & 0 & 0 & 3 & 12 & 0.4 & 1.27 \\
\hline 1 & 3 & 0 & 1 & 0 & 3 & 1 & 24 & 0.8 & 1.6 \\
\hline 1 & 0 & 0 & 1 & 0 & 0 & 3 & 12 & 0.4 & 1.17 \\
\hline 0 & 4 & 1 & 0 & 0 & 1 & 2 & 26 & 0.867 & 1.47 \\
\hline 1 & 1 & 1 & 0 & 0 & 2 & 4 & 19 & 0.633 & 1.87 \\
\hline 1 & 0 & 1 & 0 & 0 & 3 & 1 & 16 & 0.533 & 1.13 \\
\hline 1 & 1 & 2 & 1 & 0 & 2 & 3 & 29 & 0.967 & 2.63 \\
\hline 1 & 0 & 0 & 0 & 0 & 0 & 4 & 10 & 0.333 & 1.27 \\
\hline 0 & 3 & 1 & 0 & 0 & 3 & 2 & 15 & 0.5 & 1.03 \\
\hline 2 & 0 & 0 & 0 & 0 & 3 & 1 & 21 & 0.7 & 1.33 \\
\hline 2 & 0 & 0 & 0 & 0 & 0 & 4 & 18 & 0.6 & 1.43 \\
\hline & & & & & & & \\
\hline 1
\end{tabular}




\section{Post DATA}

\begin{tabular}{|c|c|c|c|c|c|c|c|c|c|c|c|c|c|c|c|}
\hline th & Subj Stud & $\overline{A G E}$ & Gen & Height $\mathrm{Pr}$ & $m$ & Weight B & BMI & z score & CDC Percen & Pre Systo & Pre Diastd & Base & Num & Num & Tota \\
\hline WB & $0026 \mathrm{~B}$ & 12 & 1 & 166 & 1.66 & 54.00 & 19.629 & 0.5 & 50 th -75 th & 103 & 56 & 1 & 1 & 1 & 2 \\
\hline TB & $0036 \mathrm{~B}$ & 12 & 1 & 153.5 & 1.535 & 44.09 & 18.712 & 0.5 & 50 th -75 th & 108 & 81 & 1 & 1 & 0 & 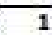 \\
\hline WD & $0048 B$ & 12 & 1 & 155 & 1.55 & 38.64 & 16.083 & -1 & 10 th- -25 th & 107 & 51 & 1 & 1 & 0 & 1 \\
\hline DF & $0076 \mathrm{~B}$ & 12 & 1 & 160 & 1.6 & 40.9 & 15.977 & -1 & 10th-25th & 85 & 64 & 1 & 0 & 0 & 0 \\
\hline AK & $0096 \mathrm{~B}$ & 12 & 2 & 153.5 & 1.535 & 55.8 & 23.724 & 1.5 & 90th-85th & 119 & 76 & 1 & 0 & 1 & 1 \\
\hline J & $00116 \mathrm{~B}$ & 12 & 1 & 150 & 1.5 & 45.91 & 20.404 & 0.5 & 50 th -75 th & 89 & 56 & 1 & 1 & 1 & 4 \\
\hline MP & $00146 \mathrm{~B}$ & 11 & 2 & 171 & 1.71 & 76.36 & 26.114 & 2 & $>25$ th & 113 & 55 & 1 & 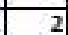 & 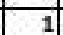 & - \\
\hline$E R$ & DO168B & 12 & 2 & 59.25 & .5925 & 50.45 & 19.883 & 0.5 & 50 th -75 th & 115 & 65 & 4 & 1 & 0 & 1 \\
\hline CS & 001968 & 11 & 2 & 156.75 & 1.5675 & 44.54 & 18.127 & 0 & 50th-75th & 85 & 53 & 1 & 1 & 0 & 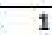 \\
\hline JS & $00206 \mathrm{~B}$ & 12 & 1 & 152 & 1.52 & 60.68 & 26.264 & 2 & $>95$ th & 116 & 87 & 1 & 2 & 0 & 2 \\
\hline SS & $00216 B$ & 12 & 2 & 162 & 1.62 & 44.32 & 16.888 & -0.5 & 25 th-50th & 100 & 88 & 1 & 0 & 0 & 0 \\
\hline$\overline{D T}$ & $00226 \mathrm{~B}$ & 12 & 1 & 155 & 1.55 & 39.55 & 16.462 & -0.5 & 10th & 86 & 55 & 1 & 1 & 0 & 1 \\
\hline BW & $00248 B$ & 12 & 2 & 148.5 & 1.485 & 38.86 & 17.622 & 1 & 25 th-50th & 104 & 63 & 1 & 1 & 0 & 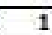 \\
\hline GZ & $00256 \mathrm{~B}$ & 12 & 1 & 142.4 & 1.425 & 33.18 & 16.34 & 0 & 10th-25th & 101 & 68 & & & & \\
\hline GR & 00156B & 11 & 2 & 158.5 & 1.585 & 52.73 & 20.989 & -0.5 & 85th & 108 & 64 & 1 & 3.5 & 2 & 5.5 \\
\hline GL & $00126 \mathrm{~B}$ & 11 & 2 & 146 & 1.46 & 36.59 & \begin{tabular}{|l|}
17.166 \\
\end{tabular} & 1 & 25 th-50th & 90 & 79 & 1 & 1.5 & 0 & 1.5 \\
\hline KC & $0016 \mathrm{~F}$ & 12 & 2 & 154 & 1.54 & 51.545 & 21.734 & 1 & 75th-85th & 102 & 60 & 1 & 0 & 0.5 & 0.5 \\
\hline TD & $0026 \mathrm{~F}$ & 12 & 1 & 154.87 & 1.5487 & 63 & 26.267 & 2 & $>85$ th & 118 & 71 & 1 & 1 & 0 & 1 \\
\hline$E D$ & $0036 \mathrm{~F}$ & 11 & 2 & 146.25 & 1.4625 & 38.545 & 18.021 & 0.5 & 50 th- 75 th & 106 & 82 & 1 & 7 & 이 & - \\
\hline MG & $0056 \mathrm{~F}$ & 12 & 2 & 154.5 & 1.545 & 40 & 16.757 & -0.5 & 25 th-50th & 97 & 88 & 1 & 2.68 & 0 & 27 \\
\hline$\overline{\mathrm{AH}}$ & $0068 \mathrm{~F}$ & 12 & 1 & 142 & 1.42 & 44.91 & 22.272 & 1 & 85th-90th & 108 & 58 & 1 & 0 & 1.5 & 1.5 \\
\hline AK & $00106 \mathrm{~F}$ & 12 & 1 & 154.25 & 1.5425 & 52.04 & 21.872 & 1 & 85th-90th & 102 & 87 & 1 & & & \\
\hline JM & $00158 \mathrm{~F}$ & 12 & 1 & 183.5 & 1.635 & 49 & 18.33 & of & 50 th- & 95 & 63 & 1 & 1 & 이 & 1 \\
\hline WM & $00166 \mathrm{~F}$ & 12 & 1 & 180.25 & 1.6025 & 48.18 & 18.762 & of: & 50 th -75 th & 98 & 70 & 1 & 4 & 0 & - \\
\hline$\overline{A O}$ & $00206 \mathrm{~F}$ & 12 & 2 & 162 & 1.62 & 48 & 17.528 & -0.5 & 25 th-50th & 101 & 53 & t & 0.5 & 0 & 0.5 \\
\hline WP & $00216 \mathrm{~F}$ & 12 & 1 & 145 & 1.45 & 41.91 & 19.833 & 0.5 & 50 th-75th & 81 & 73 & 1 & 2 & 0 & 7 \\
\hline$\overline{M R}$ & $00236 \mathrm{~F}$ & 12 & 2 & 152.5 & 1.525 & 36.36 & 15.635 & -1 & 10th-25th & 90 & 64 & 1 & 1 & 0 & 1 \\
\hline$\overline{C S}$ & $00246 \mathrm{~F}$ & 12 & 2 & 160 & 1.6 & 40 & 15.625 & -1 & 10th-25th & 100 & 85 & 1 & 1 & 0 & 1 \\
\hline CW & $00256 \mathrm{~F}$ & 11 & 1 & 160 & 1.8 & 54.91 & 21.449 & 1.5 & 85th & 103 & 85 & 1 & 0 & 0 & 0 \\
\hline
\end{tabular}

\begin{tabular}{|c|c|c|c|c|c|c|c|c|c|c|c|c|c|c|c|}
\hline Types of servings & Comments & $\%$ Cons & Est \% & $\mathrm{dD}$ & Baseli & Nurn & Numb & Tot: & Types of servings & $\%$ consum & est con & risum & Baselin & $N$ & Nun \\
\hline Erapes, carrots & & 100 & 2 & $0026 \mathrm{~B}$ & 2 & 1 & 1 & 2 & applesauce, carrots & 100 & 2 & & 3 & 2 & 0 \\
\hline strawberries & & 100 & 1 & $0036 \mathrm{~B}$ & 2 & 1 & 1.25 & 2.3 & carrots, salad (1.5 cy & 100 & 2.25 & & 3 & 0 & 0.3 \\
\hline appleasuce & & 100 & 1 & $0046 \mathrm{~B}$ & 2 & 1 & 0.5 & 1.5 & apples, carrots & 100 & 1.5 & & 3 & 1 & 0 \\
\hline sweet peppers & & & & $0096 \mathrm{~B}$ & 2 & 1 & 1 & 2 & peppers (all), grapes & 75 & 1.5 & & 3 & 1 & 0 \\
\hline apple, yellow pept & $06 \mathrm{ft}$ & 100 & 2 & 001168 & 2 & 0 & 0 & 0 & none & 0 & 0 & & 3 & 1 & 0 \\
\hline Erapes, applesauce & e, stirfry vesgi & 100 & 3 & 001468 & 2 & 2 & 1 & 3 & Erapes, sreen apples & 100 & 3 & & 3 & 1 & 의 국 \\
\hline tangerines & $2 a c$ & 100 & 1 & 001968 & 2 & 2 & 0 & 2 & cuties (2), grapes & 100 & 2 & & 3 & 2 & 0 \\
\hline apples & 1 apple svg le & 50 & 1 & 002068 & 2 & 1 & 0 & 1 & mandarin oranges p: & 100 & 1 & & 3 & 2 & 0 \\
\hline none & & of & 요 & 002168 & 2 & 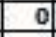 & of & 0 & none & c & 우 & & 3 & 0 & 0 \\
\hline pears & canned in juic & 100 & 1 & 002268 & 2 & 1 & 0 & 1 & pears & 100 & 1 & & 3 & 2 & 0 \\
\hline strawberries & & 90 & 0.9 & 002468 & 2 & 0 & 0 & 0 & veggie chips 1/4 cup & 0 & 요 & & 3 & 2 & 0 \\
\hline none & nolunch & & & 002568 & 2 & 1 & 0 & 1 & strawberries & 100 & 1 & & 3 & 1 & 0 \\
\hline apple & raw, medium & 100 & 1 & $0036 \mathrm{~F}$ & 2 & 1 & 2 & 3 & ves soup with corn a & 100 & 3 & & 3 & 1 & 0 \\
\hline Erapes & Ealso had grap & 80 & 2.18 & $0056 \mathrm{~F}$ & 2 & 1 & of & 1 & Erape juice & 50 & 0.5 & & 3 & 2 & 0 \\
\hline carrots, celery & & 10 & 0.15 & 006 月 & 2 & 인 & 요 & 0 & koolaid cherry juic & 0. & 요 & & 3 & 2 & 요 \\
\hline & & & & 00106 & 2 & & & & & & & & 3 & 2 & 0 \\
\hline Erapes & D6ft & 100 & 1 & 00156 & 2 & 2 & 0 & 2 & applesauce $(0 \%)$, fre & 50 & 1 & & 3 & 1 & 요 \\
\hline applezauce & $06 \mathrm{ft}$ & 100 & 1 & 00166 & 2 & 1 & 0 & 1 & applesauce, also had & 100 & 1 & & 3 & 1 & 0 \\
\hline tangerine & $1 \mathrm{ac}$ & & & 00206 & 2 & 1.5 & 1 & 2.5 & clementine (1) raisis & 100 & 2.5 & & 3 & 2 & 1.5 \\
\hline strawberries, salad & 1 straw, .5 sal & 100 & 2 & 00216 & 2 & 1.3 & 1 & 2.3 & carrots, stawberries & 80 & 1.8 & & 3 & 1 & 0.5 \\
\hline applesauce & to $503.5 \mathrm{oz}$ & 100 & 1 & 00236 & 2 & 1 & 0 & 1 & applesauce & 100 & 1 & & 3 & 1 & 0 \\
\hline applezauce & oift & 100 & 1 & 00246 & 2 & 1 & 이 & 1 & applesauce & 100 & 1 & & 3 & 1 & 욱 \\
\hline none & Oift & of & 아 & 00256 & 2 & 1 & of & 1 & cinnamon applesaud & 100 & 1 & & $3 \mid$ & 1 & 의 \\
\hline
\end{tabular}




\begin{tabular}{|c|c|c|c|c|c|c|c|c|c|c|c|c|c|c|c|}
\hline Numb & Types of serv & $\% \mathrm{com}$ & est c & [D & AVG OF THA & AVG OF THA & AVG OF BOTH & EST Consur & 8 Cons: & $\sqrt{10}$ & Survey 1 目 & Q1M: & $02 \mathrm{AL}$ & c & Q3 \\
\hline & unsweetened & 100 & 2 & 00268 & 1.3333333 & 0.6666667 & 2 & 2 & & 00268 & & 1 & 3 & & 4 \\
\hline 0.25 & tomatoes:1/8 & 100 & 0.3 & $0036 \mathrm{~B}$ & 0.66666667 & 0.5 & \begin{tabular}{|l|}
1.166666667 \\
\end{tabular} & 1.1666667 & 1.17 & $0036 \mathrm{~B}$ & & 1 & 3 & & 4 \\
\hline 1 & applesauce & 100 & 1 & $0046 \mathrm{~B}$ & 1 & 0.1666667 & 1.166666667 & 1.1666667 & 1.17 & 00468 & & 1 & 3 & & 4 \\
\hline 0 & none & 0 & 0 & $0076 \mathrm{~B}$ & 0 & 0 & 0 & 0 & 0 & $0076 B$ & $4 / 13 / 17 ;$ & 1 & 3 & & 2 \\
\hline & applesauce & 0 & 0 & $0096 \mathrm{~B}$ & 0.6666667 & 0.6666667 & \begin{tabular}{|l|}
1.333333333 \\
\end{tabular} & 0.75 & 0.5 & $0096 \mathrm{~B}$ & & 2 & 3 & & 4 \\
\hline & apple & 100 & 1 & 00116 & 0.6666667 & 0,3333333 & 1 & 1 & 1 & $00116 \mathrm{~B}$ & & 1 & 3 & & 4 \\
\hline \begin{tabular}{|l|}
1.25 \\
\end{tabular} & strawberries, & 100 & 1.3 & 00146 B & 1.75 & 0.6666667 & 2.416666667 & 2.4166667 & 1.42 & $00146 B$ & & 2 & 3 & & 4 \\
\hline 1 & apples & 100 & 1 & 00166 & 1.3333333 & 0 & 1.333333333 & 1.3333333 & 1.33 & $00166 \mathrm{~B}$ & ER & & & & \\
\hline 1.5 & Erapes 3/4 cu & 100 & 1.5 & 00196 & 15 & 0 & 1.5 & 1.5 & 1.5 & $00196 \mathrm{~B}$ & & 2 & 3 & & 4 \\
\hline 1.5 & apple 1.5 & 100 & 1.5 & 002067 & 1.5 & 0 & 1.5 & 1.1666667 & 1.17 & $00206 \mathrm{~B}$ & & 1 & 3 & & 4 \\
\hline of & none & 0 & 0 & 00216 & 0 & 0 & of & 0 & 0 & $00216 \mathrm{~B}$ & & 2 & 5 & & 3 \\
\hline 1.5 & perrs, manda & 100 & 1.5 & 002261 & 1.1666667 & 0 & 1.1666666667 & 1.1666667 & 1.17 & $00226 \mathrm{~B}$ & & 1 & 3 & & 4 \\
\hline 1.5 & applesauce, n & 100 & 1.5 & 002466 & 0.8333333 & 0 & 0.833333333 & 1 & 0.5 & $00246 \mathrm{~B}$ & & 2 & 3 & & 4 \\
\hline 1 & Erapes & 100 & 1 & 002565 & 1 & 0 & 1 & 1 & 1 & 002568 & & & & & \\
\hline 0 & none & 0 & 0 & 00156 - & 1.8333333 & 2,3333333 & 4.166666667 & 0.6666667 & 0.67 & $00156 B$ & & 2 & 0 & & 4 \\
\hline 0 & none & 0 & 0 & 001268 & 0.8333333 & 0 & 0.833333333 & 1.92 & 0.83 & 001268 & & 2 & 3 & & 4 \\
\hline 0.25 & lettuce & 100 & 0.3 & 00165 & 0.5 & 0.25 & 0.75 & 0.3333333 & 0.83 & $0016 \mathrm{~F}$ & & 2 & 3 & & 4 \\
\hline 1 & clementines & 100 & 1 & 00265 & 1 & 0 & 1 & 0.7 & 0.83 & $0026 \mathrm{~F}$ & & 1 & 3 & & 4 \\
\hline 1 & blueberries & 80 & 0.8 & 00365 & 11 & 0.6666667 & $1.666666667 \mathrm{7}$ & 1 & 0.83 & $0036 \mathrm{~F}$ & & 2 & 0 & & 4 \\
\hline 2 & erapes 1 cup & 0 & 0 & 00565 & 18933333 & 0 & 1.9 & 1.6 & 0.83 & $0056 F$ & & 2 & 3 & & 4 \\
\hline 2 & watermellon, & 50 & 1 & 0066 & 0.5 & 0.5 & 1.166666667 & 0.8933333 & 0.83 & $0066 \mathrm{~F}$ & & 1 & 3 & & 4 \\
\hline 2 & fruit cup, mal & 100 & 2 & 00106 & 2 & 0 & 1 & 2 & 0.83 & 001067 & & 1 & 3 & & 4 \\
\hline 1 & mandarin ora & 100 & 1 & 00156 & 1.3333333 & 0 & \begin{tabular}{|l|}
1.333333333 \\
\end{tabular} & 0.6666667 & 0.83 & 001567 & & 1 & 6 & & 4 \\
\hline 1 & applesauce & 100 & 1 & 00166 & 1 & 0 & 1 & 1 & 0.83 & 0016 67 & & 1 & 3 & & 4 \\
\hline 3.5 & banana 1 mes & 100 & 3.5 & 00206 & 1.3333333 & 0.8333333 & 2.1666666667 & 1 & 0.83 & 002067 & & 2 & 3 & & 4 \\
\hline 1 & strawberries & 100 & 1 & 00216 & 1.25 & 0.5 & 1.75 & 2 & 0.83 & 00216 F & & 1 & 匀 & & 4 \\
\hline 1 & applesauce & 100 & 1 & 00236 & 1 & 0 & 1 & 1.6 & 0.83 & 002367 & & 2 & 3 & & 4 \\
\hline 1 & applesauce & 100 & 1 & 00246 & 1 & 0 & 1 & 1 & 0.83 & 002465 & & 2 & 6 & & 3 \\
\hline 1 & fruit cooktail & 100 & 1 & 00256 & 0.6666667 & 0 & \begin{tabular}{|l|}
0.666666667 \\
\end{tabular} & 1 & 0.83 & 002567 & & 1 & 3 & & 3 \\
\hline
\end{tabular}

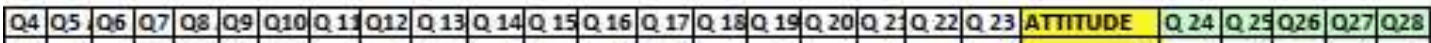

\begin{tabular}{|c|c|c|c|c|c|c|c|c|c|c|c|c|c|c|c|c|c|c|c|c|c|c|c|c|c|c|}
\hline 3 & 3 & 4 & 3 & 3 & 4 & 3 & 4 & 4 & 3 & 3 & 3 & 3 & 2 & 2 & 2 & 3 & 3 & 4 & 3 & 3 & 65 & 2 & 2 & 3 & 1 & 1 \\
\hline 4 & 4 & 4 & 4 & 4 & 4 & 4 & 4 & 4 & 2 & 4 & 3 & 3 & 3 & 3 & 3 & 3 & 3 & 3 & 3 & 3 & 72 & 2 & 2 & 5 & 1 & 1 \\
\hline 4 & 4 & 4 & 4 & 4 & 4 & 4 & 4 & 4 & 4 & 3 & 3 & 4 & 4 & 3 & 3 & 3 & 4 & 3 & 4 & 2 & 76 & 2 & 2 & 3 & 1 & 2 \\
\hline 2 & 3 & 2 & 2 & 2 & 2 & 2 & 3 & 3. & 2 & 3 & 3 & 3 & 2 & 3 & 3 & 3 & 3 & 3 & 3 & 2 & 53 & 2 & 21 & 3 & 1 & 1 \\
\hline 4 & 3 & 3 & 2 & 3 & 2 & 2 & 4 & 4 & 4 & 3 & 2 & 2 & 2 & 3 & 3 & 2 & 1 & 1 & 3 & 2 & 55 & 2 & 2 & 3 & 1 & 2 \\
\hline 4 & 4 & $\overline{4}$ & 4 & 4 & 3 & 4 & 4 & 4 & 3 & 3 & 3 & 3 & 3 & 3 & 3 & 3 & 2 & 2 & 3 & 2 & 68 & 2 & 2 & 5 & 1 & $\overline{1}$ \\
\hline 3 & 3 & 4 & 3 & 4 & 3 & 2 & 4 & 4 & 3 & 3 & 3 & 3 & 3 & 3 & 3 & 4 & 2 & 1 & 4 & 3 & 65 & 2 & 2 & 3 & 1 & 1 \\
\hline & & & & & & & & & & & & & & & & & & & & & & & & & & \\
\hline 3 & 3 & 2 & 2 & 2 & 3 & 3 & 3 & 3 & 3 & 4 & 3 & 3 & 3 & 3 & 2 & 3 & 3 & 3 & 3 & 2 & 60 & 2 & 2 & 3 & 1 & 1 \\
\hline 3 & 2 & 4 & 2 & 2 & 4 & 3 & 4 & 4 & 3 & 2 & 2 & 2 & 1 & 1 & 2 & 2 & 4 & 3 & 3 & 21 & 55 & 2 & 21 & 5 & 1 & 1 \\
\hline 4 & 2 & 4 & 3 & 3 & 4 & 3 & 4 & 4 & 4 & 2 & 3 & 3 & 2 & 3 & 2 & 3 & 4 & 3 & 3 & 2 & 64 & 2 & 2 & 5 & 1 & 1 \\
\hline 3 & 2 & 2 & 3 & 2 & 2 & 3 & 3 & $\underline{3}$ & 1 & 3 & 2 & 2 & 2 & 2 & 2 & 2 & 3 & 3 & 3 & 1 & 50 & 2 & 2 & 4 & 1 & 1 \\
\hline 3 & 3 & 3 & 3 & 3 & 4 & 4 & 4 & 4 & 2 & 4 & 3 & 3 & 3 & 3 & 3 & 3 & 4 & 4 & 4 & 2 & 69 & 2 & 21 & 3 & 1 & 1 \\
\hline & & & & & & & & & & & & & & & & & & & & & & & & 7 & & \\
\hline 3 & 3 & 3 & 3 & 4 & 3 & 3 & 4 & 4 & 3 & 3 & 2 & 2 & 2 & 2 & 2 & 2 & 1 & 1 & 2 & 3 & 55 & 1 & 2 & 5 & 1 & 2 \\
\hline 4 & 2 & 4 & 3 & 3 & 4 & 3 & 3 & 匇 & 2 & 2 & 2 & 2 & 2 & 3 & 2 & 3 & 3 & 3 & 3 & 3 & 60 & 2 & 2 & 3 & 1 & 1 \\
\hline 3 & 3 & 3 & 4 & 4 & 4 & 4 & 4 & 4 & 3 & 4 & 3 & 3 & 4 & 3 & 3 & 4 & 4 & 3 & 4 & 3 & 74 & 2 & 2 & 5 & 1 & 1 \\
\hline 3 & 3 & 4 & 3 & 3 & 4 & 4 & 3 & 匇 & 2 & 3 & 3 & 3 & 2 & 2 & 3 & 3 & 3 & 3 & 3 & 3 & 64 & 2 & 2 & 5 & 1 & 1 \\
\hline 3 & 3 & 2 & 3 & 3 & 4 & 3 & 4 & 4 & 3 & 2 & 3 & 3 & 2 & 2 & 3 & 3 & 2 & 2 & 3 & 3 & 60 & 2 & 2 & 1 & 1 & 1 \\
\hline 4 & 4 & 4 & 4 & 4 & 4 & 4 & 4 & 4 & 5 & 4 & 4 & 4 & 4 & 4 & 4 & 4 & 4 & 4 & 4 & 4 & 85 & 2 & 2 & 3 & 1 & 1 \\
\hline 4 & 3 & 3 & 3 & 3 & 4 & 4 & 3 & 3 & 5 & 2 & 3 & 3 & 3 & 3 & 3 & 3 & 4 & 3 & 4 & 31 & 70 & 3 & 2 & 5 & 1 & 1 \\
\hline 3 & 3 & 3 & 2 & 3 & 3 & 3 & 4 & 4 & 4 & 3 & 3 & 3 & 3 & 3 & 3 & 3 & 3 & 3 & 4 & 3 & 66 & 2 & 2 & 5 & 1 & 1 \\
\hline 4 & 3 & 3 & 3 & 4 & 4 & 3 & 4 & 4 & 2 & 3 & 3 & 3 & 3 & 4 & 4 & 4 & 3 & 3 & 3 & 3 & 70 & 2 & 2 & 5 & 1 & 1 \\
\hline 4 & 3 & 3 & 3 & 4 & 4 & 4 & 4 & 4 & (3) & 3 & 3 & 3 & 3 & 3 & 3 & 3 & 4 & 4 & 4 & 3 & 72 & 2 & 2 & 5 & 1 & 4 \\
\hline 4 & 4 & 4 & 4 & 4 & 4 & 4 & 4 & 4 & 2 & 4 & 4 & 4 & 4 & 4 & 4 & 4 & 4 & 4 & 4 & 3 & 81 & 2 & 2 & 3 & 1 & 1 \\
\hline 4 & 3 & 4 & 4 & 4 & 4 & 4 & 4 & 4 & 4 & 3 & 4 & 4 & 4 & 4 & 3 & 4 & 4 & 4 & 4 & 3 & 80 & 2 & 2 & 3 & 1 & 3 \\
\hline 3 & 2 & 2 & 1 & 3 & 1 & 2 & 4 & 4 & 4 & 1 & 1 & 1 & 2 & 1 & 1 & 3 & 1 & 1 & 3 & 2 & 43 & 2 & 2 & 1 & 1 & 1 \\
\hline 3 & 3 & 2 & 1 & 4 & 1 & 2 & 3 & 3 & 3 & 4 & 4 & 4 & 4 & 3 & 3 & 3 & 4 & 3 & 4 & 4 & 65 & 2 & 21 & 5 & 1 & 4 \\
\hline 3 & 2 & 3 & 2 & 3 & 3 & 4 & 4 & 4 & 2 & 3 & 3 & 3 & 2 & 2 & 2 & 3 & 3 & 3 & 3 & 3 & 59 & 2 & 2 & 1 & 1 & 3 \\
\hline
\end{tabular}




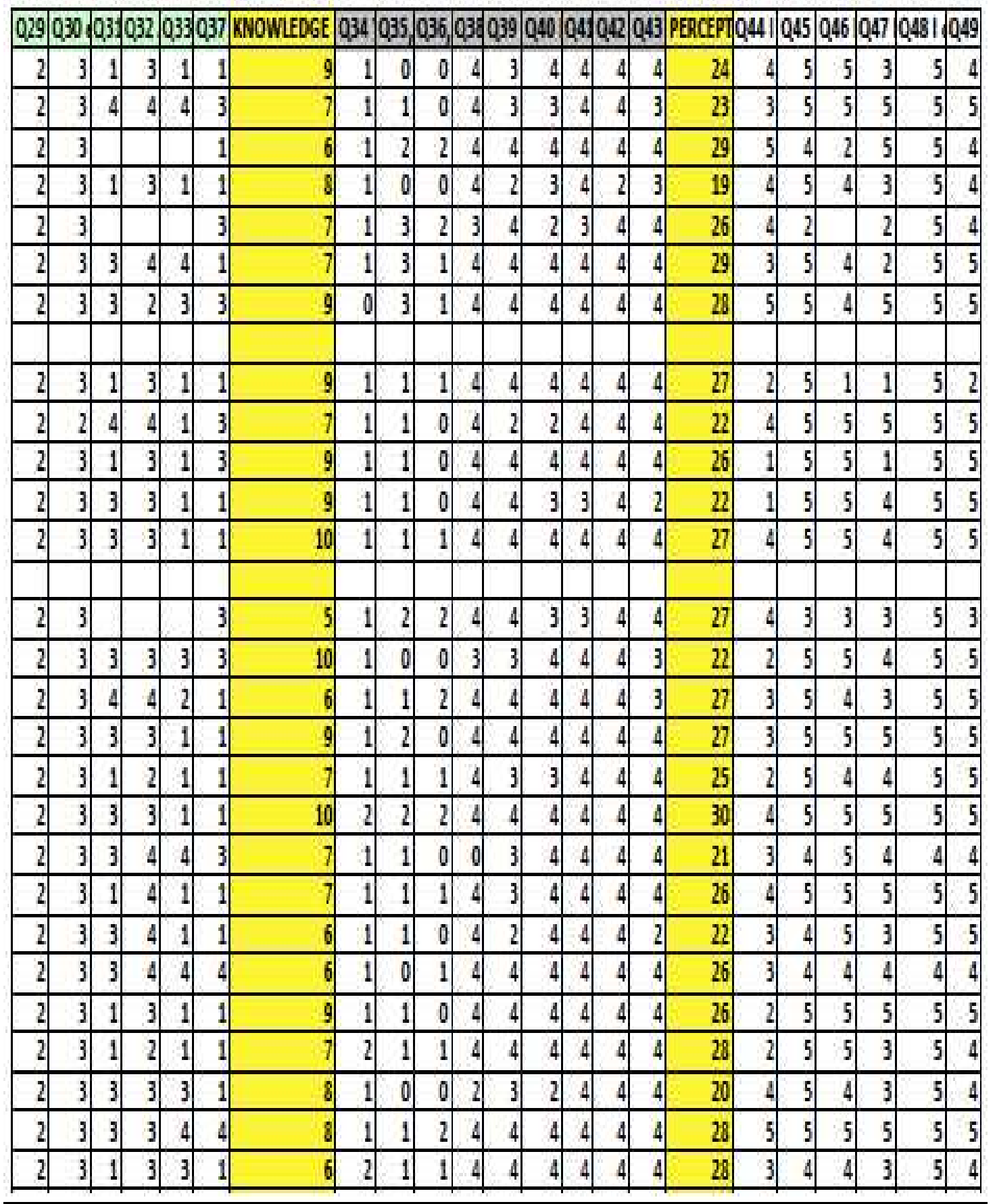




\begin{tabular}{|c|c|c|c|c|c|c|c|c|c|c|c|c|c|c|c|c|c|c|c|c|c|c|}
\hline 96 & & & & 60 & & 7 KNOWLEDGE 0 & & Q35. & Q35. & & $039 \mid \mathrm{C}$ & 9401 & 44 & & 443 & PERCEPTO & Q44I & Q45 & Q46 & Q47 & 1048110 & \\
\hline 2 & 3. & 1 & 3 & 1 & 1 & 9 & 1 & 0 & 0 & 4 & 3] & 4 & 4 & 4 & 4 & 24 & 4 & 5 & 5 & 3 & 5 & 4 \\
\hline 2 & 3 & 4 & 4 & 4 & 3 & 7 & 1 & 1 & 0 & 4 & 79 & 3 & 4 & 4 & 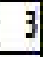 & 23 & 3. & 5 & 5 & 5 & 5 & I \\
\hline 2 & 3 & & & & 1 & 6 & 1 & 2 & 2 & 4 & 4 & 4 & 4 & 4 & 4 & 29 & 5 & 4 & 2 & $d$ & 5 & \\
\hline 2 & 3 & 1 & 3 & 1 & 1 & 8 & 1 & 0 & 0 & 4 & 2 & 3 & 4 & 2 & 3 & 19 & 4 & 5 & 4 & 3 & 5 & 4 \\
\hline 2 & 3 & & & & 3 & 7 & 1 & 3 & 2 & 3 & 4 & 2 & 3 & 4 & 4 & 26 & 4 & 2 & & 2 & 5 & 4 \\
\hline 2 & 3 & 3 & 4 & 4 & 1 & 7 & 1 & 3 & 1 & 4 & 4 & 4 & 4 & 4 & 4 & 29 & 3 & 5 & 4 & 2 & 3 & J \\
\hline 2 & 3 & 3 & 2 & 3 & 3 & 9 & 0 & 3 & 1 & 4 & 4 & 4 & 4 & 4 & 4 & 20. & 5 & 5 & 4 & 5 & 5 & 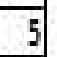 \\
\hline & & & & & & & & & & & & & & & & & & & & & & \\
\hline 2 & 3 & 1 & 3 & 1 & 1 & 9 & 1 & 1 & 1 & 4 & 4 & 4 & 4 & 4 & 4 & 27 & 2 & 5 & 1 & 1 & 5 & 2 \\
\hline 2 & 2 & 4 & 4 & 1 & 3 & 7 & 1 & 1 & 0 & 4 & 2 & 2 & 4 & 4 & 4 & 22 & 4 & 5 & 5 & 5 & 5 & 5 \\
\hline 2 & 3 & 1 & 3 & 1 & 3 & 9 & 1 & $\mathbb{1}$ & 0 & 4 & 4 & 4 & 4 & 4 & 4 & 26 & 1 & 5 & 5 & 1 & 5 & 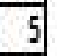 \\
\hline 2 & 3 & 3 & 3 & 1 & 1 & 9 & 1 & 1 & 0 & 4 & 4. & 3 & 3 & 4 & 2 & 22 & 1 & 5 & 5 & 4 & 5 & 5 \\
\hline 2 & 3 & 3 & 3 & 1 & 1 & 10 & 1 & 1 & 1 & 4 & 4 & 4 & 4 & 4 & 4 & 27 & 4 & 5 & 5 & 4 & 5 & 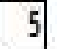 \\
\hline & & & & & & & & & & & & & & & & & & & & & & \\
\hline 2 & 3 & & & & 3 & 5 & 1 & 2 & 2 & 4 & 4 & 3 & 3 & 4 & 4 & 27 & 4 & 3 & 3 & 3 & 5 & 3 \\
\hline 2 & 3 & 3 & 3 & 3 & 3 & 10 & 1 & 0 & 0 & 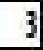 & 3 & 4 & 4 & 4 & 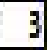 & 22 & 2 & 1 & 5 & 4 & 5 & 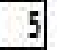 \\
\hline 2 & 3 & 4 & 4 & 2 & 1 & 6) & 1 & 1 & 2 & 4 & 4 & 4 & 4 & 4 & 3 & 27 & 3 & 5 & 4 & 3 & 5 & 5 \\
\hline 2 & 3 & 3 & 3 & 1 & 1 & 9 & 1 & 2 & 0 & 4 & 4 & 4 & 4 & 4 & 4 & 27 & 3 & 5 & 5 & 5 & 5 & 5 \\
\hline 4 & 3 & 1 & 2 & 1 & 1 & 7 & 1 & 1 & 1 & 4 & 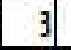 & 1) & 4 & 4 & 4 & 25 & 2 & 5 & 4 & 4 & 5 & 5 \\
\hline 2 & 3 & 3 & 3 & 1 & 1 & 10 & 2 & 2 & 2 & 4 & 4 & 4 & 4 & 4 & 4 & 30 & 4 & 5 & 5 & 5 & 5 & 5 \\
\hline 2 & 3 & 3 & 4 & 4 & 3 & 7 & 1 & 1 & 0 & 0 & 3 & 4 & 4 & 4 & 4 & 21 & 3 & 4 & 5 & 4 & 4 & 4 \\
\hline 2 & 3 & 1 & 4 & 1 & 1 & 7 & 1 & 1 & 1 & 4 & 3 & 4 & 4 & 4 & 4 & 26 & 4 & 5 & 5 & 5 & 5 & 5 \\
\hline 2 & 3 & 3 & 4 & 1 & 1 & 6 & 1 & 1 & 0 & 4 & 2 & 4 & 4 & 4 & 2 & 22 & 3 & 4 & 5 & 3 & 5 & $\partial$ \\
\hline 2 & 3 & 3 & 4 & 4 & 4 & 6 & 1 & 0 & 1 & 4 & 4 & 4 & 4 & 4 & 4 & 26 & 3 & 4 & 4 & 4 & 4 & 4 \\
\hline 2 & 3 & 1 & 3 & 1 & 1 & 9 & 1 & 1 & 0 & 4 & 4 & 4 & 4 & 4 & 4 & 26 & 2 & 5 & 5 & 5 & 5 & 5 \\
\hline 2 & 3 & 1 & 2 & 1 & 1 & 7 & 2 & 1 & 1 & 4 & 4 & 4 & 4 & 4 & 4 & 28 & 2 & 5 & 5 & 3 & 5 & 4 \\
\hline 2 & 3 & 3 & 3 & 3 & 1 & 8 & 1 & 0 & 0 & 2 & 3 & 2 & 4 & 4 & 4 & 20 & 4 & 5 & 4 & 3 & 5 & 4 \\
\hline 2 & 3 & 3 & 3 & 4 & 4 & 8 & 1 & 1 & 2 & 4 & 4 & 4 & 4 & 4 & 4 & 28 & 5 & 5 & 5 & 5 & 5 & 5 \\
\hline 7 & 3 & 1 & 3 & 3 & 1 & 6 & 2 & 1 & 1 & 4 & 4 & 4 & 4 & 4 & 4 & 28 & 3 & 4 & 4 & 3 & 5 & \\
\hline
\end{tabular}




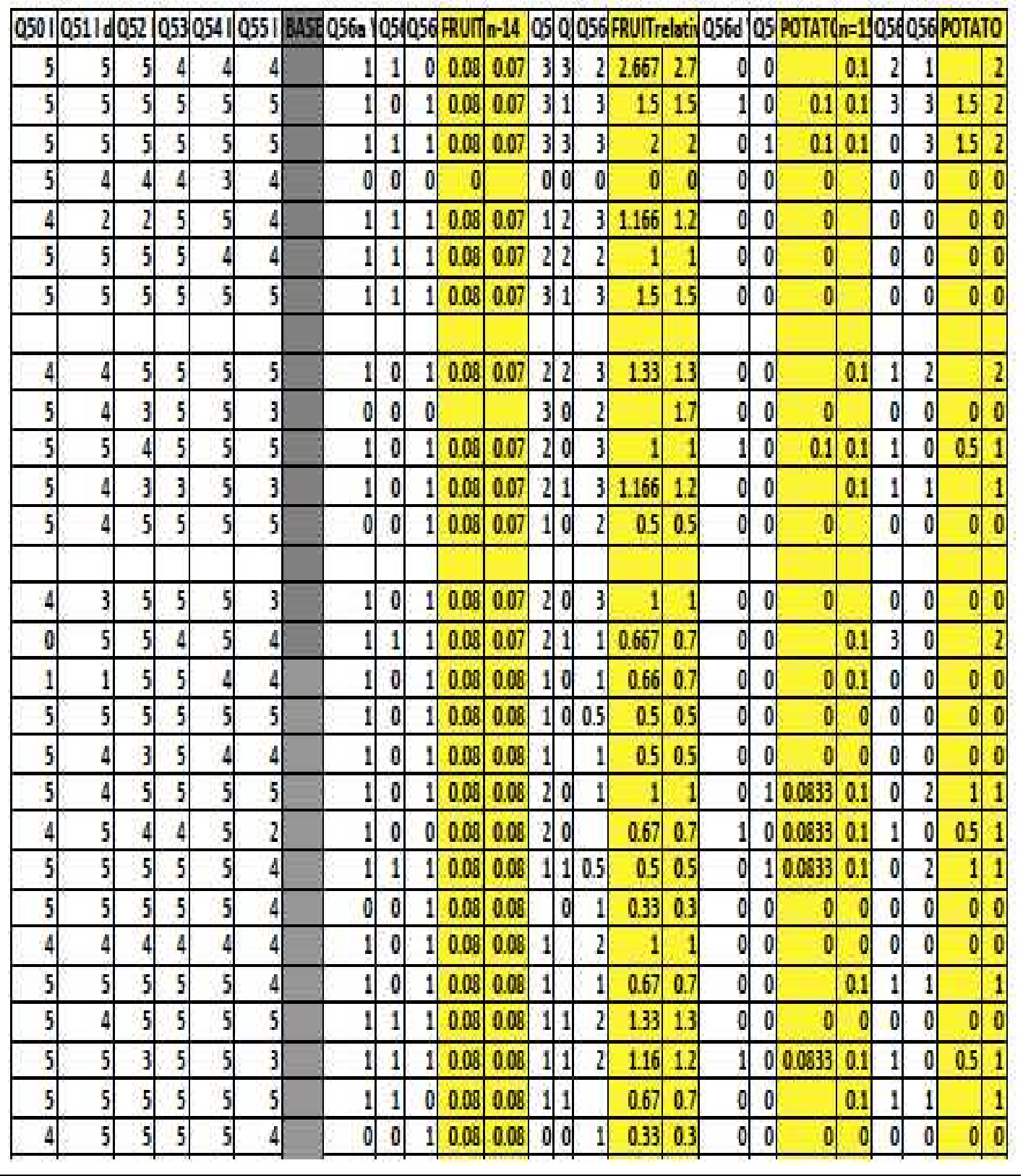




\begin{tabular}{|c|c|c|c|c|c|c|c|c|c|c|c|c|c|c|c|c|c|c|}
\hline $6505: 090$ & & Q59VE & $n=15$ & 056410 & bit & & 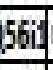 & & 6kik A litt & thte=1, & (566) & 056 & EGL & $n-15$ & $056 \mathrm{~mm}$ & 05 & $\pi$ & \\
\hline $\begin{array}{llllll}1 & 0 & 1 & 1 \\
\end{array}$ & & 0.0 .07 & 0.067 & 3 & & 2 & 2 & 1 & & 1.33 & 1 & 00 & 0077 & 0.07 & & 3 & & 1.5 \\
\hline 0.010 & 0 & & 0.067 & & & 3 & & & & 0.5 & 0 & 0 & 0 & 0 & & & & 0 \\
\hline 10000 & 0 & 0.071 & 0.067 & 3] & & (0) & & & 0.5 & 05 & 1 & 10 & 0.077 & 0.07 & 3. & 3 & 12 & 1.5 \\
\hline 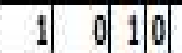 & 0 & $\begin{array}{ll}0.007 \\
0.07\end{array}$ & 0.067 & 2 & 0 & 2 & (1) & 0 & 0.0 .67 & 0.57 & 0 & 0 & 0 & 0) & 0 & 0 & & \\
\hline 100010 & & 0.071 & 40.067 & 0 & & 0 & & & (1) 0 & 0 & 0 & 0 & 0 & 0 & 0 & 0 & & 0 \\
\hline $\begin{array}{lllll}0 & 1 & 0 & 1\end{array}$ & 0 & 0.071 & 0.067 & 0 & 1 & 0 & & 0 & $0[0.33$ & 0.33 & 0 & 0 & 0 & 0 & 0) & 0 & & 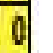 \\
\hline \begin{tabular}{|l|l|l|l}
1 & 1 & 1 & 1
\end{tabular} & 0 & 0.071 & 0.067 & 1 & & 2 & & 0 & 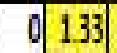 & & 1 & 00 & 0.077 & 0.07 & d) & 2 & & \\
\hline & & & & & & & & & & & & & & & & & & \\
\hline 0.000 & & 0.07 & 0.067 & & & & & & & 0.83 & 0 & 0 & & 0.07 & 2 & & & \\
\hline 0.01010 & 0 & 0.071 & 0.067 & 0 & & 0 & & & 00 & 0 & 0 & 0 & 0 & 4 & d) & 0 & & \\
\hline $1 \quad 1000$ & 0 & 0.007 & 0.067 & 4 & 3 & 0 & 요 & 0 & 0.0 .67 & 0.67 & 0 & 0 & 0 & of & 0 & 0 & & I \\
\hline 10000 & 1 & 0.071 & 0.067 & 2 & & & & & 0.67 & 0.57 & 0 & 0 & & 0.07 & 1 & 1 & & \\
\hline 0.100 & 1 & 0.071 & 0.067 & 0 & 2 & 0 & i] & & 0.0 .67 & 0.57 & 0 & 0 & 0 & 0 & 0 & 0 & & 0 \\
\hline & & & & & & & & & & & & & & & & & & \\
\hline 0.1000 & 0 & 0.071 & 0.067 & 0 & 2 & (0) & 0 & & (a) 0.33 & 0.33 & 0 & 0 & 0 & 0 & 0 & 0 & & 0 \\
\hline $\begin{array}{llll}0 & 1 & 0 & 0 \\
\end{array}$ & 1 & 0.071 & 0.067 & 4 & 2 & 0 & (0) & & \begin{tabular}{l|l|}
0 & 0.5 \\
\end{tabular} & 0.5 & 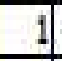 & & 0.07 & 0.07 & ] & 0 & & \\
\hline $\begin{array}{llllll}1 & & 1 & 1 & 1 \\
\end{array}$ & 0 & 0.078 & 0.077 & 2 & 3 & 1 & 3 & 0 & \begin{tabular}{l|l|}
0 & 1.41 \\
\end{tabular} & 1.4 & 0 & 0 & 0 & 0 & d] & 0 & & 0 \\
\hline \begin{tabular}{ll|lll}
0 & 1 & 1 & 10 \\
\end{tabular} & 0 & 0.007 & 0.077 & 0 & 2 & 0 & - & & \begin{tabular}{l|l|}
0.38 \\
\end{tabular} & 0.38 & of & 0 & 0 & 0 & t) & 0 & & 0 \\
\hline $\begin{array}{llll}0 & 0 & 0 & 0\end{array}$ & 1 & 0.007 & 0.077 & 0] & a & 0 & ] & & & 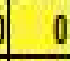 & 0 & 0 & 0 & 0 & d] & 0 & & \\
\hline $1 \quad 1000$ & 1 & 10.076 & 0.077 & 1 & 2 & 0 & - & 3 & 21.33 & 1.33 & 1 & & 0.077 & 0.0 & 1 & 0 & 0. & 0.5 \\
\hline $\begin{array}{lllll}1 & 0 & 0 & 0 \\
\end{array}$ & 0 & 0.076 & 0.077 & 2 & 0 & 0 & d & & 0.033 & 0.33 & of & 0 & o & 0 & d & 0 & & ( \\
\hline \begin{tabular}{|l|l|l|l|}
1 & 1 & 0 & 1
\end{tabular} & 1 & 0.078 & 0.077 & 1 & 2 & 0 & 1 & & 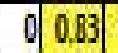 & 0.83 & 0) & 0 & 0 & 0) & 0 & 0 & & \\
\hline 0.0110 & 1 & 0.0 .078 & 0.077 & 0 & 0 & 1 & 0 & 3 & \begin{tabular}{l|l|l|}
0 & 0.58 \\
\end{tabular} & 0.58 & 0) & 0 & 0 & 0 & 0) & 4 & & . \\
\hline $\begin{array}{llll}0 & 0 & 0 & 0 \\
\end{array}$ & 1 & 10.076 & 0.077 & 0 & 0 & 0 & 0 & & & 0.66 & 0 & 10 & 0.077 & 0.08 & 0 & 2 & & 1 \\
\hline 0.01010 & 1 & 0.076 & 0.077 & & & & & 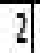 & & 0.33 & 0) & 0 & 0 & 0] & & & & 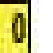 \\
\hline 0.0010 & 1 & 0.0 .076 & 0.077 & 0 & d) & 0 & 0 & 1 & \begin{tabular}{l|l|l|}
0 & 0.16 \\
\end{tabular} & 0.16 & 1 & & 0.077 & 0.00 & 1 & (1) & 0. & 05 \\
\hline $\begin{array}{llll}1 & 0 & 0 & 0 \\
\end{array}$ & 4 & 0.0 .076 & 0.077 & 2 & & 0 & & 0. & \begin{tabular}{l|l|l|}
0 & 0.33 \\
\end{tabular} & 0.33 & 0. & 0 & 0 & 0 & 0 & 0. & & 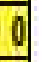 \\
\hline $\begin{array}{lllll}0 & 0 & 0 & 1\end{array}$ & 0 & 0.076 & 9.0077 & & & & 3] & & & 0.5 & 0 & 0 & 0 & 0 & & & & 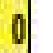 \\
\hline $\begin{array}{llllll}0 & 0 & 1 & 1 & 1 \\
\end{array}$ & 1 & 10.076 & 0.077 & a) & a) & & 3 & & 10.92 & 0.92 & 0. & 0 & ] & 0 & d & & & \\
\hline
\end{tabular}




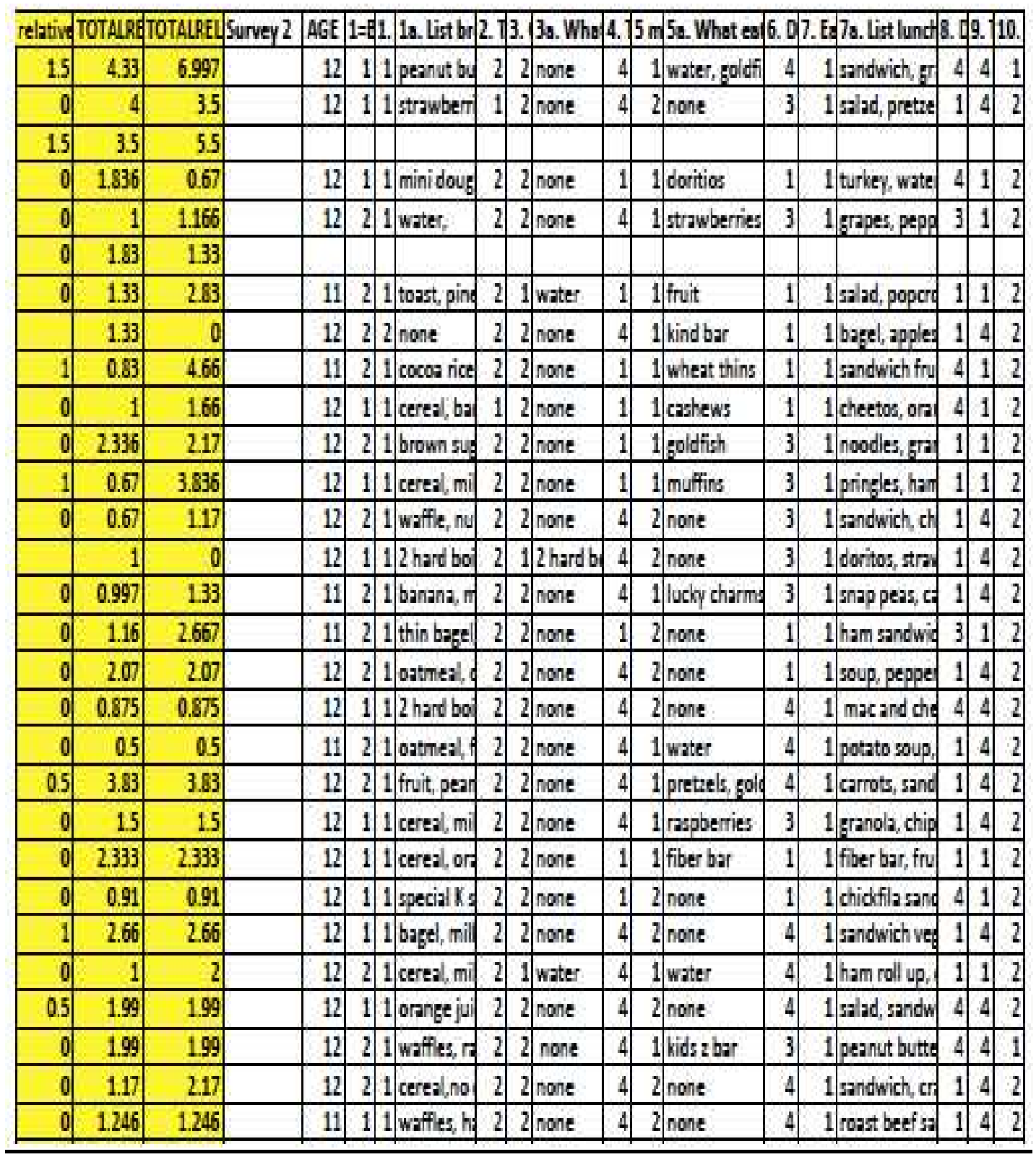




\begin{tabular}{|c|c|c|c|c|}
\hline 10a. Wh & 11. a & 12. & 12a. List snach & SCORE \\
\hline Eranala & 1 & 1 & orange juice & 4 \\
\hline none & 2 & 1 & water & 2 \\
\hline & & & & \\
\hline nane & 1 & 1 & crumstick wate & 0 \\
\hline none & 2 & 1 & pear & 4 \\
\hline none & 1 & 1 . & smoothe, pea & 5 \\
\hline none & 1 & 1 & emoothie & 2 \\
\hline none & 2 & 1 & Eranala bar & 3 \\
\hline none & 1 & 1 & yogurt smooth & 2 \\
\hline none & 1. & 1 & ice crea $m$, fruid & 1 \\
\hline nane & 1 & 1 & poutarts & 1 \\
\hline nane & 1 & 1 & toast & 0 \\
\hline none & 4 & 1 & chicken nusse & 1 \\
\hline none & 2 & 1 & strawberries, c & 5 \\
\hline none & 2 & 1 & strawberries, h & 2 \\
\hline none & 1 & 1 & Foldfish & 2 \\
\hline none & 1 & 1 & pesnut butter & 2 \\
\hline none & 2 & 1 & chacolate ban: & 3 \\
\hline none & 2 & 1 & fruit smoothie & 4 \\
\hline none & 1 & 1 & trquitos & 1 \\
\hline nane & 1 & 1 & crackers & 2 \\
\hline none & 1 & 1 & yogurt, chocol & 1 \\
\hline none & 2 & 1 & Emoothie cars & 2 \\
\hline none & 2 & 1 & banana, apple, & 5 \\
\hline none & 1 & 1 & Pernut butter & 3 \\
\hline water & 2 & 1 & chocolate chip & 2 \\
\hline none: & 2 & 1 & cheerios, string & 1 \\
\hline nane & 2 & 1 & Franola bar & 1 \\
\hline
\end{tabular}




\section{Transcription Group 1: Intervention Group}

\section{PERCEIVED SUSCEPTIBILITY CONSTRUCT}

Moderator: It is April 12 and we are at Immaculate Heart of Mary School with 6B

And we are going to go ahead and get some information about nutrition issues, so go ahead and kind of talk a little bit louder so we can hear.

One of the first questions I want to hear about is "Why do you think some people eat healthy and why do you think people might not eat healthy foods?"

Girl A: I feel like people don't eat healthy foods because sometimes it (the food) doesn't always taste very good, and sometimes people (think) they might be more expensive too.

Moderator: Ok

Girl B: Following up with what said, normally worse foods for you sometimes taste better, but a lot of good foods for you also taste better and .....

Girl C: oh um sometimes like when sometimes when people don't eat healthy, the healthy foods sometimes don't taste as well and the junk food you really like, like there's many different flavors of that stuff

Moderator: so there are some things about taste

Boy A: oh and if you eat healthier there are health benefits

Moderator: Ok, Do you want to talk about some of those

Boy B: living longer

Boy C: Some people eat more healthy, because maybe they're like for instance an actor they have to be more healthy; they have a certain role they have to be a certain weight or look a certain weight.

Moderator: OK

Girl D: Healthy foods also allow you to stay fit and do more physical activities

Girt E: like when you eat sugar like junk food, when you keep eating it like its you crave it, then when you eat a vegetable it just doesn't taste good cause your not used to it and it doesn't taste as good

\section{PERCEIVED SEVERITY CONSTRUCT}

Moderator: so when you are eating fruits and vegetables are you eating healthy or not eating healthy? 
Many: Healthy

Girl E: Like fruits can have sugar, it depends on like what you add to them if they have natural sugar it is good for you like the vegetables that are normally good for you but there are also vegetables like include having vitamins in them

Girl : I also think it depends on how you get them like if they are canned, they might not be as healthy for you

Moderator: Do you think you will have any health problems if you don't eat healthy?

Many: Yes

Boy D: some fruits and vegetables can protect you against diseases so if you don't eat those it might be easier to get that disease

Moderator: Ok and Do you eat fruits and vegetables?

Many: Yes

Moderator: Ok, sometimes...that's fine

Many: lots of chatter (carrots, strawberries, watermelon)

\section{PERCEIVED BENEFITS CONSTRUCT}

Moderator: Are you eating it because they are healthy for you?

Many: I eat fruits because I like them

Boy E: I eat vegetables because my mom makes me

Moderator: You guys talked about health benefits, do you want to talk about the benefits you mentioned cancer prevention, anything else you want to tell me about

Girl CS: um so like Health benefits right? Fruits and vegetables just make you healthier and more fit so it helps you like if you go on a run or something the fruits and vegetables would help you

Girl (MP): They also like give you vitamins that help you do different tasks

Girl (GL) The vegetables, if you eat healthy it helps you do other things like around the house or at your job or something. IF you eat healthy sometimes it is easier to different jobs 


\section{PERCEIVED BARRIERS CONSTRUCT}

Moderator: so maybe, physically and mentally

And how about you all, do you go shopping with your parents?

Many: Yes, every time,

Boy A: Costco is the best

Moderator: do you buy fruits and vegetables when you go?

Are there specific ones you would mention?

Many: apples, blackberries, raspberries, blueberries,

Moderator: Some people do not eat fruits and vegetables do you think they will have poor health?

Many; Yes

\section{CUES TO ACTION CONSTRUCT}

Moderator: ok if yes, what could they do to prevent any health problems or what health problems could they have?

Girl(GL): maybe like Start eating fruits and vegetables and maybe like start working out because its not just eating its combing the physical component

Boy (JL): Any you could get cancer from not eating fruits and vegetables

Boy (JS): High cholesterol maybe?

Moderator: and do you ever cook with recipes that use fruits and vegetables?

Many: Yes;

Girl (GL): We have to have some type of vegetable

Apple Salad

Fruit salad

Girl GL: some things that have sugar in them kind of knocks it out 
Boy JS: or like shakes or

Boy JL protein shakes

\section{SELF-EFFICACY CONSTRUCT}

Moderator: Do you think if you had recipes you would eat more fruits and vegetables if you had different ways to prepare them

Many: yeah

Wait what was the question

Moderator: if you had more recipes for fruits and vegetables do you think that would make you try things more

Many: yes

Moderator: or you could do things with your parents

Girl CS: First it's different recipes and cause like there would be first its different recipes and you also get to try different foods that are involved mixed in the fruits and vegetables maybe you've never tried before and you could really like that

Girl GL: Like if there was a bunch of recipes, like a website or something that had a lot of options or that tasted good, but also healthy for you because taste is like the main component

Moderator: Great you all have lots of ideas to share and that is really great; and really helpful I really appreciate it. Does anyone have anything to add before we wrap up?

Boy JL: I think with recipes that have fruits and vegetables like on their own or if you put it in a sandwich or I don't know then you might grow to like them the fruit and vegetable part.

Moderator: Ok great. Well great, thank you I think you had a lot of great ideas I think this will be a lot of helpful information as we go forward with trying to plan more education materials and such for other kids that are your age across Indianapolis and the state. It is really valuable and thank you so much for taking the time to do this.

Many: thank you 


\begin{tabular}{|c|c|}
\hline $\begin{array}{l}\text { HEALTH } \\
\text { BELIEF } \\
\text { MODEL } \\
\text { CONSTRUCT }\end{array}$ & $\begin{array}{l}\text { SELECTED DISCUSION PROBES AND EMERGING } \\
\text { THEME FROM FOCUS GROUP }\end{array}$ \\
\hline $\begin{array}{l}\text { Perceived } \\
\text { susceptibility of } \\
\text { poor health } \\
\text { related to lack } \\
\text { of fruits and } \\
\text { vegetables in } \\
\text { your diet } \\
\text { CODING: }\end{array}$ & $\begin{array}{l}\text { Why do you think some people eat healthy and why do some } \\
\text { people not eat healthy foods? If you eat fruits and vegetables } \\
\text { are you eating healthy or not eating healthy? Will you have } \\
\text { health problems if you don't eat healthy? } \\
\text {-Taste } \\
\text {-Cost } \\
\text {-Health benefits }\end{array}$ \\
\hline $\begin{array}{l}\text { Perceived } \\
\text { severity of poor } \\
\text { health related to } \\
\text { low intake of } \\
\text { fruits and } \\
\text { vegetables } \\
\text { CODING: }\end{array}$ & $\begin{array}{l}\text { Do you eat fruits and vegetables? If yes, do you eat them } \\
\text { because they are good for you? } \\
\text {-Living longer } \\
\text {-Appearance } \\
\text {-Weight management } \\
\text {-Fitness } \\
\text {-Energy level } \\
\text {-Natural sugars } \\
\text {-Vitamins }\end{array}$ \\
\hline $\begin{array}{l}\text { Perceived } \\
\text { benefits of fruits } \\
\text { and vegetables } \\
\text { CODING: }\end{array}$ & $\begin{array}{l}\text { What are the health benefits of fruit and vegetables foods? Do } \\
\text { you eat fruits and vegetables for the health benefits? } \\
\text {-PREVENT CANCER } \\
\text {-RUN MORE }\end{array}$ \\
\hline $\begin{array}{l}\text { Perceived } \\
\text { barriers of } \\
\text { consuming } \\
\text { fruits and } \\
\text { vegetables } \\
\text { CODING: }\end{array}$ & $\begin{array}{l}\text { Do you go food shopping with our parents? Do you buy fruits } \\
\text { and vegetables when you go shopping? Why or why not? } \\
\text {-YES } \\
\text {-ALL TYPES }\end{array}$ \\
\hline $\begin{array}{l}\text { Cues to Action } \\
\text { for health } \\
\text { prevention }\end{array}$ & $\begin{array}{l}\text { Some people do not eat any fruits and vegetables or they eat } \\
\text { low amounts. Do you think that they will have poor health? If } \\
\text { yes, what can they do to help prevent any health problems. } \\
\text { What kind of health problems might they have? } \\
\text {-YES } \\
\text {-HIGH CHOLESTEROL } \\
\text {-CANCER }\end{array}$ \\
\hline
\end{tabular}




\begin{tabular}{|l|l|}
\hline $\begin{array}{l}\text { Self-efficacy for } \\
\text { consuming } \\
\text { fruits and } \\
\text { vegetables }\end{array}$ & $\begin{array}{l}\text { Do you every cook recipes that have fruits and vegetables as } \\
\text { ingredients? Do you think if you had recipes with fruits and } \\
\text { vegetables as ingredients would you eat more fruits and } \\
\text { vegetables? }\end{array}$ \\
CODING: & $\begin{array}{l}\text {-YES } \\
\text {-PUT IT WITH SOMETHING ELSE } \\
\text {-GROW TO LIKE THEM; TASTE IMPROVED }\end{array}$ \\
\hline
\end{tabular}

\section{Transcription 2: Control Group}

Okay it is Wednesday April $12^{\text {th }}$ and this is $6 \mathrm{~F}$ group

\section{PERCEIVED SUSCEPTIBILITY CONSTRUCT}

Moderator: so we will go ahead and get started

So again this is about nutrition, and I want to ask some questions. Why do you think some people eat healthy, and why do you think some people eat unhealthy foods?

Go ahead

Girl MG: so some people might not eat healthy foods because like their surroundings are influencing them, like the people around them don't eat healthy foods either

Moderator: go ahead

Boy JM: I know that some people do eat healthy foods because they know that they will help them and its also a way that they were taught that healthy foods are good for you.

Boy: it kind of depends on your job, like if you are an athlete you need to eat good foods.

Girl AO: um I feel that sometimes more people are more motivated to eat more healthy foods like if that's just part of their lifestyle eating healthy foods, then its probably easier for them to eat more things that are healthier

Girl KC: maybe some people haven't gotten the chance to eat healthy foods so sometimes they just eat the foods that they know.

Boy AH: kind of adding to , some people they might have tried healthy foods they don't like and there is more to try but they just haven't had the chance to.

Boy : yeah I was going to say what he said

Girl Some people would eat healthy foods because they know it will benefit them in the activities that they do, because they know it will keep them energized instead of eating foods that are not as good for you.

Moderator: do you think they wouldn't be energized or 
Girl :: Sometimes I know that you can get those energy drinks that have a lot of sugar in them, but they have make you like bouncing of the walls hyper, so maybe if they knew that you could be energized by eating in a healthier way they might try it.

Boy WP: Even if I don't like the vegetable or fruit, I would have it because it would help me be healthier.

Boy TD: people might like they might eat a non-healthy food because they might not be informed that a healthy food helps you more.

Boy CW: They might not eat healthy because their parents don't like forcing it upon them, but if they tell them it is good for you, they would probably eat more

Boy JM: So like the people might eat non healthy foods because that's what they've done all the time and its just something they've always done; they really haven't really eaten healthy foods some they have always eaten healthy foods and they just don't eat much of the non-healthy foods

Girl: Like their parents aren't going to make them eat healthy foods so they eat unhealthy foods because they like the taste better.

Girl MR: At the store, healthy foods are more typically than unhealthy foods so people might pick the cheaper option.

Moderator: Ok, so if you eat fruits and vegetables do you think you are eating healthy or not eating healthy?

Boy AH: um, um with fruits I mean like your kind of like both, some of it has sugar and sugar is helping you with it and its not like real sugar that's in like all of the junk and sodas.

Girl KC: I think that eating fruits and vegetables is eating healthy because it gives you like vitamins that you need to grow.

Boy WP: well um for like um fruits and vegetables I know that like candy and like unhealthy things have sugar and like all that bad extra calories that don't help you. I know healthy vegetables have the calories that make you like healthier.

Moderator: Ok

Eating fruits and vegetables will like help you grow and to grow up and be strong, like as to eating a lot of junk food that won't make you as strong that you need to be.

Girl AO: Um fruits and vegetables when you eat them give you the energy you need to keep going through the day and they help you to get through school or a sport or any 
activity that you need to do. It is good that when you eat fruits and vegetables they will help you when you do those things.

Moderator: ok

Boy WM: this kind of goes with what Caroline said earlier with the energy drinks, that they will get you active and you feel fine but then you will crash eventually, and with fruits and vegetables you don't crash. So...

Boy CW: Like, ah, I forgot.

If you are eating fruits and vegetables are you eating healthy or not eating healthy

Boy CW: it's good that you eat fruits and vegetables, but you can't make up your whole diet with fruits and vegetables, so you need other good stuff.

Boy JM: in both fruits and vegetables and in candies there is sugar, but fruits and vegetables there are vitamins and other nutrients that you can get.

Moderator: and do you think you will have health problems if you don't eat healthy foods?

Boy JM: evidence can be shown that in like old fashioned, old ships where they didn't have much vegetables and they only ate salted meat.

\section{Moderator Ok}

Boy CW: Your not going to get completely cured from eating fruits and vegetables, but it's a lot better if you don't eat candy and stuff like that than fruits and vegetables.

Boy TD: Its ok to eat non healthy foods some times, but if you eat too much of it, then it becomes a problem.

Moderator: and do you eat fruits and vegetables?

Girl: Yes

\section{PERCEIVED SERIOUSNESS CONSTRUCT}

Moderator: and if you do eat them, do you eat them because you think they are good for you or why?

Boy : Well, um my mom she its like the only thing that she eats. She does it cause its' like a habit and I should probably do that more often. 
Boy TD: I haven't probably realized a ton like early on, but mostly my mom makes dinner and I usually haven't realized it, but usually there is a vegetable in it and fruits sometimes you just eat um, because I like fruits a lot.

Moderator: so if you eat fruits and vegetables are you eating them because you think they are good for you?

Boy WP: when I usually eat, I try to balance my meals up so like half of it fruits and vegetable I make it an entrée like chicken.

Girl MG: I think I eat them, because personally I think I like the taste of fruits and vegetables and I'm fine with eating them but I also think I think about I probably should be eating fruits and vegetables with how much activity I do, I probably need something like nutritious to keep me going.

Girl CS: I do eat fruits and vegetables. I probably should eat more though, and I do like the taste of them, but sometimes, I like forget to eat them and I eat other stuff instead.

Girl AO: I eat fruits and vegetables. I like fruits and vegetables, but I also eat them because I need the energy they provide me with, whereas, with other stuff like candy I can't get that. Because with fruits and vegetables they have lots of vitamins and they provide you with energy and they help you do the things you need to get done.

Boy WM : Well I eat fruits and vegetables, but I feel like with fruit during the day can just go grab a fruit like orange or apple and eat that, but I won't really do that with vegetables unless its' like on my plate.

Boy CW: um, with like we always at fruits and vegetables like with dinner we always have like two or three. And then after dinner we usually have like a fruit.

Boy JM: Um like my mom always has like a fruit for lunch and vegetable for dinner and I always eat that; and sometimes like throughout the day I just eat pickles or cucumber. It's not just because I know that they are good for me, its just that I have always eaten them and I just they taste good.

\section{PERCEIVED BENEFITS CONSTRUCT}

Moderator: so what are the health benefits of fruits and vegetables?

Girl CS: they will give you more energy than like other unhealthy things.

Boy JM: they give you nutrients that are necessary for a healthy body and life

Boy CW: um, they like, give you more energy.

Boy WM: kind of like J said, they give you vitamins that you need. 


\section{PERCEIVED BARRIERS CONSTRUCT}

Girl AO : they provide you they sustain the energy whereas they just don't give you energy for about an hour or two then they stop they help you throughout the day and even going forward they sustain the energy.

Girl AK: they provide a healthy diet in the right way and eating fruits and vegetables can help because it keeps your energy up, but not like decreased it just keeps going.

Girl MG: I don't know specifically, but I know some fruits and vegetables help with eyesight and so like eating fruits and vegetables is important because overall it keeps your body healthy.

\section{CUES TO ACTION CONSTRUCT}

Moderator: and do you go grocery shopping with your parents?

Many yes

Boy JM: my mom usually does it while we are at school, because she has work to do Moderator: and if you do go, do you buy fruits and vegetables when you go shopping ?

Many : yes

Boy WM: my mom usually lets me pick and I pick pears.

Moderator: and so there are some people that do not eat any fruits or vegetables or they might eat very low amounts of them, so do you think that they would have poor health?

Many : Yes

Boy AH: yeah if that's the only thing that they would eat healthy and like with all of the activities you do, like $\mathrm{W}$ plays hockey and you need a lot of energy for that, because your going to be moving like every play.

\section{SELF-EFFICACY CONSTRUCT}

Moderator: and do you think if um they do help prevent healthy problems, what kind of health problems might they have?

Girl CS: Like there energy, they would have energy, but their energy might not be the right type of energy. Like if you eat fruits and vegetables you just keep energy and like if you drink an energy drink you might just crash and you won't have any energy in you.

Moderator: any health specific..

Boy TD : I think you could get dehydrated cause I feel like fruit has a lot of juices in them and unless you drink a lot of water you need fruit. 
Boy WM: I don't think you would necessarily have to have health problems or have to have them all of the time cause you can get the some vitamins and nutrition from different things but they're usually like the best way to get them.

Boy JM: um if you don't eat enough fruits and vegetables then you can get blood cells and veins that would be blocked and also like I said you can get survey or loose your teeth and get black skin.

Moderator: and do you use recipes that have fruits and vegetables as ingredients?

Many: yes

Moderator: any examples or anything you want to share about that?

Girl KC: My mom makes this pot roast that has carrots, broccoli and potatoes in it .

Boy AH: my dad eats like um, I don't know what its called but it's like a beef stew and he makes really good chili AND HE DOES CARROTS, celery, broccoli and some meat to balance it out. And I don't know if this counts but he does some spaghetti with the pasta sauces.

Boy TD: my mom sometimes makes banana bread and puts bananas in it.

Moderator: Ok

Boy WP: Um, chicken potpie, she puts like carrots, peas, and like chicken in it.

Moderator: :Ok

Girl MG: My mom makes this like pasta and she puts like all these different kinds of vegetables in it and she also like our pasta sauce, she doesn't tell us, but she blends it with fruits and vegetables, not fruits, but vegetables and doesn't tell us and it tastes the same and we usually eat it.

Girl CS: My dad also he like makes stir fry and he puts a lot of veggies in it like meat in there and he also makes kabobs that you can like grill with meat on it with a bunch of vegetables and stuff like that.

Girl AO; A lot of times my mom will make a salad with a lot of vegetables and she will sometimes makes soup that has a bunch of carrots and broccoli and like corn made like that.

Boy WM: Well my mom makes lots of stuff with vegetables in it for some reason I don't know but my younger sister really likes making fruit salad so its just like strawberries and stuff like that. And we have that a lot. 
Boy CW: um, my parents like to make fried rice a lot and we just put in broccoli and peas, corn, a lot of stuff in.

Boy JM: in my family we have a lot of noodle stuff and my mom she just got this new noodle maker, so instead of making noodles it makes zoodles so it is noodles made out of zucchini and they are actually really good.

Girl ED: My mom makes chicken noodle soup and she puts like a lot of carrots, peas, corn and other vegetables in it.

Girl MR: my mom makes enchiladas and um corn, beans, chicken, carrots, great.

Moderator: and do you think if you have more recipes that you would, with fruits and vegetables, that you would eat more fruits and vegetables?

Many: yes!

We would make that a lot

Moderator: you would try things?

Girl MG: I think you would because like sometimes it's harder to eat them by itself and with other things you might like them better and with other things you might like them easier.

Girl CS: Sometimes the taste is better with a lot of other ingredients, because some people don't always like eating it like M said, and just straight without anything and like sometimes it needs to be mixed with other things.

Moderator: ok

Girl AO: sometimes its hard to get recipes with all of fruits and vegetables so I feel like you would be more motivated to have them if you had a bunch of recipes. And um you would want to try them more and uh yeah.

Boy WM: I kind of feel like I'll eat them if they re part of the dinner, but I usually just don't go take them for a snack, like what I said earlier.

(can you say the question again)

Moderator: yeah, if you had more recipes with fruits and vegetables, as ingredients would you eat more fruits and vegetables?

Girl kind of like what all these three said, if you want it or with something 
Girl AO: Sometimes also I don't know if this is just me, but you feel more proud and want to eat it if its something that you made and might be motivation for people if they had a lot of recipes too.

Boy WM: and if like your tastes buds are always changing so if you have meals with vegetables you could try something new that you find out that you like or you could figure out that you like it.

Moderator: any last thoughts before we wrap up?

Boy AH: In California, by mom didn't want the buns so mom got the protein one and she had a lettuce wrap instead of the buns around it and she liked that a lot .

Moderator: um, yeah a different way to have it.

Girl MG: Um, I think that when you put fruits and vegetables with other things there a lot more options and with only just fruits and vegetables there are a lot less, because there are only certain kinds of fruits and vegetable so when you put them with recipes you can have a lot more choices of things you like.

Girl CS: Like if you have more recipes, you have more selection, and like choices and if you don't like a certain vegetable maybe its in a dish that you really like, maybe you could try it and you didn't know it could be done.

Moderator: those are all wonderful thoughts, thank you so much for sharing all of those with me. I think I got a lot of great ideas so thank you. 


\begin{tabular}{|c|c|}
\hline Health Belief Model Construct & Selected Discussion Probes \\
\hline $\begin{array}{l}\text { Perceived susceptibility of poor health } \\
\text { related to lack of fruits and vegetables in } \\
\text { your diet }\end{array}$ & $\begin{array}{l}\text { Why do you think some people eat healthy } \\
\text { and why do some people not eat healthy } \\
\text { foods? If you eat fruits and vegetables are } \\
\text { you eating healthy or not eating healthy? } \\
\text { Will you have health problems if you don't } \\
\text { eat healthy? } \\
\text {-Surroundings; motivation; energy; } \\
\text { taught that way/education; lifestyle; job } \\
\text { requirement; health }\end{array}$ \\
\hline $\begin{array}{l}\text { Perceived severity of poor health related to } \\
\text { low intake of fruits and vegetables } \\
\text { CODING: }\end{array}$ & $\begin{array}{l}\text { Do you eat fruits and vegetables? If yes, do } \\
\text { you eat them because they are good for } \\
\text { you? } \\
\text {-my mom makes me; balance meals }\end{array}$ \\
\hline $\begin{array}{l}\text { Perceived benefits of fruits and vegetables } \\
\text { CODING: }\end{array}$ & $\begin{array}{l}\text { What are the health benefits of fruit and } \\
\text { vegetables foods? Do you eat fruits and } \\
\text { vegetables for the health benefits? } \\
\text {-prevent cancer; run more; nutrients; } \\
\text { healthy body }\end{array}$ \\
\hline $\begin{array}{l}\text { Perceived barriers of consuming fruits and } \\
\text { vegetables } \\
\text { CODING: }\end{array}$ & $\begin{array}{l}\text { Do you go food shopping with our parents? } \\
\text { Do you buy fruits and vegetables when you } \\
\text { go shopping? Why or why not? } \\
\text {-many yes; yes; many parents work and } \\
\text { it is worked in }\end{array}$ \\
\hline Cues to Action for health prevention & $\begin{array}{l}\text { Some people do not eat any fruits and } \\
\text { vegetables or they eat low amounts. Do you } \\
\text { think that they will have poor health? If } \\
\text { yes, what can they do to help prevent any } \\
\text { health problems? What kind of health } \\
\text { problems might they have } \\
\text {-energy; blood health and skin; vitamins } \\
\text { and nutrients gained }\end{array}$ \\
\hline $\begin{array}{l}\text { Self-efficacy for consuming fruits and } \\
\text { vegetables }\end{array}$ & $\begin{array}{l}\text { Do you every cook recipes that have fruits } \\
\text { and vegetables as ingredients? Do you } \\
\text { think if you had recipes with fruits and } \\
\text { vegetables as ingredients would you eat } \\
\text { more fruits and vegetables? } \\
\text {-more choices; more ideas; pride in } \\
\text { making own dishes; involve the child; } \\
\text { taste }\end{array}$ \\
\hline
\end{tabular}




\section{CHAPTER VIII}

\section{REFERENCES}

Abi Haidar, G., Lahham Salameh, N., \& Afifi, R. A. (2011). Jarrib Baleha--a pilot nutrition intervention to increase water intake and decrease soft drink consumption among school children in Beirut. J Med Liban, 59(2), 55-64.

Abrams, S. A., Griffin, I. J., Hawthorne, K. M., Liang, L., Gunn, S. K., Darlington, G., \& Ellis, K. J. (2005). A combination of prebiotic short- and long-chain inulin-type fructans enhances calcium absorption and bone mineralization in young adolescents. Am J Clin Nutr, 82(2), 471-476.

Abuajah, C. I., Ogbonna, A. C., \& Osuji, C. M. (2015). Functional components and medicinal properties of food: a review. J Food Sci Technol, 52(5), 2522-2529. doi:10.1007/s13197-014-1396-5

Ameratunga, R., Crooks, C., Simmons, G., \& Woon, S. T. (2016). Health Risks and Adverse Reactions to Functional Foods. Crit Rev Food Sci Nutr, 56(2), 318-325. doi:10.1080/10408398.2012.751895

Anderson, E. S., Winett, R. A., \& Wojcik, J. R. (2007). Self-regulation, self-efficacy, outcome expectations, and social support: social cognitive theory and nutrition behavior. Ann Behav Med, 34(3), 304-312. doi:10.1080/08836610701677659

Anderson-Bill, E. S., Winett, R. A., \& Wojcik, J. R. (2011). Social cognitive determinants of nutrition and physical activity among web-health users enrolling in an online intervention: the influence of social support, self-efficacy, outcome expectations, and self-regulation. J Med Internet Res, 13(1), e28. doi:10.2196/jmir.1551

Annesi, J. J., \& Mareno, N. (2014). Temporal aspects of psychosocial predictors of increased fruit and vegetable intake in adults with severe obesity: mediation by physical activity. J Community Health, 39(3), 454-463. doi:10.1007/s10900-0149828-8

Annesi, J. J., \& Vaughn, L. L. (2017). Directionality in the Relationship of Selfregulation, Self-efficacy, and Mood Changes in Facilitating Improved Physical Activity and Nutrition Behaviors: Extending Behavioral Theory to Improve Weight-Loss Treatment Effects. J Nutr Educ Behav, 49(6), 505-512.e501. doi:10.1016/j.jneb.2017.03.004

Ashford, J. B., \& Lecroy, C. W. (2010). Human behavior in the social environment: A multidimensional perspective (4th ed.). Belmont, CA.

Bandura, A. (1994). Self-Efficacy. In V.S. Ramachaudran (Ed.), (Vol. 4). New York: Academic Press.

Bannon, K., \& Schwartz, M. B. (2006). Impact of nutrition messages on children's food choice: pilot study. Appetite, 46(2), 124-129. doi:10.1016/j.appet.2005.10.009

Baranowski, T. (2006). Advances in basic behavioral research will make the most important contributions to effective dietary change programs at this time. $\mathrm{J} \mathrm{Am}$ Diet Assoc, 106(6), 808-811. doi:10.1016/j.jada.2006.03.032 
Baranowski, T., Davis, M., Resnicow, K., Baranowski, J., Doyle, C., Lin, L. S., . . . Wang, D. T. (2000). Gimme 5 fruit, juice, and vegetables for fun and health: outcome evaluation. Health Educ Behav, 27(1), 96-111.

Baranowski, T., Watson, K. B., Bachman, C., Baranowski, J. C., Cullen, K. W., Thompson, D., \& Siega Riz, A. M. (2010). Self efficacy for fruit, vegetable and water intakes: Expanded and abbreviated scales from item response modeling analyses. Int J Behav Nutr Phys Act, 7, 25. doi:10.1186/1479-5868-7-25

Bauer, U. E., Briss, P. A., Goodman, R. A., \& Bowman, B. A. (2014). Prevention of chronic disease in the 21 st century: elimination of the leading preventable causes of premature death and disability in the USA. Lancet, 384(9937), 45-52. doi:10.1016/s0140-6736(14)60648-6

Bazzano, L. A. (2006). The high cost of not consuming fruits and vegetables. $J$ Am Diet Assoc, 106(9), 1364-1368. doi:10.1016/j.jada.2006.06.021

Beecher, G. R. (1998). Nutrient content of tomatoes and tomato products. Proc Soc Exp Biol Med, 218(2), 98-100.

Bentley, G. F., Goodred, J. K., Jago, R., Sebire, S. J., Lucas, P. J., Fox, K. R., . . Turner, K. M. (2012). Parents' views on child physical activity and their implications for physical activity parenting interventions: a qualitative study. BMC Pediatr, 12, 180. doi:10.1186/1471-2431-12-180

Birch, L., Savage, J. S., \& Ventura, A. (2007). Influences on the Development of Children's Eating Behaviours: From Infancy to Adolescence. Can J Diet Pract Res, 68(1), s1-s56.

Biro, L., Regoly-Merei, A., Nagy, K., Peter, S., Arato, G., Szabo, C., . . Antal, M. (2007). Dietary habits of school children: representative survey in metropolitan elementary schools. Part two. Ann Nutr Metab, 51(5), 454-460. doi:10.1159/000111166

Bonvecchio, A., Theodore, F. L., Safdie, M., Duque, T., Villanueva, M. A., Torres, C., \& Rivera, J. (2014). Contribution of formative research to design an environmental program for obesity prevention in schools in Mexico City. Salud Publica Mex, 56 Suppl 2, s139-147.

Borradaile, K. E., Foster, G. D., May, H., Karpyn, A., Sherman, S., Grundy, K., . . . Boruch, R. F. (2008). Associations between the Youth/Adolescent Questionnaire, the Youth/Adolescent Activity Questionnaire, and body mass index z score in low-income inner-city fourth through sixth grade children. Am J Clin Nutr, 87(6), 1650-1655.

Boulton, J., Hashem, K. M., Jenner, K. H., Lloyd-Williams, F., Bromley, H., \& Capewell, S. (2016). How much sugar is hidden in drinks marketed to children? A survey of fruit juices, juice drinks and smoothies. BMJ Open, 6(3). doi:10.1136/bmjopen-2015-010330

Brown, J. (Ed.) (2013). Nutrition through the lifecycle, 5th ed (5th ed.). Belmont, CA.

Brown, L., Poudyal, H., \& Panchal, S. K. (2015). Functional foods as potential therapeutic options for metabolic syndrome. Obes Rev, 16(11), 914-941. doi:10.1111/obr.12313

Brown, R., \& Ogden, J. (2004). Children's eating attitudes and behaviour: a study of the modelling and control theories of parental influence Health Educ Res, 19(3), 261271. 
Brownell, K. D., Kelman, J. H., \& Stunkard, A. J. (1983). Treatment of obese children with and without their mothers: changes in weight and blood pressure. Pediatrics, 71(4), 515-523.

Burke, L. E., Dunbar-Jacob, J., Orchard, T. J., \& Sereika, S. M. (2005). Improving adherence to a cholesterol-lowering diet: a behavioral intervention study. Patient Educ Couns, 57(1), 134-142. doi:10.1016/j.pec.2004.05.007

Burnet, D., Plaut, A., Courtney, R., \& Chin, M. H. (2002). A practical model for preventing type 2 diabetes in minority youth. Diabetes Educ, 28(5), 779-795. doi: $10.1177 / 014572170202800519$

Burrows, T., Warren, J. M., Baur, L. A., \& Collins, C. E. (2008). Impact of a child obesity intervention on dietary intake and behaviors. International Journal of Obesity, 32(10), 1481-1488.

Cain, E. (2011). Starch grains on neandertal teeth debunks theory that dietary deficiences caused their extinction. Retrieved from http://insider.si.edu/2011/01/starchgrains-found-on-neandertal-teeth-helps-debunk-theory-their-extinction-wascaused-by-dietary-deficiencies/

Casagrande, S. S., Wang, Y., Anderson, C., \& Gary, T. L. (2007). Have Americans increased their fruit and vegetable intake? The trends between 1988 and 2002. Am J Prev Med, 32(4), 257-263. doi:10.1016/j.amepre.2006.12.002

CDC. (2008). Childhood obesity facts. Retrieved from https://www.cdc.gov/obesity/data/childhood.html

CDC. (2014). Children eating more fruit, but fruit and vegetable intake still too low. Retrieved from http://www.cdc.gov/media/releases/2014/p0805-fruitsvegetables.html

CDC. (2016). Growth charts. Retrieved from http://www.cdc.gov/growthcharts/data tables.htm

CDC. (2017). BMI percentile calculator for child and teen. . Retrieved from https://nccd.cdc.gov/dnpabmi/calculator.aspx

CDC (Producer). (n.d., October 1, 2017). Public health surveillance if fruit and vegetable intake using the behavioral risk factor surveillance system.

Cengage. (2004). Tolerable upper intake levels for vitamins and minerals. Retrieved from http://www.cengage.com/nutrition/discipline content/tables/0-534-5589867 C.pdf

Charach, A., Volpe, T., Boydell, K. M., \& Gearing, R. E. (2008). A theoretical approach to medication adherence for children and youth with psychiatric disorders. Harv Rev Psychiatry, 16(2), 126-135. doi:10.1080/10673220802069715

Cheng, F., Monnat, S., \& Lohse, B. (2015). Middle School-Aged Child Enjoyment of Food Tastings Predicted Interest in Nutrition Education on Osteoporosis Prevention. Journal of School Health, 85(7), 467-476. doi:10.1111/josh.12268

Clark, M. M., Abrams, D. B., Niaura, R. S., Eaton, C. A., \& Rossi, J. S. (1991). Selfefficacy in weight management. J Consult Clin Psychol, 59(5), 739-744.

Clemens, R., Drewnowski, A., Ferruzzi, M. G., Toner, C. D., \& Welland, D. (2015). Squeezing fact from fiction about 100\% fruit juice. Adv Nutr, 6(2), 236s-243s. doi:10.3945/an.114.007328 
Corbi, G., Conti, V., Davinelli, S., Scapagnini, G., Filippelli, A., \& Ferrara, N. (2016). Dietary Phytochemicals in Neuroimmunoaging: A New Therapeutic Possibility for Humans? Front Pharmacol, 7, 364. doi:10.3389/fphar.2016.00364

Costello, R., Wallace, T. C., \& Rosanoff, A. (2016). Magnesium. Adv Nutr, 7(1), 199201. doi:10.3945/an.115.008524

Cree-Green, M., Triolo, T. M., \& Nadeau, K. J. (2013). Etiology of insulin resistance in youth with type 2 diabetes. Curr Diab Rep, 13(1), 81-88. doi:10.1007/s11892012-0341-0

Creswell, J. (2008). Educational research: planning, conducting, and evaluating quantitative and qualitative research (3rd ed.). New Jersey: Pearson Education, Inc.

Crittenden, A. N., \& Schnorr, S. L. (2017). Current views on hunter-gatherer nutrition and the evolution of the human diet. Am J Phys Anthropol, 162 Suppl 63, 84-109. doi:10.1002/ajpa.23148

Cullum-Dugan, D., \& Pawlak, R. (2015). REMOVED: Position of the Academy of Nutrition and Dietetics: Vegetarian Diets. Journal of the Academy of Nutrition and Dietetics, 115(5), 801-810. doi:http://dx.doi.org/10.1016/j.jand.2015.02.033

Das, J. K., Salam, R. A., Thornburg, K. L., Prentice, A. M., Campisi, S., Lassi, Z. S., . . . Bhutta, Z. A. (2017). Nutrition in adolescents: physiology, metabolism, and nutritional needs. Ann N Y Acad Sci, 1393(1), 21-33. doi:10.1111/nyas.13330

Dauchet, L., Amouyel, P., \& Dallongeville, J. (2009). Fruits, vegetables and coronary heart disease. Nature Reviews Cardiology, 6, 599+.

Delahanty, L., Kriska, A., Edelstein, S., Amodei, N., Chadwick, J., Copeland, K., . . . Mayer-Davis, E. (2013). Self-reported dietary intake of youth with recent onset of type 2 diabetes: results from the TODAY study. J Acad Nutr Diet, 113(3), 431439. doi:10.1016/j.jand.2012.11.015

DeNavas-Walt, C., \& Protor, B. (2014). Income and poverty in the United States: 2013. United States Census Bureau. Retrieved from http://www.census.gov/content/dam/Census/library/publications/2014/demo/p60249.pdf

Dewar, D., Lubans, D., Plotnikoff, R., \& Morgan, P. (2012). Development and evaluation of social cognitive measures related to adolescent dietary behaviors. Int J Behav Nutr Phys Act. doi: 10.1186/1479-5868-9-36

DOE. (2010). Indiana Academic Standards for Health and Wellness. Retrieved from http://www.doe.in.gov/standards/health-and-wellness

Drewnowski, A., \& Eichelsdoerfer, P. (2010). Can Low-Income Americans Afford a Healthy Diet? Nutrition today, 44(6), 246-249. doi:10.1097/NT.0b013e3181c29f79

Drewnowski, A., \& Rehm, C. D. (2015). Socioeconomic gradient in consumption of whole fruit and 100\% fruit juice among US children and adults. Nutr J, 14, 3 . doi:10.1186/1475-2891-14-3

DRI. (2006). DRI essential guide. DRI essential guide. Retrieved from http://www.nap.edu/catalog/11537.html

Durham, W., Sykes, C., Piper, S., \& Stokes, P. (2015). Conceptual frameworks and terminology in doctoral nursing research. Nurse Res, 23(2), 8-12.

doi: $10.7748 / \mathrm{nr} .23 .2 .8 . \mathrm{s} 3$ 
Eckermann, S., Dawber, J., Yeatman, H., Quinsey, K., \& Morris, D. (2014). Evaluating return on investment in a school based health promotion and prevention program: The investment multiplier for the Stephanie Alexander Kitchen Garden National Program. Social Science \& Medicine, 114, 103-112. doi:10.1016/j.socscimed.2014.05.056

Edmunds, L. D., \& Ziebland, S. (2002). Development and validation of the Day in the Life Questionnaire (DILQ) as a measure of fruit and vegetable questionnaire for 7-9 year olds. Health Educ Res, 17(2), 211-220.

Emmett, P. M., \& Jones, L. R. (2015). Diet, growth, and obesity development throughout childhood in the Avon Longitudinal Study of Parents and Children. Nutr Rev, 73 Suppl 3, 175-206. doi:10.1093/nutrit/nuv054

Epstein, L. H., Paluch, R. A., Gordy, C. C., \& Dorn, J. (2000). Decreasing sedentary behaviors in treating pediatric obesity. Arch Pediatr Adolesc Med, 154(3), 220226.

Ernst, J., \& Whelan, J. (2015). Evaluation of fruit and vegetable intake at a private school.

Euling, S. Y., Herman-Giddens, M. E., Lee, P. A., Selevan, S. G., Juul, A., Sorensen, T. I., . . . Swan, S. H. (2008). Examination of US puberty-timing data from 1940 to 1994 for secular trends: panel findings. Pediatrics, 121 Suppl 3, S172-191. doi:10.1542/peds.2007-1813D

Evans, C. E., Christian, M. S., Cleghorn, C. L., Greenwood, D. C., \& Cade, J. E. (2012). Systematic review and meta-analysis of school-based interventions to improve daily fruit and vegetable intake in children aged 5 to 12 y. Am J Clin Nutr, 96(4), 889-901. doi:10.3945/ajcn.111.030270

Faria, A. M., Gomes-Santos, A. C., Goncalves, J. L., Moreira, T. G., Medeiros, S. R., Dourado, L. P., \& Cara, D. C. (2013). Food components and the immune system: from tonic agents to allergens. Front Immunol, 4, 102. doi:10.3389/fimmu.2013.00102

Fenton, N. (2014). The adolescent health belief model. Retrieved from https://cdr.lib.unc.edu/.../uuid:73ac347e-cab6-4190-ac54-87162fd3a

Flegal KM, C. M., Ogden CL, Curtin LR. . (2010). Prevalence and trends in obesity among US adults. JAMA, 303(3), 235-241.

Fugl-Meyer, A. R., Hellström, K., Lindmark, B., \& Wahlberg, B. (2003). Self-Efficacy in Relation to Impairments and Activities of Daily Living Disability in Elderly Patients with Stroke: A Prospective Investigation. Journal of Rehabilitation Medicine, 35(5), 202-207. doi:10.1080/16501970310000836

Gamble, S., Mawokomatanda, T., Xu, F., Chowdhury, P. P., Pierannunzi, C., Flegel, D., . . Town, M. (2017). Surveillance for Certain Health Behaviors and Conditions Among States and Selected Local Areas - Behavioral Risk Factor Surveillance System, United States, 2013 and 2014. MMWR Surveill Summ, 66(16), 1-144. doi:10.15585/mmwr.ss6616a1

Gibson, G., \& Roberfroid, M. (1995). Dietary modulation of the human colonic microbiota: introducing the concept of prebiotics. $J$ Nutr, 125(6), 1401-1412.

Gibson, R. S. (2005). Anthropometric assessment of body composition. Principles of Nutritional Assessment. New York, NY: Oxford University Press Inc. . 
Glanz, K., Rimer, B. K., \& Viswanath, K. (2016). Health behavior and health education: Theory, research, and practice. (4th ed.). San Francisco, CA: Jossey-Bass.

Glasgow, R. E., Toobert, D. J., Mitchell, D. L., Donnelly, J. E., \& Calder, D. (1989). Nutrition education and social learning interventions for type II diabetes. Diabetes Care, 12(2), 150-152.

Guenther, P. M., Dodd, K. W., Reedy, J., \& Krebs-Smith, S. M. (2006). Most Americans eat much less than recommended amounts of fruits and vegetables. $J$ Am Diet Assoc, 106(9), 1371-1379. doi:10.1016/j.jada.2006.06.002

Guetterman, T. C., Fetters, M. D., \& Creswell, J. W. (2015). Integrating Quantitative and Qualitative Results in Health Science Mixed Methods Research Through Joint Displays. Ann Fam Med, 13(6), 554-561. doi:10.1370/afm.1865

Gupta, R. S., Lau, C. H., Warren, C. M., Lelchuk, A., Alencar, A., Springston, E. E., \& Holl, J. L. (2013). The impact of student-directed videos on community asthma knowledge. J Community Health, 38(3), 463-470. doi:10.1007/s10900-012-96304

Haberman, C., Brauer, P., Dwyer, J. J., \& Edwards, A. M. (2014). Self-reported health behaviour change in adults: analysis of the Canadian Community Health Survey 4.1. Chronic Dis Inj Can, 34(4), 248-255.

Hall, E., Chai, W., Koszewski, W., \& Albrecht, J. (2015). Development and validation of a social cognitive theory-based survey for elementary nutrition education program. Int J Behav Nutr Phys Act, 12(1), 47. doi:10.1186/s12966-015-0206-4

Hamman, R. F., Bell, R. A., Dabelea, D., D’Agostino, R. B., Dolan, L., Imperatore, G., . . . Saydah, S. (2014). The SEARCH for Diabetes in Youth Study: Rationale, Findings, and Future Directions. Diabetes Care, 37(12), 3336-3344. doi:10.2337/dc14-0574

Hansen, A. R., Alfonso, M. L., Hackney, A. A., \& Luque, J. S. (2015). Preschool children's self-reports of fruit and vegetable knowledge, preference, and messages encouraging consumption. J Sch Health, 85(6), 355-364. doi:10.1111/josh.12260

Hanson, M. A., \& Gluckman, P. D. (2015). Developmental origins of health and disease - Global public health implications. Best Practice \& Research Clinical Obstetrics \& Gynaecology, 29(1), 24-31. doi:http://dx.doi.org/10.1016/j.bpobgyn.2014.06.007

Hayden, J. (2009). Introduction to Health Behavior Theory. Sudbury, MA: Jones \& Bartlett Publishers.

Helland, S. H., Bere, E., \& Overby, N. C. (2016). Study protocol for a multi-component kindergarten-based intervention to promote healthy diets in toddlers: a cluster randomized trial. BMC Public Health, 16, 273. doi:10.1186/s12889-016-2952-x

Henning, P., Conaway, H. H., \& Lerner, U. H. (2015). Retinoid receptors in bone and their role in bone remodeling. Front Endocrinol (Lausanne), 6, 31. doi:10.3389/fendo.2015.00031

Hess, J. R., Birkett, A. M., Thomas, W., \& Slavin, J. L. (2011). Effects of short-chain fructooligosaccharides on satiety responses in healthy men and women. Appetite, 56(1), 128-134. doi:10.1016/j.appet.2010.12.005

Hong, T., Johnson, C. C., Myers, L., Boris, N., Brewer, D., \& Webber, L. S. (2008). Process evaluation of an in-school anti-tobacco media campaign in Louisiana. Public Health Rep, 123(6), 781-789. 
Hurt, R. T., \& McClave, S. A. (2016). Nutritional Assessment in Primary Care. Medical Clinics of North America, 100(6), 1169-1183. doi:http://dx.doi.org/10.1016/j.mcna.2016.06.001

Ickes, S. B., Trichler, R. B., \& Parks, B. C. (2015). Building a Stronger System for Tracking Nutrition-Sensitive Spending: A Methodology and Estimate of Global Spending for Nutrition-Sensitive Foreign Aid. Food Nutr Bull, 36(4), 520-533. doi:10.1177/0379572115611785

Ingram, J., Cabral, C., Hay, A. D., Lucas, P. J., \& Horwood, J. (2013). Parents' information needs, self-efficacy and influences on consulting for childhood respiratory tract infections: a qualitative study. BMC Fam Pract, 14, 106. doi:10.1186/1471-2296-14-106

IOM. (2001). Dietary Reference Intakes for Vitamin A, Vitamin K, Arsenic, Boron, Chromium, Copper, Iodine, Iron, Manganese, Molybdenum, Nickel, Silicon, Vanadium, and Zinc. Washington, DC: National Academy Press.

IOM. (2017). Dietary reference intakes tables and appliation. Retrieved from http://nationalacademies.org/HMD/Activities/Nutrition/SummaryDRIs/DRITables.aspx

Islam, M. A., Alam, F., Solayman, M., Khalil, M. I., Kamal, M. A., \& Gan, S. H. (2016). Dietary Phytochemicals: Natural Swords Combating Inflammation and Oxidation-Mediated Degenerative Diseases. Oxid Med Cell Longev, 2016, 5137431. doi:10.1155/2016/5137431

Jamelske, E., Bica, L. A., McCarty, D. J., \& Meinen, A. (2008). Preliminary findings from an evaluation of the USDA Fresh Fruit and Vegetable Program in Wisconsin schools. Wmj, 107(5), 225-230.

Jeffery, I. B., \& O'Toole, P. W. (2013). Diet-microbiota interactions and their implications for healthy living. Nutrients, 5(1), 234-252. doi:10.3390/nu5010234

Jenkins, D. J. A., Boucher, B. A., Ashbury, F. D., Sloan, M., Brown, P., El-Sohemy, A., . . . Kreiger, N. (2017). Effect of Current Dietary Recommendations on Weight Loss and Cardiovascular Risk Factors. J Am Coll Cardiol, 69(9), 1103-1112. doi:10.1016/j.jacc.2016.10.089

Jilcott Pitts, S. B., Wu, Q., Demarest, C. L., Dixon, C. E., Dortche, C. J., Bullock, S. L., . .. Ammerman, A. S. (2015). Farmers' market shopping and dietary behaviours among Supplemental Nutrition Assistance Program participants. Public Health Nutr, 18(13), 2407-2414. doi:10.1017/s1368980015001111

Johnson, E., \& Russell, R. (2010). Encyclopedia of Dietary Supplements. 2nd ed. London and New York: Informa Healthcare.

Kay, D., \& Kibble, J. (2016). Learning theories 101: application to everyday teaching and scholarship. Adv Physiol Educ, 40(1), 17-25. doi:10.1152/advan.00132.2015

Kehm, R., Hearst, M. O., Sherman, S., \& Elwell, K. L. (2016). The FAV-S Pilot Study: Increasing Self-Efficacy and Fruit and Vegetable Intake Among Somali Women and Children. Health Educ Behav. doi:10.1177/1090198116646367

Kelder, S. H., Perry, C. L., Lytle, L. A., \& Klepp, K. I. (1995). Community-wide youth nutrition education: long-term outcomes of the Minnesota Heart Health Program. Health Educ Res, 10(2), 119-131.

Kelly, R. K., Thomson, R., Smith, K. J., Dwyer, T., Venn, A., \& Magnussen, C. G. (2015). Factors affecting tracking of blood pressure from childhood to adulthood: 
The childhood determinants of adult health study. The Journal of Pediatrics, 167(6), 1422-1428.

Kim, S. A., Moore, L. V., Galuska, D., Wright, A. P., Harris, D., Grummer-Strawn, L. M., ... Rhodes, D. G. (2014). Vital signs: fruit and vegetable intake among children - United States, 2003-2010. MMWR Morb Mortal Wkly Rep, 63(31), 671676.

Koplan, J. P., Liverman, C. T., \& Kraak, V. I. (2005). Preventing childhood obesity: Health in the balance: Executive summary. J Am Diet Assoc, 105, 131-138.

Krebs-Smith, S. M. K., L.S. (2001). Choose a variety of fruits and vegetables daily: Understanding the complexities. Journal of Nutrition, 131(2S-1), 487S-501S.

Kristo, A. S., Klimis-Zacas, D., \& Sikalidis, A. K. (2016). Protective Role of Dietary Berries in Cancer. Antioxidants (Basel), 5(4). doi:10.3390/antiox5040037

LaBrosse, L., \& Albrecht, J. A. (2013). Pilot intervention with adolescents to increase knowledge and consumption of folate-rich foods based on the Health Belief Model. International Journal of Consumer Studies, 37(3), 271-278. doi:10.1111/ijcs.12004

Larson, N., Davey, C. S., Caspi, C. E., Kubik, M. Y., \& Nanney, M. S. (2017). SchoolBased Obesity-Prevention Policies and Practices and Weight-Control Behaviors among Adolescents. Journal of the Academy of Nutrition and Dietetics, 117(2), 204-213. doi:https://doi.org/10.1016/j.jand.2016.09.030

Lim, S. S., Vos, T., Flaxman, A. D., Danaei, G., Shibuya, K., Adair-Rohani, H., .. . Ezzati, M. (2012). A comparative risk assessment of burden of disease and injury attributable to 67 risk factors and risk factor clusters in 21 regions, 1990-2010: a systematic analysis for the Global Burden of Disease Study 2010. Lancet, 380(9859), 2224-2260. doi:10.1016/S0140-6736(12)61766-8

Lock, K., Pomerleau, J., Causer, L., Altmann, D., \& McKee, M. (2005). The global burden of disease attributable to low consumption of fruit and vegetables: Implications for the global strategy on diet. Bull World Health Organ, 83, 100108.

Lohman, T. G., Roche, A. F., \& al., e. (1988). Anthropometric standardization reference manual, abridged edition. Champaign, IL.: Human Kinetics Books.

Lowry, R., Lee, S. M., McKenna, M. L., Galuska, D. A., \& Kann, L. K. (2008). Weight management and fruit and vegetable intake among US high school students. $J$ Sch Health, 78(8), 417-424; quiz 455-417. doi:10.1111/j.1746-1561.2008.00324.x

Lubans, D. R., Smith, J. J., Plotnikoff, R. C., Dally, K. A., Okely, A. D., Salmon, J., \& Morgan, P. J. (2016). Assessing the sustained impact of a school-based obesity prevention program for adolescent boys: the ATLAS cluster randomized controlled trial. Int J Behav Nutr Phys Act, 13, 92. doi:10.1186/s12966-016-04208

Markowiak, P., \& Slizewska, K. (2017). Effects of Probiotics, Prebiotics, and Synbiotics on Human Health. Nutrients, 9(9). doi:10.3390/nu9091021

Martin, B. R., Braun, M. M., Wigertz, K., Bryant, R., Zhao, Y., Lee, W., . . . Weaver, C. M. (2010). Fructo-oligosaccharides and calcium absorption and retention in adolescent girls. J Am Coll Nutr, 29(4), 382-386.

Martin-Biggers, J., Spaccarotella, K., Hongu, N., Alleman, G., Worobey, J., \& ByrdBredbenner, C. (2015). Translating it into real life: a qualitative study of the 
cognitions, barriers and supports for key obesogenic behaviors of parents of preschoolers. BMC Public Health, 15, 189. doi:10.1186/s 12889-015-1554-3

Martinez de Victoria, E. (2016). Calcium, essential for health. Nutr Hosp, 33(Suppl 4), 341. doi:10.20960/nh.341

Mâsse, L. C., \& de Niet, J. E. (2013). School nutritional capacity, resources and practices are associated with availability of food/beverage items in schools. Int J Behav Nutr Phys Act, 10, 26. doi:10.1186/1479-5868-10-26

Matvienko, O. (2007). Impact of a nutrition education curriculum on snack choices of children ages six and seven years. J Nutr Educ Behav, 39(5), 281-285. doi:10.1016/j.jneb.2007.01.004

MayoClinic. (2016). Nutrition and healthy eating. Retrieved from http://www.mayoclinic.org/healthy-lifestyle/nutrition-and-healthy-eating/indepth/fiber/art-20043983

McGuire, S. (2012). Institute of Medicine (IOM) Early Childhood Obesity Prevention Policies. Washington, DC: The National Academies Press; 2011. Adv Nutr, 3(1), 56-57. doi:10.3945/an.111.001347

Migliozzi, M., Thavarajah, D., Thavarajah, P., \& Smith, P. (2015). Lentil and Kale: Complementary Nutrient-Rich Whole Food Sources to Combat Micronutrient and Calorie Malnutrition. Nutrients, 7(11), 9285-9298. doi:10.3390/nu7115471

Miles, C. L., Matthews, J., Brennan, L., \& Mitchell, S. (2010). Changes in the content of children's school lunches across the school week. Health Promot J Austr, 21(3), 196-201.

Morton, K. L., Atkin, A. J., Corder, K., Suhrcke, M., \& van Sluijs, E. M. (2016). The school environment and adolescent physical activity and sedentary behaviour: a mixed-studies systematic review. Obes Rev, 17(2), 142-158. doi:10.1111/obr.12352

Mukerji, K. (2006). Fruit and vegetable diseases. Delhi, India: Klewer Academic Publishers.

Mulualem, D., Henry, C. J., Berhanu, G., \& Whiting, S. J. (2016). The effectiveness of nutrition education: Applying the Health Belief Model in child-feeding practices to use pulses for complementary feeding in Southern Ethiopia. Ecol Food Nutr, 55(3), 308-323. doi:10.1080/03670244.2016.1161617

Murphy, A. J., White, M., Viani, K., \& Mosby, T. T. (2016). Evaluation of the nutrition screening tool for childhood cancer (SCAN). Clin Nutr, 35(1), 219-224. doi:10.1016/j.clnu.2015.02.009

Musher-Eizenman, D. R., \& Kiefner, A. (2013). Food parenting: a selective review of current measurement and an empirical examination to inform future measurement. Child Obes, 9 Suppl, S32-39. doi:10.1089/chi.2013.0030

Must, A., Spadano, J., Coakley, E. H., Field, A. E., Colditz, G., \& Dietz, W. H. (1999). The disease burden associated with overweight and obesity. JAMA, 282(16), 1523-1529.

Nagy, L. (2012). Would eating carrots protect your liver? A new role involving NKT cells for retinoic acid in hepatitis. Eur J Immunol, 42(7), 1677-1680. doi:10.1002/eji.201242705

NCES. (2013). U.S. Department of Education, National Center for Education Statistics: parent and family involvement in education, from the National Household 
Education Surveys Program of 2012. Retrieved from

https://nces.ed.gov/fastfacts/display.asp?id=91

NCES. (2014). National center for education statistics. Retrieved from http://nces.ed.gov/fastfacts/display.asp?id=372.

Neville, C. E., McKinley, M. C., Draffin, C. R., Gallagher, N. E., Appleton, K. M., Young, I. S., ... Woodside, J. V. (2015). Participating in a fruit and vegetable intervention trial improves longer term fruit and vegetable consumption and barriers to fruit and vegetable consumption: a follow-up of the ADIT study. Int $J$ Behav Nutr Phys Act, 12, 158. doi:10.1186/s12966-015-0311-4

NIH. (2004). Blood pressure tables for children and adolescents. Retrieved from https://www.nhlbi.nih.gov/health-pro/guidelines/current/hypertension-pediatricjnc-4/blood-pressure-tables

NIH. (2007). A pocket guide to blood pressure measurement in children. Retrieved from

NIH. (2016). Fruit and vegetable screeners in the eating at America's table study (EATS): scoring. Retrieved from https://epi.grants.gov/diet/screeners/fruitveg/scoring/alldayscreener

Nixon, C. A., Moore, H. J., Douthwaite, W., Gibson, E. L., Vogele, C., Kreichauf, S., .. . ToyBox-study, g. (2012). Identifying effective behavioural models and behaviour change strategies underpinning preschool- and school-based obesity prevention interventions aimed at 4-6-year-olds: a systematic review. Obesity Reviews, 13 Supplement(1), 106-117.

Noverr, M. C., \& Huffnagle, G. B. (2004). Does the microbiota regulate immune responses outside the gut? Trends in microbiology, 12(12), 562-568.

Ogden, C. L., Carroll, M. D., Kit, B. K., \& Flegal, K. M. (2014). Prevalence of childhood and adult obesity in the United States, 2011-2012. JAMA, 311(8), 806-814. doi:10.1001/jama.2014.732

Olza, J., Aranceta-Bartrina, J., Gonzalez-Gross, M., Ortega, R. M., Serra-Majem, L., Varela-Moreiras, G., \& Gil, A. (2017). Reported Dietary Intake and Food Sources of Zinc, Selenium, and Vitamins A, E and C in the Spanish Population: Findings from the ANIBES Study. Nutrients, 9(7). doi:10.3390/nu9070697

Papizadeh, M., Rohani, M., Nahrevanian, H., Javadi, A., \& Pourshafie, M. R. (2017). Probiotic characters of Bifidobacterium and Lactobacillus are a result of the ongoing gene acquisition and genome minimization evolutionary trends. Microbial Pathogenesis, 111(Supplement C), 118-131. doi:https://doi.org/10.1016/j.micpath.2017.08.021

Pase, M. P., Grima, N., Cockerell, R., \& Pipingas, A. (2015). Habitual intake of fruit juice predicts central blood pressure. Appetite, 84, 68-72. doi:10.1016/j.appet.2014.09.019

Perry, C. P., Keane, E., Layte, R., Fitzgerald, A. P., Perry, I. J., \& Harrington, J. M. (2015). The use of a dietary quality score as a predictor of childhood overweight and obesity. BMC Public Health, 15, 581. doi:10.1186/s12889-015-1907-y

Pliner, P., \& Hobden, K. (1992). Development of a scale to measure the trait of food neophobia in humans. Appetite, 19(2), 105-120.

Popkin, B. M., \& Hawkes, C. (2016). Sweetening of the global diet, particularly beverages: patterns, trends, and policy responses. Lancet Diabetes Endocrinol, 4(2), 174-186. doi:10.1016/s2213-8587(15)00419-2 
Porter, C. C., \& Marlowe, F. W. (2007). How marginal are forager habitats? Journal of Archaeological Science, 34(1), 59-68. doi:http://dx.doi.org/10.1016/j.jas.2006.03.014

Position of ADA, S., \& ASFSA. (2003). Position of the American Dietetic Association, Society for Nutrition Education, and American School Food Service Association: Nutrition services: An essential component of comprehensive school health programs. Journal of Nutrition Education \& Behavior, 35(2), 57-67.

Principi, N., \& Esposito, S. (2016). Gut microbiota and central nervous system development. Journal of Infection. doi:http://dx.doi.org/10.1016/j.jinf.2016.09.010

Robertson, T. P., \& Zalles, D. R. (2005). Nutrition education program Nutrition Pathfinders teaches children how to make healthful food choices. J Nutr Educ Behav, 37(1), 41-42.

Robinson, S., Bielamowicz, M., Rodgers, A., Wong, W., \& Konzelmann, K. (2008). Designing a bone health and soy focus group discussion guide based on the health belief model. Journal of Extension, 46(1).

Roger, V. L., Go, A. S., Lloyd-Jones, D. M., Adams, R. J., Berry, J. D., Brown, T. M., . . . Stroke Statistics, S. (2011). Heart disease and stroke statistics--2011 update: a report from the American Heart Association. Circulation, 123(4), e18-e209. doi:10.1161/CIR.0b013e3182009701

Rogers, E. A., Fine, S. C., Handley, M. A., Davis, H. B., Kass, J., \& Schillinger, D. (2016). Engaging Minority Youth in Diabetes Prevention Efforts Through a Participatory, Spoken-Word Social Marketing Campaign. Am J Health Promot. doi:10.4278/ajhp.141215-ARB-624

Rosi, A., Scazzina, F., Ingrosso, L., Morandi, A., Del Rio, D., \& Sanna, A. (2015). The "5 a day" game: a nutritional intervention utilising innovative methodologies with primary school children. International Journal of Food Sciences \& Nutrition, 66(6), 713-717. doi:10.3109/09637486.2015.1077793

Salihu, H. M., Adegoke, K. K., Das, R., Wilson, R. E., Mazza, J., Okoh, J. O., . . Berry, E. L. (2016). Community-based fortified dietary intervention improved health outcomes among low-income African-American women. Nutr Res, 36(8), 771779. doi:10.1016/j.nutres.2016.04.006

Sanders, M. E. (2015). Probiotics in 2015: Their Scope and Use. J Clin Gastroenterol, 49 Suppl 1, S2-6. doi:10.1097/mcg.0000000000000350

Scherf, K. S., Behrmann, M., \& Dahl, R. E. (2012). Facing changes and changing faces in adolescence: a new model for investigating adolescent-specific interactions between pubertal, brain and behavioral development. Dev Cogn Neurosci, 2(2), 199-219. doi:10.1016/j.den.2011.07.016

Setayeshgar, S., Ekwaru, J. P., Maximova, K., Majumdar, S. R., Storey, K. E., McGavock, J., \& Veugelers, P. J. (2017). Dietary intake and prospective changes in cardiometabolic risk factors in children and youth. Appl Physiol Nutr Metab, 42(1), 39-45.

Singh, G., Kogan, M., Van Dyck, P., \& Siahpush, M. (2008). Racial/ethnic, socioeconomic, and behavioral determinants of childhood and adolescent obesity in the United States: analyzing independent and joint associations. Annals of Epidemiology, 18(9), 682-695. 
Slavin, J. L. (2008). Position of the American Dietetic Association: health implications of dietary fiber. J Am Diet Assoc, 108(10), 1716-1731.

Slavin, J. L. (2013). Fiber and prebiotics: mechanisms and health benefits. Nutrients, 5(4), 1417-1435. doi:10.3390/nu5041417

Solomons, N. (2006). Present Knowledge in Nutrition. 9th ed. . Washington, DC: International Life Sciences Institute.

Somerville, M., Kessler, L. A., Wallace, S. P., \& Burns-Whitmore, B. (2012). The Effect of a Garden-Based Nutrition Education Program on the Consumption of Fruits and Vegetables by Hispanic Children. Californian Journal of Health Promotion, $10(\mathrm{~S} 1), 20-25$.

Spahn, J. M., Reeves, R. S., Keim, K. S., Laquatra, I., Kellogg, M., Jortberg, B., \& Clark, N. A. (2010). State of the evidence regarding behavior change theories and strategies in nutrition counseling to facilitate health and food behavior change. $J$ Am Diet Assoc, 110(6), 879-891. doi:10.1016/j.jada.2010.03.021

Steffen, L. M. (2006). Eat your fruit and vegetables. The Lancet, 367(9507), 278-279. doi:10.1016/s0140-6736(06)68046-x

Sylvetsky-Meni, A. C., Gillepsie, S. E., Hardy, T., \& Welsh, J. A. (2015). The impact of parents' categorization of their own weight and their child's weight on healthy lifestyle promoting beliefs and practices. J Obes, 2015, 307381. doi: $10.1155 / 2015 / 307381$

Tadayon Nabavi, R. (2012). Bandura's Social Learning Theory \& Social Cognitive Learning Theory.

Taylor, J. H., Xu, Y., Li, F., Shaw, M., Dziura, J., Caprio, S., . . Savoye, M. (2016). Psychosocial predictors and moderators of weight management programme outcomes in ethnically diverse obese youth. Pediatr Obes. doi:10.1111/ijpo.12165

Thompson, C. A., Habermann, T. M., Wang, A. H., Vierkant, R. A., Folsom, A. R., Ross, J. A., \& Cerhan, J. R. (2010). Antioxidant intake from fruits, vegetables and other sources and risk of non-Hodgkin's lymphoma: the Iowa Women's Health Study. Int J Cancer, 126(4), 992-1003. doi:10.1002/ijc.24830

Tool: Knowledge, attitude, and consumption behavior survey. (2010). Wisconsin farm to table study. Retrieved from https://www.cias.wisc.edu/foodservtools14/7.../knowledge-attitudes-consumption.

Trost, S., Sirad, J., Dowda, M., Pfeiffer, K., \& Pate, R. (2003). Physical activity in overweight and nonoverweight preschool children. International Journal of Obesity, 27, 834-839.

Troxell, H., Anderson, J., Auld, G., Marx, N., Harris, M., Reece, M., \& Allen, K. (2005). Omega-3 for baby and me: material development for a WIC intervention to increase DHA intake during pregnancy. Matern Child Health J, 9(2), 189-197. doi:10.1007/s10995-005-4908-0

Tuohy, K. M., Fava, F., \& Viola, R. (2014). 'The way to a man's heart is through his gut microbiota'--dietary pro- and prebiotics for the management of cardiovascular risk. Proc Nutr Soc, 73(2), 172-185. doi:10.1017/s0029665113003911

United Nations, D. o. E. a. S. A., \& Division., P. (2015). World population prospects: the 2015 revision, key findings and advance tables. ’. Working paper No. ESA/P/WP.

USDA. (2010). Choose my plate. Retrieved from https://www.choosemyplate.gov/ 
USDA. (2012a). Census of agriculture. Retrieved from JoAnneWhelanDissertationApril2018.docx

USDA. (2012b). Federal Register: Nutrition standards in the national school lunch and school breakfast programs. Retrieved from https://www.fns.usda.gov/schoolmeals/nutrition-standards-school-meals.

USDA. (2012c). USDA School Guidelines. .

USDA. (2014, July 10, 2017). USDA fruit and vegetable program. Retrieved from https://www.ers.usda.gov/topics/food-nutrition-assistance/child-nutritionprograms/usda-fruit-and-vegetable-program/

USDA. (2016a). Child and Adult Care Food Program: Meal Pattern Revisions Related to the Healthy, Hunger-Free Kids Act of 2010. Final rule. Fed Regist, 81(79), $24347-24383$.

USDA. (2016b). Vitamins and minerals. Retrieved from https://fnic.nal.usda.gov/foodcomposition/vitamins-and-minerals

USDA. (2017). Choose my plate. Retrieved from https://www.choosemyplate.gov/MyPlate

USDHHS. (2016). Healthy people 2020. Retrieved from http:// www.healthypeople.gov/2020/default.

van Jaarsveld, C. H., Fidler, J. A., Simon, A. E., \& Wardle, J. (2007). Persistent impact of pubertal timing on trends in smoking, food choice, activity, and stress in adolescence. Psychosom Med, 69(8), 798-806.

doi:10.1097/PSY.0b013e3181576106

van Meer, F., van der Laan, L. N., Viergever, M. A., Adan, R. A. H., \& Smeets, P. A. M. (2017). Considering healthiness promotes healthier choices but modulates medial prefrontal cortex differently in children compared with adults. Neuroimage, 159, 325-333. doi:10.1016/j.neuroimage.2017.08.007

Vlasin-Marty, K., Ritter-Gooder, P., \& Albrecht, J. A. (2016). Food Safety Knowledge, Attitudes, and Behaviors of Native American Families with Young Children: A Mixed Methods Study. J Racial Ethn Health Disparities, 3(4), 713-723. doi:10.1007/s40615-015-0190-z

Walker, K., \& Jackson, R. (2015). The health belief model and determinants of oral hygiene practices and beliefs in preteen children: a pilot study. Pediatr Dent, $37(1), 40-45$.

Wang, X., Ouyang, Y., Liu, J., Zhu, M., Zhao, G., Bao, W., \& Hu, F. B. (2014). Fruit and vegetable consumption and mortality from all causes, cardiovascular disease, and cancer: systematic review and dose-response meta-analysis of prospective cohort studies. Bmj, 349. doi:10.1136/bmj.g4490

Watson, K., Baranowski, T., Thompson, D., Jago, R., Baranowski, J., \& Klesges, L. M. (2006). Innovative application of a multidimensional item response model in assessing the influence of social desirability on the pseudo-relationship between self-efficacy and behavior. Health Educ Res, 21 Suppl 1, i85-97. doi: $10.1093 /$ her/cyl 137

Wells, J. C., \& Fewtrell, M. S. (2006). Measuring body composition. Arch Dis Child, 91(7), 612-617. doi:10.1136/adc.2005.085522 
Wijesinha-Bettoni, R., Orito, A., Lowik, M., McLean, C., \& Muehlhoff, E. (2013). Increasing fruit and vegetable consumption among schoolchildren: efforts in middle-income countries. Food Nutr Bull, 34(1), 75-94.

Zhang, Q., \& Wang, Y. (2008). Childhood obesity in minority populations in the United States. North American Journal of Medicine and Science, 1(1), 44-47.

Zwald, M., Elliott, L., Brownson, R. C., \& Skala, M. (2015). Evidence-Based Diabetes Prevention and Control Programs and Policies in Local Health Departments.

Diabetes Educ, 41(6), 755-762. doi:10.1177/0145721715601736 


\section{CURRICULUM VITAE}

JoAnne Louise Whelan

\section{EDUCATION:}

- DOCTORAL

- GRADUATE

- UNDERGRADUATE
PhD, Indiana University, Health Science 2018

MS, Indiana University, Clinical Nutrition 2004

BS, Marian University, Dietetics

1999

\section{APPOINTMENTS:}

ACADEMIC

- Indiana University

NON-ACADEMIC

- Renal Care Group

Clinical Dietitian,

2000-2001

- Indiana University Health

Clinical Dietitian

2001-2005

- Eli Lilly Contract Personnel Research Dietitian

2004-2005

- Indiana University Health Pediatric Dietitian

2007-present

\section{LICENSURE:}

- Registered Dietitian

2000-present

\section{PROFESSIONAL ORGANIZATION MEMBERSHIPS:}

- Academy of Nutrition and Dietetics

2000-present

- Indiana Dietetic Association

2000-present 


\section{PROFESSIONAL HONORS AND AWARDS:}

- John Sweany Memorial Spirit Award Recipient Marian University 1999

- Marian College Student Association Leadership Marian University 1999

- Marian College Senior Award in Dietetics Marian University 1999

- Indiana Dietetic Association Scholarship Award Indiana Dietetics 1999

- Lute Troutt Fellowship Award Indiana University 2003

- Dr. Karyl Rickard Pediatric Scholarship Award Indiana University 2015

RESEARCH/CREATIVE ACTIVITY:

- Poster presenter for: More Fruits and Vegetables for Elementary School Children, April 2013

- Presenter at the National Down Syndrome Conference, Indianapolis, IN Nutrition for Growth and Development, July 2014

- Whelan, J. \& Ernst, J. Poster presenter for the Indiana University School of Health and Rehabilitation Sciences Conference:

Evaluation of Fruit and Vegetable Intake at a Private School, November 2015

TEACHING:

TEACHING ASSIGNMENTS

- Guest Lecturer for Physician Assistant Program: Nutrition and Pregnancy, 2014

UNDERGRADUATE

N420 Lifecycle Nutrition Online Instructor Spring/Fall 55 students 